Florida International University FIU Digital Commons

$11-8-2007$

\title{
Numerical Investigation on the Heat Transfer Enhancement Using Micro/Nano Phase-Change Particulate Flow
}

Keqiang Xing

Florida International University, kxing002@fiu.edu

DOI: $10.25148 /$ etd.FI08081550

Follow this and additional works at: https://digitalcommons.fiu.edu/etd

\section{Recommended Citation}

Xing, Keqiang, "Numerical Investigation on the Heat Transfer Enhancement Using Micro/Nano Phase-Change Particulate Flow" (2007). FIU Electronic Theses and Dissertations. 28.

https://digitalcommons.fiu.edu/etd/28

This work is brought to you for free and open access by the University Graduate School at FIU Digital Commons. It has been accepted for inclusion in FIU Electronic Theses and Dissertations by an authorized administrator of FIU Digital Commons. For more information, please contact dcc@fiu.edu. 


\title{
FLORIDA INTERNATIONAL UNIVERSITY
}

Miami, Florida

\section{NUMERICAL INVESTIGATION ON THE HEAT TRANSFER ENHANCEMENT USING MICRO/NANO PHASE-CHANGE PARTICULATE FLOW}

\author{
A dissertation submitted in partial fulfillment of the \\ requirements for the degree of \\ DOCTOR OF PHILOSOPHY \\ in \\ MECHANICAL ENGINEERING \\ by
}

Keqiang Xing

2007 
To: Interim Dean Amir Mirmiran

College of Engineering and Computing

This dissertation, written by Keqiang Xing, and entitled Numerical Investigation on the Heat Transfer Enhancement Using Micro/Nano Phase-Change Particulate Flow, having been approved in respect to style and intellectual content, is referred to you for judgment.

We have read this dissertation and recommend that it be approved.

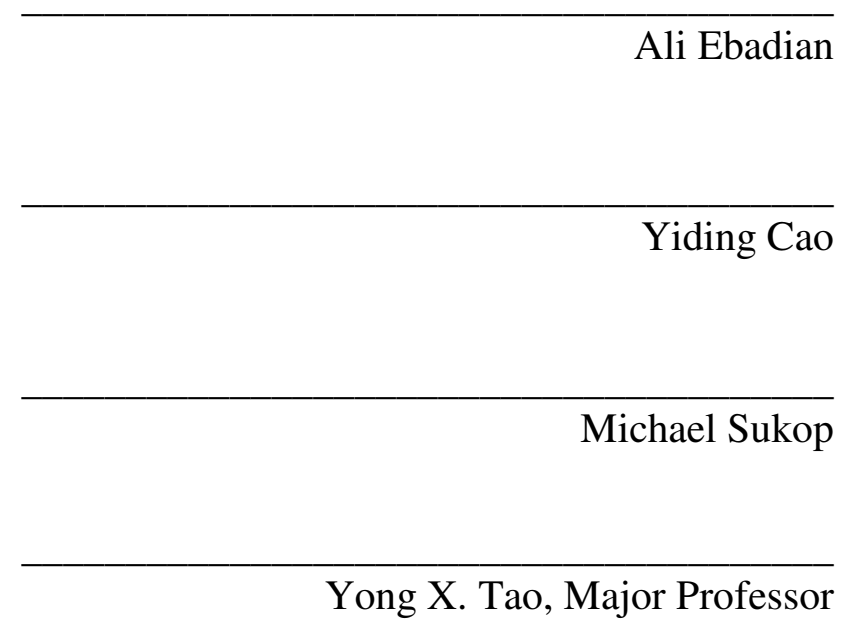

Date of Defense: November 8, 2007

The dissertation of Keqiang Xing is approved.

Interim Dean Amir Mirmiran
College of Engineering and Computing

Florida International University, 2007 


\section{DEDICATION}

I dedicate this dissertation to my parents and my wife, for their never-ending support and encouragement. 


\section{ACKNOWLEDGMENTS}

During this work, I was in contact with lots of people who helped me in many ways.

First of all, I would like to thank my advisor Dr. Yong X. Tao for his support and guidance throughout these years. He has been a true mentor. This work can not be done without numerous inspiring discussions with him. I thank Dr. Yingli Hao for building the foundation for this work, and helpful discussions about the numerical model. I thank Dr. Michael Sukop for introducing me to the lattice Boltzmann simulation, and for having patiently read and proposed corrections on the draft. I also would like to thank my other dissertation committee members, Dr. Ali Ebadian and Dr. Yiding Cao, for their helpful discussion and advice, and willingness to participate in this process.

Among my friends and fellow students, I thank Dr. Peng Yuan, Dr. Haibo Huang, and Mr. Quan Liao for helping me to understand the lattice Boltzmann method. I also thank Xiaohua Li, Ming Feng, Chunmiao Ye, Wenzhong Wu, Xudong Chen, Ryan Moreno, Jose Iragorry, Prasanna Maloji, etc., for their friendship and help in so many ways.

Finally, I would like to acknowledge the support from FIU's Presidential Fellowship. 


\title{
ABSTRACT OF THE DISSERTATION \\ NUMERICAL INVESTIGATION ON THE HEAT TRANSFER ENHANCEMENT USING MICRO/NANO PHASE-CHANGE PARTICULATE FLOW
}

\author{
by \\ Keqiang Xing \\ Florida International University, 2007 \\ Miami, Florida \\ Professor Yong X. Tao, Major Professor
}

The introduction of phase change material fluid and nanofluid in micro-channel heat sink design can significantly increase the cooling capacity of the heat sink because of the unique features of these two kinds of fluids. To better assist the design of a high performance micro-channel heat sink using phase change fluid and nanofluid, the heat transfer enhancement mechanism behind the flow with such fluids must be completely understood.

A detailed parametric study is conducted to further investigate the heat transfer enhancement of the phase change material particle suspension flow, by using the twophase non-thermal-equilibrium model developed by Hao and Tao (2004). The parametric study is conducted under normal conditions with Reynolds numbers of $\operatorname{Re}=90-600$ and phase change material particle concentrations of $\varepsilon_{p} \leq 0.25$, as well as extreme conditions of very low Reynolds numbers $(\operatorname{Re}<50)$ and high phase change material particle concentration $\left(\varepsilon_{p}=50 \%-70 \%\right)$ slurry flow. By using the two newly-defined parameters, named effectiveness factor $\varepsilon_{\text {eff }}$ and performance index $P I$, respectively, it is found that 
there exists an optimal relation between the channel design parameters $L$ and $D$, particle volume fraction $\varepsilon_{p}$, Reynolds number Re, and the wall heat flux $q_{w}$. The influence of the particle volume fraction $\varepsilon_{p}$, particle size $d_{p}$, and the particle viscosity $\mu_{p}$, to the phase change material suspension flow, are investigated and discussed. The model was validated by available experimental data. The conclusions will assist designers in making their decisions that relate to the design or selection of a micro-pump suitable for micro or mini scale heat transfer devices.

To understand the heat transfer enhancement mechanism of the nanofluid flow from the particle level, the lattice Boltzmann method is used because of its mesoscopic feature and its many numerical advantages. By using a two-component lattice Boltzmann model, the heat transfer enhancement of the nanofluid is analyzed, through incorporating the different forces acting on the nanoparticles to the two-component lattice Boltzmann model. It is found that the nanofluid has better heat transfer enhancement at low Reynolds numbers, and the Brownian motion effect of the nanoparticles will be weakened by the increase of flow speed. 


\section{TABLE OF CONTENTS}

CHAPTER

PAGE

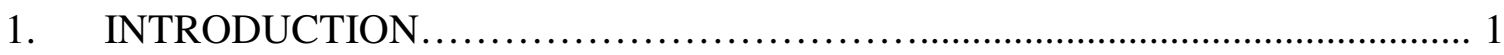

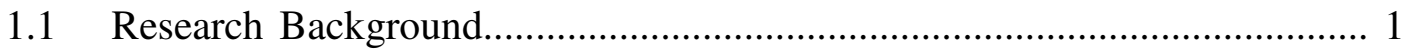

$1.2 \quad$ PCM Particle Suspension Flow.................................................................. 3

1.3 The Lattice Boltzmann Method.................................................................. 7

1.4 Objectives and Significance of the Study................................................... 9

2. PARAMETRIC STUDY WITH THE TRADITIONAL

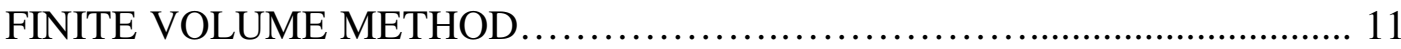

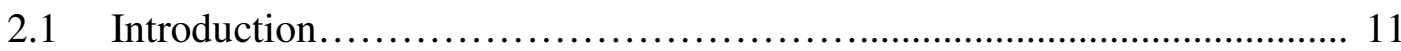

2.2 The Two-Phase Non-Thermal-Equilibrium Based Model............................ 12

2.3 Performance Evaluation of Liquid Flow with PCM in Micro-channels.... 19

2.3.1 Fluid-particle suspension flow............................... 19

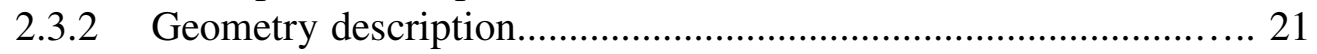

2.3.3 Boundary Conditions........................................... 22

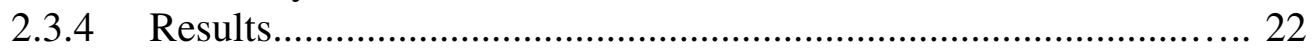

2.3.5 Conclusions................................................................................. 41

2.4 Low Reynolds Number Limit of Heat Transfer Enhancement with PCM Slurry Flow in Micro-channels..................................................... 43

2.4.1 Experiments.............................................. 44

2.4.2 Results....................................................... 47

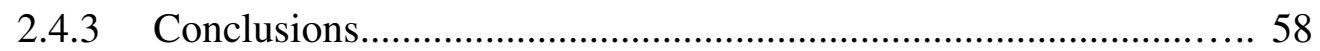

2.5 Slurry Viscosity Study and Its Influence on Heat Transfer Enhancement Effect of PCM Slurry Flow................................................... 60

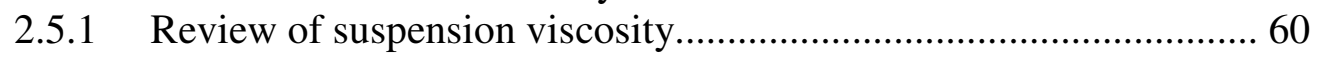

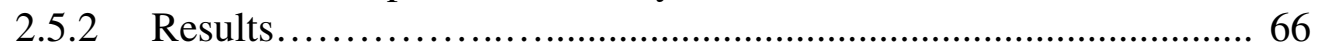

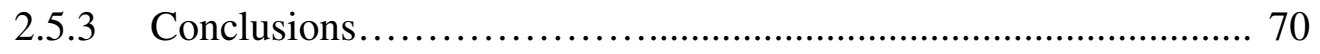

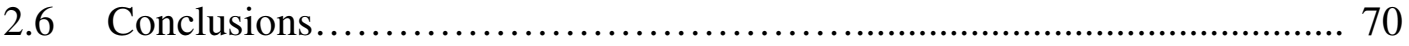

3. THE LATTICE BOLTZMANN METHOD_....................................................... 72

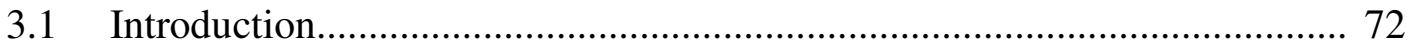

3.2 From Lattice Gas Automata to Lattice Boltzmann Method................... 72

3.3 From the Continuum Boltzmann Equation to Lattice Boltzmann Equation......................................... 75

3.4 Formulation of the LBM.......................................... 76

3.5 Boundary Conditions................................................. 79

3.5.1 Bounce-back boundary condition.................................................. 79

3.5.2 Periodic boundary condition........................................................ 80

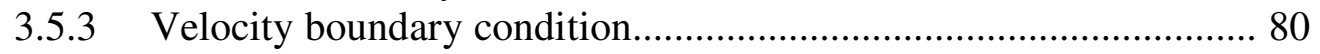

3.5.4 Pressure boundary condition......................................................... 82

3.6 Thermal Lattice Boltzmann Model..................................... 83

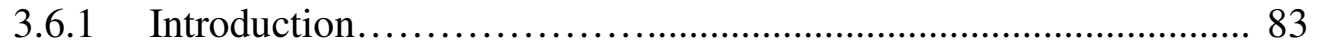


3.6.2 Formulation of the passive-scalar-based .TLBM............... 84

3.6.3 Boundary conditions............................................................. 85

3.7 Multicomponent Lattice Boltzmann Model............................. 87

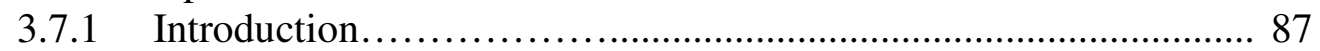

3.7.2 Formulation of the SC multicomponent LBM model............... 88

3.8 Benchmark Tests.......................................... 91

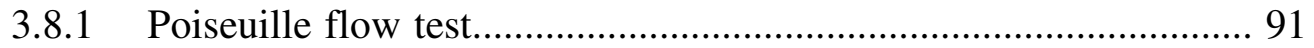

3.8.2 Rayleigh-Bénard convection.................................................. 92

3.8.3 Flow and heat transfer in a symmetric bifurcation channel test...... 94

3.9 Conclusions................................................. 107

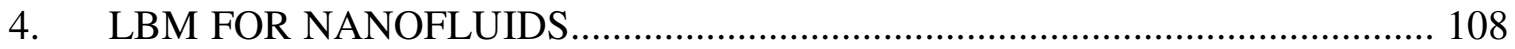

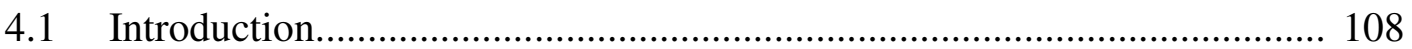

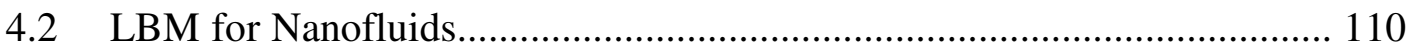

4.3 TLBM for Nanofluids.................................................................... 111

4.4 Force Evaluation of LBM for Nanofluids............................ 112

4.4.1 The buoyancy and gravitational force............................ 113

4.4.2 The drag force............................................... 113

4.4.3 The Brownian force............................................................... 114

4.4.4 The interaction potential.................................. 115

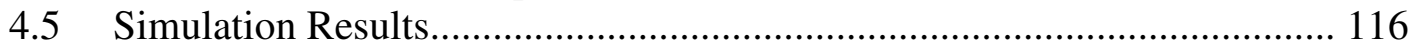

4.5.1 Problem description......................................... 116

4.5.2 Simulation results............................................ 117

4.6 Parametric Study of the Particle Cluster Size Effect......................... 123

4.7 Numerical Validation for Nanofluid LBM.......................... 126

4.7.1 Mass conservation.......................................... 126

4.7.2 Energy conservation....................................... 126

4.7.3 Grid independence....................................... 127

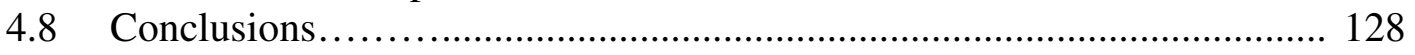

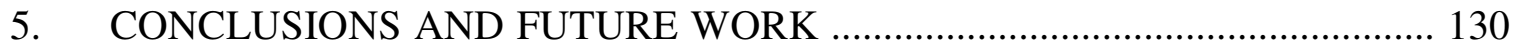

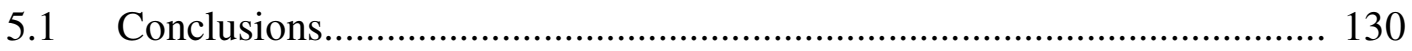

5.1.1 Conclusions from the parametric study by using

the traditional finite volume approach........................................ 130

5.1.2 Conclusions from the nanofluid simulation by LBM approach.... 132

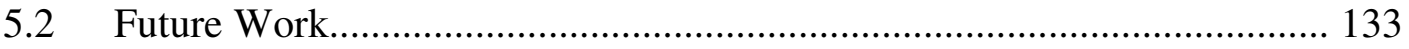

5.2.1 Review of the LBM simulation of solid-liquid

phase-change phenomena.................................. 133

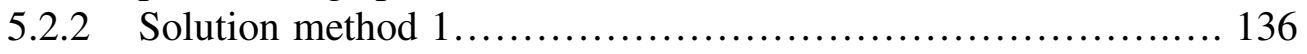

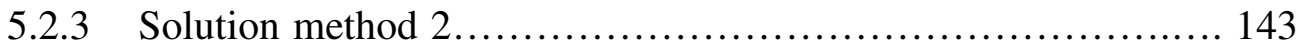

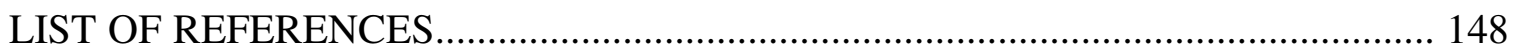

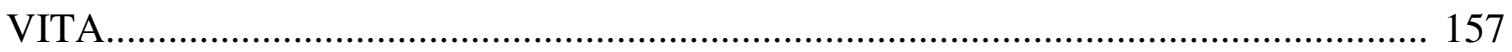




\section{LIST OF TABLES}

TABLE

PAGE

2.1 Thermophysical properties of octadecane PCM suspension flow with shell materials

2.2 Thermophysical properties of eicosane PCM suspension flow....

2.3 Thermophysical properties of octadecane PCM slurry without shell materials... 44

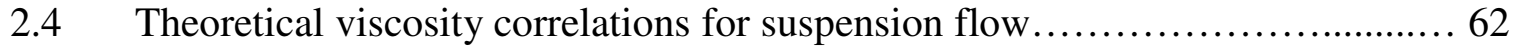

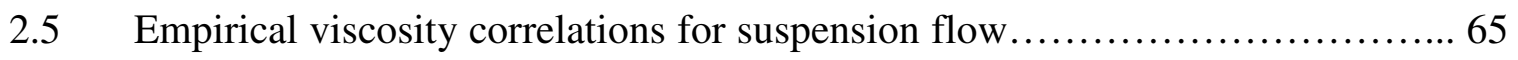

4.1 Lattice unit conversion rules......................................... 117 


\section{LIST OF FIGURES}

FIGURE

PAGE

2.1 Schematic diagram of fluid and PCM particle suspension

flowing through a heated tube. 13

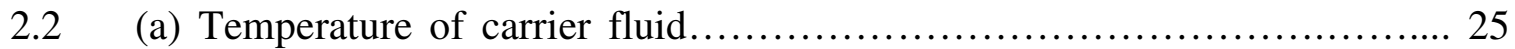

(b) Temperature of PCM particle.................................... 25

(c) Liquid mass fraction inside of PCM particle.......................... 25

2.3 Effectiveness factor and performance of slurry at different Reynolds numbers:

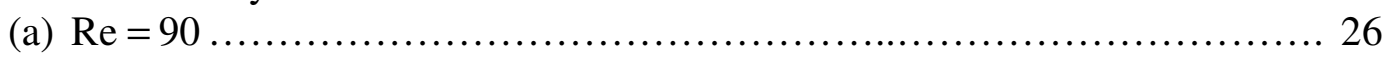

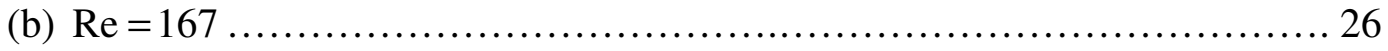

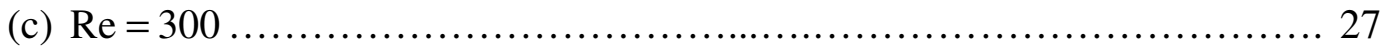

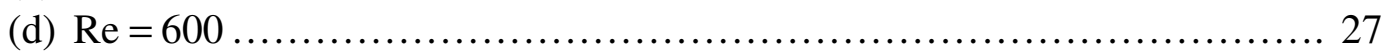

2.4 Maximum $\varepsilon_{\text {eff }}$ and corresponding $q_{w}\left(\mathrm{~W} / \mathrm{cm}^{2}\right)$ as a function of Re ............ 28

2.5 $Q / P$ ratios of slurry and pure water at different Reynolds numbers:

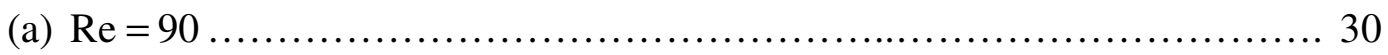

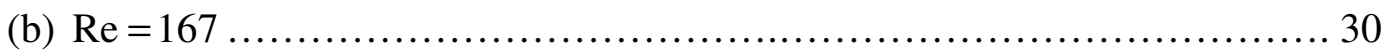

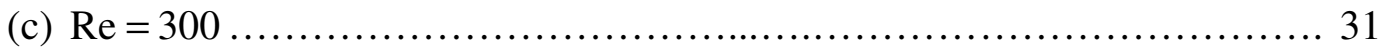

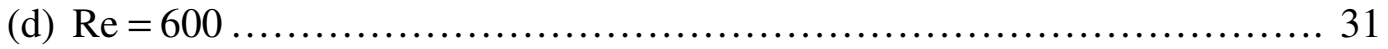

2.6 Local heat transfer coefficient along the channel at different Re with $q_{w}=50 \mathrm{~W} / \mathrm{cm}^{2}$ :

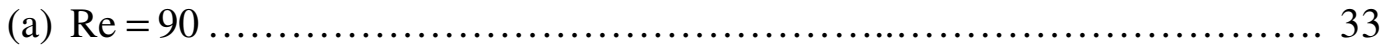

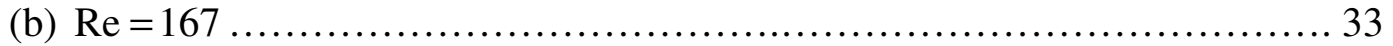

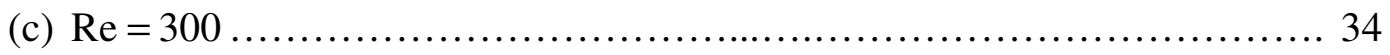

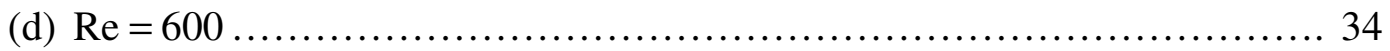

2.7 Distribution of local heat transfer coefficients where $\varepsilon_{\text {eff } \text {, max }}$ and $q_{w, \text { max }}$ occur at a given Re.....

2.8 Effect of particle volume fraction on $\varepsilon_{\text {eff }}$ with $\mathrm{Re}=300, q_{w}=30 \mathrm{~W} / \mathrm{cm}^{2} \ldots \ldots . .35$

2.9 Comparison of simulation results with the experimental data under the following conditions: $\varepsilon_{p}=0.1, \operatorname{Re}=200, d_{p}=100 \mu \mathrm{m}$ : 38

(a) Ste $=2.0$

(b) Ste $=3.0$ 
2.10 Liquid mass fraction inside of PCM particle with $\varepsilon_{p}=0.1, \operatorname{Re}=200, d_{p}=100 \mu \mathrm{m}$, Ste $=2.0$ :
(a) $T_{\text {in }}=309.85 \mathrm{~K}$
(b) $T_{\text {in }}=309.9 \mathrm{~K}$

2.11 Schematic diagram of the experimental setup:
(a) Entire system
(b) Cross-sectional view of the test section

2.12 Effectiveness factor and performance index of PCM suspension flow:
(a) $\varepsilon_{p}=0.5, \mathrm{Re}=10$ 48
(b) $\varepsilon_{p}=0.7, \mathrm{Re}=10$ 48
(c) $\varepsilon_{p}=0.5, \mathrm{Re}=30$ 49
(d) $\varepsilon_{p}=0.7, \mathrm{Re}=30$. 49
(e) $\varepsilon_{p}=0.5, \operatorname{Re}=40$ 50
(f) $\varepsilon_{p}=0.7, \operatorname{Re}=40$ 50

2.13 Maximum $\varepsilon_{e f f}$ as a function of Re at different particle concentrations 51

2.14 Optimal $q_{w}$ as a function of Re at different particle concentrations. 51

2.15 Maximum $\varepsilon_{e f f}$ as a function of $\varepsilon_{p}$ at different Reynolds numbers.

2.16 Comparison between the simulation results and the correlation:

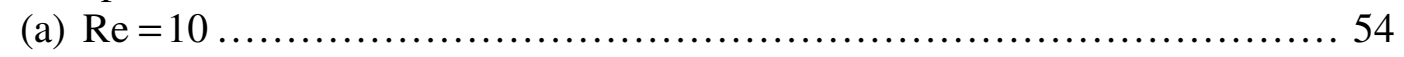

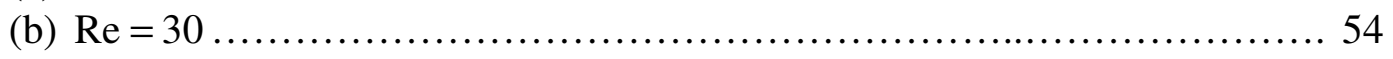

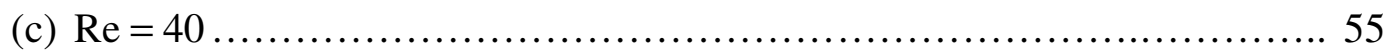

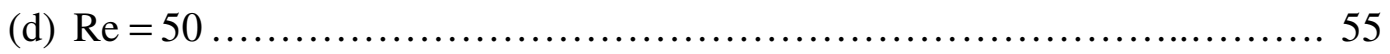

2.17 Effect of particle volume fraction on $\varepsilon_{\text {eff }}$

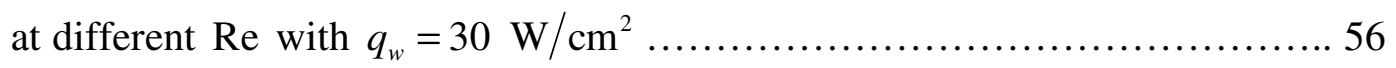

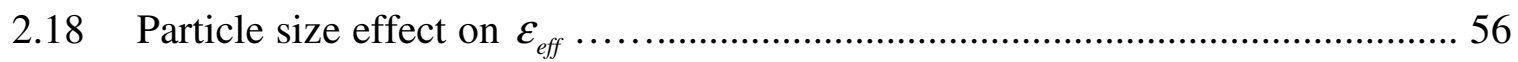

2.19 Comparison between the experiment and simulation results............................... 58

2.20 Plot of correlations of bulk viscosity for suspension flow................................... 67

2.21 Influence of particle viscosity to the heat transfer enhancement effect of PCM suspension flow 
3.1 Sketches of the most common lattices............................................................ 77

3.2 Illustration of fullway and halfway bounce-back boundary conditions:......... 80

(a) fullway

(b) halfway

3.3 Schematic plot of distribution function of D2Q9 model in a channel flow

3.4 Velocity profile for Poiseuille flow from LBM simulation and analytical solution

3.5 Rayleigh-Bénard convection at $R a=5000$ and $\operatorname{Pr}=1$ :

(a) Velocity vector

(b) Isotherms

3.6 The geometry of the simulated symmetric bifurcation.

3.7 Simulation results for $\theta=45^{\circ}$ and $q=1$ at different Reynolds numbers:

(a) Velocity magnitude................................................ 97

(b) Temperature field................................................... 98

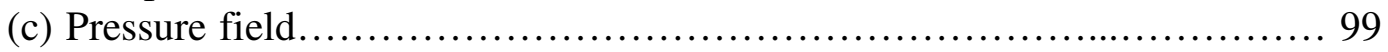

3.8 Temperature fields for different heat fluxes at $\theta=45^{\circ}$ and $\operatorname{Re}=6 \ldots \ldots \ldots \ldots . .100$

3.9 Simulation results for $\operatorname{Re}=6$ and $q=1$ at different bifurcation angles:

(a) Velocity magnitude............................................... 101

(b) Temperature field................................................. 102

(c) Pressure field........................................................ 103

3.10 Comparison between FLUENT and our simulation results at $\theta=45^{\circ}, \operatorname{Re}=12$ and $q=1$ :

(a) Velocity magnitude................................................. 104

(b) Temperature field.................................................... 105

(c) Pressure field..................................................... 106

4.1 Temperature filed of nanofluid at different $\varepsilon_{p}$ with $\operatorname{Re}=5, q=1 \ldots \ldots \ldots \ldots \ldots .119$

4.2 Temperature field of nanofluid at different Re with $\varepsilon_{p}=3 \%, q=1 \ldots \ldots \ldots \ldots .120$

4.3 Average heat transfer coefficient of nanofluid along the channel at different $\varepsilon_{p}$ with $\operatorname{Re}=5, q=1$. 
4.4 Average heat transfer coefficient of nanofluid along the channel at different Re with $\varepsilon_{p}=3 \%, q=1$

4.5 Temperature field of nanofluid along the channel at different $q$ with $\varepsilon_{p}=3 \%, \operatorname{Re}=5$

4.6 Average heat transfer coefficient of nanofluid along the channel at different $h_{\sigma \bar{\sigma}}$ with $\varepsilon_{p}=3 \%, \operatorname{Re}=5, q=1$

4.7 Particle cluster size effect at $\varepsilon_{p}=1 \%, \operatorname{Re}=5, q=1$

4.8 Temperature field at two different meshes................................ 128

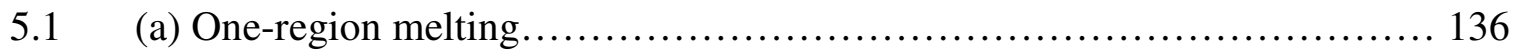

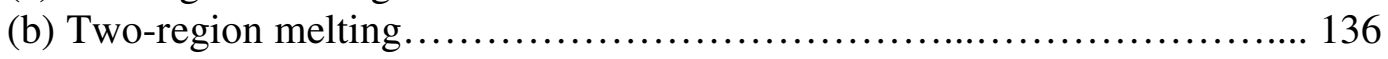

5.2 Dimensionless temperature distribution in the PCM 139 


\section{LIST OF SYMBOLS}

$c$

$c_{s}$

$c_{p}$

D

$d_{p}$

$\mathbf{e}_{\mathrm{i}}$

$f_{i}$

$f_{i}^{e q}$

$f_{p, l}$

$g$

$g_{i}$

$g_{i}^{e q}$

$G r$

$h$

$k$

$L$

$\mathrm{Nu}$

$p$

$P$

PI

$q$ lattice speed

lattice speed of sound

specific heat at constant pressure

tube diameter

particle diameter

lattice velocity vector

friction factor in the definition of $P I$

particle distribution function

equilibrium particle distribution function

liquid mass fraction inside PCM particles

gravitational acceleration

particle distribution function for temperature field

equilibrium particle distribution function for temperature field

Grashof number

heat transfer coefficient

thermal conductivity; constant in slurry viscosity correlations

tube length; latent heat

Nusselt number

pressure

pumping power

performance index

heat flux 


$\begin{array}{ll}Q & \text { heat transfer rate } \\ R e & \text { Reynolds number } \\ T & \text { temperature } \\ t & \text { time } \\ \mathbf{u}, u_{y} & \text { fluid velocity } \\ V & \text { inlet velocity } \\ w_{i} & \text { weighting factor } \\ \mathbf{x}, \mathbf{x} & \text { particle position }\end{array}$

\section{$\underline{\text { Greek Symbols }}$}

$\begin{array}{ll}\alpha & \text { thermal diffusivity } \\ \beta & \text { thermal expansion coefficient } \\ \delta t & \text { lattice time step } \\ \delta x & \text { lattice spacing } \\ \varepsilon & \text { volume fraction in the suspension } \\ \varepsilon_{e f f} & \text { effectiveness factor } \\ \varepsilon_{m} & \text { maximum packing fraction } \\ \theta & \text { bifurcation angle; dimensionless temperature } \\ \mu & \text { dynamic viscosity } \\ v & \text { kinematic viscosity } \\ \rho & \text { density } \\ \tau & \text { relaxation time; dimensionless time }\end{array}$




$\begin{array}{ll}\tau_{T} & \text { relaxation time for temperature field } \\ \psi & \text { source term }\end{array}$

\section{$\underline{\text { Subscripts }}$}

$\begin{array}{ll}\text { ave } & \text { average } \\ b & \text { bulk } \\ f & \text { carrier fluid } \\ \text { in } & \text { inlet } \\ l & \text { liquid phase } \\ l u & \text { lattice unit } \\ m & \text { melting } \\ \text { max } & \text { maximum } \\ \text { opt } & \text { optimal } \\ \text { out } & \text { exit } \\ p & \text { PCM particle } \\ r & \text { relative } \\ s & \text { solid phase } \\ w & \text { wall }\end{array}$

\section{$\underline{\text { Abbreviations }}$}

BC boundary condition

BGK Bhatnagar-Gross-Krook

CFD computational fluid dynamics 


$\begin{array}{ll}\text { LB } & \text { lattice Boltzmann } \\ \text { LBE } & \text { lattice Boltzmann equation } \\ \text { LBGK } & \text { lattice BGK } \\ \text { LBM } & \text { lattice Boltzmann method } \\ \text { LGA } & \text { lattice gas automata } \\ \text { MCPCM } & \text { microencapsulated phase change material } \\ \text { NPCM } & \text { nano-size phase change material } \\ \text { N-S } & \text { Navier-Stokes } \\ \text { PCM } & \text { phase change material } \\ \text { SC } & \text { Shan \& Chen } \\ \text { TLBM } & \text { thermal lattice Boltzmann method }\end{array}$




\section{CHAPTER 1}

\section{INTRODUCTION}

\subsection{Research Background}

Since the introduction of the concept of micro-channel heat sinks for electronic cooling by Tuckerman and Pease in 1981, there has been explosive growth in the field of ultra-compact heat sinks and integrated cooling devices, especially for high-flux applications. The cooling capacity of microelectronics can directly influence its switching speed. A cooling capacity in the order of $10^{5} \mathrm{~W} / \mathrm{cm}^{3}$ is required, in order to reach an order of up to $3.8 \times 10^{24}$ bits per second per $\mathrm{cm}^{2}$ switching speed for next generation computing devices (Drexler, 1992; Frank and Knight, 1998). This requirement is almost impossible for the capability of spreader-air-cooling technique (Ortega, 2002), and also outreached by current liquid cooling systems, including micro-channel heat sinks. Therefore, active liquid cooling needs to advance to achieve the further heat enhancement capability, which is especially important for 3-dimensional electronic packaging (Gromoll, 1994; Hao and Tao, 2004).

One of such enhancement techniques is to utilize phase change material (PCM) fluids. The concept of a carrier fluid for encapsulated particles of phase change materials was first introduced by Mehalick and Tweedie in 1975. After that, a lot of research work has been conducted on the PCM suspension flow because of its many special features such as high energy storage density, low pumping power requirements, and high heat transfer rates between the wall and the suspension. Tao et al. (2003) proposed a new design of a scalable heat sink containing 3-D micro/nano network, utilizing liquid mixed with nano-size phase change materials (NPCMs) and having a high surface-to-volume 
ratio geometry. The conceptual design is capable of reaching cooling capacity of $10^{5} \mathrm{~W} / \mathrm{cm}^{3}$ by using encapsulated NPCM particles, which would result in an order of magnitude higher cooling capacity than typical micro-channel heat sink of the same volume and same pumping power.

To better assist the design of a high performance micro-channel heat sink using PCM fluid, the mechanisms behind flow using PCM heat transfer fluid must be completely understood. For this purpose, Hao and Tao (2004) developed a numerical model to unveil the heat transfer characteristics of PCM particles suspension in energy storage and thermal control systems. By considering the suspended solid particles as a continuum phase, the model is based on the continuum theory for the system of solid particles suspended in a Newtonian gas or liquid in a laminar flow. Although it has been demonstrated (Hao and Tao, 2004) that the model captures the essential physical phenomena such as particle-depletion boundary effects and non-equilibrium temperature distribution between two phases, the data necessary for design is still missing. Therefore, a detailed parametric study which can give direct guidance to the designer is in great need.

Another enhancement technique is to utilize nanofluid. Consisting of nanometersized particles suspended in base fluid, nanofluid has been proven to be effective in enhancing the performance of energy transport systems (Eastman et al., 2004). Because of its unique features such as very high thermal conductivity at very low nanoparticle concentration and difficult to settle down, nanofluid is considered to be very promising for nanotechnology-based heat transfer applications To use nanofluid in a high performance micro-channel sink, the heat transfer enhancement mechanism of nanofluid need to be investigated. Several theory models have been developed to explain the heat 
transfer mechanism of nanofluid (Xuan and Roetzel, 2000; Khabafer et al., 2003), based on the traditional heat transfer theory. Xuan and Yao (2005) proposed a lattice Boltzmann model for nanofluid through incorporating different forces acting on the nanoparticles. Because of the mesoscopic feature of the lattice Boltzmann method, this model can reveal the heat transfer mechanism of nanofluid from the particle level. Therefore, it is necessary to investigate the heat transfer mechanism of nanofluid by using the lattice Boltamann method.

\subsection{PCM Particle Suspension Flow}

Many thermal energy systems have long sections of piping to convey heat transfer fluids between the heat exchangers for source and sink. In such conventional systems, thermal energy is transferred by the sensible heat of a single-phase working fluid, being proportional to the source-sink temperature difference. Because the systems are often operated with small temperature differences, the single-phase fluid must be pumped at a high volume flow rate. As a result, the system consumes a large amount of pumping power. The increase in the thermal capacity of heat transfer fluid is an important problem and often a growing concern to engineers.

The use of PCM particles suspended in a single-phase working fluid would provide additional thermal capacity from the latent heat associated with the solid-liquid phase change. Several methods for generating PCM particles have been investigated for various thermal energy applications. For district cooling systems, ice-water slurry has been developed and is implemented in practice. Cleary et al. (1990) found that ice slurry of $25 \%$ in particle volume concentration had a thermal capacity that was 2 to 4 times higher than that of chilled water. Choi et al (1994) developed a system that generated solid 
hexadecane particles of $0.1 \mathrm{~mm}$ in size, using an emulsifier, and they studied the heat transfer characteristics of the hexadecane-water slurry. However, because such particles of non-encapsulated PCM are slightly sticky and can stick together to form large lumps, clogging often occurs in a piping system. In addition, such systems require relatively large equipment for generating the particles, which results in an associated high capital investment for the system. Therefore, the use of microencapsulated PCM (MCPCM) slurry as a heat transfer fluid was proposed because the MCPCM particles are always separated from the carrier fluid.

The use of MCPCM suspensions as heat transfer fluids had been suggested over two decades ago. These suspensions benefit from a number of special features including: (a) a high energy storage density due to the absorption of latent heat during the phase change process; (b) relatively low variations in operating temperatures of systems using such fluids due to energy absorption at approximately constant temperature; (c) the possibility of using the same medium for both energy transport and storage, thereby reducing losses during the heat exchange process; (d) lower pumping power requirements due to the increased heat capacity; (e) high heat transfer rates to the phase change material due to large surface area to volume ratio; (f) the enhanced thermal conductivity of suspensions leading to increased heat transfer to the suspension; and $(\mathrm{g})$ the reduction/elimination of incongruent melting and phase separation.

Previous studies on convective heat transfer of MCPCM suspensions, performed both experimentally and theoretically, showed that, compared with single-phase fluids, MCPCM slurry can enhance convective heat transfer performance resulting in appreciable reduction in mass flow rate, wall temperature and pumping power. Mehalick 
and Tweedie (1975) first introduced the concept of a carrier fluid for encapsulated PCM particles, and Bahrami (1982) later studied the feasibility of their use at high temperature and heat fluxes. Hart and Thornton (1982) reported a two-fold increase in the effective specific heat of a suspension of $30 \%$ wax capsules in oil tested in a prototype solar collection pump at a temperature range of $100-120{ }^{\circ} \mathrm{C}$. McMahon et al. (1982) carried out thermal performance tests of slurries of $n$-heptadecane and $n$-octadecane in waterethylene glycol as cooling fluids for protective garments, and reported an effective specific heat increase of $20 \%$. Kasza and Chen (1982) estimated that the heat transfer coefficient could be increased by up to three times by the use of microencapsulated phase change suspensions. Colvin et al. (1989) reported specific heat increases up to 5 times and heat transfer coefficient increases of up to 2.8 times in flows of microencapsulated phase change material suspensions. Colvin and Mulligan (1990) obtained a patent for closed loop cooling systems with the heat source and sink physically close to each other and with both temperatures near the melting point of the phase change material. Under these circumstances, significant heat transfer enhancements and reductions in pumping powers can be obtained. Goel et al. (1994) studied the laminar forced convective heat transfer performance of a MCPCM-water suspension flowing in a circular tube with various MCPCM particle volume concentrations from $0-20 \%$. In their study, they analyzed the effects of Stefan number, volume concentration and particle size on the heat transfer performance by conducting comparative experiments at the same Reynolds number. Compared with water, they observed a significant reduction in wall temperature rise of up to 50\% for MCPCM suspensions. Yamagishi et al. (1999) experimentally investigated the hydrodynamics and heat transfer characteristics of MCPCM slurry. They 
found that increases in particle volume fractions caused the slurry flow structure to change from turbulent to laminar, and the pressure-drop reduction of the slurry flow relative to a single-phase water flow was under the same flow rate conditions. They claimed that the MCPCM slurry heat transfer effect was influenced by the MCPCM fraction, the degree of turbulence, and the heating rate at the tube wall. Recently, Inaba et al. (2004) examined the laminar and turbulent heat transfer characteristics of a $20 \%$ mass fraction MCPCM suspension with particles of different sizes flowing in a circular tube with constant heat flux at the wall. They revealed that the average heat transfer coefficients of the MCPCM suspension flows were 2-2.8 times larger than those of pure water flow under the same Reynolds number. In the turbulent flow region, the friction factor of the slurry was found to be lower than that of pure water due to the drag reducing effect of the particles.

Chen and Chen (1987) developed a model for heat transfer augmentation using phase change suspension flows over flat plates, and conducted experiments to test the validity of the model. They reported excellent agreement in their experimental and theoretical results which showed as much as threefold increase in the heat transfer coefficient. Charunyakorn et al. (1991) conducted a numerical simulation of MCPCM suspension flow in circular tubes at different boundary conditions for low temperature applications, by using a quasi-steady model for the phase change part. Their parametric study shows that the phase change material heat transfer in suspension flows is dependent on the bulk Stefan number and volumetric particle concentration. Their model also predicts augmentation of heat transfer coefficient by $1.5-3$ times, combined with a $40-60 \%$ reduction in temperature rise. Zhang and Faghri (1995) proposed a numerical 
solution for laminar forced convection heat transfer of a MCPCM suspension in a circular tube with constant heat flux. They solved the melting in the microcapsule by a temperature transforming model instead of a quasi-steady model, because the quasisteady model only considered the sensible heat of the MCPCM. By comparing with Goel et al. (1994)'s experimental results, they found good agreement between the numerical and the experimental results. They also found the quasi-steady model will exaggerate the effect of the MCPCM on the heat transfer of the suspension by comparing with Charunyakorn et al. (1991)'s simulation results. Hu and Zhang (2002) also conducted a numerical analysis for the forced convective heat transfer enhancement of MCPCM slurry in a circular tube with constant heat flux. By using an effective specific heat capacity model, they analyzed the influence of various factors and proposed a modification to the conventional Nusselt number correlations for convective heat transfer of internal flow.

While all the previous studies and experiments indicate promising applications of the MCPCM slurry as a heat transfer and storage medium, data necessary for design in scale-up applications are very incomplete. Therefore, it is important and necessary to find the optimum design conditions for different design needs, which will be given in detail in chapter 2 .

\subsection{The Lattice Boltzmann Method}

The numerical simulation of suspensions is traditionally handled by mesoscopic stochastic particle methods, such as Brownian-Stokesian dynamics, in which the effects of microscopic scales on the mesoscopic ones are represented via stochastic sources. The suspended particle-particle interactions take place via the intermediate of the surrounding 
fluid and give rise to long range forces which make the problem computationally very intensive. These long range forces result from the coherent superposition of short range interactions between the suspended macro-particles and the fluid molecules. However, a truly molecular treatment is ruled out by the huge scale separation between fluid molecules and the suspended particles. Hence, this problem is an appetizing opportunity for the mesoscopic lattice Boltzmann model.

As an independent numerical method for hydrodynamic simulations, the lattice Boltzmann method (LBM) originates from the microscopic understanding of physical phenomena, and pays attention to the interactions among particles at the molecular level. An important advantage of the LBM is that the mesoscopic physical interactions of the particles can be conveniently incorporated into the numerical model, thus it bridges the gap between the microscopic world and the macroscopic phenomenology, and the "the flow with suspended particles" has been described as a "must-use" class application of the LBM (Succi, 2001).

The LBM has been applied to a variety of flow field and heat transfer simulations, such as magnetic hydrodynamics, flow in dynamic geometries, turbulence and large eddy simulation, Bénard convection, flow in porous media, and global ocean circulation. The LBM has been particularly successful in the area of complex fluids including multiphase and multicomponent fluids, suspensions in fluid, viscoelastic flow, and chemical reaction flow. Therefore, the LBM can be used to investigate the flow and heat transfer characteristics of the PCM suspension flow. A detailed introduction to the LBM and some benchmark test results will be given in chapter 3 . 


\subsection{Objectives and Significance of the Study}

The primary objective of this study is to numerically investigate the heat transfer enhancement mechanism of the PCM particle suspension flow in a micro-channel, thus to provide design guidance for the design of a high performance micro-channel heat sink. The secondary objective is to use a mesoscopic numerical method called LBM to investigate the heat transfer enhancement mechanism of the nanofluid flow from the particle level.

Although there has been a lot of research work on the heat transfer effect of the PCM suspension flow, experimentally or numerically, data necessary for design is quite a few. The current research can give us a clear understanding of the complicated flow and heat transfer mechanisms of the PCM slurry flow. By using the two newly-defined parameters called effectiveness factor and performance index, the influence of particle concentration, particle size, inlet temperature, Reynolds number, and micro-channel geometry, on the heat transfer enhancement effect of PCM suspension flow, is clearly revealed. The optimum condition, which is a unique match of all the above-mentioned parameters, is found under normal conditions with Reynolds numbers of $\operatorname{Re}=90-600$ and PCM particle concentrations of $\varepsilon_{p} \leq 0.25$, as well as extreme conditions of very low Reynolds numbers $(\operatorname{Re}<50)$ and high phase change material particle concentration $\left(\varepsilon_{p}=50 \%-70 \%\right)$ slurry flow.

Based on the parametric investigation, this study, for the first time in this research area, generates a correlation between the maximum effectiveness factor, PCM particle 
concentration, and Reynolds number. This correlation can be reasonably used by designer in designing a PCM fluid micro-channel heat sink.

Compared to Hao and Tao (2004)'s work, this study is more thorough and more in detail. And also for the first time in this area, by conducting a detailed review to the available experimental and theoretical correlations for suspension viscosity, this study discusses the influence of the particle viscosity value to the heat transfer enhancement of the PCM suspension flow. Through comparison with experimental data, a more reasonable correlation for particle viscosity is proposed. It is also found that a particle viscosity which is lower than the water viscosity, gives a better approximation to the experimental data under investigation.

By using the mesoscopic lattice Boltzmann method, the heat transfer mechanism of the nanofluid is investigated through incorporating all kinds of forces acting on the nanoparticles. By introducing the cluster size concept, the current nanofluid model shows significant heat transfer enhancement compared to single-phase fluid. It is found that the nanofluid has better heat transfer enhancement at low Reynolds numbers, and the Brownian motion effect of the nanoparticles will be weakened by the increase of flow speed. 


\section{CHAPTER 2}

\section{PARAMETRIC STUDY}

\section{WITH THE TRADITIONAL FINITE VOLUME METHOD}

\subsection{Introduction}

The idea of designing a heat sink using PCM particle suspensions, called phase change heat sink, has been examined by different researchers. O'Connor and Weber (1997) measured the performance of a paraffin PCM heat sink. Through comparison with a solid aluminum heat sink, the phase change heat sink had much better temperature reduction. Pal and Joshi (1998) conducted a combined experimental and computational investigation of a transient thermal control of an avionics modules using PCM heat sink. Zheng and Wirtz (2000) used numerical models and experiments to optimize the performance of a phase change heat sink. Leland and Recktenwald (2003) numerically optimized the geometry of a phase change heat sink for extreme environments. They discussed the optimum design for a given combination of heat load, conductance to ambient, and phase change materials.

Tao et al. (2003) proposed a new design of a scalable, heat sink containing 3-D micro/nano network, utilizing liquid mixed with nano-size phase change materials and having a high surface-to-volume ratio geometry. To understand the mechanisms behind flow using PCM heat transfer fluid, Hao and Tao (2004) developed a numerical model to unveil the heat transfer characteristics of PCM particles suspension in energy storage and thermal control systems. The model is based on the continuum theory for the system of solid particles suspended in a Newtonian gas or liquid in a laminar flow. It has been demonstrated (Hao and Tao, 2004) that the model captures the essential physical 
phenomena such as particle-depletion boundary effects and non-equilibrium temperature distribution between two phases.

In this chapter, Hao and Tao's (2004) model will be used to conduct parametric study, for the purpose of further investigating the heat transfer enhancement mechanism of the PCM suspension flow. The parametric study includes finding the optimal heat transfer enhancement parameters, heat transfer enhancement effect under very low Reynolds number and high PCM concentration, and the influence of particle viscosity value to the heat transfer enhancement effect.

\subsection{The Two-Phase Non-Thermal-Equilibrium Based Model}

A schematic diagram describing a typical PCM particle suspension flow problem is shown in Fig. 2.1. The flow of carrier fluid with PCM particles at the temperature below the phase change temperature of PCM enters the heated tube. As heat transfer between the suspension and the heated wall occures, PCM particles absorb the thermal energy and melt while its temperature reaches the phase change temperature. The presence of PCM particles results in momentum and energy interaction between the particles and carrier fluid, collisions between the particles, and heat transfer among the particles, carrier fluid and the tube wall. Those combined interactions makes the hydrodynamic and heat transfer characteristics in the suspension flow very different from those in the pure fluid flow. For example, when phase change occurs, a fraction of the particles become liquid. Therefore, we would have the coexistence of carrier fluid, solid phase and liquid phase of PCM. They also move at different velocities and temperature. This requires a separate, detailed treatment of each phase (Hao and Tao, 2004). 


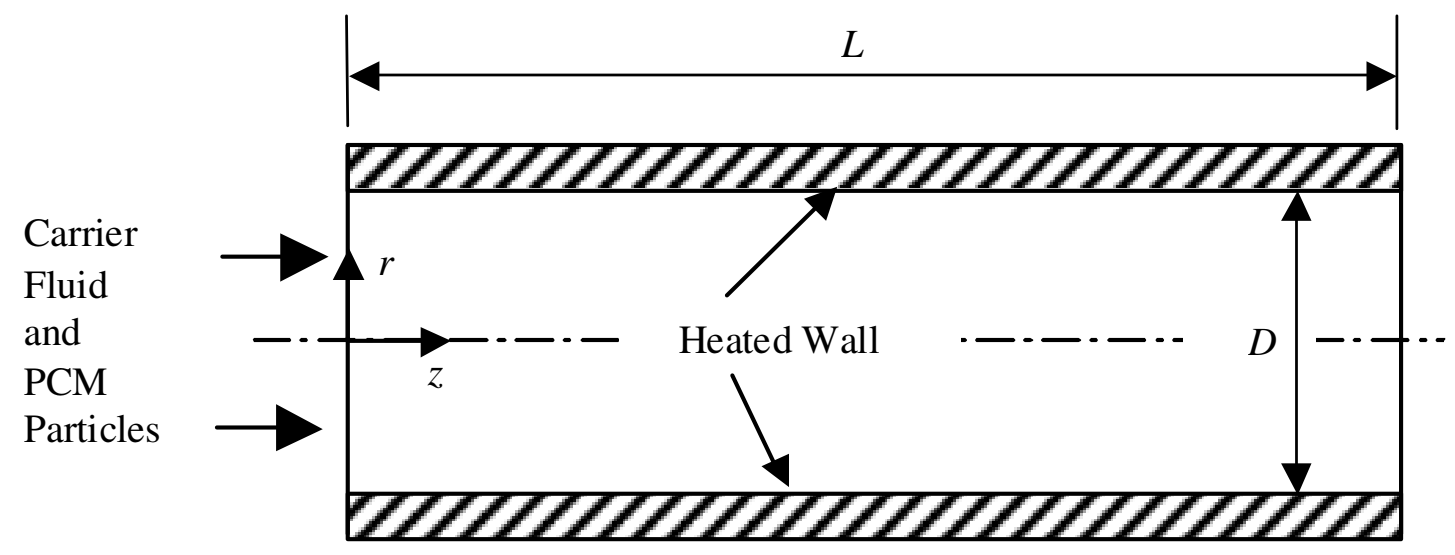

Figure 2.1 Schematic diagram of fluid and PCM particle suspension flowing through a heated tube. (Source: Hao and Tao, 2004)

The two-phase, non-thermal-equilibrium model with heat-mass transfer proposed by Hao and Tao (2004) is based on the theory of interacting continua. The continuum model for the suspension flow in a microchannel is based on the following observations: There are around 25 million molecules of air in a 1- $\mu \mathrm{m}$ cube at standard temperature and pressure. The same cube would contain around 34 billion molecules of water. Therefore, liquid flow is still a continuum medium even in micrometer-scale tube, while a gas flow through the same size tube might or might not be, depending on the gas Knudsen number. The average distance between molecules in the gaseous phase is one order of magnitude higher than the diameter of its molecules, while that for the liquid phase approaches the molecular diameter. As a result, most liquid flows are nearly incompressible. Therefore, we assume that the continuum model can be extended to the suspension flow in a microchannel. 
In the model, both liquid and solid phases are considered to be continuous and fully interpenetrating. Both phases are described in terms of separate conservation equations with appropriate interaction terms representing the coupling between the phases, including phase change. Fluid phase properties and the physical characteristics of the PCM particles, such as shape and size, are included in the continuum representation.

The volume-averaged equations of mass and momentum conservation for the laminar flow can be expressed as follows:

\section{Continuity equations:}

$$
\begin{aligned}
& \frac{\partial}{\partial t}\left(\varepsilon_{f} \rho_{f}\right)+\nabla \cdot\left(\varepsilon_{f} \rho_{f} \mathbf{v}_{f}\right)=0 \\
& \frac{\partial}{\partial t}\left(\varepsilon_{p} \rho_{p}\right)+\nabla \cdot\left(\varepsilon_{p} \rho_{p} \mathbf{v}_{p}\right)=0
\end{aligned}
$$

Momentum equations:

$$
\begin{aligned}
\frac{\partial}{\partial t}\left(\varepsilon_{f} \rho_{f} \mathbf{v}_{f}\right)+\nabla \cdot\left(\varepsilon_{f} \rho_{f} \mathbf{v}_{f} \mathbf{v}_{f}\right) & =-\varepsilon_{f} \nabla p+\nabla \cdot\left[\varepsilon_{f} \mu_{f}\left(\nabla \mathbf{v}_{f}+\nabla \mathbf{v}_{f}{ }^{T}\right)\right] \\
& +\varepsilon_{f} \rho_{f} \mathbf{g}-\beta\left(\mathbf{v}_{f}-\mathbf{v}_{p}\right)+\left(1-\varepsilon_{f}\right) D_{v} \rho_{f} \frac{d}{d t}\left(\mathbf{v}_{f}-\mathbf{v}_{p}\right) \\
\frac{\partial}{\partial t}\left(\varepsilon_{p} \rho_{p} \mathbf{v}_{p}\right)+\nabla \cdot\left(\varepsilon_{p} \rho_{p} \mathbf{v}_{p} \mathbf{v}_{p}\right) & =-\varepsilon_{p} \nabla p-G \nabla \varepsilon_{f}+\nabla \cdot\left[\varepsilon_{p} \mu_{p}\left(\nabla \mathbf{v}_{p}+\nabla \mathbf{v}_{p}{ }^{T}\right)\right] \\
& +\varepsilon_{p} \rho_{p} \mathbf{g}+\beta\left(\mathbf{v}_{f}-\mathbf{v}_{p}\right)-\left(1-\varepsilon_{f}\right) D_{v} \rho_{f} \frac{d}{d t}\left(\mathbf{v}_{f}-\mathbf{v}_{p}\right)
\end{aligned}
$$

The enthalpy form is adopted for the energy conservation because the phase change occurs in the particle phase. The properties of particle phase vary continuously with temperature while the melting occurs in PCM. The energy equation can be solved over the entire domain. The enthalpy, density, thermal conductivity, and specific heat are all assumed to vary continuously, from their values in the solid to those in the liquid, as a 
function of temperature. The volume-averaged equations of energy conservation can be expressed by:

$$
\begin{aligned}
\frac{\partial}{\partial t}\left(\varepsilon_{f} \rho_{f} i_{f}\right)+\nabla \cdot\left(\varepsilon_{f} \rho_{f} i_{f} \mathbf{v}_{f}\right) & =-p\left[\frac{\partial \varepsilon_{f}}{\partial t}+\nabla \cdot\left(\varepsilon_{f} \mathbf{v}_{f}\right)\right]+\nabla \cdot\left(\varepsilon_{f} k_{e f f, f} \nabla T_{f}\right) \\
& -h\left(T_{f}-T_{p}\right) \\
\frac{\partial}{\partial t}\left(\varepsilon_{p} \rho_{p} i_{p}\right)+\nabla \cdot\left(\varepsilon_{p} \rho_{p} i_{p} \mathbf{v}_{p}\right) & =-p\left[\frac{\partial \varepsilon_{p}}{\partial t}+\nabla \cdot\left(\varepsilon_{p} \mathbf{v}_{p}\right)\right]+\nabla \cdot\left(\varepsilon_{p} k_{e f f, p} \nabla T_{p}\right) \\
& +h\left(T_{f}-T_{p}\right)
\end{aligned}
$$

The melting of particle phase that is in the encapsulated particles does not cause mass transport between the particle phase and fluid phase. Therefore, the terms related to the mass transfer between two phases vanish in the above governing equations. For the fluidparticle two-phase flow, the relationship between fluid and particle volume fraction is $\varepsilon_{f}+\varepsilon_{p}=1$. Therefore, only one of the volume fractions is independent. In the thermal energy equations, the dissipation function terms and the terms of Joule's heating and thermal radiation have been neglected. The continuum enthalpies of the fluid and particle phases can be expressed, respectively, as:

$$
\begin{aligned}
& i_{f}=c_{p, f, l} T_{f}+\left(c_{p, f, s}-c_{p, f, l}\right) T_{m, f}+h_{l s, f} \\
& i_{p}=c_{p, p} T_{p}+f_{p, l}\left[\left(c_{p, p, s}-c_{p, p, l}\right) T_{m, p}+h_{l s, p}\right]
\end{aligned}
$$

where $h_{l s}$ is the latent heat of fusion, $T_{m}$ is the melting temperature, and $f_{p, l}$ is the liquid mass fraction in the particle. The density, specific heat at constant pressure, and thermal conductivity of PCM particles are given, respectively, as follows:

$$
\begin{aligned}
& \rho_{p}=\xi_{p, l} \rho_{p, l}+\left(1-\xi_{p, l}\right) \rho_{p, s} \\
& c_{p, p}=f_{p, l} c_{p, p, l}+\left(1-f_{p, l}\right) c_{p, p, s}
\end{aligned}
$$




$$
k_{p}=\xi_{p, l} k_{p, l}+\left(1-\xi_{p, l}\right) k_{p, s}
$$

where $\xi_{p, l}$ is the liquid volume fraction in the particle. In the above treatment, the mass and thermal properties of capsule shell material are neglected.

To solve the complete set of governing equations, the following are chosen as the basic variables: fluid volume fraction, $\varepsilon_{f}$; pressure, $p$; three components of fluid velocity vector, $u_{f}, v_{f}, w_{f}$; three components of particle velocity vector, $u_{p}, v_{p}, w_{p}$; fluid enthalpy, $i_{f}$; and particle enthalpy, $i_{p}$. The closure of the set of governing equations requires the specification of the constitutive equations. This implies that all other variables in the governing equations must be specified in terms of the basic variables.

The formulation of particle-particle interaction modulus $G$ that Bouillard et al. (1989) presented is adopted, i.e.:

$$
G=-1.0 e^{100\left(0.45-\varepsilon_{f}\right)}
$$

The drag coefficient $\beta$ is calculated according to two ranges of fluid phase volume fraction. If $\varepsilon_{f}<0.8$, the drag coefficient $\beta$ is obtained from the Ergun equation as follows:

$$
\beta=150 \frac{\left(1-\varepsilon_{f}\right)^{2} \mu_{f}}{\varepsilon_{f} d_{p}{ }^{2}}+1.75 \frac{\left(1-\varepsilon_{f}\right) \rho_{f}}{d_{p}}\left|\mathbf{v}_{f}-\mathbf{v}_{p}\right|
$$

If $\varepsilon_{f} \geq 0.8$, the drag coefficient $\beta$ becomes:

$$
\beta=\frac{3}{4} C_{d} \frac{\varepsilon_{f}\left(1-\varepsilon_{f}\right)}{d_{p}} \rho_{f}\left|\mathbf{v}_{f}-\mathbf{v}_{p}\right| \mathcal{\varepsilon}_{f}^{-2.65}
$$


In the above equation, $\varepsilon_{f}^{-2.65}$ shows the effect due to the presence of other particles in the fluid and acts as a correction to the usual Stokes law for free fall of a single particle (Gidaspow, 1986). $C_{d}$ is related to the particle Reynolds number as (Rowe, 1961):

$$
C_{d}= \begin{cases}\frac{24}{R e_{p}}\left(1+0.15 R e_{p}^{0.687}\right), & R e_{p}<1000 \\ 0.44, & R e_{p} \geq 1000\end{cases}
$$

where

$$
R e_{p}=\frac{\varepsilon_{f} \rho_{f}\left|\mathbf{v}_{f}-\mathbf{v}_{p}\right| d_{p}}{\mu_{f}}
$$

In Eqs. (2.3) and (2.4), $D_{v}$ is the virtual mass coefficient. For dispersed spherical particles:

$$
D_{v}=0.5
$$

The effective thermal conductivities are calculated using the approximate model presented by Bauer and Schlunder (1978):

$$
\begin{aligned}
& k_{e f f, f}=k_{b, f} / \mathcal{E}_{f} \\
& k_{e f f, p}=k_{b, p} / \varepsilon_{p}
\end{aligned}
$$

where

$$
\begin{aligned}
& k_{b, f}=\left(1-\sqrt{1-\varepsilon_{f}}\right) k_{f} \\
& k_{b, p}=\sqrt{1-\varepsilon_{f}}[\eta A+(1-\eta) \mathrm{Z}] k_{f} \\
& \mathrm{Z}=\frac{2(B-B / A)}{(1-B / A)^{3}} \ln \left(\frac{A}{B}\right)-\frac{2(B-1)}{(1-B / A)^{2}}-\frac{B+1}{1-B / A}
\end{aligned}
$$




$$
B=1.25\left(\frac{1}{\varepsilon_{f}-1}\right)^{10 / 9}
$$

For spherical particles:

$$
\begin{aligned}
& A=k_{p} / k_{f} \\
& \eta=7.26 \times 10^{-3}
\end{aligned}
$$

The volumetric heat transfer coefficient $h$ is obtained from:

$$
h=\frac{6\left(1-\varepsilon_{f}\right)}{d_{p}} h_{p}
$$

In the above equation, the heat transfer coefficient $h_{p}$ is estimated based on Wakao and Kagei (1982):

$$
N u_{p}=\frac{h_{p} d_{p}}{k_{f}}=2+1.1 \operatorname{Re}_{p}^{0.6} \operatorname{Pr}^{1 / 3}
$$

The particle phase temperature is determined as:

$$
T_{p}= \begin{cases}\frac{i_{p}}{c_{p, p, s} ;} & i_{p}<c_{p, p, s} T_{m} \\ T_{m, p} ; & c_{p, p, s} T_{m, p} \leq i_{p} \leq c_{p, p, s} T_{m, p}+h_{l s, p} \\ \frac{i_{p}-\left(c_{p, p, s}-c_{p, p, l}\right) T_{m, p}-h_{l s, p}}{c_{p, p, l}} ; & i_{p}>c_{p, p, s} T_{m, p}+h_{l s, p}\end{cases}
$$

Finally, the liquid mass fraction in the melting particle can be calculated from:

$$
f_{p, l}= \begin{cases}0 ; & T_{p}<T_{m, p} \\ \frac{i_{p}-c_{p, p, s} T_{m, p}}{h_{l s, p}} ; & T_{p}=T_{m, p} \\ 1 ; & T_{p}>T_{m, p}\end{cases}
$$


The liquid volume fraction in the melting particle can be calculated from:

$$
\xi_{p, l}=\frac{f_{p, l} \rho_{p, s}}{f_{p, l} \rho_{p, s}+\left(1-f_{p, l}\right) \rho_{p, l}}
$$

The set of nonlinear, coupled, partial differential equations (2.1) to (2.6), supplemented with the constitutive equations and the initial and boundary conditions, cannot be solved analytically. Therefore, a numerical method must be used to obtain an approximate solution. Hao and Tao (2000) proposed a numerical method by employing the finite volume technique based on the SIMPLE algorithm to solve the present set of conservation equations in a cylindrical coordinate system. We will use this numerical method in simulation for the following sections.

\subsection{Performance Evaluation of Liquid Flow with PCM in Micro-channels}

This section will focus on the parametric study of optimal conditions where heat transfer is enhanced with an increase in fluid power necessary for pumping the PCM suspension flow. Most of the simulation is conducted under the condition of $\varepsilon_{p}=0.25$ and $\mathrm{Re}=90-600$ with octadecane as PCM.

\subsubsection{Fluid-particle suspension flow}

In the simulation, the fluid-particle two-phase flow consists of water as the carrier fluid and microencapsulated octadecane $\left(\mathrm{C}_{18} \mathrm{H}_{38}\right.$, with a melting temperature of $301 \mathrm{~K}$ and latent heat of $223 \mathrm{~kJ} / \mathrm{kg}$ ) as the PCM particles, with a melamine-formaldehyde resinous wall. The thermophysical data of water, octadecane, and shell material used for the computation have been summarized in Table 2.1. The thermophysical properties are assumed to be independent of temperature in solid and liquid. The encapsulated PCM particles will maintain constant size during the phase change process. 
Table 2.1 Thermophysical properties of octadecane PCM suspension flow with shell materials.

\begin{tabular}{|l|c|c|c|c|c|}
\hline & Density & $\begin{array}{c}\text { Specific } \\
\text { Heat } \\
\mathrm{kg} / \mathrm{m}^{3}\end{array}$ & $\begin{array}{c}\text { Thermal } \\
\text { Conductivity } \\
\mathrm{W} /(\mathrm{m} \cdot \mathrm{K})\end{array}$ & $\begin{array}{c}\text { Latent } \\
\text { Heat } \\
\mathrm{kJ} / \mathrm{kg}\end{array}$ & $\begin{array}{c}\text { Viscosity } \\
\mathrm{Pa} \cdot \mathrm{s}\end{array}$ \\
\hline Water (273K) & 997.07 & 4179.6 & 0.606 & & $8.904 \times 10^{-4}$ \\
\hline $\begin{array}{l}\text { PCM particle with shell } \\
\text { (Liquid) }\end{array}$ & 936 & 2014 & 0.144 & & \\
\hline $\begin{array}{l}\text { PCM particle with shell } \\
\text { (Solid) }\end{array}$ & 1000 & 1754 & 0.310 & 167 & \\
\hline $\begin{array}{l}\text { Shell material } \\
\text { (Melamine-formaldehyde) }\end{array}$ & 1490 & 1670 & 0.420 & & \\
\hline
\end{tabular}

The viscosity of PCM particle, $\mu_{p}$, has to be defined, since the particle is considered as a continuum in the present model. The correlation proposed by Vand (1945) is extended to the study of the microencapsulated phase change suspensions as follows:

$$
\frac{\mu_{b}}{\mu_{f}}=\left(1-\varepsilon_{p}-A \varepsilon_{p}^{2}\right)^{-2.5}
$$

where $\mu_{b}$ and $\mu_{f}$ are the viscosities of the slurry (not of the solid phase only) and the carrier fluid, respectively. The constant, $A$, which depends upon the shape and rigidity of the particles, can be determined experimentally based on the pressure drop measurement. The bulk viscosity, $\mu_{b}$, is obviously not the same as the particle viscosity, $\mu_{p}$, in a thermophysical sense. In fact, due to the lack of experimental data, the viscosity $\mu_{p}$ has not been available for the system of liquid-solid two-phase flow. As a first degree of approximation, the value of $0.01 \mathrm{~Pa} \cdot \mathrm{s}$ for the particle viscosity, $\mu_{p}$, is used in the present study based on Hao and Tao (2004). A further discussion on the influence of the particle viscosity $\mu_{p}$ and its relation to the bulk viscosity $\mu_{b}$ will be presented in section 2.5 . 


\subsubsection{Geometry description}

A tube with the typical diameter of $D=1.22 \times 10^{-4} \mathrm{~m}$ is used in our computation based on the design of Tao et al. (2003). The tube length of $L=1.22 \times 10^{-2} \mathrm{~m}$ $(L / D=100)$ is used to ensure that the supplied PCM particles completly melt at the exit

of the tube. The PCM particles are spherical with the diameter of $d_{p}=6.3 \times 10^{-6} \mathrm{~m}$ and initially all in the solid phase when they enter the tube. It is assumed that the uniform suspension enters the tube at a temperature below the phase change temperature, and the surface heat flux on the sidewall is constant.

By neglecting the effect of gravity, the problem becomes steady and axisymmetrical. The radial and axial dimensions of the main hydrodynamic computational grids are respectively $\Delta r=3.05 \times 10^{-6} \mathrm{~m}$ and $\Delta z=2.44 \times 10^{-5} \mathrm{~m}$. The total number of computational grid nodes including the subnodes next to the heated wall and entrance is equal to $29 \times 509=14761$.

All of the equations and parameters are nondimensionalized before they are solved by the numerical code on a Sun Blade 1000 workstation. The convergence criterion is that the relative changes of the variables between two successive iterations are less than $10^{-6} \%$.

Development of the field configurations through time takes place in a sequence of time steps. At each time step the computation is accomplished in such a way as to utilize the results developed in the previous time step (or the initial conditions) for the calculation of new values of all field variables, and to store these in the computer in such a way that they can be processed yet again in the following cycle. Considering the 
balance between the computing efficiency and accuracy/stability, we choose the typical time steps between 0.001 and $0.01 \mathrm{~s}$, where the small time step corresponds to relatively high velocity.

\subsubsection{Boundary conditions}

The hydrodynamic boundary conditions are defined as follows: The centerline of the tube is modeled as an impermeable slip boundary conditions for both phases. The tube wall is modeled as impermeable, non-slip rigid surface for both phases. At the entrance, the influx of carrier fluid with a given Reynolds number based on the tube diameter is prescribed and the particles have the same inlet velocity as that of the fluid. At the exit, the continuity outflow boundary conditions are assumed for both phases.

For thermal boundary conditions: The centerline of the tube is considered to be an adiabatic surface for both phases. At the tube wall, a constant heat flux is imposed. At the entrance, the inlet temperature of carrier fluid and the temperature of the PCM particles with $f_{p, l, i n}=0$ are prescribed as $T_{f, \text { in }}=T_{p, i n}=295 \mathrm{~K}\left(<T_{m, p}=301 \mathrm{~K}\right)$. At the exit, the continuity energy outflow boundary conditions are assumed for both phases.

\subsubsection{Results}

For a given kind of PCM particles, there should exist an optimal relation between the channel design parameters $L$ and $D$, particle volume fraction $\varepsilon_{p}$, Reynolds number Re, and the wall heat flux $q_{w}$. Two new parameters are defined for this analysis. One is the effectiveness factor, $\varepsilon_{e f f}$ :

$$
\varepsilon_{\text {eff }}=\frac{Q_{\text {slury }}}{Q_{\text {pure water }}}
$$


where

$$
\begin{aligned}
& Q_{\text {slurry }}=q_{w} A=q_{w} \pi D L \\
& Q_{\text {pure water }}=\dot{m} c_{p} \Delta T=\rho V \frac{\pi}{4} D^{2} c_{p} \Delta T
\end{aligned}
$$

As shown in the above equation, $\varepsilon_{\text {eff }}$ is defined as the ratio of the total heat transfer rate of the PCM suspension flow to the total heat transfer rate of pure water single-phase flow with the same temperature difference from the inlet to the exit. Therefore, $\varepsilon_{\text {eff }}$ can be used to evaluate the heat transfer enhancement of the PCM suspension flow, compared to the single-phase water flow. The other parameter is the performance index, $P I$ :

$$
P I=\frac{(Q / P)_{\text {slurry }}}{(Q / P)_{\text {pure water }}}
$$

where,

$$
\begin{aligned}
& P_{\text {slury }}=\Delta p V \frac{\pi}{4} D^{2} \\
& P_{\text {purewater }}=f \frac{\pi^{2}}{8} \rho L D V^{3} \\
& f=\frac{64}{\operatorname{Re}}
\end{aligned}
$$

here PI compares the ratio of total heat transfer rate to fluid flow power (pressure drop multiplied by volume flow rate) between the PCM suspension flow and the pure water single-phase flow.

Figures 2.2(a) - 2.2(c) show the distribution of carrier fluid temperature, PCM particle temperature, and liquid mass fraction inside of PCM particle. The calculation is under the condition of $\operatorname{Re}=167, T_{f, \text { in }}=T_{p, \text { in }}=295 \mathrm{~K}\left(<T_{m, p}=301 \mathrm{~K}\right), \varepsilon_{p}=0.25$, 
$f_{p, l, i n}=0$, and $q_{w}=50 \mathrm{~W} / \mathrm{cm}^{2}$. The heat transfer between the suspension and the wall occurs when it flows through the tube and the thermal boundary layers next to the sidewall. The PCM in the particles starts melting when the temperature of solid phase reaches the phase change temperature as shown in Figs. 2.2(b) and 2.2(c).

\section{Maximum effectiveness factor}

Figures 2.3(a) - 2.3(d) shows the effectiveness factor and performance index of the PCM suspension flow as a function of heat flux at different Reynolds numbers of 90, 167, 300 , and 600. The calculation is under the condition of $T_{f, \text { in }}=T_{p, \text { in }}=295 \mathrm{~K}$ $\left(<T_{m, p}=301 \mathrm{~K}\right), \varepsilon_{p}=0.25, f_{p, l, i n}=0$. As shown in these figures, by keeping $L, D$, and $\varepsilon_{p}$ constant, and for a given Reynolds number, there exists an optimal wall heat flux under which the $\varepsilon_{\text {eff }}$ value is a maximum. The reason for this phenomenon is the following: For a given Reynolds number, the fluid flow rate is constant. If the PCM particles are fully melted right at the exit under a specified wall heat flux, the maximum heat absorbing capacity of the PCM suspension flow occurs. This means that for the given Reynolds number, when other heat fluxes apply to the wall, the PCM particles are either completely melted before reaching the exit or remain partially frozen at the exit. This indicates that there exists a unique match between a design Reynolds number and wall heat flux in order to maximize the heat transfer enhancement of PCM slurry flow.

Compared with the pure water single-phase flow, the maximum heat absorbing capacity of the PCM suspension flow is about 2.2 times higher for $\operatorname{Re}=90$, i.e., the

maximum effectiveness factor, $\mathcal{E}_{\text {eff, max }}$, equals 2.2. As Re increases, $\varepsilon_{\text {eff, max }}$ decreases 


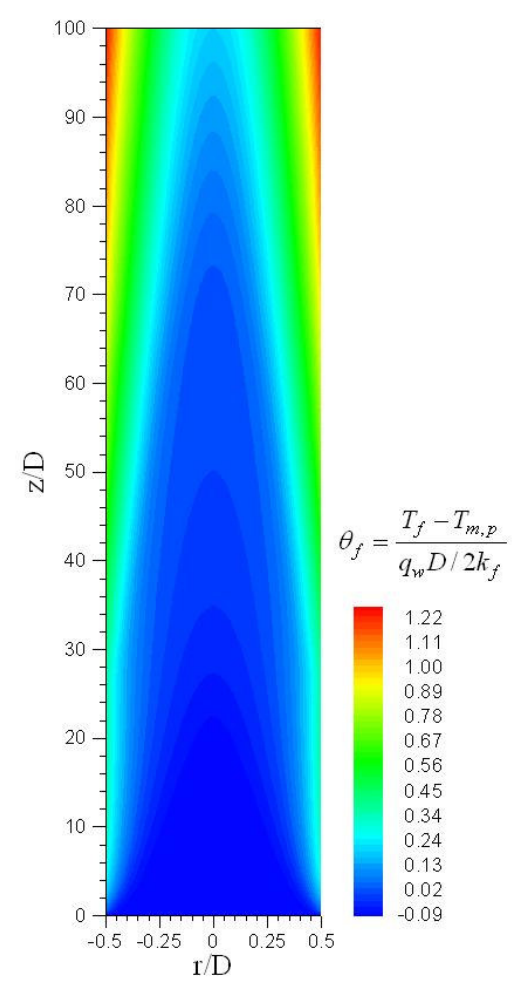

(a)

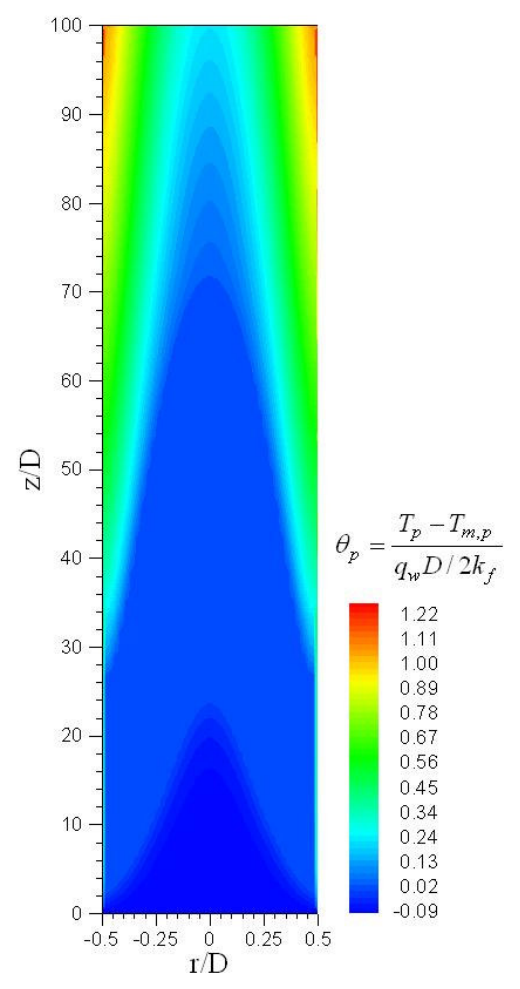

(b)

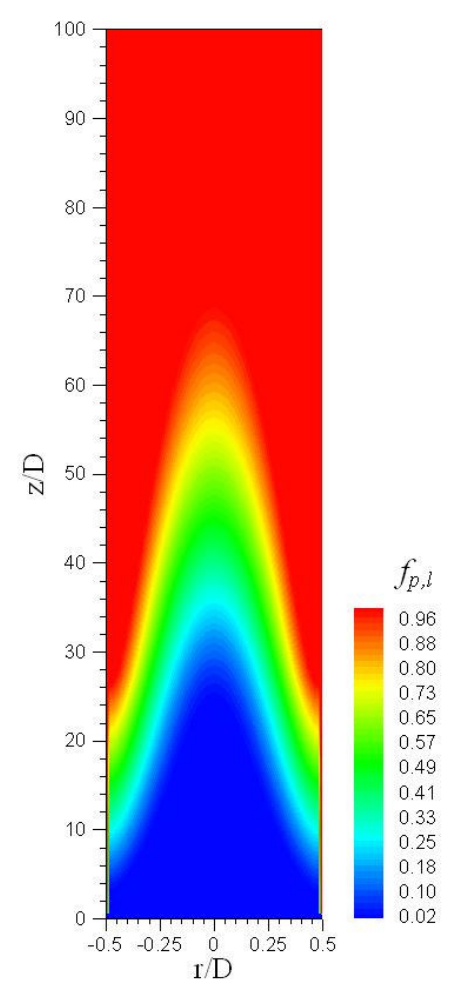

(c)

Figure 2.2 (a) Temperature of carrier fluid;

(b) Temperature of PCM particle;

(c) Liquid mass fraction inside of PCM particle.

slightly. At $\operatorname{Re}=600, \varepsilon_{e f f, \max }$ is 1.6 , as shown in Fig. 2.4. For $\operatorname{Re}=90, \varepsilon_{\text {eff, } \max }$ occurs at $q_{w, o p t}=10 \mathrm{~W} / \mathrm{cm}^{2}$; and for $\operatorname{Re}=600$, the corresponding $q_{w, o p t}=50 \mathrm{~W} / \mathrm{cm}^{2}$. Figure 2.4 is very useful for designing an optimal micro-channel heat exchanger.

\section{Performance index}

One of the important factors considered in making the design decisions of a microscale active liquid cooling devices is the pumping power requirement. The performance index $P I$ introduced in this study allows the designer to quantify and 


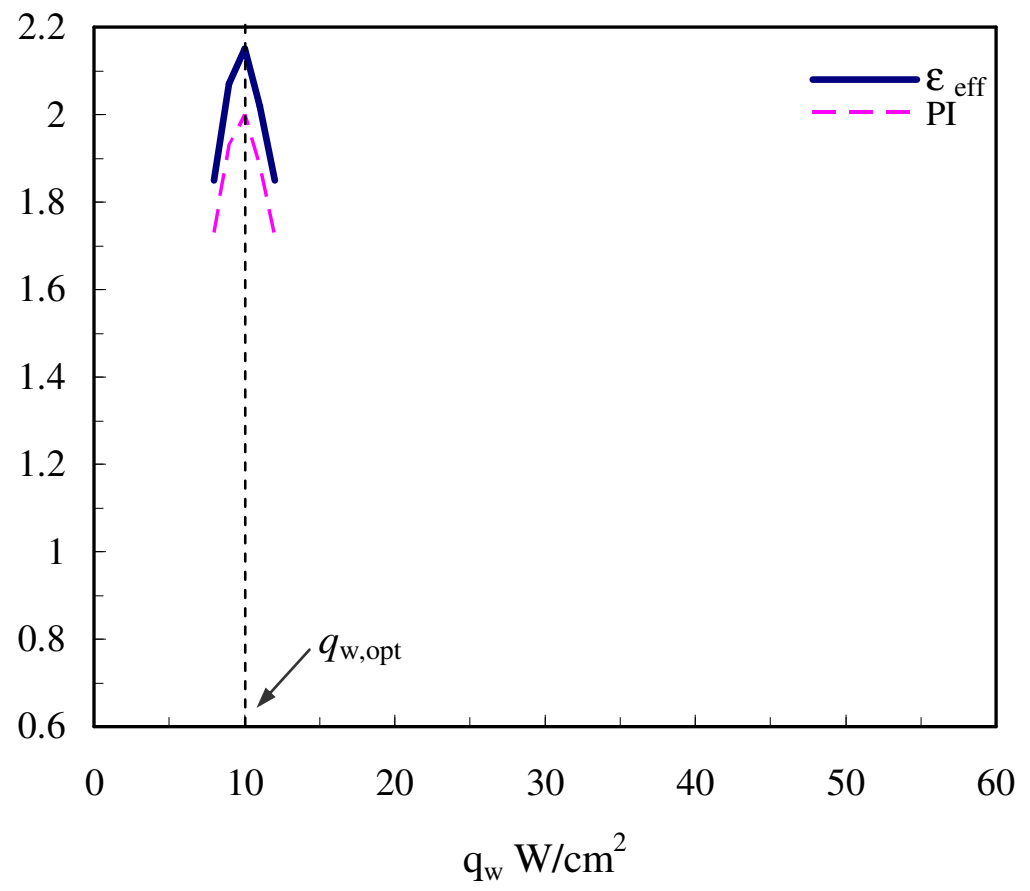

(a)

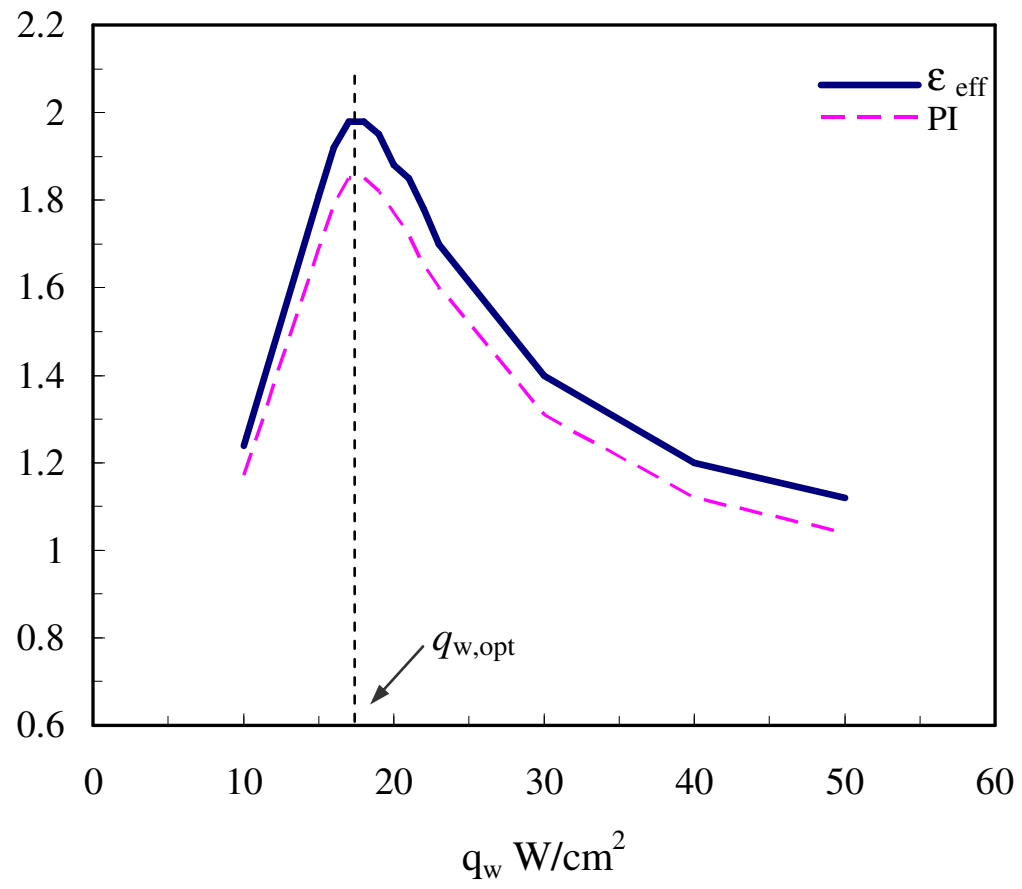

(b)

Figure 2.3 Effectiveness factor and performance index of slurry at different Reynolds numbers:

(a) $\operatorname{Re}=90$; (b) $\operatorname{Re}=167$. 


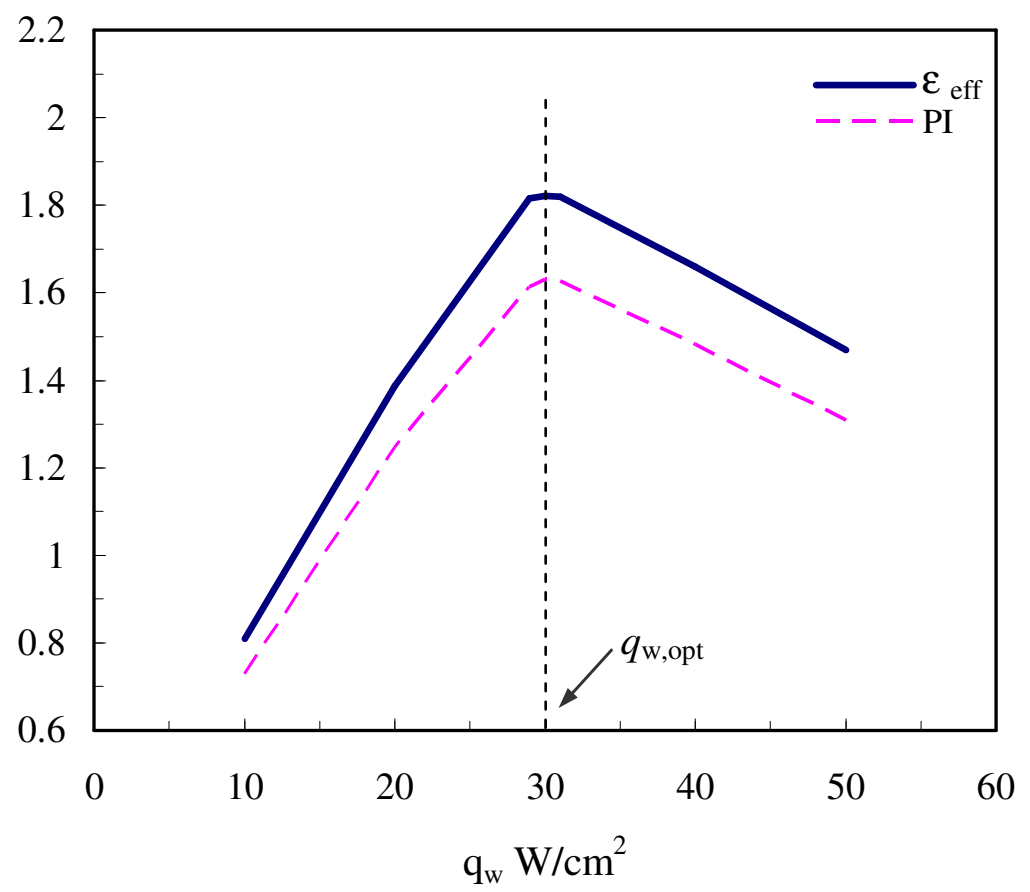

(c)

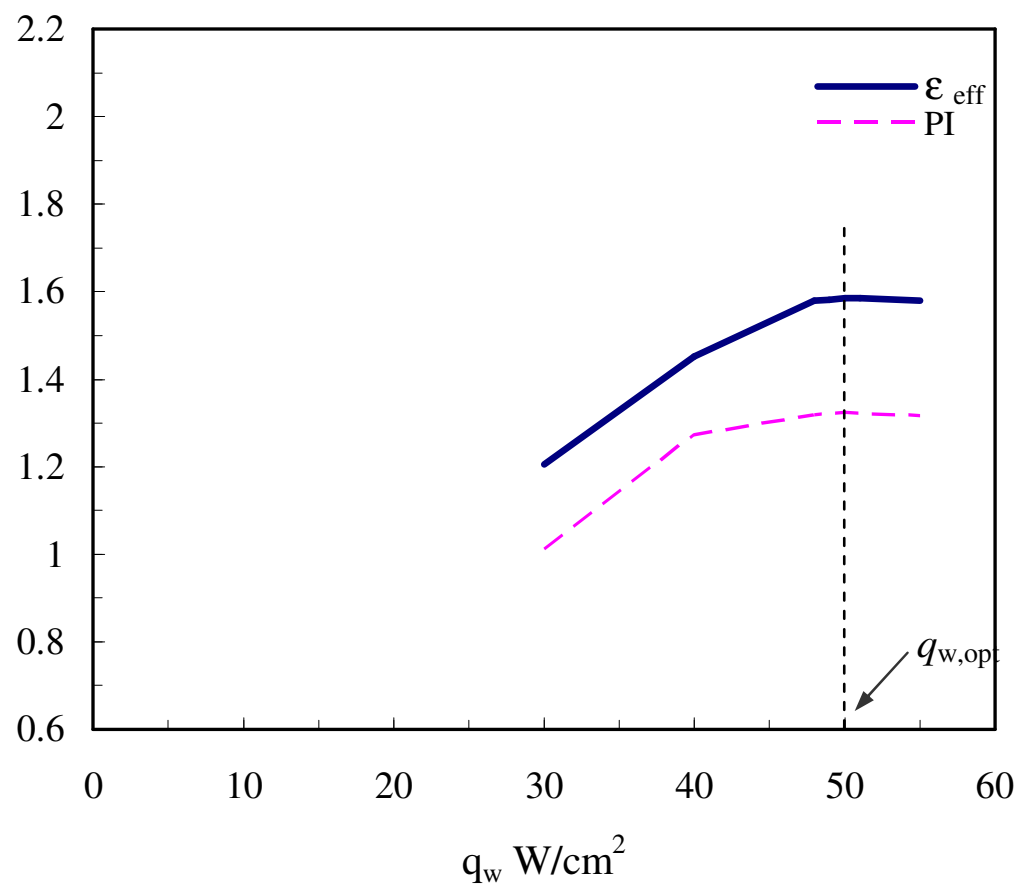

(d)

Figure 2.3 (Continued) Effectiveness factor and performance index of slurry at different Reynolds numbers:

(c) $\operatorname{Re}=300$; (d) $\operatorname{Re}=600$. 
compare their new designs. The results obtained in this study reveal that $P I$ can reach as high as 2.0 and follows the same trend as the effectiveness factor with the maximum value of $P I$ occurring at the same wall heat flux as that for $\varepsilon_{\text {eff, } \max }($ Figs. 2.3(a) $-2.3(\mathrm{~d}))$. This encouraging observation shows that the addition of PCM particles does not necessarily increase the pumping power required to move the flow. Because of the significant increases in heat transfer rate for a given pumping power, the required pumping power for a given heat transfer rate decreases ranging from 30 to $60 \%$ for the range of Re studied.

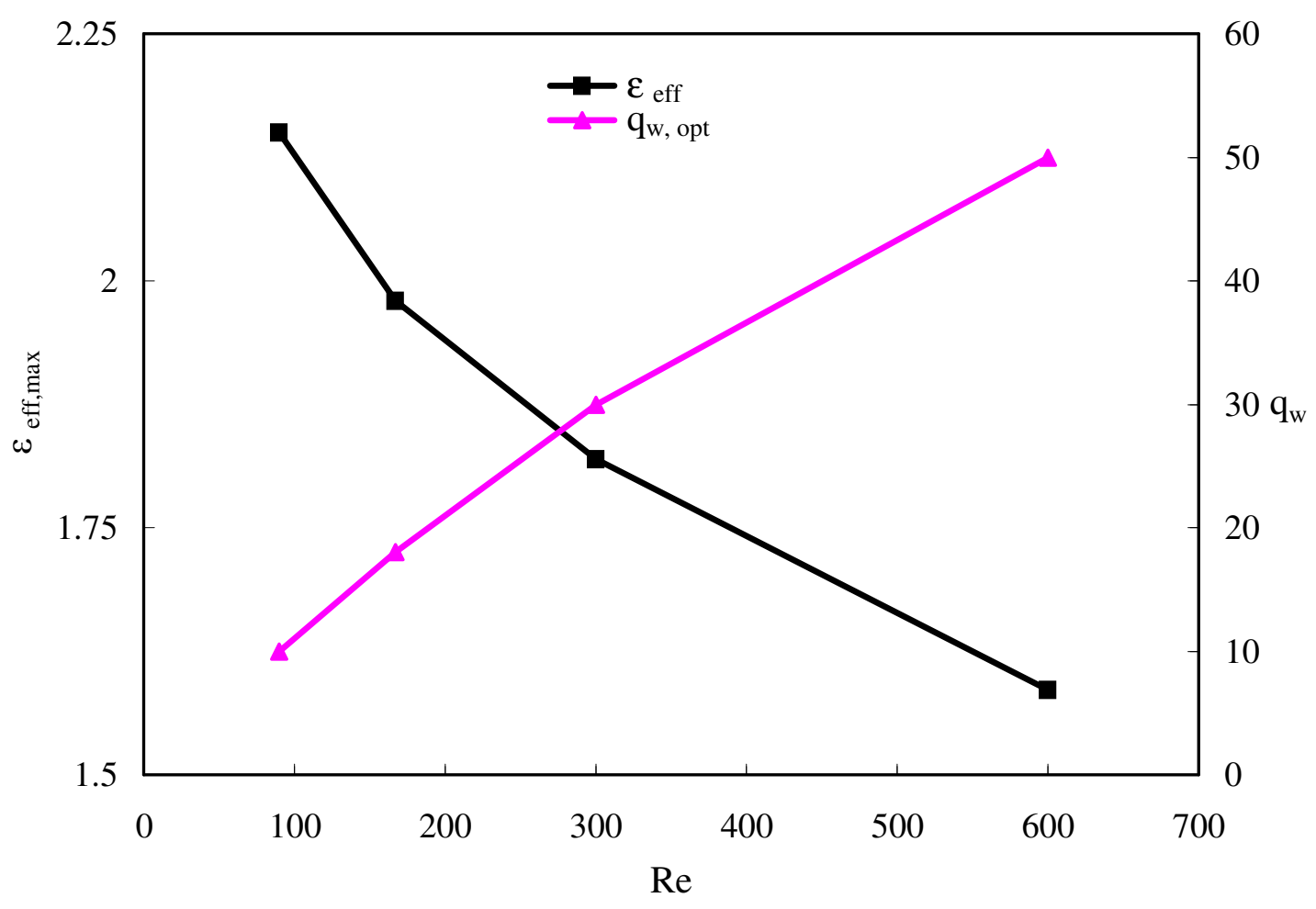

Figure 2.4 Maximum $\varepsilon_{\text {eff }}$ and corresponding $q_{w, o p t}\left(\mathrm{~W} / \mathrm{cm}^{2}\right)$ as a function of Re. 


\section{Heat transfer rate-to-pumping power ratio}

To further illustrate, Figs. 2.5(a) -2.5 (d) show the ratio of total heat transfer rate $Q$ to fluid flow power $P$ of the PCM suspension flow and the pure water single-phase flow vs. different wall heat flux and different Reynolds number, respectively. In general, the $Q / P$ ratio of the $\mathrm{PCM}$ suspension flow with phase change is significantly higher than the pure water single-phase flow. But in Fig. 2.5(c), at $\operatorname{Re}=300$, when $q_{w}$ less than $15 \mathrm{~W} / \mathrm{cm}^{2}$, the $Q / P$ ratio of the PCM suspension flow is even smaller than the pure water single-phase flow. The reason is that when $q_{w}$ less than $15 \mathrm{~W} / \mathrm{cm}^{2}$ at $\operatorname{Re}=300$, only a very small part of the PCM particles have melted at the exit of the tube. In this situation, the existence of PCM particles will not enhance heat transfer; it becomes a barrier to the fluid flow in the tube.

\section{Local heat transfer coefficient}

Figures 2.6(a) - 2.6(d) show the local heat transfer coefficient of the PCM suspension flow with phase change along the distance from the inlet, at a given wall heat flux and different Reynolds numbers. For comparison, the local heat transfer coefficient for the pure water single-phase flow is also shown in these figures. The local convection heat transfer coefficients are calculated from Newton's law of cooling:

$$
h=\frac{q_{w}}{T_{w}-T_{a v}}
$$

where $T_{a v}$ is the cross-sectional average temperature of the particle and the fluid. 


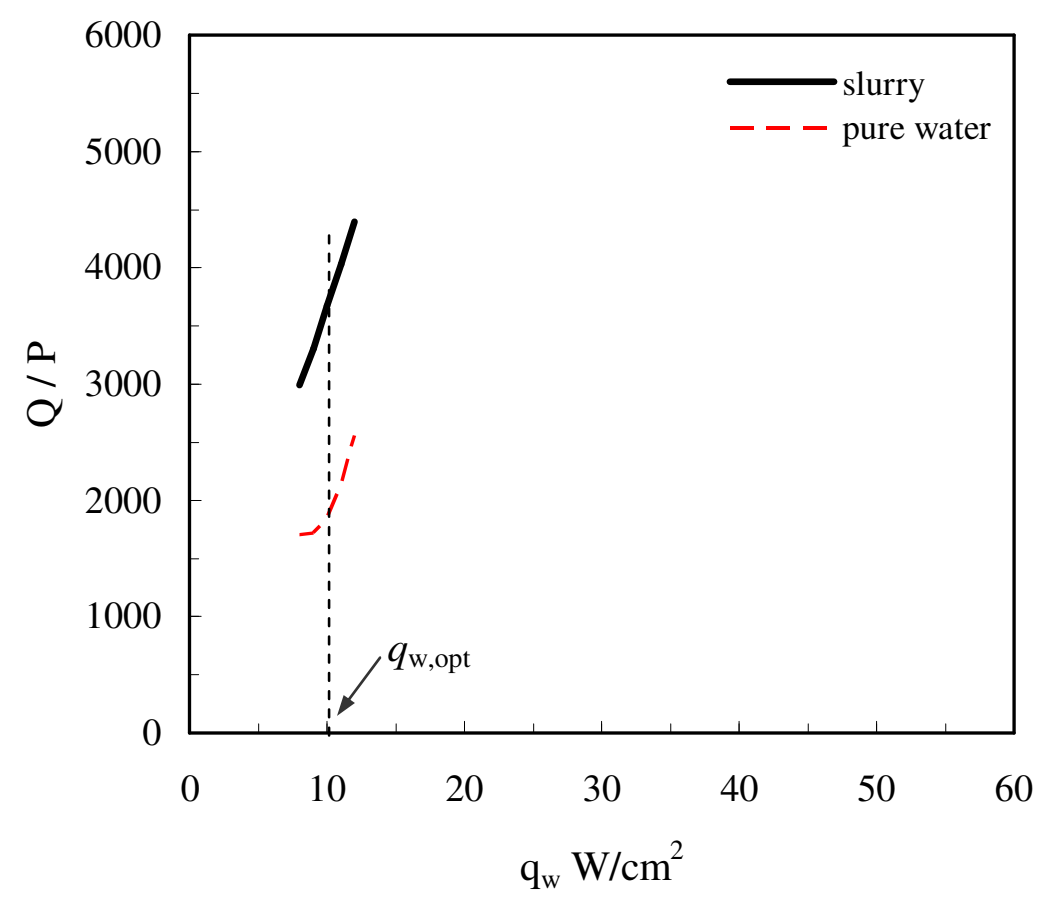

(a)

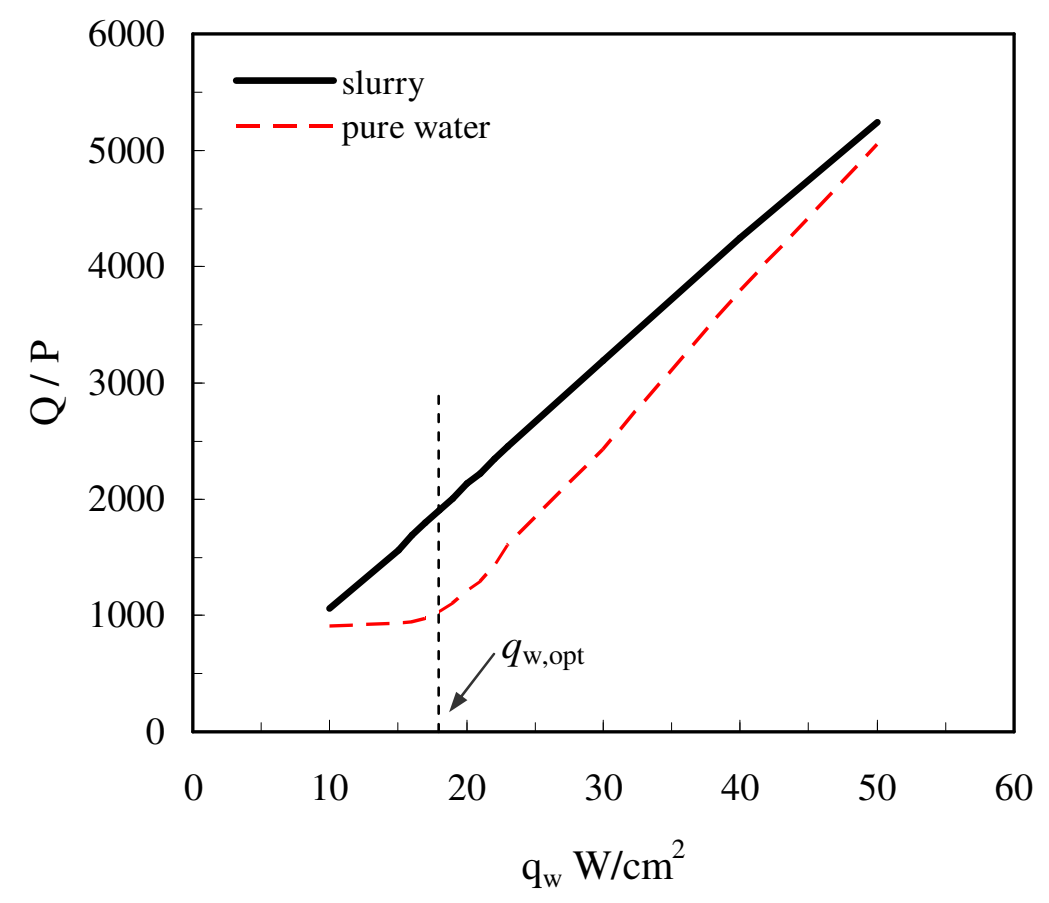

(b)

Figure 2.5 $Q / P$ ratio of slurry and pure water at different Reynolds numbers:

(a) $\operatorname{Re}=90$; (b) $\operatorname{Re}=167$. 


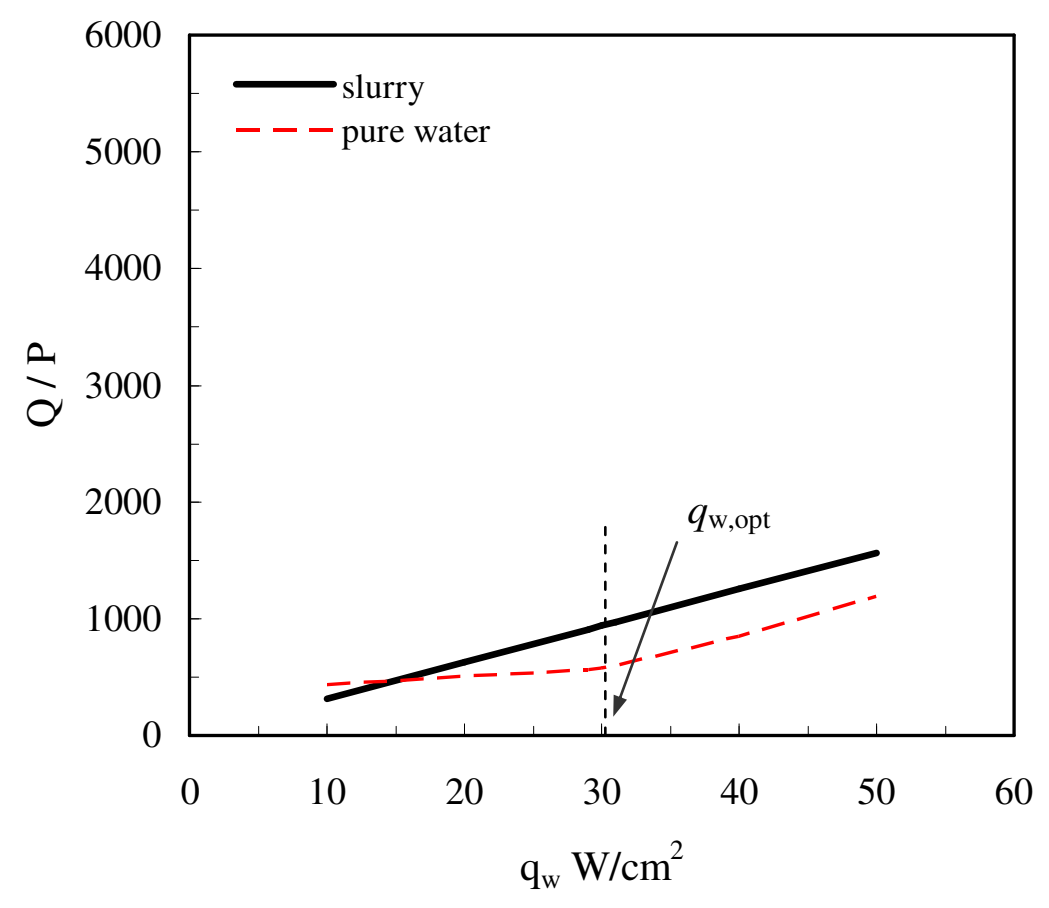

(c)

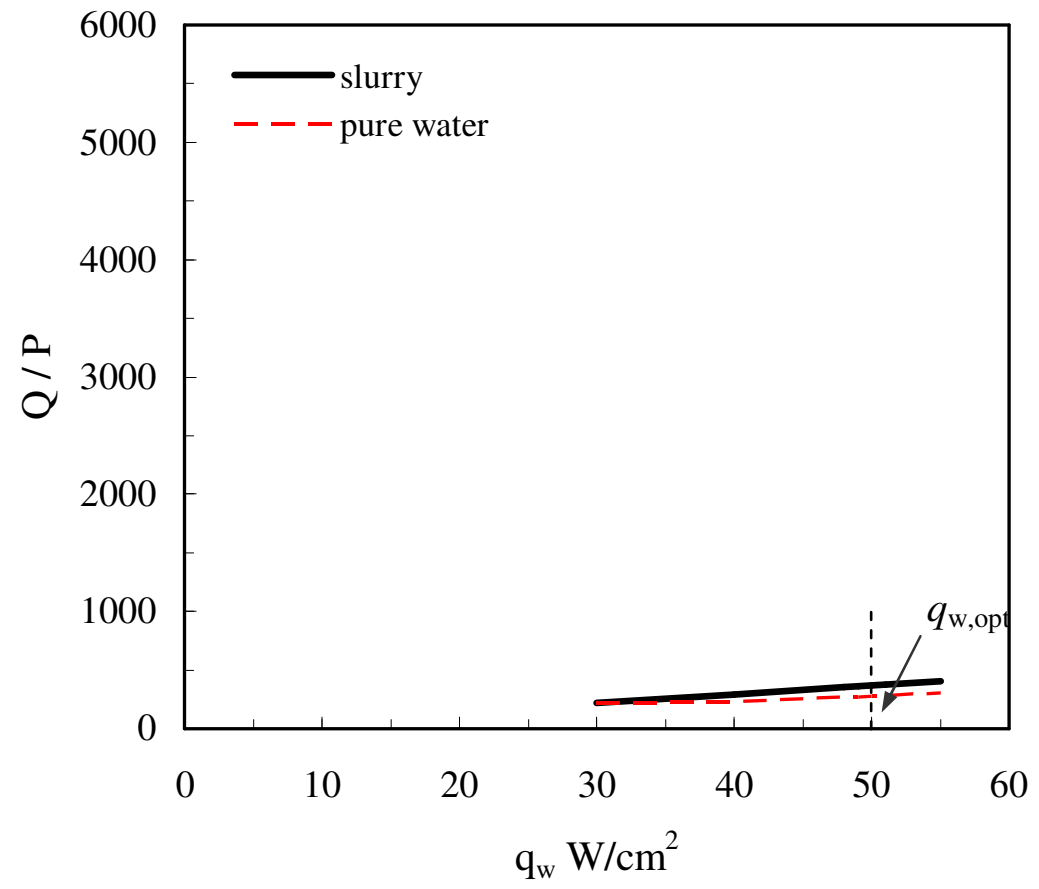

(d)

Figure 2.5 (Continued) $Q / P$ ratio of slurry and pure water at different Reynolds numbers:

(c) $\operatorname{Re}=300$; (d) $\mathrm{Re}=600$. 
From Figs. 2.6(a) - 2.6(d), we can see the significant effect of PCM particles on the heat transfer characteristics between the suspension and the wall. The temperature remains at the phase change temperature in the melting region, preventing the temperature and the thickness of thermal boundary layer from increasing. It causes the local heat transfer coefficient to increase in the melting region and reach the peak value at the location where the mean temperature of particle reaches the phase change temperature. The existence of a peak-value local heat transfer coefficient agrees very well with the experimental results presented in literature (Yamagishi et al., 1999; Choi et al., 1994). The results in Figs. 2.6(a) - 2.6(d) also show that the enhancement of the heat transfer mainly occurs in melting region when the suspension with PCM particles applies. In the earlier area of melting region and the melted region, the local heat transfer coefficient is even lower than that for pure water. This prediction also agrees very well with the experiments (Yamagishi et al., 1999; Choi et al., 1994).

Figure 2.7 shows the local heat transfer coefficient of the PCM suspension flow at different Reynolds numbers for a given wall heat flux. The peak value of the local heat transfer coefficient will go toward the exit and becomes higher with the increase of the Reynolds number. The reason is that with the increase of Re, the flow rate increases and the PCM particles need a longer distance to absorb enough heat to reach the phase change temperature.

\section{Particle volume fraction}

To consider the effect of particle volume fraction $\varepsilon_{p}$ on the heat transfer capacity of the PCM suspension flow, two cases are run by changing only the value of $\varepsilon_{p}$. The two 


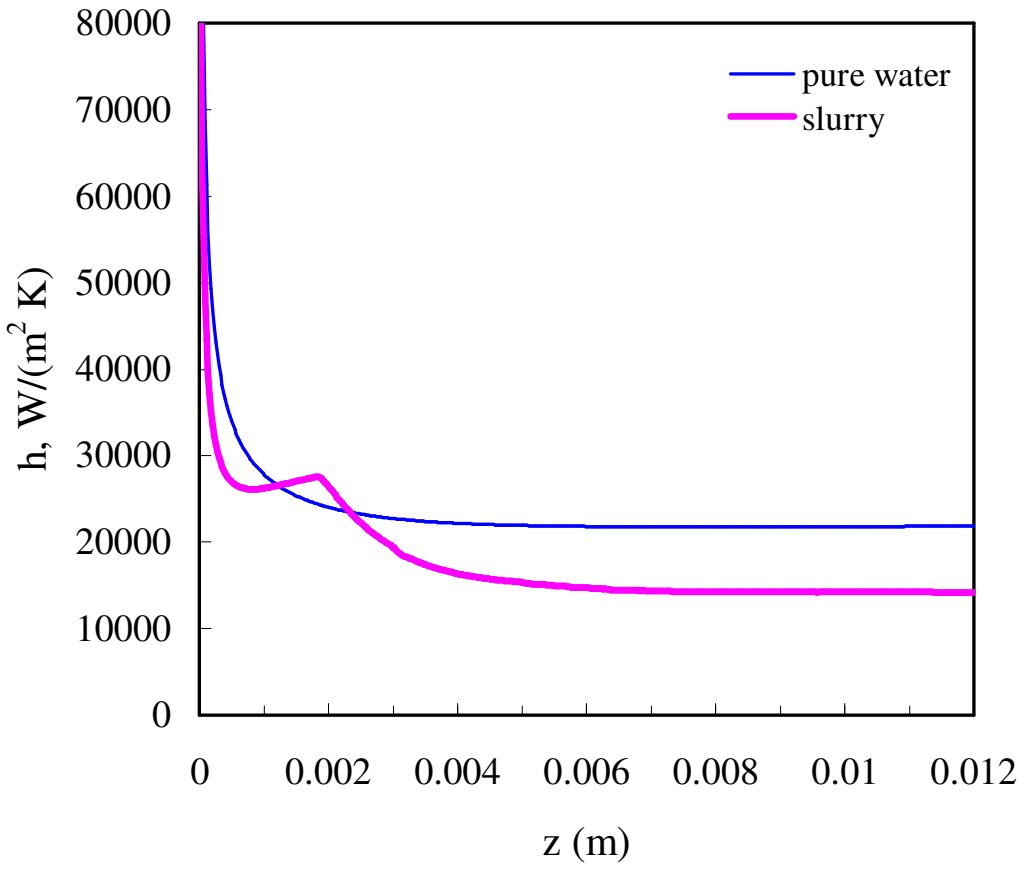

(a)

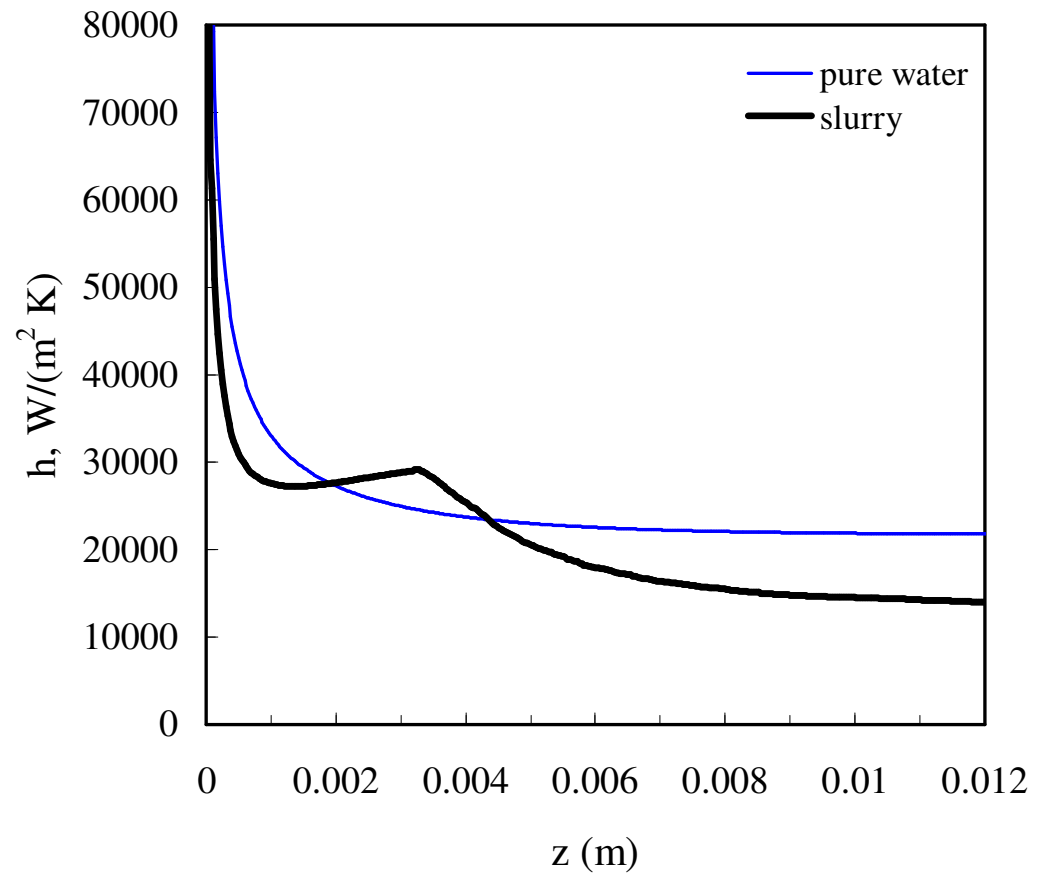

(b)

Figure 2.6 Local heat transfer coefficient along the channel at different Re with $q_{w}=50 \mathrm{~W} / \mathrm{cm}^{2}$ :

(a) $\operatorname{Re}=90$; (b) $\operatorname{Re}=167$. 


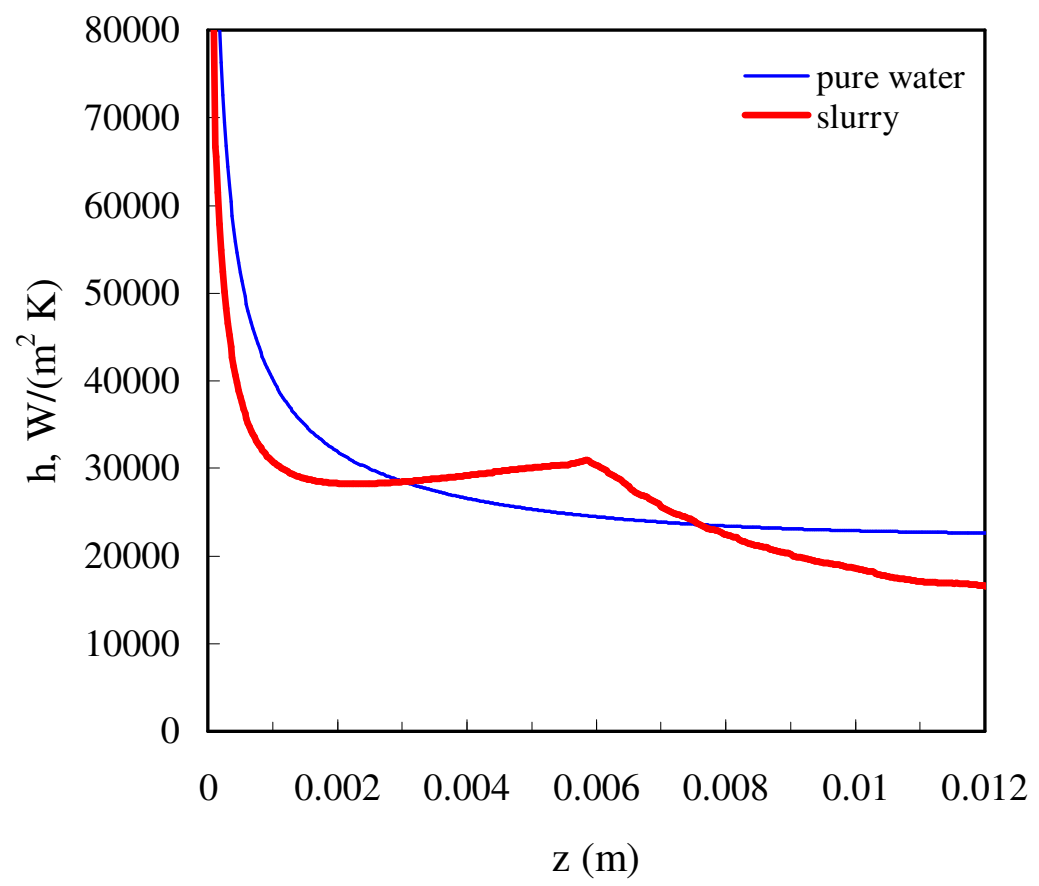

(c)

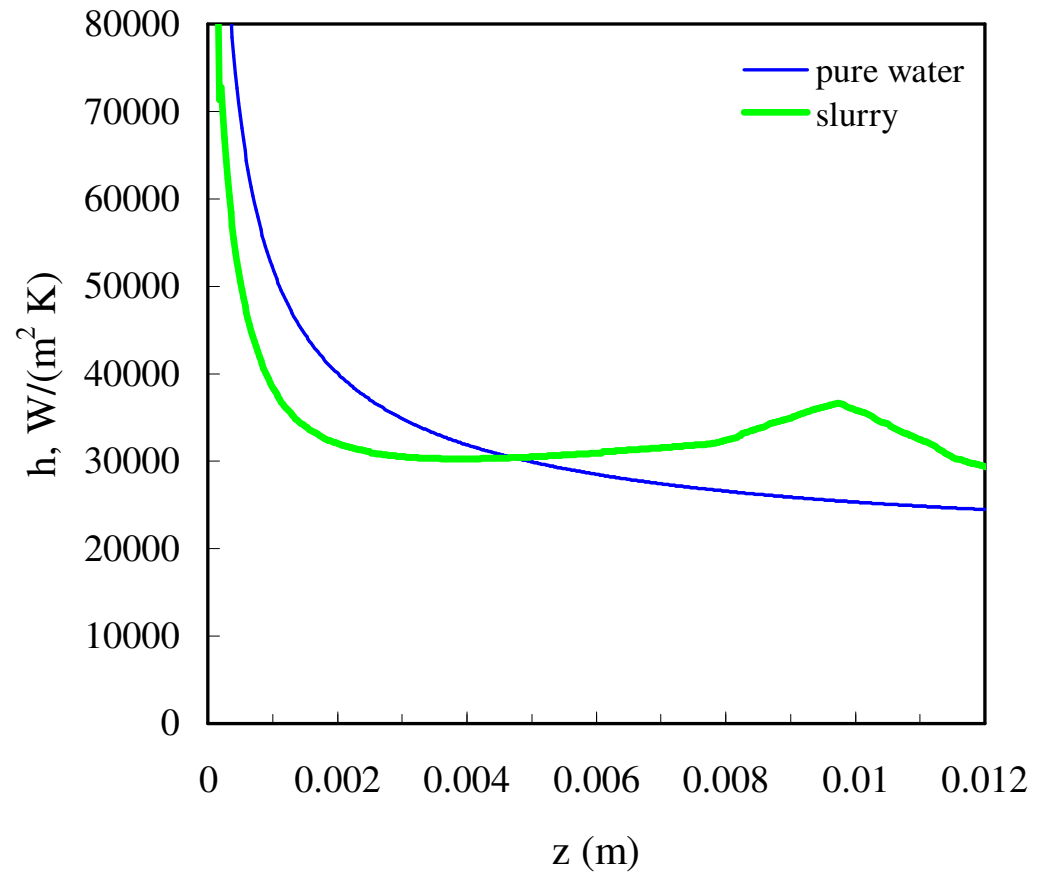

(d)

Figure 2.6 (Continued) Local heat transfer coefficient along the channel at different $\operatorname{Re}$ with $q_{w}=50 \mathrm{~W} / \mathrm{cm}^{2}$ :

(c) $\operatorname{Re}=300$; (d) $\operatorname{Re}=600$. 


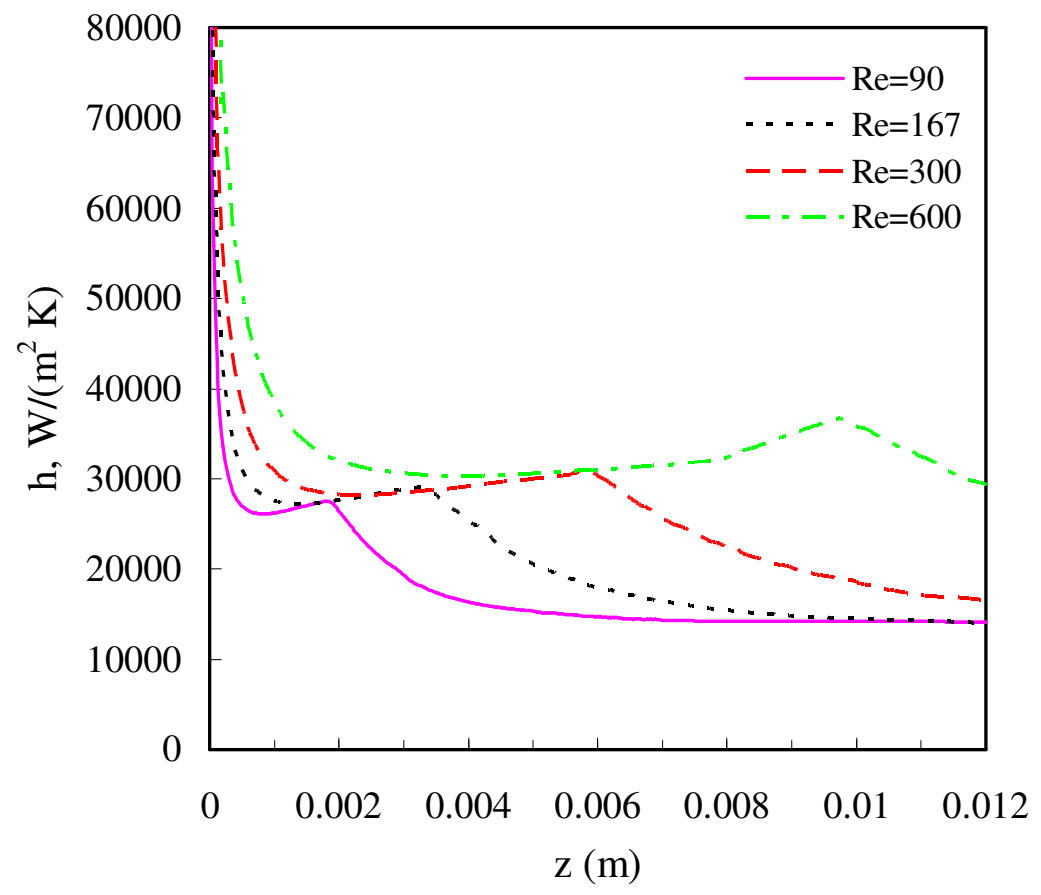

Figure 2.7 Distribution of local heat transfer coefficients where $\varepsilon_{e f f, \max }$ and $q_{w, \text { max }}$ occur at a given Re.

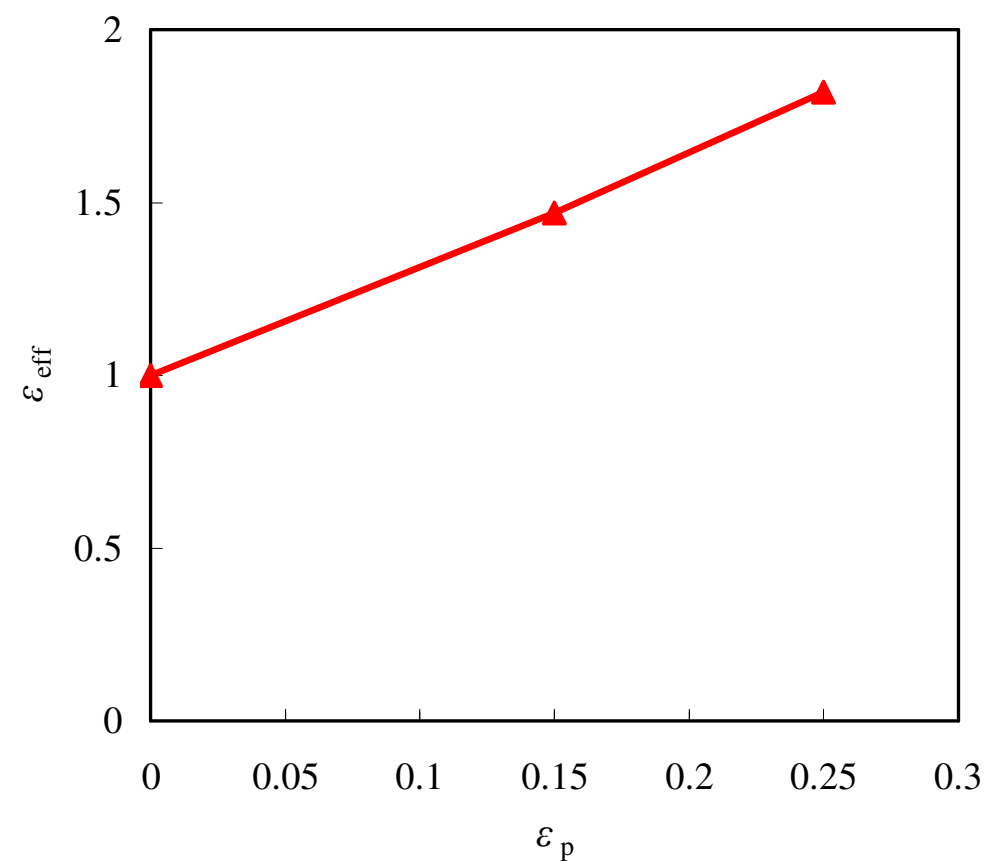

Figure 2.8 Effect of particle volume fraction on $\varepsilon_{\text {eff }}$ with $\operatorname{Re}=300, q_{w}=30 \mathrm{~W} / \mathrm{cm}^{2}$. 
cases are $\varepsilon_{p}=0.25$ and $\varepsilon_{p}=0.15$ for $\operatorname{Re}=300, q_{w}=30 \mathrm{~W} / \mathrm{cm}^{2}$. The change of effectiveness factor with particle volume fraction is shown in Fig. 2.8. From the figure, we can see that under given Reynolds number and given wall heat flux, the heat transfer enhancement of the PCM suspension flow will become higher if we increase the particle volume fraction. However the value of $\varepsilon_{\text {eff }}$ will not keep going up with $\varepsilon_{p}$, because too many particles will become a barrier to the fluid flow. Another parameter needed to be considered here is the viscosity of the PCM suspension flow $\varepsilon_{b}$, because by changing the particle volume fraction $\varepsilon_{p}$, the viscosity of the suspension flow is also changed. A detailed discussion of the influence of particle volume fraction will be presented in section 2.5 .

\section{Comparison with experimental results of macroscale slurry flow}

There has been no comparable experimental data for heat transfer involving PCM slurry flow in micro-channel. To evaluate the model's predictability, we conducted a simulation for a slurry flow with microencapsulated PCM suspension particles in a circular tube of $3 \mathrm{~mm}$ diameter since our model is still within the continuum theory domain. The experimental results for this case are available from (Goel et al., 1994). The PCM material used in the study of (Goel et al., 1994) is eicosane. The properties of eicosane are listed in Table 2.2. The test condition and comparison results are shown in Fig. 2.9, in which the dimensional temperature is presented as a function of the tube distance for two different Stephan numbers (Ste), which corresponds to two different wall heat fluxes. It was found that the wall temperature distribution is very sensitive to the inlet fluid temperature, which is near the phase change temperature of eicosane. 
Table 2.2 Thermophysical properties of eicosane PCM suspension flow.

\begin{tabular}{|l|c|c|c|c|c|}
\hline & Density & $\begin{array}{c}\text { Specific } \\
\text { Heat } \\
\mathrm{kg} / \mathrm{m}^{3}\end{array}$ & $\begin{array}{c}\text { Thermal } \\
\mathrm{J} / \mathrm{kg} \cdot \mathrm{K}) \\
\text { Conductivity } \\
\mathrm{W} /(\mathrm{m} \cdot \mathrm{K})\end{array}$ & $\begin{array}{c}\text { Latent } \\
\text { Heat } \\
\mathrm{kJ} / \mathrm{kg}\end{array}$ & Viscosity \\
$\mathrm{Pa} \cdot \mathrm{s}$
\end{tabular}

According to (Goel et al., 1994) where the original experimental results were reported in a dimensionless form, the inlet fluid temperature was controlled at a value slightly below the phase change temperature of eicosane, and no details on the controllability of the inlet temperature were given. Therefore three sets of simulation results will be presented for each of the two Stephan numbers (Fig. 2.9). They correspond to three slightly different inlet fluid temperatures, $T_{i n}=309 \mathrm{~K}, 309.85 \mathrm{~K}$, and $309.9 \mathrm{~K}$. It can be seen from Fig. 2.9 that the simulation results for $T_{\text {in }}=309.9 \mathrm{~K}$, a $0.05 \mathrm{~K}$ higher than the phase change temperature $\left(T_{m, p}=309.85 \mathrm{~K}\right)$, under which the PCM is in liquid phase at the inlet, agrees more favorably to the experimental results than the slightly lower inlet temperatures. Fig. 2.9(a) also shows that the simulated wall temperature for Ste $=2.0$ almost coincides with the measured one within experimental error bars. For the condition that the inlet slurry is at the phase change temperature $\left(T_{i n}=T_{m, p}=309.85 \mathrm{~K}\right)$ and with the PCM in solid phase, the simulated results clearly show the effect of phase change that yield a region where the variation of wall temperature remains flat. After the flow reaches near the exit, all the particles melt, and then the wall temperature starts to increase again, a response to the fluid temperature in the melting zone. If further reduce 


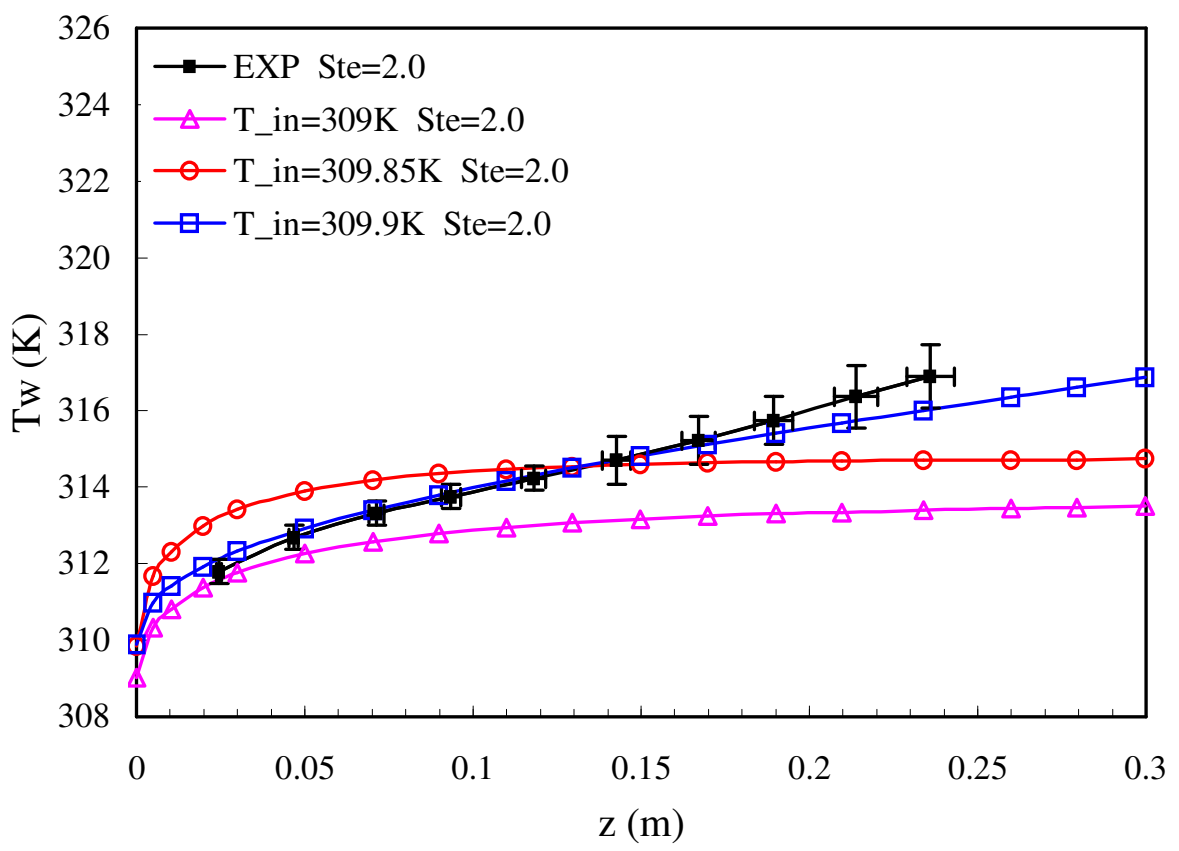

(a)

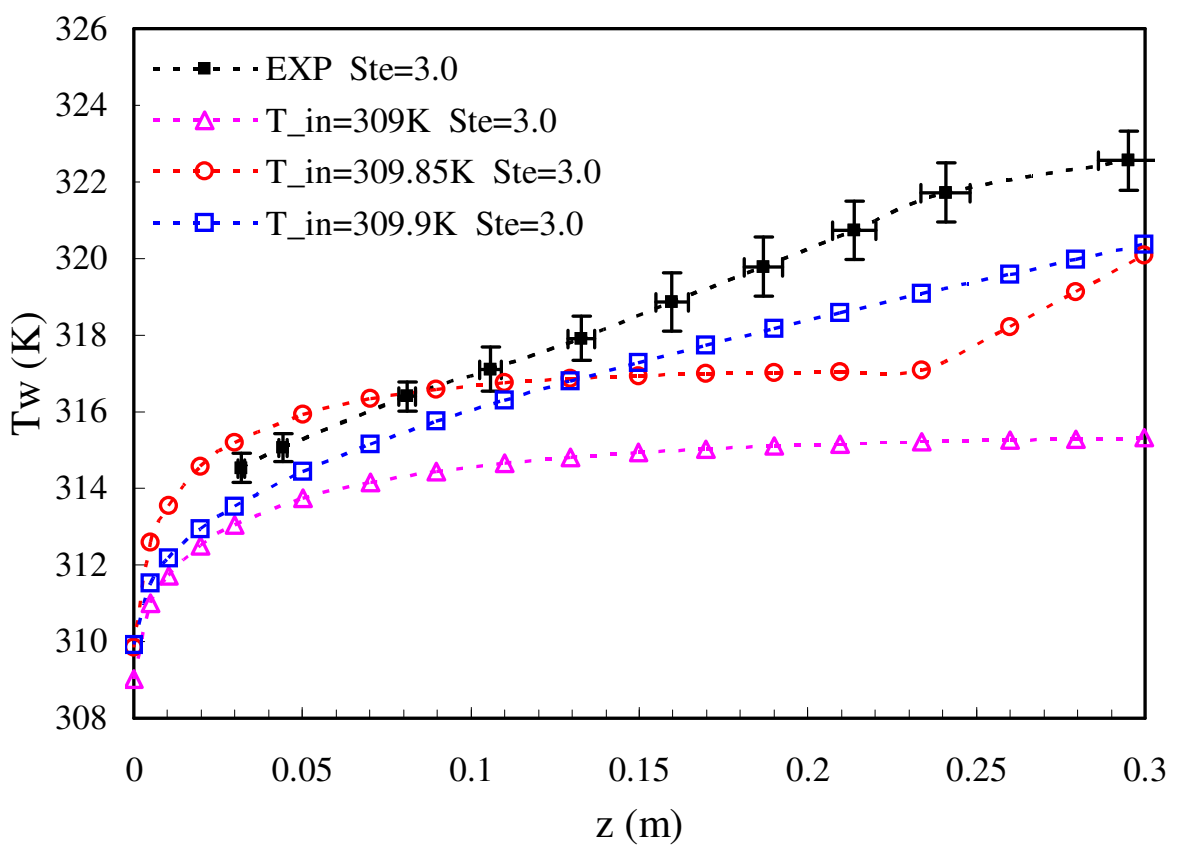

(b)

Figure 2.9 Comparison of simulation results with the experimental data under the following conditions: $\varepsilon_{p}=0.1, \operatorname{Re}=200, d_{p}=100 \mu \mathrm{m}$ :

(a) Ste $=2.0$; (b) Ste $=3.0$. 


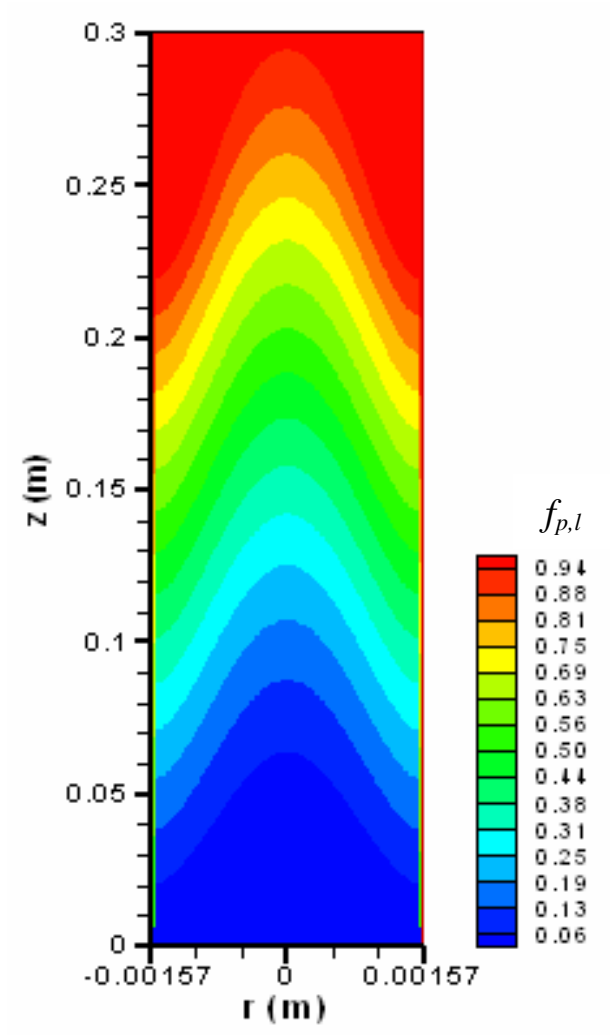

(a)

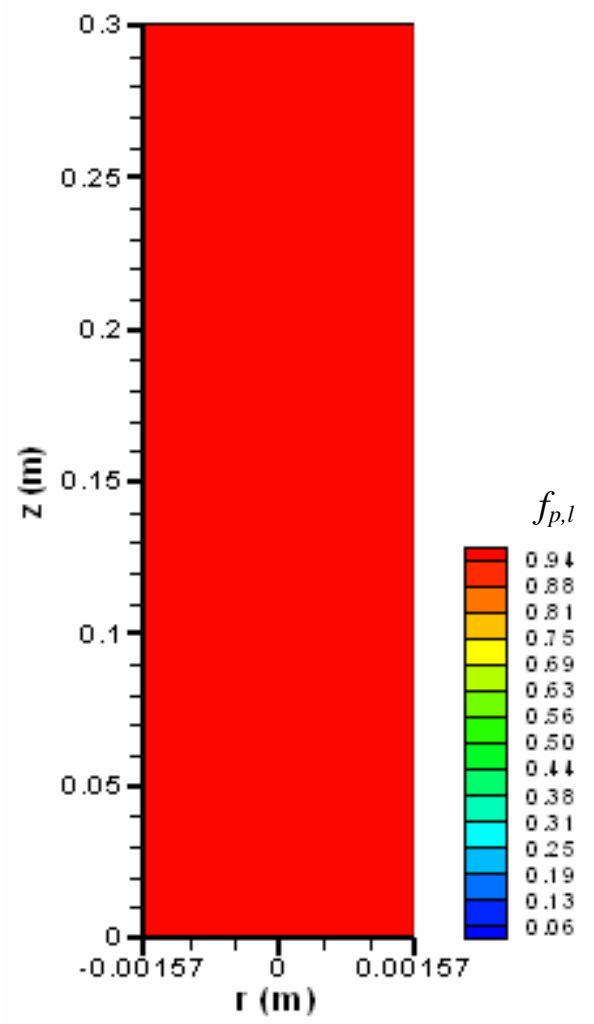

(b)

Figure 2.10 Liquid mass fraction inside of PCM particle with $\varepsilon_{p}=0.1, \operatorname{Re}=200, d_{p}=100 \mu \mathrm{m}$, Ste $=2.0$ :

(a) $T_{\text {in }}=309.85 \mathrm{~K}$; (b) $T_{\text {in }}=309.9 \mathrm{~K}$.

the inlet fluid temperature to $T_{\text {in }}=309 \mathrm{~K}$, a $0.85 \mathrm{~K}$ reduction, the wall temperature shows a low rate of increase and indicates that the entire tube is under the phase change region. As shown in Fig 2.10(a), the distribution of liquid mass fraction inside of PCM particle clearly indicates the gradual melting phenomena for $T_{\text {in }}=309.85 \mathrm{~K}$; while for $T_{\text {in }}=309.9 \mathrm{~K}$, PCM is all liquid throughout the tube (Fig. 2.10(b)). The above observation seems to suggest that the experiments presented in (Goel et al., 1994) may not be able to control the phase change region, which is very sensitive to the PCM slurry 
supply temperature. The experimentally determined wall temperature shows a trend that is similar to the post-melting region behavior that is characterized only by the liquid phase of the PCM.

It is also interesting to note from Fig. 2.9 that because the liquid $c_{p}$ value of eicosane (Table 2.2) is about $27 \%$ higher than that for solid; therefore, the suspension flow with initial liquid phase $\left(T_{i n}=309.9 \mathrm{~K}\right.$, which is $0.05 \mathrm{~K}$ above the melting point) results in a lower PCM/fluid temperature increase as compared to initially frozen (solid) PCM. In addition, for the same flow rate, the density difference between solid and liquid PCM could also contribute to the variation in particle velocity profiles; thus, affecting the local heat transfer coefficient. Because the wall temperature distribution follows the mean fluid temperature distribution, it is therefore shown that the wall temperature near the entrance for $T_{i n}=309.85 \mathrm{~K}$ is higher than that for $T_{i n}=309.9 \mathrm{~K}$.

To further validate the model, the simulation is done for polystyrene suspension flow for which experimental data are available (Ahuja, $1975^{1}$ and $1975^{2}$ ). In this case, no phase change occurs, and only heat transfer with suspension flow is considered. A constant wall temperature condition is assumed in the simulation to be the same as the heating fluid temperature in the experiment. This would over-predict the flow exit temperature. The suspension fluid is $50 \mu \mathrm{m}$ polystyrene spheres in $5.2 \%$ aqueous $\mathrm{NaCl}$ solution with the tube diameter of $0.001 \mathrm{~m}$, tube length of $0.55 \mathrm{~m}$, and particle concentration of $0.088 \%$. Our simulation results yield an exit mean fluid temperature of $44.12{ }^{\circ} \mathrm{C}$, versus the experimental result is $42.51{ }^{\circ} \mathrm{C}$, a $3.8 \%$ over prediction, as expected $\left(T_{\text {exit }}-T_{\text {in }}=42.3{ }^{\circ} \mathrm{C}\right)$. This validation is better than, or at least the same as, that 
presented in (Charunyakorn et al., 1991) with their majority of discrepancies ranging from $4 \%$ to $10 \%$.

The above comparison shows that the model presented in this study provides a more detailed, and reliable way to study the sensitivity of phase change behavior of a convective PCM slurry flow than the model that treats the slurry as a homogenous media with the modified heat capacity (Charunyakorn et al., 1991; Hu and Zhang, 2002). We further state that cautions should be taken in reporting the accuracy of any numerical simulation and experimental results involving PCM slurry flow where the phase change region needs to be quantified not only by the slurry temperature but also by the liquid content (liquid mass fraction, $f_{p, l}$ ) of PCM. It is obvious that further studies on experimental investigations of PCM flow in micro-channel are needed.

\subsubsection{Conclusions}

From the above discussion, the following conclusions may be drawn:

1. For a given Reynolds number and particle volume fraction, there exists an optimal wall heat flux $q_{w, o p t}$ that will yield a maximum effectiveness factor $\varepsilon_{\text {eff }, \text { max }}$. At the particle volume fraction of 0.25 , heat transfer can be enhanced by $60 \%$ to more than $110 \%$ with a Re between $90-600$.

2.For a given Reynolds number and particle volume fraction, there also exists a maximum performance index, $P I_{\max }$, at an optimal wall heat flux $q_{w, o p t}$. At the particle volume fraction of $0.25, P I_{\max }$ is between 1.3 and 2.0 for Re between $90-600$. It indicates that at the optimal condition, PCM flow not only significantly enhances heat 
transfer but also becomes more efficient, which means that less fluid pumping power are needed for a given heat transfer rate.

3. As Re increases, $\varepsilon_{\text {eff, max }}$ decreases. This indicates that to achieve the higher heat transfer rate by increasing $\mathrm{Re}$, the designer must be aware of the compromising of enhancement effects.

4. In general, the increase in the Reynolds number results in the decrease of heat transfer rate to pumping power ratio, $Q / P$. This is true for both PCM and single-phase flows in this study. Within the range of this study $\left(\varepsilon_{p}=0.25\right), Q / P$ is between 300 and 6,000 for $\operatorname{Re}=90-600$ with the high $Q / P$ corresponds to the low $\operatorname{Re}$. Therefore, to best take advantage of heat transfer enhancement for micro-channel flow with PCM under laminar conditions, it is recommended that a low Reynolds number condition is maintained.

5. The limited comparison of the presented model with an experiment for macroscale flow shows that the model results reasonably agrees with the experimental data for the condition of initial temperature at the $0.05 \mathrm{~K}$ above the phase change temperature. The model results indicate for the first time the sensitivity of the simulation results to the initial flow temperature that might explain the uncertainty in the reported experimental results in the literature. It also suggests that the evaluation on a model simulation for PCM slurry flow should consider the model's ability to quantify the phase change region characterized by both temperature and liquid/solid fractions.

The above conclusions will assist designers to make decisions that relate to the design or selection of a micro pump suitable for microscale heat transfer devices. 


\subsection{Low Reynolds Number Limit of Heat Transfer Enhancement with PCM Slurry Flow in Micro-channels}

There are many heat exchanger applications where the heat-transfer rate to fluid pumping power ratio has to be maximized to achieve both high thermal efficiency and cost effectiveness. Our numerical study in section 2.3 involving PCM particles in microchannels revealed significant heat transfer enhancement, provided that an optimal balance among the Reynolds number, particle size, tube length and heat flux is achieved. These findings, however, were limited to the concentration of less than $30 \%$. Therefore, in this section, we will focus on the heat transfer enhancement effects for very low Reynolds number $(\operatorname{Re}<50)$ and high PCM concentration slurry flow $(50 \%-70 \%)$.

Both numerical simulation and experimental validations are carried out in this section. The objective is to determine the range of optimal parameters for a higher $Q / P$ ratio at the very low Reynolds number and high PCM concentration conditions, which will be able to yield at least the same level of heat transfer enhancement as at the higher Reynolds numbers. A secondary objective is to use experimental data to investigate the validity of the numerical model, which was developed for microencapsulated particles, for emulsion PCM flow.

In the numerical simulation of this section, the fluid-particle suspension flow still consists of water as the carrier fluid and octadecane as the PCM particles encapsulated by

a melamine-formaldehyde resinous shell materials. So the same thermophysical property values provided in Table 2.1 will be used. The same micro-channel geometry, initial and boundary conditions given in section 2.3 by detail, will be inherited here. The only 
difference is that the octadecane PCM particle size is $d_{p}=5 \times 10^{-7} \mathrm{~m}$ in the current simulation, rather than $d_{p}=6.3 \times 10^{-6} \mathrm{~m}$ in the previous section.

The experimental data we used to validate our simulation results are provided by Tao et al. (2007). In the experiment, the fluid-particles two-phase flow consists of water as the carrier fluid and octadecane without shell materials as the PCM particles in emulsion form, which is different from the previous studies with encapsulated PCM particles. The average particle size of the emulsion is $d_{p}=2.9 \times 10^{-5} \mathrm{~m}$. The thermophysical data of water and octadecane without shell materials have been summarized in Table 2.3.

\subsubsection{Experiments}

Figure 2.11(a) shows a schematic diagram of the experimental setup used by Tao et al. (2007). The test section is a circular copper tube of $0.3 \mathrm{~m}$ in length ( $L=0.3 \mathrm{~m}$ ) with an inner diameter of $3.14 \mathrm{~mm}\left(D_{i n}=0.00314 \mathrm{~m}\right)$ and an outer diameter of $4.76 \mathrm{~mm}$ ( $D_{\text {out }}=0.00476 \mathrm{~m}$ ). Constant heat flux was maintained at the test section by winding insulated copper wire around the copper tube. A DC power supply was used to control

Table 2.3 Thermophysical properties of octadecane PCM slurry without shell materials.

\begin{tabular}{|ll|c|c|c|c|c|}
\hline & Density & $\begin{array}{c}\text { Specific } \\
\text { Heat } \\
\mathrm{kg} / \mathrm{m}^{3}\end{array}$ & $\begin{array}{c}\text { Thermal } \\
\mathrm{J} /(\mathrm{kg} \cdot \mathrm{K})\end{array}$ & $\begin{array}{c}\text { Latent } \\
\mathrm{W} /(\mathrm{m} \cdot \mathrm{K})\end{array}$ & $\begin{array}{c}\mathrm{V} \text { Viscosity } \\
\mathrm{kJ} / \mathrm{kg}\end{array}$ & $\mathrm{Pa} \cdot \mathrm{s}$ \\
\hline Water $(273 \mathrm{~K})$ & 997.07 & 4179.6 & 0.606 & & $8.904 \times 10^{-4}$ \\
\hline $\begin{array}{l}\text { PCM particle without shell } \\
\text { (Liquid) }\end{array}$ & 780 & 2200 & 0.150 & & \\
\hline $\begin{array}{l}\text { PCM particle without shell } \\
\text { (Solid) }\end{array}$ & 850 & 1800 & 0.340 & 223 & \\
\hline
\end{tabular}




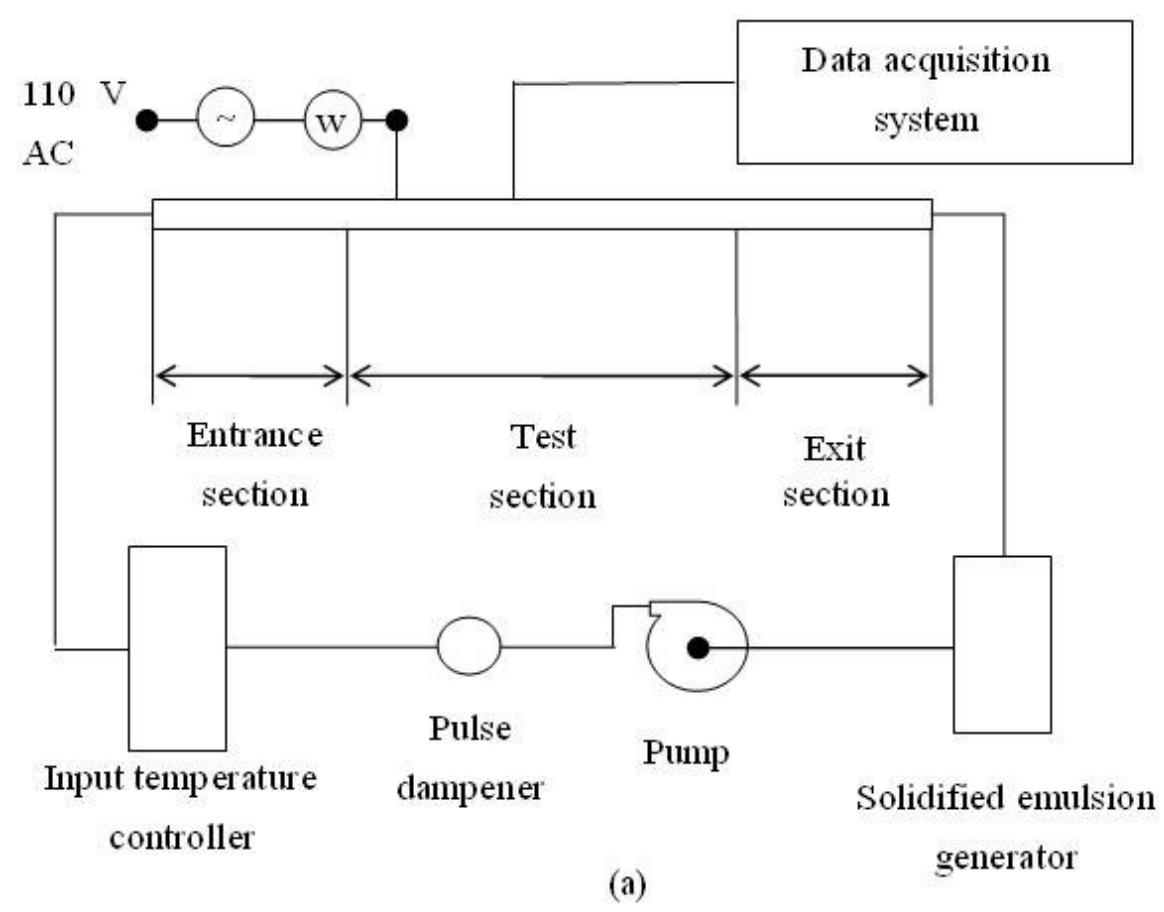

To the data logger

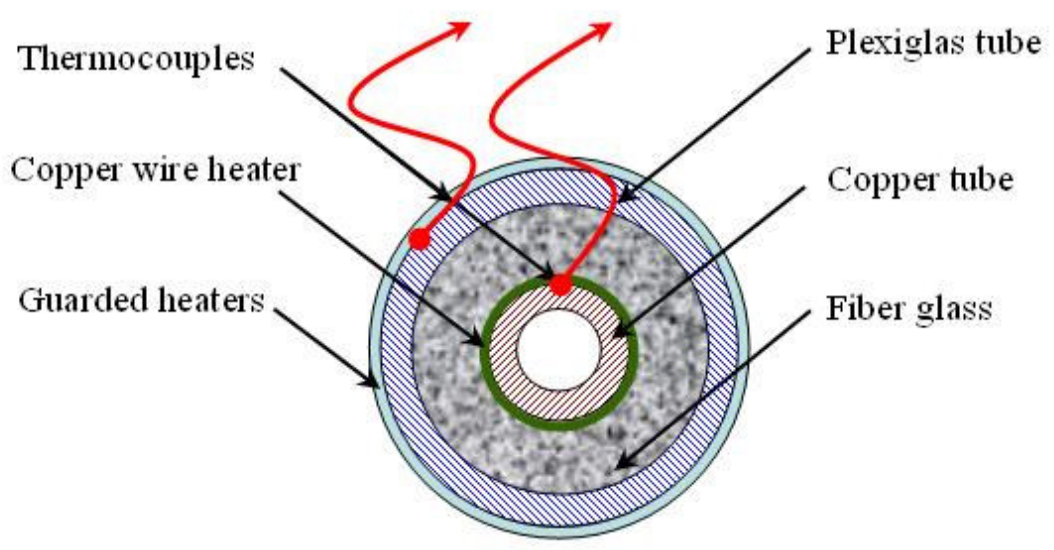

(b)

Figure 2.11 Schematic diagram of the experimental setup:

(a) Entire system;

(b) Cross-sectional view of the test section.

(Source: Tao et al., 2007) 
the voltage supplied to the wire and thus, heat input to the whole test section assembly. The dimensions of the Plexiglas tube are $31.75 \mathrm{~mm}$ in outer diameter, $3.175 \mathrm{~mm}$ in thickness, and $600 \mathrm{~mm}$ in length. The Plexiglas tube is covered with 4 flexible silicon rubber heaters of $4 \times 6$ inches $(0.1016 \mathrm{~m} \times 0.3048 \mathrm{~m})$ in size. The temperature between the surface of the Plexiglas and guarded heaters was measured at four locations along the length. To ensure the flow entering the test section is fully developed, an extended length of $150 \mathrm{~mm}$ is provided upstream of the test section. The cross section of the test section is shown in Fig. 2.11(b).

The emulsion is made in a container and then fed through a heat exchanger where the temperature of the emulsion goes below the re-crystallization temperature of the phase change material. The average particle size of the emulsion is 29 micrometers measured by using a microscopic video camera. The bath temperature of the heat exchanger is kept around $24{ }^{\circ} \mathrm{C} \pm 0.01{ }^{\circ} \mathrm{C}$, which helps in maintaining the temperature of the slurry at $25{ }^{\circ} \mathrm{C}$. At this temperature the slurry is pumped into the test section using a diaphragm pump. The geometrical data of this experimental apparatus for PCM emulsion is similar to the one used by Goel et al. (1994) for microencapsulated PCM suspensions.

In the experiment, octadecane was melted and was mixed with water in a mass fraction of $15 \%$. The mixture is mixed in an emulsion-making tank that is maintained below the crystallization temperature of octadecane. Once PCM is frozen and the slurry is formed, this slurry is then pumped to the test section by a diaphragm pump. In order to reduce the pulsation affect, a pulse dampener is placed in series in the flow path. A heat exchanger with the inlet temperature control system maintains the temperature of the 
fluid before the inlet. A section of $150 \mathrm{~mm}$ long is set as an entrance section in order to make sure the flow is fully developed. The copper tube section receives constant heat flux from the electrical heat source after the guarded heater reaches the steady state. The mixture absorbs heat from the copper wall and exits to a container where the PCM is used for recirculation.

\subsubsection{Results}

\section{Maximum effectiveness factor}

Figure 2.12 shows the effectiveness factor and performance index of the PCM suspension flow as a function of wall heat flux at two different particle concentrations $\left(\varepsilon_{p}=50 \%\right.$ and $70 \%$ ) with three different Reynolds number values of $\operatorname{Re}=10,30$, and 40 . The results are shown under the condition of $T_{f, \text { in }}=T_{p, \text { in }}=295 \mathrm{~K}\left(<T_{m, p}=301 \mathrm{~K}\right)$, $f_{p, l, i n}=0$. As shown in these figures, by keeping $L, D$ and $\varepsilon_{p}$ constant, and for a given Reynolds number, there exists an optimum wall heat flux under which the $\varepsilon_{\text {eff }}$ value is a maximum. The exact same kind of phenomena is also observed in section 2.3 for a relatively low PCM concentration and high Reynolds number suspension flow.

Compared with the pure water single-phase flow: For $\varepsilon_{p}=0.5$, the maximum heat absorbing capacity of the PCM suspension flow is about 3.3 times higher when $\operatorname{Re}=10$, i.e., the maximum effectiveness factor, $\varepsilon_{\text {eff,max }}$, equals 3.3. At $\operatorname{Re}=30, \varepsilon_{\text {eff,max }}$ is 3.6; and at $\operatorname{Re}=40, \varepsilon_{\text {eff }, \text { max }}$ is 3.7 ; as shown in Fig. 2.13. For $\operatorname{Re}=10, \varepsilon_{\text {eff, } \max }$ occurs at $q_{w}=1.6 \mathrm{~W} / \mathrm{cm}^{2}$; for $\operatorname{Re}=30$, the corresponding $q_{w}=5 \mathrm{~W} / \mathrm{cm}^{2}$; and for $\operatorname{Re}=40$, the corresponding $q_{w}=6.8 \mathrm{~W} / \mathrm{cm}^{2}$; as shown in Fig. 2.14 . 


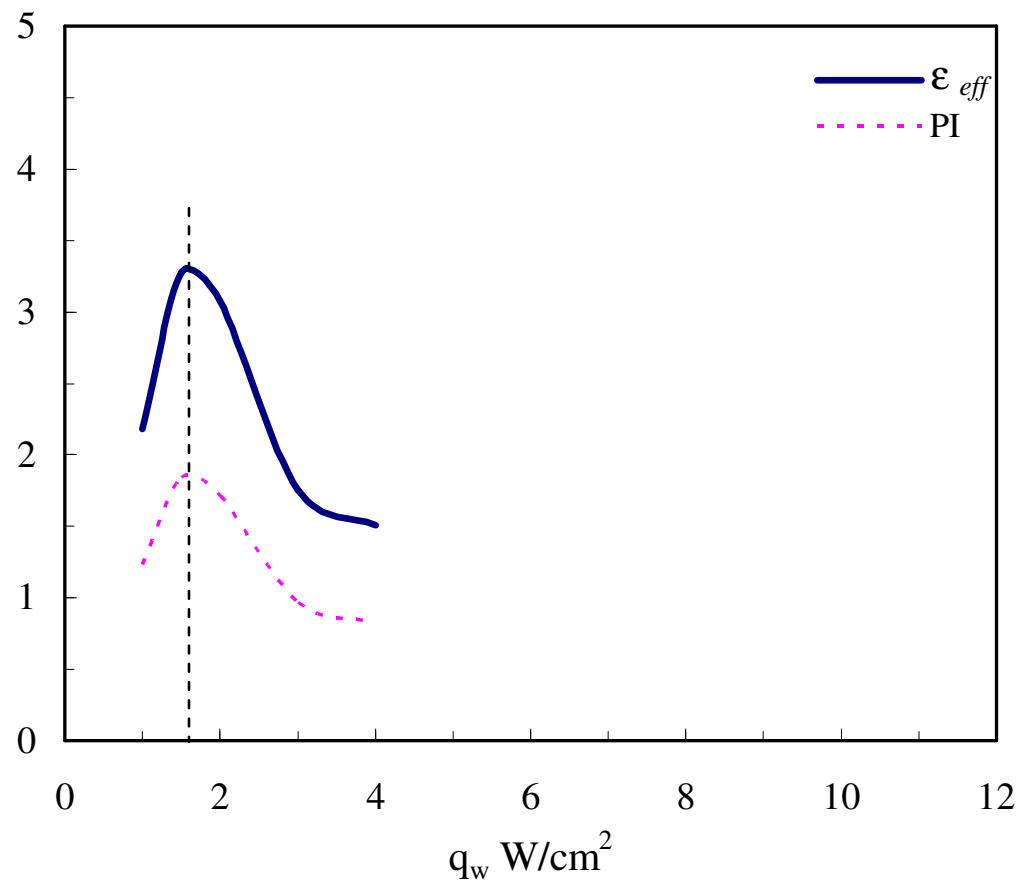

(a)

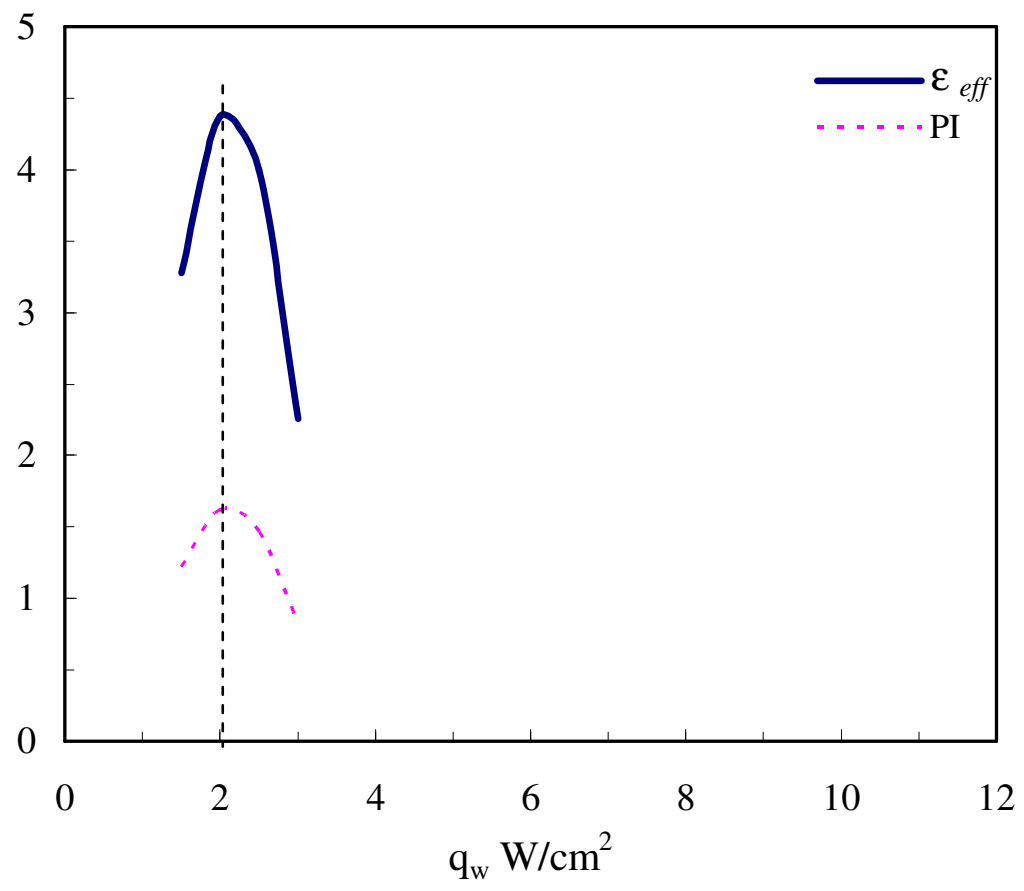

(b)

Figure 2.12 Effectiveness factor and performance index of PCM suspension flow: (a) $\varepsilon_{p}=0.5, \operatorname{Re}=10$; (b) $\varepsilon_{p}=0.7, \mathrm{Re}=10$. 


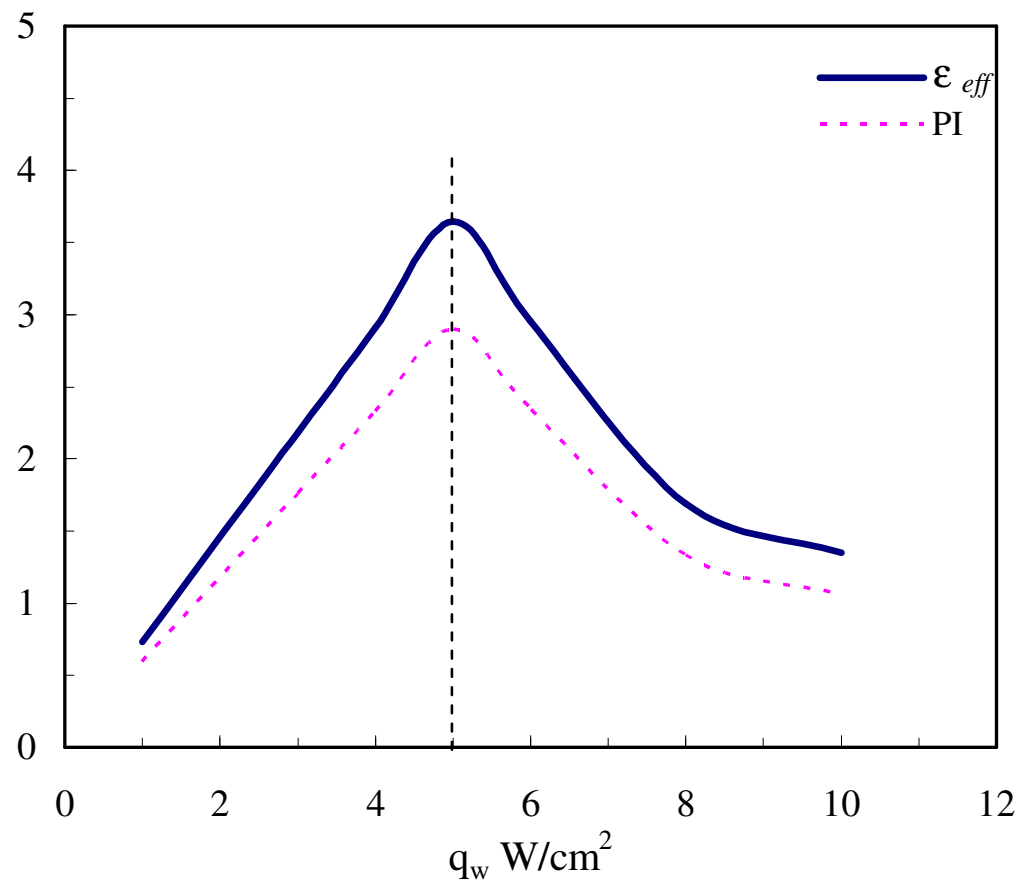

(c)

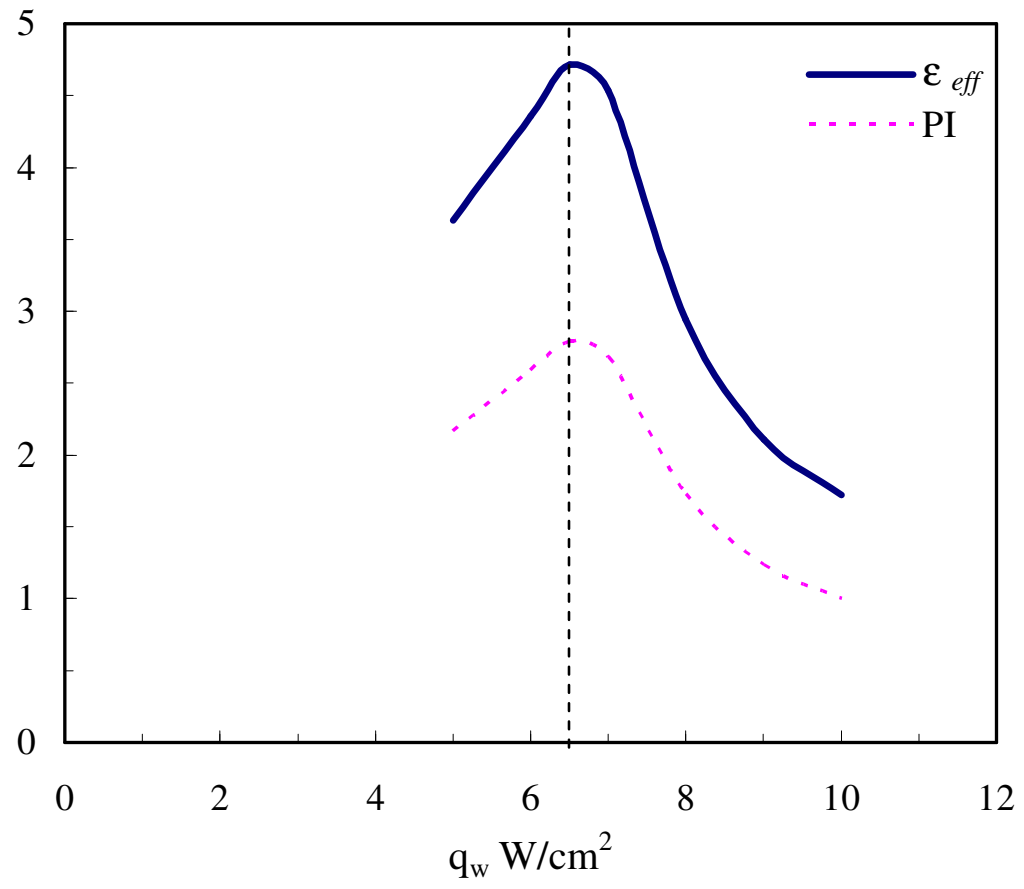

(d)

Figure 2.12 (Continued) Effectiveness factor and performance index of PCM suspension flow:

(c) $\varepsilon_{p}=0.5, \operatorname{Re}=30 ;$ (d) $\varepsilon_{p}=0.7, \operatorname{Re}=30$. 


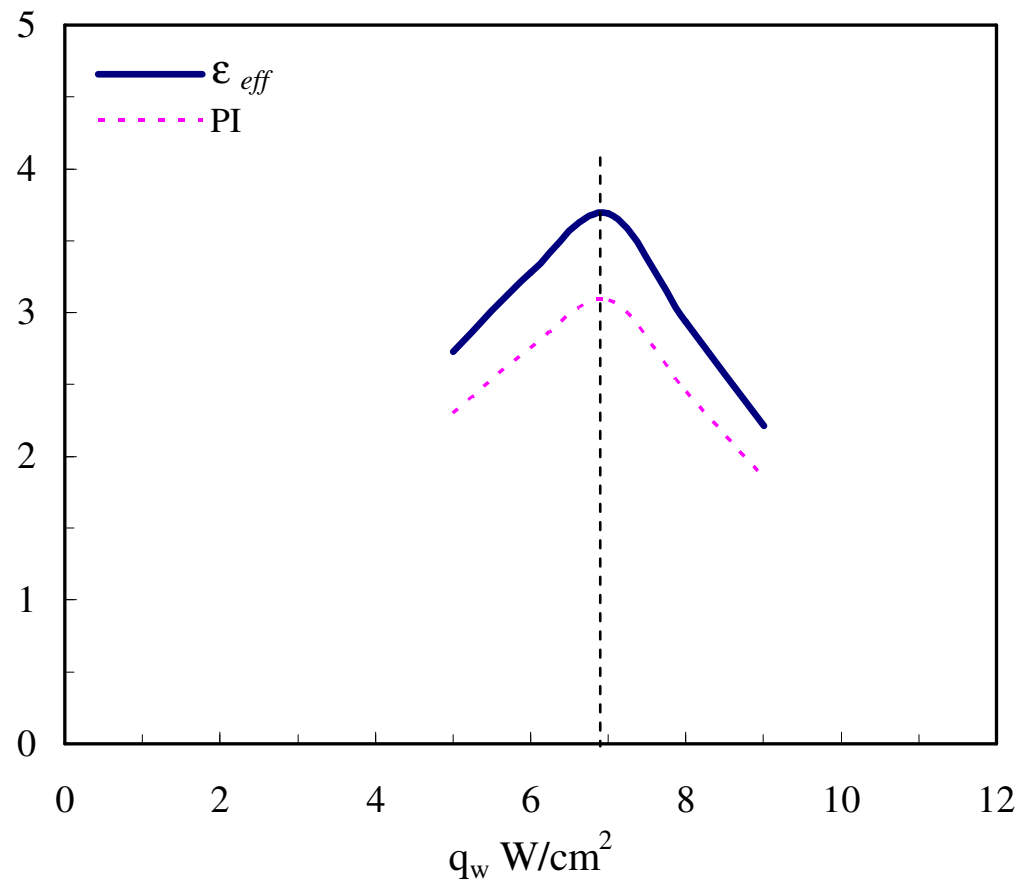

(e)

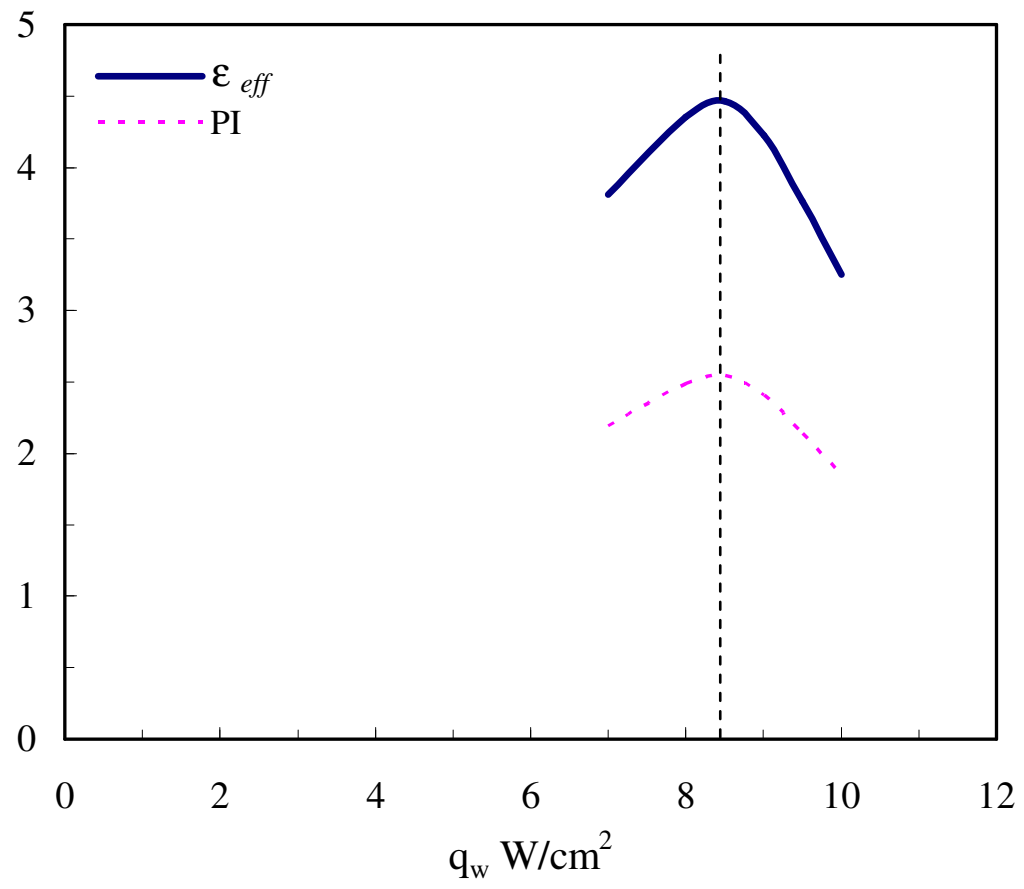

(f)

Figure 2.12 (Continued) Effectiveness factor and performance index of PCM suspension flow:

(e) $\varepsilon_{p}=0.5, \operatorname{Re}=40$; (f) $\varepsilon_{p}=0.7, \operatorname{Re}=40$. 


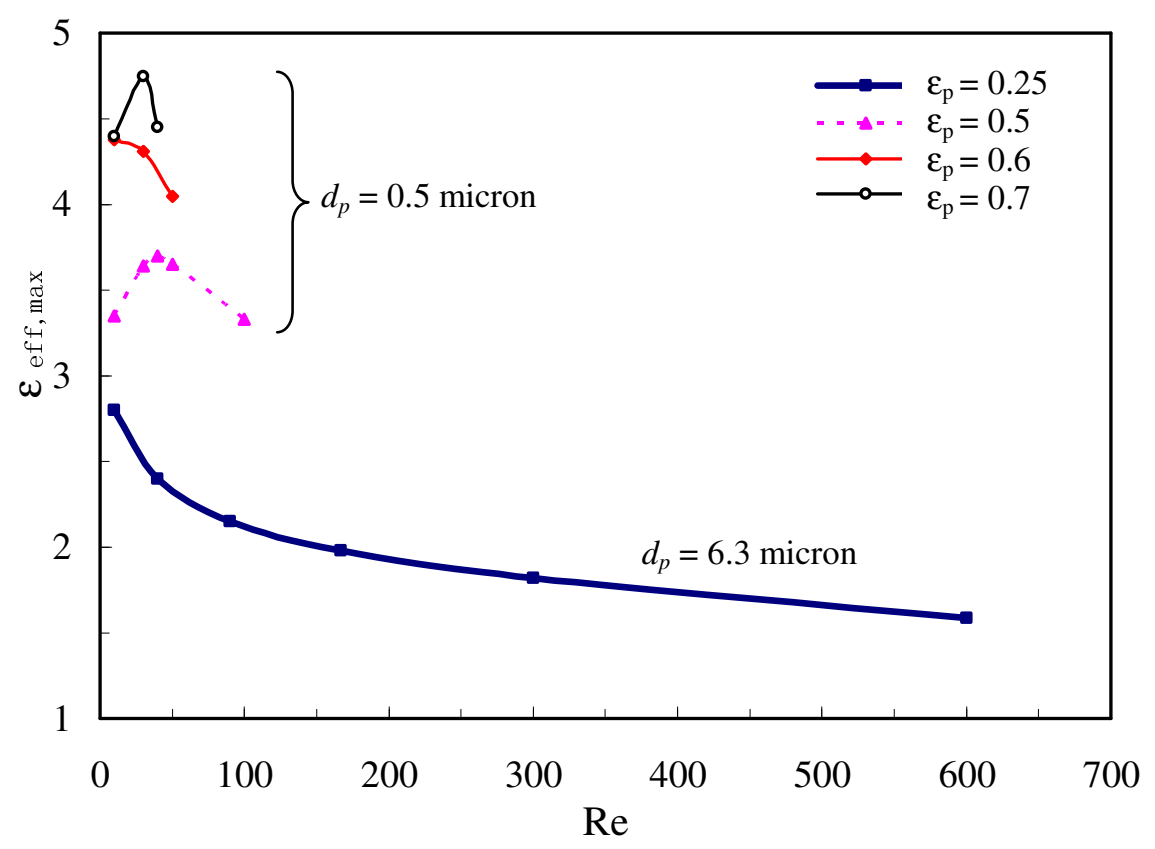

Figure 2.13 Maximum $\varepsilon_{\text {eff }}$ as a function of Re at different particle concentrations.

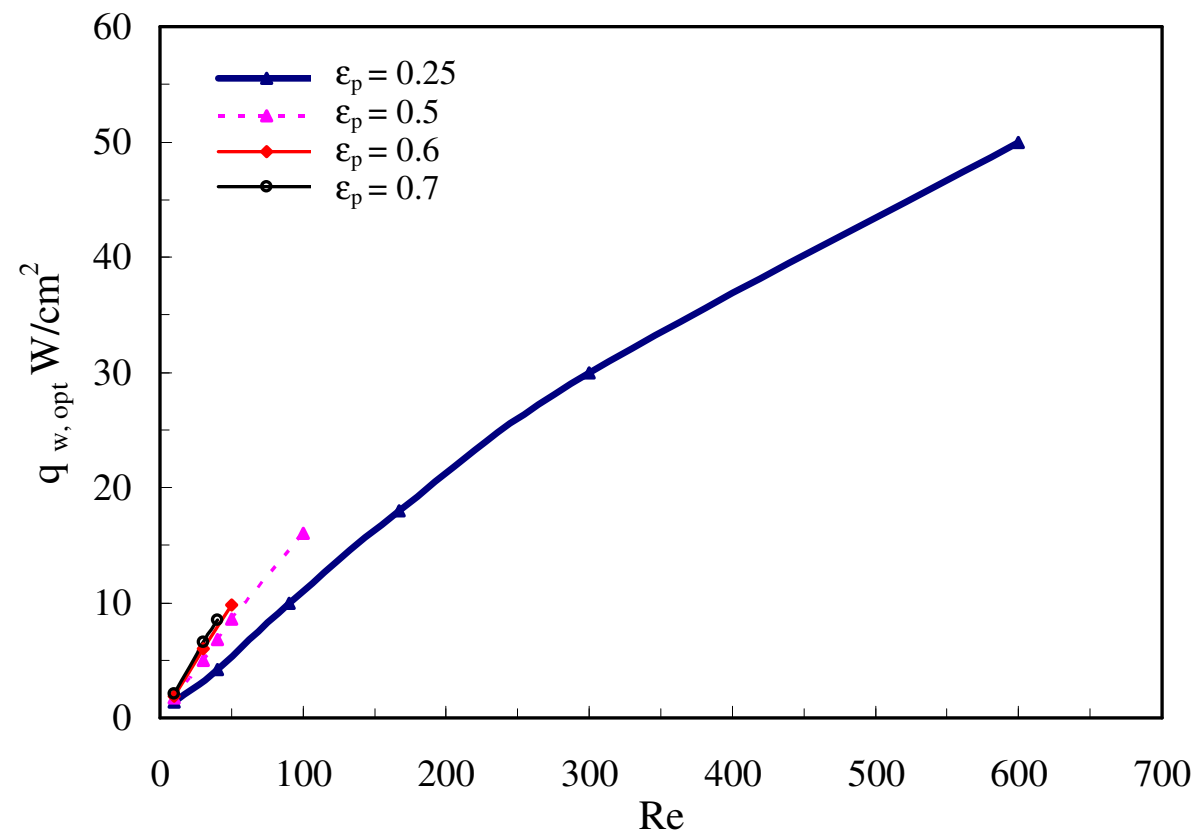

Figure 2.14 Optimal $q_{w}$ as a function of Re at different particle concentrations. 
For $\varepsilon_{p}=0.7$ : At $\operatorname{Re}=10, \varepsilon_{e f f, \text { max }}$ is 4.4 ; at $\operatorname{Re}=30, \varepsilon_{\text {eff, max }}$ is 4.7 ; and at $\operatorname{Re}=40$, $\varepsilon_{\text {eff }, \text { max }}$ is 4.5 . For $\operatorname{Re}=10, \varepsilon_{\text {eff , max }}$ occurs at $q_{w}=2.1 \mathrm{~W} / \mathrm{cm}^{2}$; for $\operatorname{Re}=30, \varepsilon_{\text {eff, max }}$ occurs at $q_{w}=6.5 \mathrm{~W} / \mathrm{cm}^{2}$; and for $\operatorname{Re}=40, \varepsilon_{\text {eff, }, \text { max }}$ occurs at $q_{w}=8.4 \mathrm{~W} / \mathrm{cm}^{2}$ (see Figs. 2.13 and 2.14). From Fig. 2.13, we can see that there exists a peak value for the $\varepsilon_{\text {eff, max }}-\operatorname{Re}$ relation at $\varepsilon_{p}=0.5$ and 0.7 ; while for $\varepsilon_{p}=0.25$ and 0.6 , we don't see this peak value for the tested Re range. Figures 2.13 and 2.14 are very useful for designing an optimal micro-channel heat exchanger.

Figure 2.12 also shows at a given $\operatorname{Re}$, when the particle concentration $\varepsilon_{p}$ increases from 0.5 to 0.7 , the corresponding maximum effectiveness factor $\varepsilon_{\text {eff, max }}$ and optimal wall heat flux $q_{w}$ also increases, which implies that for the tested Re range, the effectiveness factor can be increased by increasing the particle concentration at a given Re.

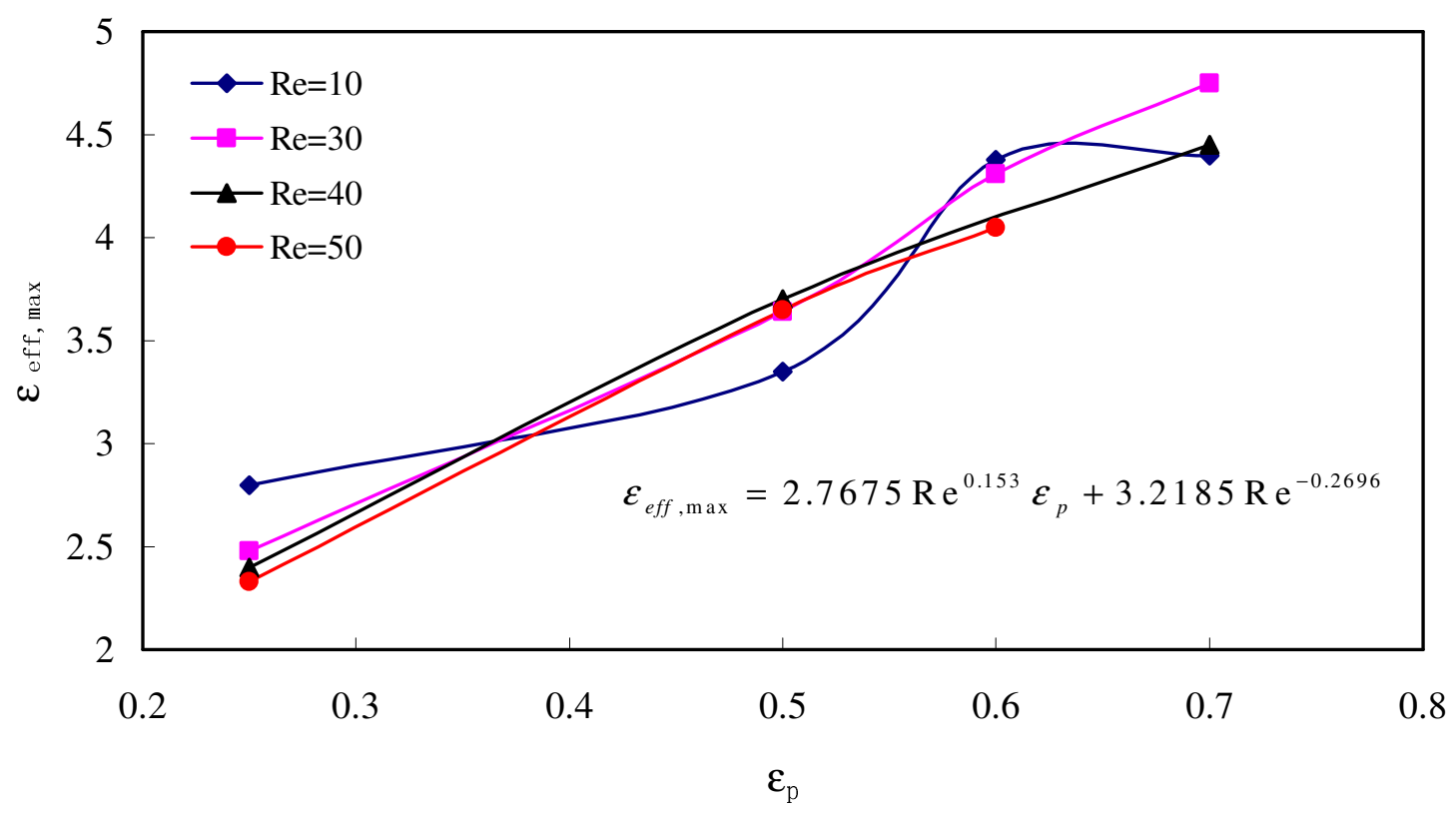

Figure 2.15 Maximum $\varepsilon_{\text {eff }}$ as a function of $\varepsilon_{p}$ at different Reynolds numbers. 
Figure 2.15 is a transform of Fig. 2.13; it shows the Maximum $\varepsilon_{\text {eff }}$ as a function of $\varepsilon_{p}$ at different Reynolds numbers. To better assist the design, a general correlation between $\varepsilon_{\text {eff,max }}, \varepsilon_{p}$, and Re is generated based on Fig. 2.15 as

$$
\varepsilon_{e f f, \max }=2.7675 \mathrm{Re}^{0.153} \varepsilon_{p}+3.2185 \mathrm{Re}^{-0.2696}
$$

Figures 2.16(a) - 2.16(d) shows the comparison between the simulation results and Eq. (2.40) under different Reynolds numbers. At $\operatorname{Re}=10$ and 30 , the simulation results lies within $20 \%$ range of Eq. (2.40). At $\operatorname{Re}=40$, the simulation results lies within $15 \%$ range. At $\operatorname{Re}=50$, the simulation results lies within $10 \%$ range. It shows for higher Reynolds numbers, the simulation results agree better with the correlation Eq. (2.40). Along with Eqs. (2.32) - (2.34), the optimal heat flux can also be determined. Eq. (2.40) can be used as a general guidance for the designer to achieve optimal design condition.

\section{Performance index}

The performance index $P I$ allows the designer to quantify and compare their new design. The results obtained in this study reveals that $P I$ can reach as high as 3.1 for $\varepsilon_{p}=0.5$, and 2.8 for $\varepsilon_{p}=0.7 . P I$ also follows the same trend as the effectiveness factor with the maximum value of $P I$ occurring at the same wall heat flux as that for $\varepsilon_{\text {eff, max }}$ (Fig. 2.12). This shows that the addition of PCM particles does not necessarily increase the pumping power required to move the flow, which is also observed in section 2.3.

\section{Particle volume fraction}

To consider the effect of particle volume fraction $\varepsilon_{p}$ on the heat transfer capacity of the PCM suspension flow, three different Reynolds numbers of $\operatorname{Re}=30,200$ and 300 


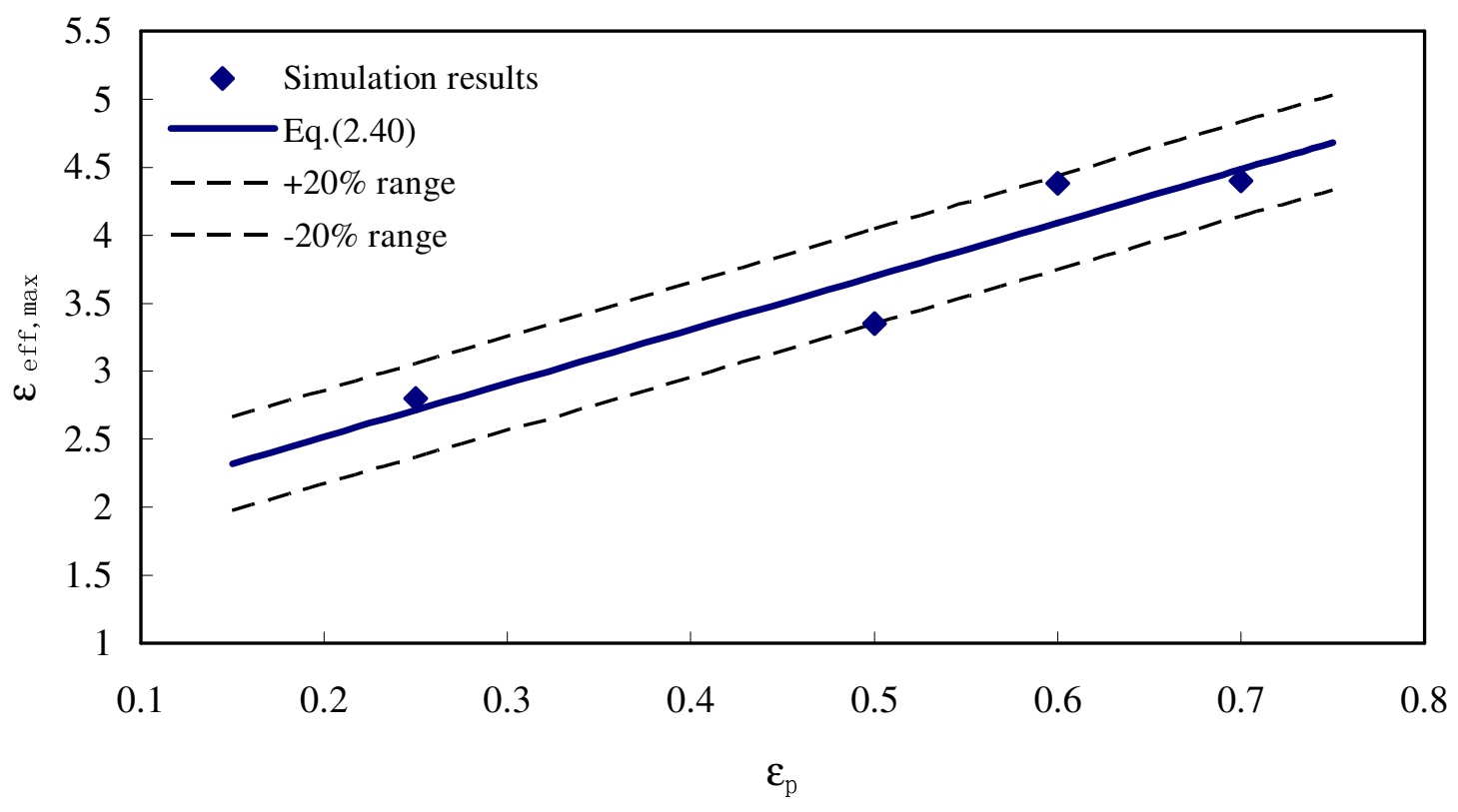

(a)

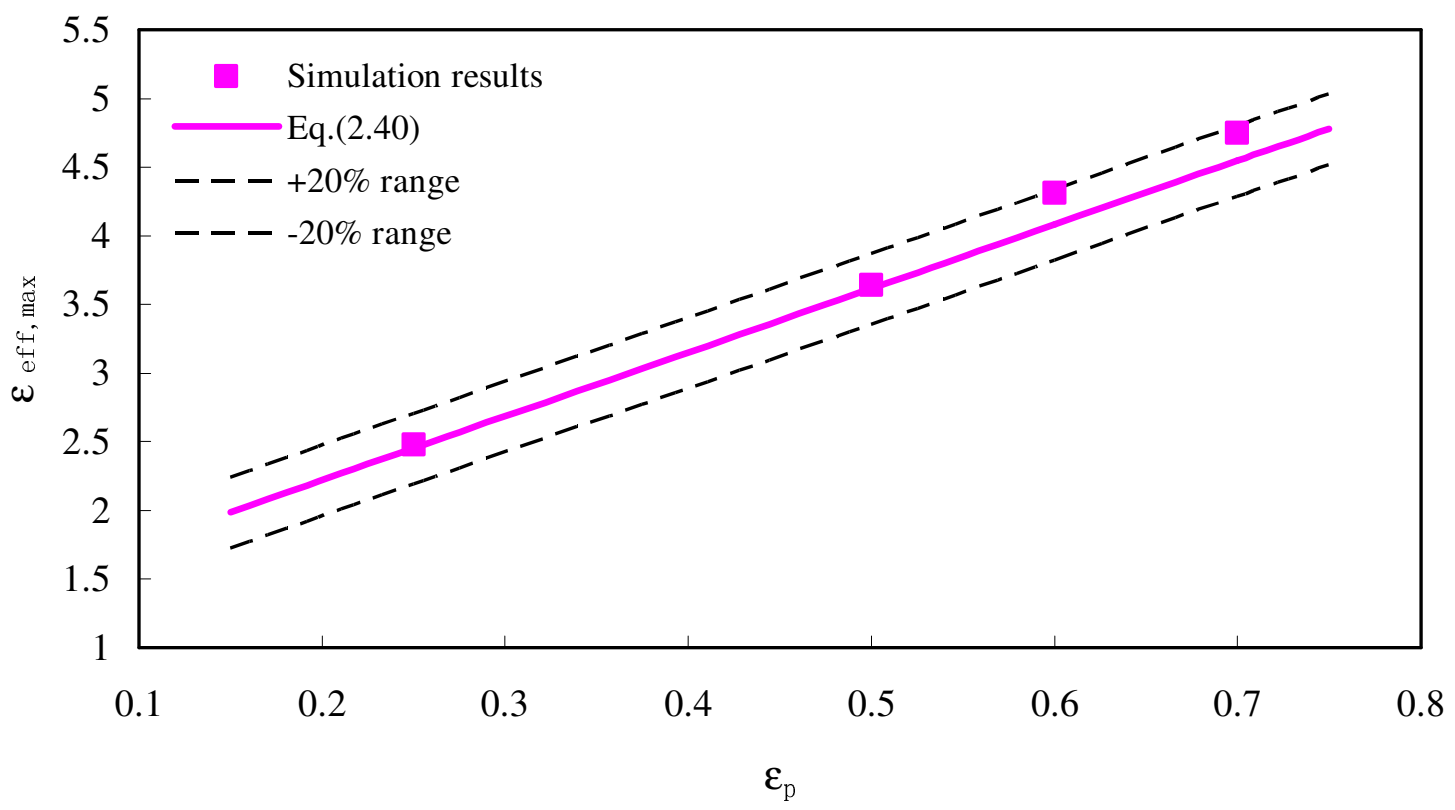

(b)

Figure 2.16 Comparison between the simulation results and the correlation:

(a) $\operatorname{Re}=10$;

(b) $\operatorname{Re}=30$. 


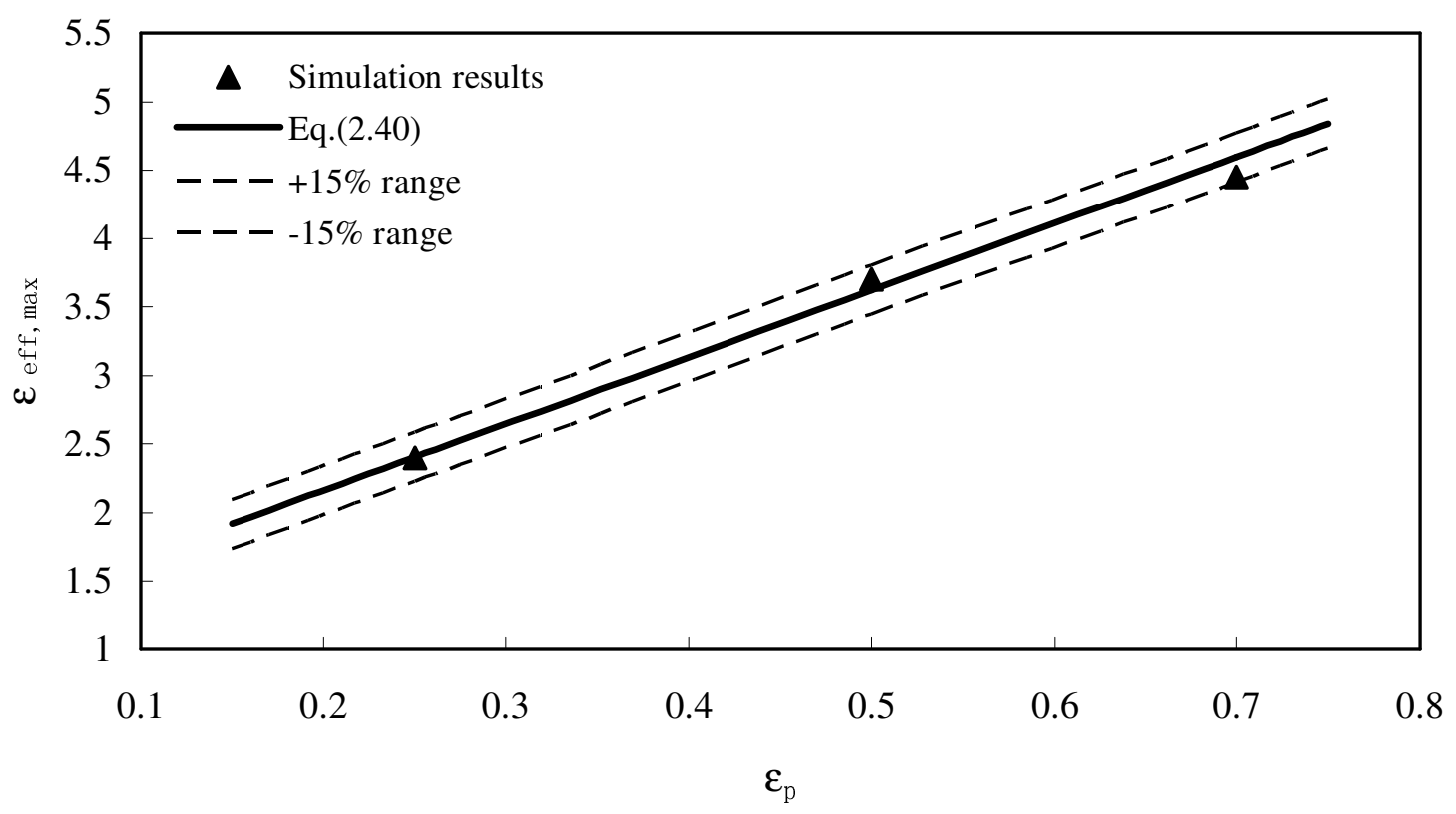

(c)

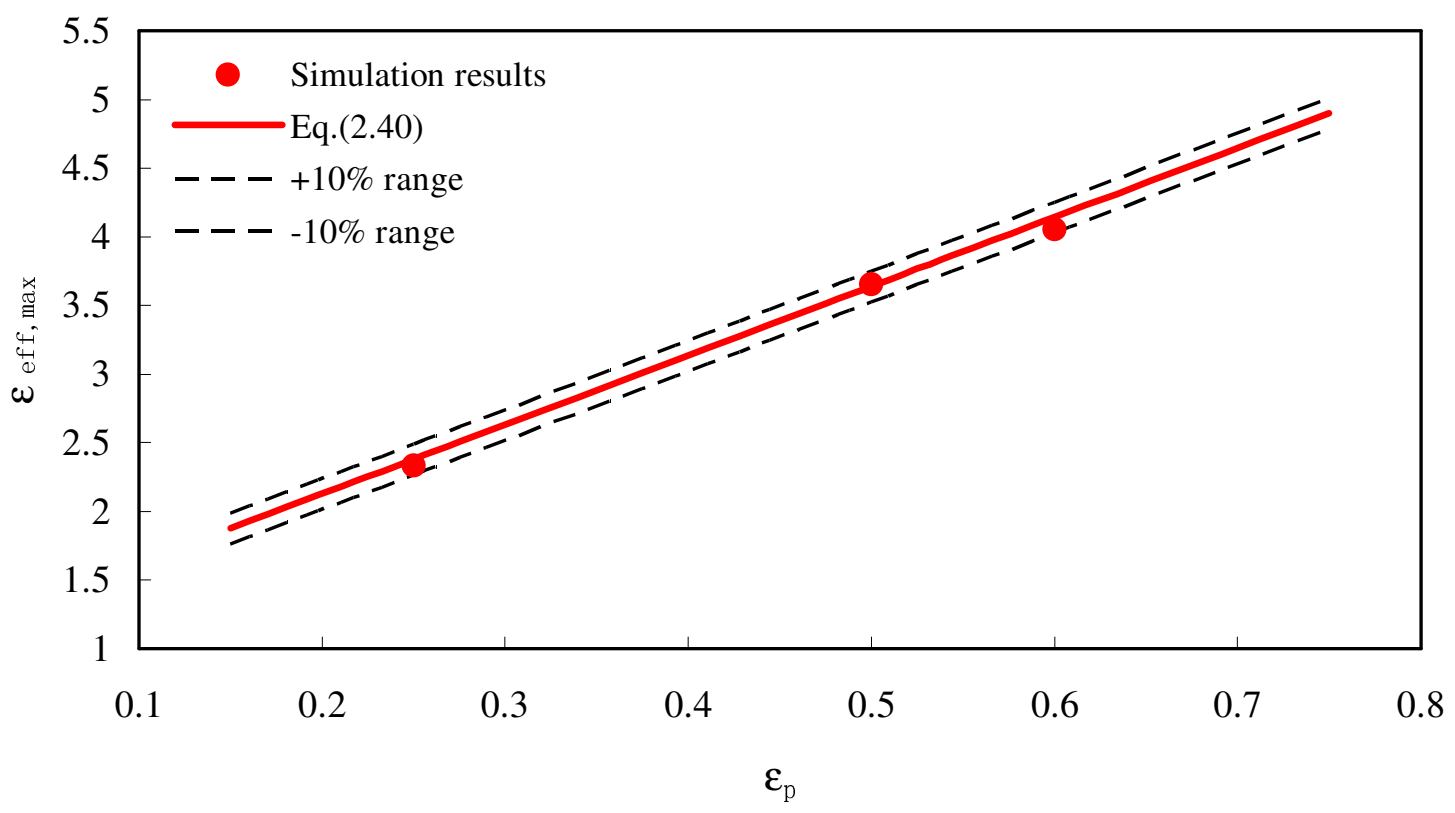

(d)

Figure 2.16 (Continued) Comparison between the simulation results and the correlation:

(c) $\operatorname{Re}=40$;

(d) $\operatorname{Re}=50$. 


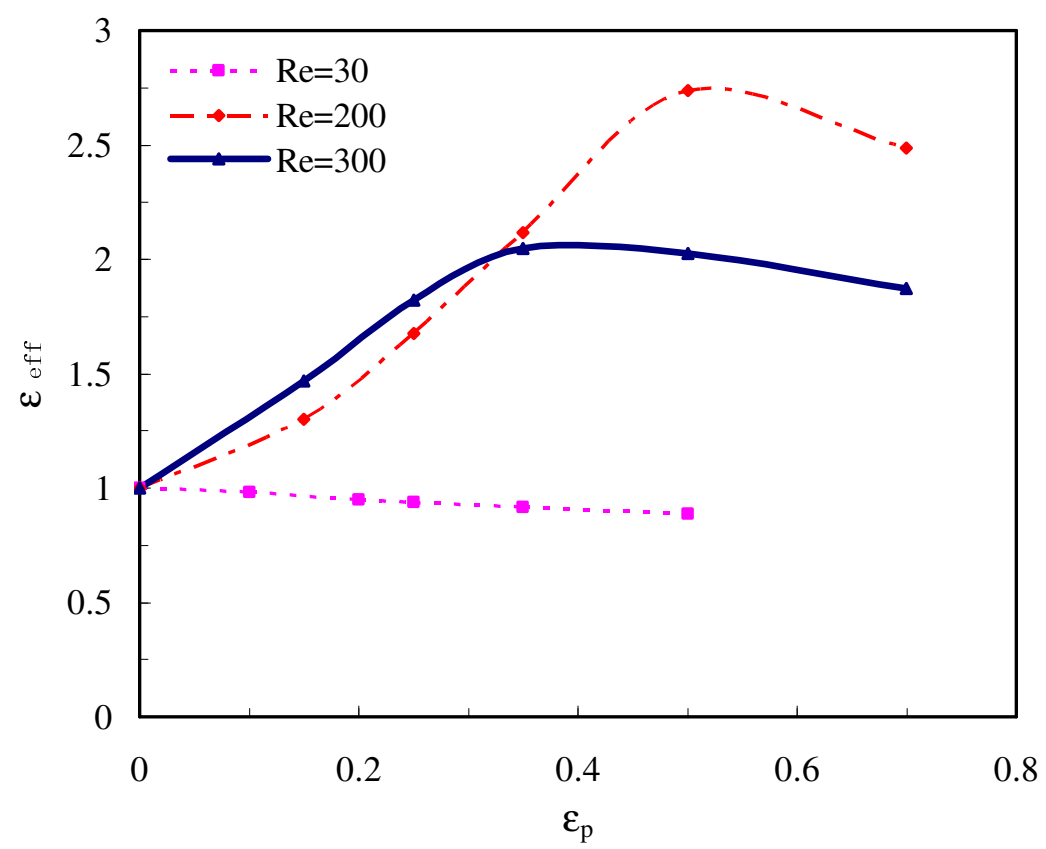

Figure 2.17 Effect of particle volume fraction on $\varepsilon_{\text {eff }}$ at different Re with $q_{w}=30 \mathrm{~W} / \mathrm{cm}^{2}$.

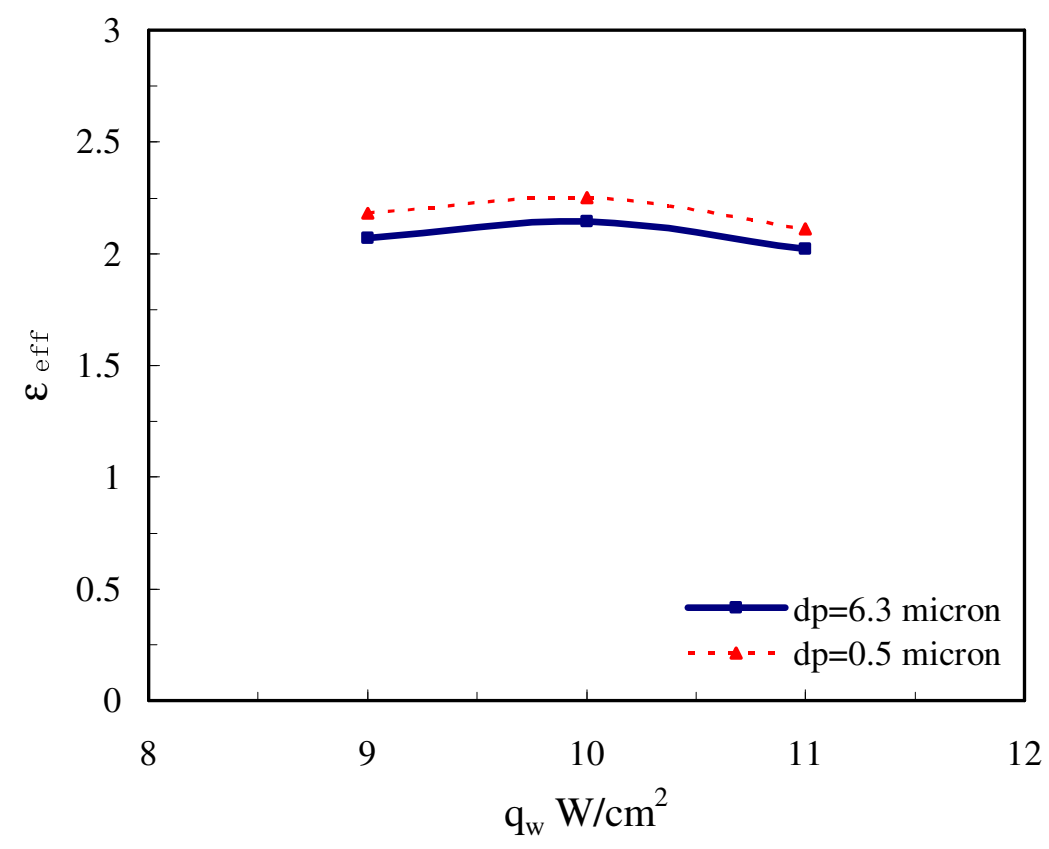

Figure 2.18 Particle size effect on $\varepsilon_{\text {eff }}$. 
are selected to see the effect at a given wall heat flux of $q_{w}=30 \mathrm{~W} / \mathrm{cm}^{2}$. Figure 2.17 shows that for $\operatorname{Re}=30, \varepsilon_{\text {eff }}$ linearly decreases with the increase of $\varepsilon_{p}$; for

$\operatorname{Re}=200$ and $300, \varepsilon_{\text {eff }}$ first increases then decreases with the increase of $\varepsilon_{p}$. The reason of this is that when there are a small number of particles, the increase of $\varepsilon_{p}$ will increase the heat absorbing capacity of the slurry, which results in an increasing $\varepsilon_{\text {eff }}$ trend; however, when $\varepsilon_{p}$ is large enough, there are too many particles in the flow, they start to become a barrier to the fluid flow, which results in a trend in decreasing the effectiveness factor.

\section{Particle size effect}

To see the influence of the particle size $d_{p}$ to the heat transfer enhancement, two different particle sizes $d_{p}=6.3$ micrometer and $d_{p}=0.5$ micrometer are selected in the simulation under the condition of $\varepsilon_{p}=0.25, \operatorname{Re}=90, T_{f, \text { in }}=T_{p, \text { in }}=295 \mathrm{~K}, f_{p, l, \text { in }}=0$. Figure 2.18 shows the $\varepsilon_{\text {eff }}-$ Re relation for these two different particle sizes. It can be seen that when $d_{p}$ decreases from 6.3 micron to 0.5 micron, $\varepsilon_{e f f, \text { max }}$ increases about $4.6 \%$. In fact, data from two different particle sizes are shown in Fig. 2.13: $d_{p}=6.3$ micrometer is used for $\varepsilon_{p}=0.25$ and $d_{p}=0.5$ micrometer is used for all the other cases. From Fig. 2.18, we can conclude that the size difference will not change our previous discussion.

\section{Experimental validation}

To validate the numerical results, experiments are conducted following the procedure discussed earlier. By using octadecane without shell materials as the PCM 


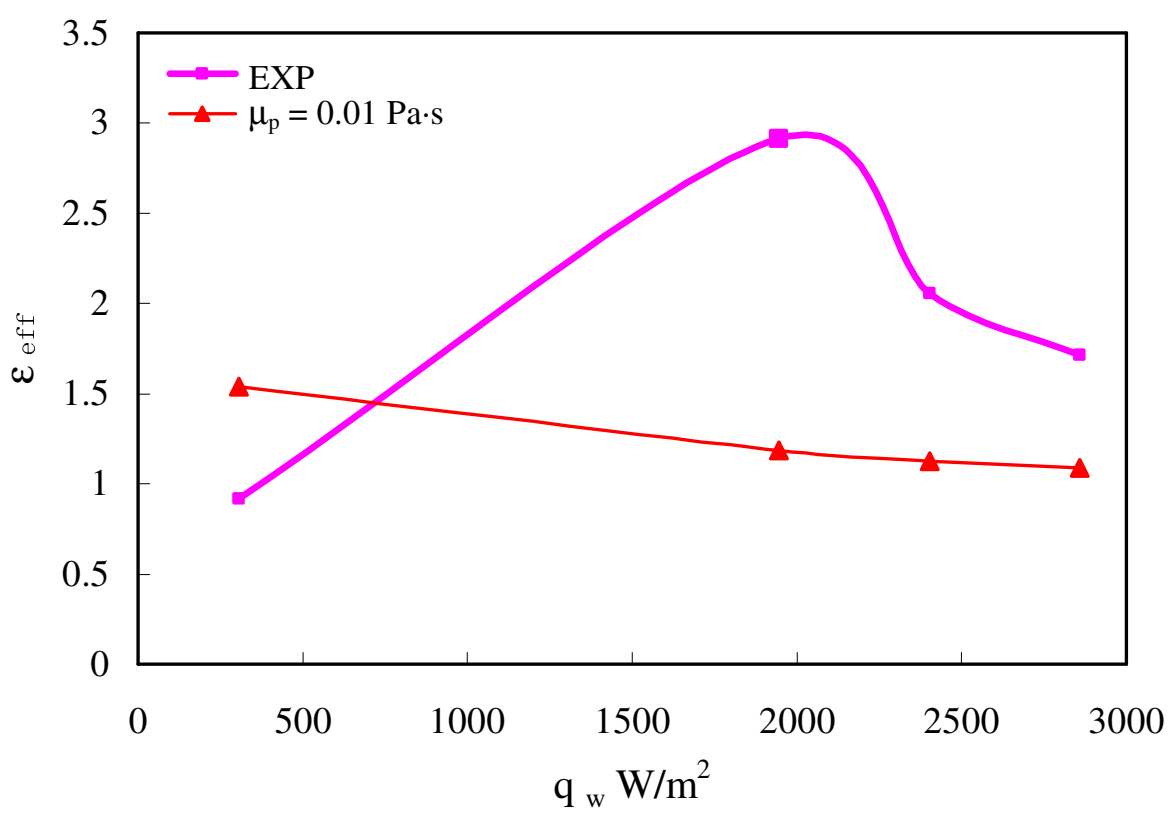

Figure 2.19 Comparison between the experiment and simulation results.

particle, at the condition of $L=0.3 \mathrm{~m}, D=0.00314 \mathrm{~m}, \varepsilon_{p}=0.15, T_{i n}=298 \mathrm{~K}$, $d_{p}=2.9 \times 10^{-5} \mathrm{~m}$, and $\mathrm{Re}=22$, four different wall heat fluxes are selected to find the optimal condition. The comparison between the experimental results and the corresponding numerical results is shown in Fig. 2.19, under the condition that the numerical simulations are conducted according to the experimentally defined boundary and geometric conditions. It can be seen that the selected first degree of approximation value of $\mu_{p}=0.01 \mathrm{~Pa} \cdot \mathrm{s}$ is not a good choice under the current experimental conditions. Another $\mu_{p}$ value that better fits the experimental results will be provided in section 2.5.

\subsubsection{Conclusions}

In this study, the numerical results for low Reynolds number limits of heat transfer enhancement due to the addition of micron-size PCM particles have been presented, 
aided with the preliminary experimental confirmation. From the above discussion, the following conclusion may be drawn:

1. The current numerical model works reasonably well for low $\operatorname{Re}$ and high $\varepsilon_{p}$ applications and is capable of predicting the optimal conditions for heat transfer enhancement.

2. For a given Reynolds number and particle volume fraction, there exists an optimal wall heat flux that will yield a maximum effectiveness factor $\varepsilon_{\text {eff, max }}$; there also exists a maximum performance index $P I_{\max }$ at the optimal wall heat flux. This indicates that at the optimal condition, PCM flow not only can significanly enhance heat transfer, but also becomes more efficient. The physcial condition for this to happen is to ensure the phase change happens within the significant (majority) portion of the heat transfer region.

3. The $\varepsilon_{\text {eff,max }}-$ Re relation has a peak vaue for $\varepsilon_{p}=0.5$ and 0.7 , which means that to achieve the best heat transfer enhancement effect with the PCM flow, one needs to find a unique match between $\operatorname{Re}$ and $\varepsilon_{p}$. Increasing the Reynolds number does not always enhance the heat transfer for PCM flow.

4. The correlation between $\varepsilon_{e f f, \max }, \varepsilon_{p}$, and Re can give a general guidance for the designer to achieve optimal design condition.

5. A decrease in particle sizes tends to enhance heat transfer.

6. The experimental results prove the existence of the optimal condition as predicted by the numerical simulation, and could be reasonablly extrapolated from microchannel to minichannel applications where the continuum flow assumption holds. 
The above conclusions will assist designers in making their decisions when selecting a micro-pump suitable for low Re micro or mini scale heat transfer devices.

\subsection{Slurry Viscosity Study and Its Influence on the Heat Transfer Enhancement Effect of PCM Slurry Flow}

The two-phase non-thermal-equilibrium based model used in our simulation is based on the continuum theory. It introduces a particle viscosity, $\mu_{p}$, which is not available for the system of liquid-solid two-phase flow. A first degree of approximation value of $\mu_{p}=0.01 \mathrm{~Pa} \cdot \mathrm{s}$ is used in the previous study, but its influence to the simulation results remains unknown. Therefore, this section will focus on the study of the particle viscosity $\mu_{p}$ and its relation to the bulk viscosity $\mu_{b}$ to clarify its influence to the heat transfer enhancement effect of the PCM slurry flow.

\subsubsection{Review of suspension viscosity}

Suspensions play an important role in many established industries, including mineral processing, paper manufacture, manufacture of solid fuel rocket propellants and oil exploration. The bulk viscosity of suspensions has been widely studied in the last century. Different correlations, theoretical or empirical, have been developed to calculate the dependence of bulk viscosity $\mu_{b}$ on the particle concentration $\mathcal{E}_{p}$. In this paper, we limited ourselves mostly to the discussion of the suspensions of rigid spherical particles.

\section{Theoretical viscosity study for suspension flow}

A list of theoretical correlations and equations is presented in Table 2.4. The first attempt to treat theoretically the bulk viscosity of suspensions is due to Einstein (1906 and 1911) [T1 in Table 2.4]. The derivation introduces certain simplifications which are 
valid only for infinitely dilute suspensions. Following Einstein's work, great efforts have been made to model the viscosity of concentrated suspensions theoretically. To extend Einstein's equation to higher concentration, the interactions between particles must be considered.

For mono-disperse rigid spheres suspensions, Guth and Simha (1936) [T2] extended the Einstein equation by assuming continuity of velocity condition at the particle surface. Their formula agrees with measurements about up to the concentration of the order of 8\%. Vand $\left(1948^{1}\right)$ [T3 and T4] independently derived another formula by considering the interactions and collisions between particles. Then, a power series formula is derived for rigid, non-solvated spheres without mutual forces and without Brownian motion and is valid over a wide range of concentration. Robinson (1949) [T5] extended the Einstein equation to higher concentration by considering that the specific viscosity of the suspension is not only proportional to the volume concentration, but also inversely proportional to the volume of free liquid in the suspension. Although the equation is valid for suspensions of inert spheres at any concentration, the extension of it to suspensions of practical significance is complicated because of the relative sediment volume $S^{\prime}$, which depends on the rate of shear and the particle size and will not be a constant for any given suspension. Another extension of the Einstein equation is done by Mooney (1951) [T6], who described the interaction between particles as a crowding effect. By comparing with existing experimental data, Mooney claimed that his formula is valid for a concentration range from 0 to $50 \%$. Krieger and Dougherty (1959) [T7] derived a formula valid over the entire concentration range and have an excellent fit to their own experimental data. The Frankel-Acrivos (1967) [T8] model was derived under the assumption that the 
Table 2.4 Theoretical viscosity correlations for suspension flow

\begin{tabular}{|c|c|c|c|}
\hline No. & $\begin{array}{c}\text { Equation } \\
\text { (or Equation Set) }\end{array}$ & Operational Conditions & Reference \\
\hline $\mathrm{T} 1$ & $\mu_{b}=\mu_{f}\left(1+k \varepsilon_{p}\right)$ & $\begin{array}{l}\text { valid only for infinitely diluted suspensions } \\
\text { of rigid spheres; }\end{array}$ & $\begin{array}{c}\text { Einstein } \\
(1906 \text { and 1911) }\end{array}$ \\
\hline $\mathrm{T} 2$ & $\mu_{b}=\mu_{f} \frac{1+0.5 \varepsilon_{p}-0.5 \varepsilon_{p}^{2}}{1-2 \varepsilon_{p}-9.6 \varepsilon_{p}^{2}}$ & $\begin{array}{l}\varepsilon_{p} \leq 8 \% \text {; } \\
\text { valid for suspension of rigid spheres with } \\
\text { the assumption of continuity of velocity } \\
\text { condition at the particle surface; }\end{array}$ & $\begin{array}{l}\text { Guth and Simha } \\
\text { (1936) }\end{array}$ \\
\hline $\mathrm{T} 3$ & $\mu_{b}=\mu_{f} e^{\frac{k \varepsilon_{p}+r_{2}\left(k_{2}-k\right) \varepsilon_{p}^{2}+\cdots}{1-Q \varepsilon_{p}}}$ & $\begin{array}{l}\text { valid for suspension of rigid spherical } \\
\text { particles by considering the mutual } \\
\text { interactions and collisions between } \\
\text { particles; } k_{2} \text { is the shape factor of collision } \\
\text { doublets; } r_{2} \text { is the collision time constant; } Q \\
\text { is the hydrodynamics interaction constant; }\end{array}$ & $\begin{array}{l}\text { Vand } \\
\left(1948^{1}\right)\end{array}$ \\
\hline $\mathrm{T} 4$ & $\mu_{b}=\mu_{f}\left(1+2.5 \varepsilon_{p}+7.349 \varepsilon_{p}^{2}+\cdots\right)$ & $\begin{array}{l}\text { valid over a wide range of concentration for } \\
\text { non-solvated rigid spheres without mutual } \\
\text { forces and Brownian motion; }\end{array}$ & $\begin{array}{l}\text { Vand } \\
\left(1948^{1}\right)\end{array}$ \\
\hline T5 & $\mu_{b}=\mu_{f}\left(1+\frac{k \varepsilon_{p}}{1-S \varepsilon_{p}}\right)$ & $\begin{array}{l}\text { valid for all concentrations; } \\
S \text { is the relative sediment volume; }\end{array}$ & $\begin{array}{l}\text { Robinson } \\
\text { (1949) }\end{array}$ \\
\hline $\mathrm{T} 6$ & $\begin{array}{l}\mu_{b}=\mu_{f} e^{\frac{2.5 \varepsilon_{p}}{1-k^{\prime} \varepsilon_{p}}} \\
1.35<k^{\prime}<1.91\end{array}$ & $\begin{array}{l}\text { experimentally validated range is: } \varepsilon_{p} \leq 50 \% \text {; } \\
\text { valid for suspension of rigid spheres; } \\
k \text { ' is the self-crowding factor; }\end{array}$ & $\begin{array}{l}\text { Mooney } \\
(1951)\end{array}$ \\
\hline $\mathrm{T} 7$ & $\mu_{b}=\mu_{f}\left(1-\frac{\varepsilon_{p}}{\varepsilon_{m}}\right)^{-2.5 \varepsilon_{m}}$ & $\begin{array}{l}\text { valid for all concentrations for mono- } \\
\text { disperse suspension of rigid spheres; }\end{array}$ & $\begin{array}{l}\text { Krieger and } \\
\text { Dougherty } \\
(1959)\end{array}$ \\
\hline $\mathrm{T} 8$ & $\mu_{b}=\mu_{f} \frac{9}{8} \frac{\left(\varepsilon_{p} / \varepsilon_{m}\right)^{1 / 3}}{\left[1-\left(\varepsilon_{p} / \varepsilon_{m}\right)^{1 / 3}\right]}$ & $\begin{array}{l}\text { only valid for } \varepsilon>20 \% \text {; } \\
\text { for mono-disperse suspension of rigid } \\
\text { spheres with the assumption that the } \\
\text { hydrodynamic interactions between nearest } \\
\text { neighboring particles dominate the } \\
\text { suspension flow; }\end{array}$ & $\begin{array}{l}\text { Frankel and Acrivos } \\
\text { (1967) }\end{array}$ \\
\hline T9 & $\mu_{b}=\mu_{f}\left(1-\frac{\varepsilon_{p}}{0.57}\right)^{-1.50}$ & $\begin{array}{l}\text { valid for all concentrations for suspension of } \\
\text { rigid spheres with low shear rate; }\end{array}$ & $\begin{array}{r}\text { Krieger } \\
(1972)\end{array}$ \\
\hline $\mathrm{T} 10$ & $\mu_{b}=\mu_{f}\left\{\frac{9}{4}\left[1+\frac{h}{2 r}\right]^{-1} \times\left[\frac{1}{h / r}-\frac{1}{1+h / r}-\frac{1}{(1+h / r)^{2}}\right]+\left(1+\frac{5}{2} \varepsilon_{p}\right)\right\}$ & $\begin{array}{l}\text { valid for all concentrations for suspension of } \\
\text { uniform, solid, neutrally buoyant spheres } \\
\text { with hydrodynamic forces only; } \\
r \text { is the sphere radius and } h \text { is the minimum } \\
\text { separation distance between two particles; }\end{array}$ & $\begin{array}{c}\text { Graham } \\
(1981)\end{array}$ \\
\hline $\mathrm{T} 11$ & $\begin{array}{l}\mu_{b}=\mu_{f}\left(1-\frac{\varepsilon_{p}}{\varepsilon_{m}}\right)^{-2}\left[1-0.4 \frac{\varepsilon_{p}}{\varepsilon_{m}}+0.34\left(\frac{\varepsilon_{p}}{\varepsilon_{m}}\right)^{2}\right] \\
\varepsilon_{m}=0.64\end{array}$ & $\begin{array}{l}\text { valid for all concentrations for suspension of } \\
\text { uniform sized spheres; }\end{array}$ & $\begin{array}{l}\text { Bicerano et al. } \\
\text { (1999) }\end{array}$ \\
\hline $\mathrm{T} 12$ & $\mu_{b}=\mu_{f}\left(1-\varepsilon_{p}\right)^{-2.5}$ & $\begin{array}{l}\text { valid for all concentrations for rigid spheres } \\
\text { of very diverse size; }\end{array}$ & $\begin{array}{l}\text { Roscoe } \\
(1952)\end{array}$ \\
\hline $\mathrm{T} 13$ & $\mu_{b}=\mu_{f}\left(1-1.35 \varepsilon_{p}\right)^{-2.5}$ & $\begin{array}{l}\text { valid for high concentration for rigid } \\
\text { spheres of equal size; }\end{array}$ & $\begin{array}{l}\text { Roscoe } \\
(1952)\end{array}$ \\
\hline T14 & $\begin{array}{l}\mu_{b}=\mu_{f} f\left(\varepsilon_{p}\right) \\
f\left(\varepsilon_{p}\right)=\frac{1+1.5 \varepsilon_{p}}{1-\varepsilon_{p}-0.8946 \varepsilon_{p}^{2}}\end{array}$ & $\begin{array}{l}\text { valid for all concentrations for suspension of } \\
\text { mono-disperse rigid spheres; }\end{array}$ & $\begin{array}{l}\text { Kovar and Fortelny } \\
\text { (1984) }\end{array}$ \\
\hline $\mathrm{T} 15$ & $\begin{array}{l}\mu_{b}=\mu_{f} f\left[\frac{\left(1-c^{\prime}\right) \varepsilon_{p}}{1-c^{\prime} \varepsilon_{p}}\right] f\left(c^{\prime} \varepsilon_{p}\right) \\
f\left(\varepsilon_{p}\right)=\frac{1+1.5 \varepsilon_{p}}{1-\varepsilon_{p}-0.8946 \varepsilon_{p}^{2}} \quad c^{\prime}=\frac{V_{2}}{V_{1}+V_{2}}\end{array}$ & $\begin{array}{l}\text { valid for all concentrations for suspension of } \\
\text { bi-disperse rigid spheres with } r_{2} \geqslant r_{l} \text {; } \\
V_{l} \text { and } V_{2} \text { are the volumes of the first and } \\
\text { second type spheres; }\end{array}$ & $\begin{array}{l}\text { Kovar and Fortelny } \\
\text { (1984) }\end{array}$ \\
\hline
\end{tabular}


hydrodynamic interactions between nearest neighboring particles dominate the flow. So it is valid only for high concentration over $20 \%$. Krieger's (1972) [T9] low shear rate model spans the whole range of concentration. Graham (1981) [T10] derived a formula by using a cell theory approach. His formula is valid over the entire concentration range for suspension of uniform rigid spheres with hydrodynamic forces only. Bicerano et al. (1999) [T11] also suggested an equation for suspension of uniform sized spheres.

There are also some discussions about non-uniform particle size suspensions. Roscoe (1952) [T12 and T13] discussed the dependence of suspension viscosity on the particle size distribution. He found that the Einstein equation is valid up to concentration of $5 \%$ for uniform size spheres. He also derived an equation for spheres of very diverse size and valid for all concentrations. Another formula he derived is for equal size spheres and valid at high concentrations only. Kovar and Fortelny (1984) [T14 and T15] derived two equations, one is for mono-disperse rigid spheres, the other one is for bi-disperse rigid spheres with considerably different diameters. Both equations are valid for all concentration range.

\section{Experimental viscosity study for suspension flow}

Except theoretical correlations, a lot of empirical or semi-empirical correlations have also been proposed. A list of empirical correlations is presented in Table 2.5.

Arrhenius (1887) [E1 in Table 2.5] is the first one who found an empirical correlation for suspension viscosity, which is valid for all concentration with the assumption of no interaction between particles. Eilers (1941) [E2] represented his measurements by a formula, which in the format similar to the theoretical correlation obtained by Robinson (1949) [T5]. Vand (1945) [E3] derived a formula from Einstein's 
equation by considering the interaction between particles and experimentally determined the coefficient of the formula. His formula is valid up to concentration of $37 \%$ for uniform rigid spheres. After derived a theoretical correlation (Vand, 1948 ${ }^{1}$, [T3 and T4]), Vand $\left(1948^{2}\right)$ [E4] constructed his own experiment and got a correlation in the same format as his theoretical formula but with different coefficients. This empirical correlation agrees very well with his theoretical formula. Higginbotham et al. (1958) [E5] suggested a modified form of the Einstein equation and corrected it through experiment by considering the wall effect of the used capillary-tube viscometer. Rutgers (1962) [E6] gave a thorough review to the available experimental data and generated an averaged curve. A formula for this average curve is generated in this paper by using the data provided in (Rutgers, 1962). According to Rutgers, this average curve is valid up to concentration of 50\%. Thomas (1965) [E7] corrected the data of the sixteen experiments that he considered to be the most careful and generated a formula valid for all concentration range. Chong et al. (1971) [E8] correlated their experimental data and generated a formula valid for bi-disperse spheres. Kitano et al. (1981) [E9] proposed a very simple correlation and was considered the best empirical correlation by Metzner (1985). Sengun and Probstein (1989) [E10] proposed a semi-empirical formula which is appropriate only for high concentrations $\left(\varepsilon_{p}>20 \%\right)$ and is not a valid expression for dilute suspensions. Storms et al. (1990) [E11] proposed a similar correlation to (Chong et al., 1971) for bi-disperse spheres suspensions. Ilic and Phan-Thien (1994) [E12] used the falling needle technique to measure the suspension viscosity. A formula is generated in this paper by using Ilic and Phan-Thien's data. He et al. (2001) [E13] developed a 
Table 2.5 Empirical viscosity correlations for suspension flow

\begin{tabular}{|c|c|c|c|}
\hline No. & $\begin{array}{c}\text { Equation } \\
\text { (or Equation Set) }\end{array}$ & Operational Conditions & Reference \\
\hline E1 & $\mu_{b}=\mu_{f} e^{k \varepsilon_{p}}$ & $\begin{array}{l}\text { valid for all concentrations with the } \\
\text { assumption of no interaction between } \\
\text { particles; } k=2.5 \text { for spherical particles; }\end{array}$ & $\begin{array}{l}\text { Arrhenius } \\
\text { (1917) }\end{array}$ \\
\hline E2 & $\mu_{b}=\mu_{f}\left[1+\frac{1.25 \varepsilon_{p}}{1-\left(\varepsilon_{p} / 0.78\right)}\right]^{2}$ & $\begin{array}{l}\text { valid for all concentrations for } \\
\text { suspension of rigid spheres; }\end{array}$ & $\begin{array}{l}\text { Eilers } \\
(1941)\end{array}$ \\
\hline E3 & $\mu_{b}=\mu_{f}\left(1-\varepsilon_{p}-1.16 \varepsilon_{p}^{2}\right)^{-2.5}$ & $\begin{array}{l}\varepsilon_{p} \leq 37 \% \\
\text { valid for suspension of rigid spheres; }\end{array}$ & $\begin{array}{l}\text { Vand } \\
(1945)\end{array}$ \\
\hline $\mathrm{E} 4$ & $\mu_{b}=\mu_{f}\left(1+2.5 \varepsilon_{p}+7.17 \varepsilon_{p}^{2}+16.2 \varepsilon_{p}^{3}+\cdots\right)$ & $\begin{array}{l}\text { experimentally validated range is } \\
\varepsilon_{p} \leq 50 \% ; \\
\text { valid for suspension of rigid spheres; }\end{array}$ & $\begin{array}{c}\text { Vand } \\
\left(1948^{2}\right)\end{array}$ \\
\hline E5 & $\begin{array}{l}\mu_{b}=\mu_{f}\left(1-K \varepsilon_{p}\right)^{-1} \\
2.33<K<2.46\end{array}$ & $\begin{array}{l}\varepsilon_{p}<28 \% \text {; } \\
\text { valid for suspension of rigid spheres } \\
\text { with correction for wall effects of the } \\
\text { viscometer; } K \text { is a constant; }\end{array}$ & $\begin{array}{l}\text { Higginbotham et al. } \\
\text { (1958) }\end{array}$ \\
\hline E6 & $\mu_{b}=\mu_{f}\left(\begin{array}{l}1.0005+0.2648 \varepsilon_{p} \\
+102.95 \varepsilon_{p}^{2}-1236.5 \varepsilon_{p}^{3} \\
+7478.5 \varepsilon_{p}^{4}-20419 \varepsilon_{p}^{5} \\
+21778 \varepsilon_{p}^{6}\end{array}\right)$ & $\begin{array}{l}\varepsilon_{p}<50 \% \text {; } \\
\text { valid for suspension of rigid spheres in } \\
\text { Newtonian fluid; } \\
\text { the correlation is generated from } \\
\text { experimental data; }\end{array}$ & $\begin{array}{l}\text { Rutgers } \\
(1962)\end{array}$ \\
\hline E7 & $\mu_{b}=\mu_{f}\left[\begin{array}{l}1+2.5 \varepsilon_{p}+10.05 \varepsilon_{p}^{2} \\
+0.00273 e^{16.6 \varepsilon_{p}}\end{array}\right]$ & $\begin{array}{l}\text { valid for all concentrations for } \\
\text { suspension of rigid spheres; }\end{array}$ & $\begin{array}{l}\text { Thomas } \\
(1965)\end{array}$ \\
\hline E8 & $\mu_{b}=\mu_{f}\left(1+0.75 \frac{\varepsilon_{p} / \varepsilon_{m}}{1-\varepsilon_{p} / \varepsilon_{m}}\right)^{2}$ & $\begin{array}{l}\text { valid for all concentrations for } \\
\text { suspension of bi-disperse spheres; }\end{array}$ & $\begin{array}{l}\text { Chong et al. } \\
\text { (1971) }\end{array}$ \\
\hline E9 & $\begin{array}{l}\mu_{b}=\mu_{f}\left(1-\frac{\varepsilon_{p}}{\varepsilon_{m}}\right)^{-2} \\
\varepsilon_{m}=0.680 \text { for smooth sphere }\end{array}$ & $\begin{array}{l}\text { valid for all concentrations for mono- } \\
\text { disperse suspension of rigid spheres; }\end{array}$ & $\begin{array}{l}\text { Kitano et al. } \\
\qquad(1981)\end{array}$ \\
\hline E10 & $\begin{array}{l}\mu_{b}=\mu_{f}\left\{1+C \frac{3 \pi}{8} \frac{\beta}{\beta+1}\left[\frac{3+4.5 \beta+\beta^{2}}{\beta+1}-\frac{3 \beta+3}{\beta} \ln (\beta+1)\right]\right\} \\
\beta=\frac{\left(\varepsilon_{p} / \varepsilon_{m}\right)^{1 / 3}}{1-\left(\varepsilon_{p} / \varepsilon_{m}\right)^{1 / 3}}\end{array}$ & $\begin{array}{l}\text { only valid for } \varepsilon_{p}>20 \% \text { for mono- } \\
\text { disperse suspension of rigid spheres } \\
\text { with the assumption that the } \\
\text { hydrodynamic interactions between } \\
\text { nearest neighboring particles dominate } \\
\text { the suspension flow; } C \text { is a constant; }\end{array}$ & $\begin{array}{l}\text { Sengun } \\
\text { and } \\
\text { Probstein } \\
(1989)\end{array}$ \\
\hline E11 & $\begin{array}{l}\mu_{b}=\mu_{f}\left(1+\frac{R \varepsilon_{p}}{1-\varepsilon_{p} / \varepsilon_{m}}\right)^{3.3 \varepsilon_{m}} \\
0.7<R<1.25\end{array}$ & $\begin{array}{l}\text { valid for all concentrations for } \\
\text { suspension of bi-disperse spheres; } \\
R \text { is a constant; }\end{array}$ & $\begin{array}{l}\text { Storms et al. } \\
\quad(1990)\end{array}$ \\
\hline E12 & $\mu_{b}=\mu_{f}\left(\begin{array}{l}1.0204+2.8997 \varepsilon_{p} \\
-1.4789 \varepsilon_{p}^{2}+70.183 \varepsilon_{p}^{3}\end{array}\right)$ & $\begin{array}{l}\varepsilon_{p}<40 \% ; \\
\text { valid for suspension of rigid spheres; } \\
\text { the correlation is generated from } \\
\text { experimental data; }\end{array}$ & $\begin{array}{l}\text { Ilic and Phan-Thien } \\
\text { (1994) }\end{array}$ \\
\hline E13 & $\mu_{b}=\mu_{f} e^{5.56 \varepsilon_{m}\left[\left(1-\varepsilon_{p} / \varepsilon_{m}\right)^{-0.45}-1\right]}$ & $\begin{array}{l}\text { valid for all concentrations for } \\
\text { suspension of rigid spheres; } \\
\varepsilon_{m}=0.595 \text { for mono-disperse } \\
\text { suspension; } \\
\varepsilon_{m} \text { is determined by simulation for bi- } \\
\text { disperse suspension; }\end{array}$ & $\begin{array}{l}\text { He et al. } \\
(2001)\end{array}$ \\
\hline
\end{tabular}


formula for both mono- and bi-disperse suspensions and showed good agreement with (Kitano et al., 1981) and (Sengun and Probstein, 1989).

\section{Comparison of different correlations for suspension flow}

To better illustrate the dependence of suspension viscosity on particle concentration, a relative viscosity $\mu_{r}$ is defined:

$$
\mu_{r}=\mu_{b} / \mu_{f}
$$

Figure 2.20 shows all the $\mu_{r}-\varepsilon_{p}$ correlations for suspension of uniform rigid spheres by using a logarithmical scale for $\mu_{r}$ and a linear one for $\varepsilon_{p}$. For lower concentration range $\left(\varepsilon_{p}<20 \%\right)$, most of the correlations give similar results except $E 3$. The correlations diverse considerably from each other in higher concentration range. From an average point of view of all the correlations, we agree with Metzner (1985) that E9 is a very simple correlation and can be considered approximately as the average of all the correlations in the entire concentration range.

\subsubsection{Results}

To see the influence of particle viscosity $\mu_{p}$ on the heat transfer enhancement effect of PCM suspension flow, and to find the best bulk viscosity correlation for our study, three arbitrarily selected values of $\mu_{p}=0.01,0.0046$ and $0.001 \mathrm{~Pa} \cdot \mathrm{s}$ are used in the numerical simulation. To compare with the experimental results provided in section 2.4, the simulations are conducted at the same experimental condition of $L=0.3 \mathrm{~m}$, $D=0.00314 \mathrm{~m}, \varepsilon_{p}=0.15, T_{i n}=298 \mathrm{~K}, d_{p}=2.9 \times 10^{-5} \mathrm{~m}, \mathrm{Re}=22$ and four different wall heat fluxes are selected to find the optimal condition. The results are plotted in 


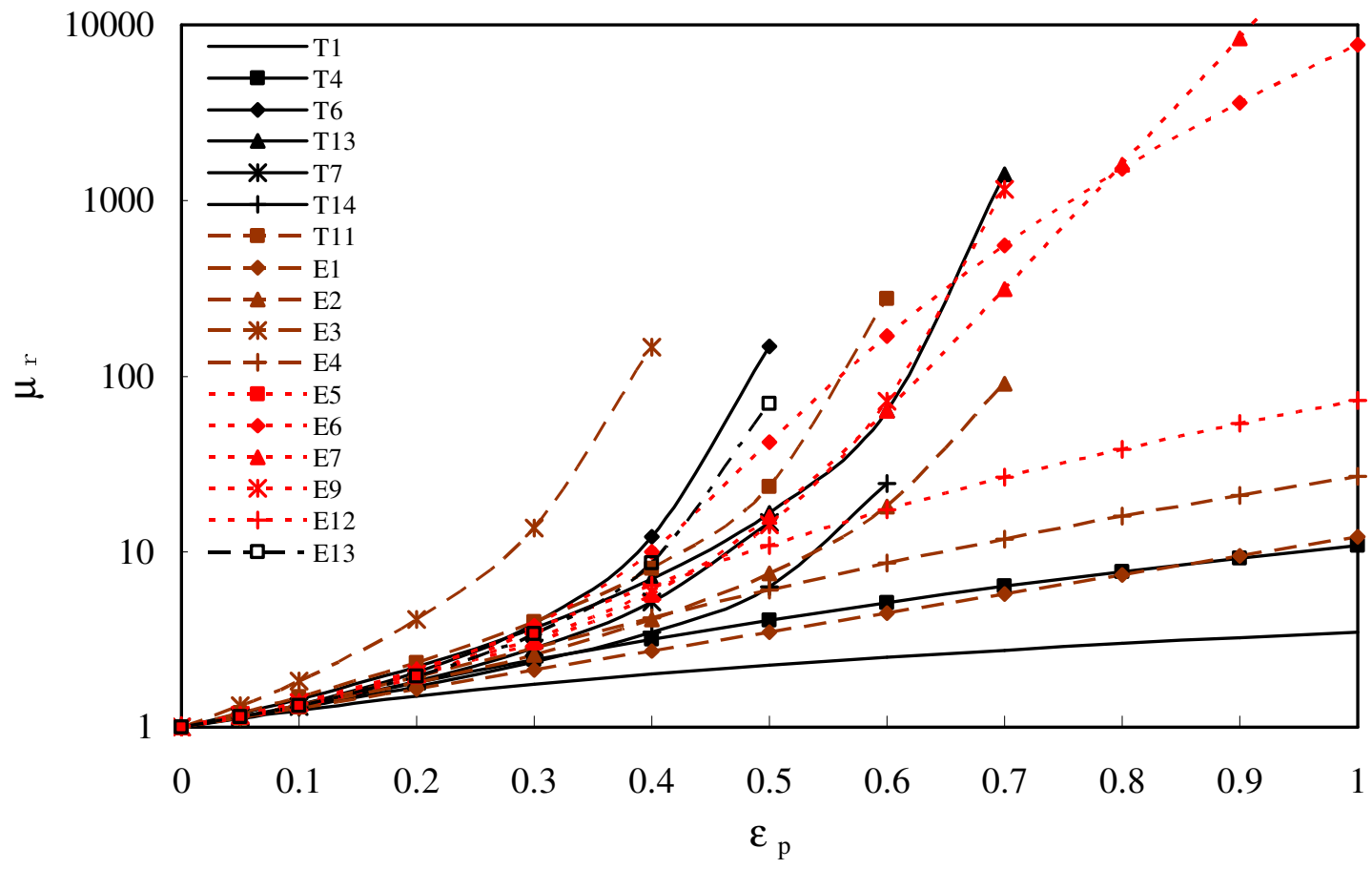

Figure 2.20 Plot of correlations of bulk viscosity for suspension flow.

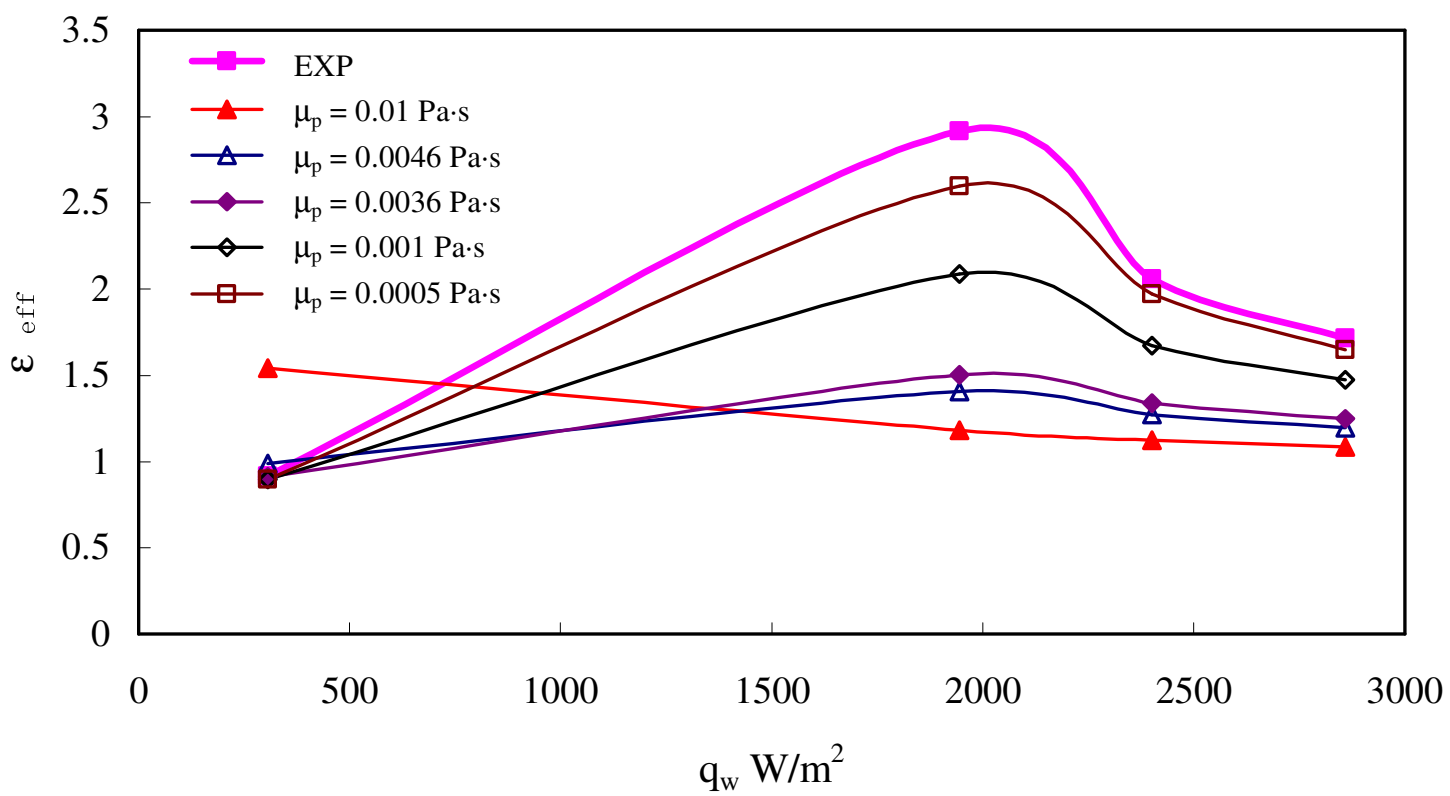

Figure 2.21 Influence of particle viscosity to the heat transfer enhancement effect of PCM suspension flow. 
Fig. 2.21. It shows that the particle viscosity $\mu_{p}$ has a considerable influence on the heat transfer enhancement effect of PCM suspension flow. The previous used the value of $\mu_{p}=0.01 \mathrm{~Pa} \cdot \mathrm{s}$ is clearly not a reasonable value under this experimental condition.

From the previous discussion of different bulk viscosity correlations, we found $E 9$ might be considered the average of all the correlations in the entire concentration range:

$$
\mu_{b}=\mu_{f}\left(1-\frac{\varepsilon_{p}}{0.68}\right)^{-2}
$$

If assume a linear relationship between the bulk viscosity and the particle and fluid viscosities as

$$
\mu_{b}=\varepsilon_{p} \mu_{p}+\left(1-\varepsilon_{p}\right) \mu_{f}
$$

By plugging Eq. (2.43) into Eq. (2.42), we derive a formula to calculate the particle viscosity $\mu_{p}$ :

$$
\mu_{p}=\mu_{f}\left[\left(1-\frac{\varepsilon_{p}}{0.68}\right)^{-2}-\left(1-\varepsilon_{p}\right)\right] / \varepsilon_{p}
$$

For $\varepsilon_{p}=0.15$, Eq. (2.44) gives $\mu_{p}=0.0047 \mathrm{~Pa} \cdot \mathrm{s}$, which is very close to one of the arbitrarily selected values of $\mu_{p}=0.0046 \mathrm{~Pa} \cdot \mathrm{s}$. From Fig. 2.21, we can see that for $\mu_{p}=0.0046 \mathrm{~Pa} \cdot \mathrm{s}$, the simulation results have the same trend as the experiment, which proves Eq. (2.44) is a better approximation for particle viscosity than $\mu_{p}=0.01 \mathrm{~Pa} \cdot \mathrm{s}$.

Although $E 9$ gives a better approximation for $\mu_{p}$, it is not the best correlation for our application. By analyzing the trend in Fig. 2.21, we find that the correlation giving lower viscosity values agrees better with the experimental data. Figure 2.20 shows that 
$E 1$ and $T 1$ give the lowest viscosity values. Because $T 1$ is only valid in very low concentration, $E 1$ is the best correlation for our purpose:

$$
\mu_{b}=\mu_{f} e^{2.5 \varepsilon_{p}}
$$

By also assuming a linear relationship between the bulk viscosity and the particle and fluid viscosities, another formula can be derived for $\mu_{p}$ :

$$
\mu_{p}=\mu_{f}\left[e^{2.5 \varepsilon_{p}}-\left(1-\varepsilon_{p}\right)\right] / \varepsilon_{p}
$$

For $\varepsilon_{p}=0.15$, Eq. (2.46) gives $\mu_{p}=0.0036 \mathrm{~Pa} \cdot \mathrm{s}$. From Fig. 2.21, we find $\mu_{p}=0.0036 \mathrm{~Pa} \cdot \mathrm{s}$ approaches the experimental data best of all the correlations.

To get a better approximation to the experimental data, another particle viscosity value of $\mu_{p}=0.0005 \mathrm{~Pa} \cdot \mathrm{s}$ is also used in the simulation. From Fig. 2.21, this value can give a much better approximation to the experimental data compared to other particle viscosity values. This particle viscosity value is even lower than the water viscosity of $\mu_{f}=0.001 \mathrm{~Pa} \cdot \mathrm{s}$. In our numerical model, there is no numerical limitation on the value range of $\mu_{p}$. From the physical sense, whether or not the particle viscosity can be lower than the water viscosity is still an open question. Therefore, to some extent, we can conclude that to better approximate the experimental data in the current condition, the particle viscosity value should be lower than the water viscosity value in our model.

Equation (2.46) is derived by assuming a linear relationship between the bulk viscosity and the particle and fluid viscosities. With a different relationship assumption, the results might be different and it's possible that there exist a relationship that agrees 
with the experimental data better. This might explains the difference between simulation results and experimental data in Fig. 2.21.

\subsubsection{Conclusions}

In this investigation, a thorough review is given to the available correlations for bulk viscosity of rigid spheres suspensions. By using arbitrarily selected values of $\mu_{p}$ in the simulation, it was found that $\mu_{p}$ has a considerable influence on the heat transfer enhancement effect of PCM suspension flow. The previously used value of $\mu_{p}=0.01 \mathrm{~Pa} \cdot \mathrm{s}$ is not a reasonable value under our experimental condition. A new correlation for $\mu_{p}$ is suggested through comparison with the experiment. It is also found that a particle viscosity value which is lower than water viscosity, will give a better approximation to the experimental data under the given conditions.

\subsection{Conclusions}

In this chapter, a detailed parametric study was conducted for the PCM suspension flow in a micro-channel by using Hao and Tao's two-phase non-thermal-equilibrium based continuum model. By using octadecane as the PCM particle in a micro-channel, the optimum relation between the channel design parameters $L$ and $D$, particle volume fraction $\varepsilon_{p}$, Reynolds number Re, and the wall heat flux $q_{w}$ was found under normal conditions of $\operatorname{Re}=90-600$ and $\varepsilon_{p} \leq 0.25$, as well as extreme conditions such as low Reynolds number $\operatorname{Re}<50$ and high PCM concentration $\left(\varepsilon_{p}=50 \%-70 \%\right)$ slurry flow. The preliminary experimental results proved the existence of the optimal conditions as predicted by the numerical simulation. A correlation between the maximum effectiveness 
factor, PCM particle concentration, and Reynolds number was generated. This correlation can be reasonably used by designer in designing a PCM fluid micro-channel heat sink. The influence of the particle viscosity $\mu_{p}$ to the heat transfer effect of the PCM slurry was discussed based a review to the available suspension bulk viscosity correlations. The results could help the designer in design a high performance phase change micro-channel heat sink. 


\section{CHAPTER 3}

\section{THE LATTICE BOLTZMANN METHOD}

\subsection{INTRODUCTION}

Ever since its birth about two decades ago (1988), The lattice Boltzmann method has successfully found its applications in fluid flow and heat transfer related simulations, which are traditionally covered by computational fluid mechanics (CFD). It is well known that the conventional CFD numerical scheme is based on the discretizations of macroscopic continuum Navier-Stokes (N-S) equations. However, the LBM is based on mesoscopic kinetic equations. In the LBM, the continuum mechanism of the system is simulated by using the collective behavior of all the particles in the system; thus, it can reveal the mechanisms of the research problems from the mesoscopic level (Chen and Doolen, 1998; Yuan, 2005; Inamuro, et al., 2004).

The applications of the LBM are very diverse; it has been applied to a variety of flow fields, such as turbulence and large eddy simulation, flow in dynamics geometry (blood flow), underground water circulation, etc. The LBM has been particularly successful in the area of complex fluids including multiphase/multicomponent fluids, particle suspension flow, and magnetic fluids. This chapter will give an introduction to the methodology and general concepts of the LBM, together with the introduction to different lattice Boltzmann models and their applications in some benchmark cases.

\subsection{FROM LATTICE GAS AUTOMATA TO LATTICE BOLTZMANN METHOD}

In retrospect, the LBM was born from lattice gas automata (LGA). LGA can be considered as discrete particle kinetics on a discrete lattice and time. The LGA method 
was first introduced by Hardy et al. (1976) for studying transport properties of fluids. In 1986, Frisch, Hasslacher and Pomeau (1986) (known as the FHP model) proposed the first two-dimensional LGA model. The FHP model was the first model in history which can correctly recover the Navier-Stokes equations on a hexagonal lattice. Also in 1986, d'Humières et al. (1986) proposed the first three-dimensional LGA model by using the four-dimensional face-centered-hyper-cubic (FCHC) lattice (Chen and Doolen, 1998; Yuan, 2005).

The LGA method is often called the lattice gas cellular automata (LGCA) because it is constructed as a simplified, fictitious molecular dynamic with discrete space, time, and particle velocities. In LGA, each lattice node is occupied by particles with different velocities. A set of Boolean variables $n_{i}(\mathbf{x}, t)(i=1, \ldots, N)$ (also called the particle occupation variables) is defined to describe the particle occupation at each lattice node. Here, $\mathbf{x}$ denotes the particle position, $t$ is the discrete time, $N$ is the number of particle velocities, and $i$ is an index for particle velocity in different directions. The evolution equation of LGA is as follows:

$$
n_{i}\left(\mathbf{x}+\mathbf{e}_{i}, t+1\right)=n_{i}(\mathbf{x}, t)+\Omega_{i}(n(\mathbf{x}, t))
$$

where $\Omega_{i}$ is the collision operator and $\mathbf{e}_{i}$ are the local particle velocities. Starting from an initial state, the configuration of particles at each time step evolves in two sequential substeps: (a) streaming, in which each particle moves to the nearest node in the direction of its velocity, and (b) collision, which occurs when particles arriving at a node interact and change their velocity directions according to collision rules. The collision rule is crucial in LGA because it has to guarantee the conservation of mass, momentum and energy. 
Furthermore, for simplicity, the exclusion principle (no more than one particle being allowed at a given time and node with a given velocity) is imposed. That means the value of $n_{i}$ is either 0 or 1 in the $i$ th direction.

The main advantages of the LGA to fluid dynamics are exact computing (round-off freedom) and virtually unlimited parallelism. But it also suffers from some drawbacks such as large statistical noise and lack of Galilean invariance. These shortcomings have greatly hampered its development as a good model in practical applications. To overcome the above shortcomings, the lattice Boltzmann model was developed.

The basic idea of the LBM is to replace the particle occupation variables $n_{i}$ (Boolean variables) in the evolution equation by single particle distribution functions (real variables) $f_{i}=\left\langle n_{i}\right\rangle$ and neglect individual particle motion and particle-particle correlations in the kinetic equations (McNamara and Zanetti, 1988), where \langle\rangle denotes an ensemble average.

$$
f_{i}\left(\mathbf{x}+\mathbf{e}_{i} \delta t, t+\delta t\right)=f_{i}(\mathbf{x}, t)+\Omega_{i}(f(\mathbf{x}, t))
$$

where $f_{i}$ is the particle distribution function along the $i$ th-direction, and $\Omega_{i}$ is the collision operator. This procedure greatly eliminates statistical noise in the LBM.

Higuera and Jiménez (1989) linearized the collision operator by assuming that the distribution is close to the local equilibrium state. Higuera et al. (1989) proposed an enhanced collision operator approach which is linearly stable. Qian et al. (1992) and Chen et al. (1992) further simplified the collision operator by using the single relaxation time approximation known as the Bhatnagar, Gross and Krook (BGK) approximation. The use of the BGK collision model eliminates the Galilean invariance and velocity- 
dependence of pressure in the LGA model. Moreover, it also allows calculating numerical viscosities from the relaxation parameter, thus making simulation of flow with Reynolds number possible. The lattice Boltzmann model with the BGK approximation is called the lattice BGK (LBGK) model and it is the most widely used model in lattice Boltzmann simulations (Chen and Doolen, 1998; Yuan, 2005).

\subsection{FROM THE CONTINUUM BOLTZMANN EQUATION TO LATTICE BOLTZMANN EQUATION}

From the previous section, we know that the development of the LBM is independent of the continuum Boltzmann equation, because it originated from the LGA. However, later on, it has been proved that the LBM can also be derived from the continuum Boltzmann equation with the BGK collision model (Sterling and Chen, 1996; He and Luo, 1997; Huang, 2007).

The classical Boltzmann equation is an integral-differential equation for the single particle distribution function $f(\mathbf{x}, \mathbf{v}, t)$. By neglecting the force term, the Boltzmann equation can be written as

$$
\frac{\partial f}{\partial t}+\mathbf{v} \nabla f=Q(f, f)
$$

where $\mathbf{x}$ is the particle position in physical space, $\mathbf{v}$ is the particle velocity and $Q$ is the collision integral. By using BGK approximation for the collision term, the BoltzmannBGK equation can be written as:

$$
\frac{\partial f}{\partial t}+\mathbf{v} \nabla f=-\frac{1}{\lambda}\left[f-f^{e q}\right]
$$


where $f^{e q}$ is the equilibrium distribution function (the Maxwell-Boltzmann distribution function) and $\lambda$ is the relaxation time. To solve for $f$ numerically, Eq. (3.4) is first discretized in the momentum space using a finite set of velocities $\left\{\mathbf{v}_{i}\right\}$ without violating the conservation laws:

$$
\frac{\partial f_{i}}{\partial t}+\mathbf{v}_{i} \nabla f_{i}=-\frac{1}{\lambda}\left[f_{i}-f_{i}^{e q}\right]
$$

where $f_{i}(\mathbf{x}, t) \equiv f_{i}\left(\mathbf{x}, \mathbf{v}_{i}, t\right)$ and $f_{i}^{e q}(\mathbf{x}, t) \equiv f_{i}^{e q}\left(\mathbf{x}, \mathbf{v}_{i}, t\right)$ are the distribution function and the equilibrium distribution function of the $i$ th discrete velocity $\mathbf{v}_{i}$, respectively. Equation (3.5) can be further discretized in physical space $\mathbf{x}$ and time $t$. The completely discretized form of Eq. (3.5), i.e. the lattice Boltzmann equation is

$$
f_{i}\left(\mathbf{x}+\mathbf{e}_{i} \delta t, t+\delta t\right)-f_{i}(\mathbf{x}, t)=-\frac{1}{\tau}\left[f_{i}(\mathbf{x}, t)-f_{i}^{e q}(\mathbf{x}, t)\right] \quad(i=0,1, \ldots, N)
$$

where $N$ is the number of discrete velocity directions and $\tau=\lambda / \delta t$ is the nondimensional relaxation time. Equation (3.6) is the discrete lattice Boltzmann equation with the BGK approximation. It is usually called the LBGK model. Equation (3.6) consists of two parts: the left hand side is the streaming part and the right hand side is the collision part. From Eq. (3.6), it can be seen that the collision step is purely local, and the streaming step is a uniform data shifting and requires little computational effort. Equation (3.6) is explicit, easy to implement and straightforward for parallel computation (Yuan, 2005; Huang, 2007).

\subsection{FORMULATION OF THE LBM}

Because of its simplicity and efficiency, the LBGK model has been widely used. There are different LBGK models, usually denoted as $\mathrm{D} n \mathrm{Q} m$, where $n$ is the space 
D2Q7

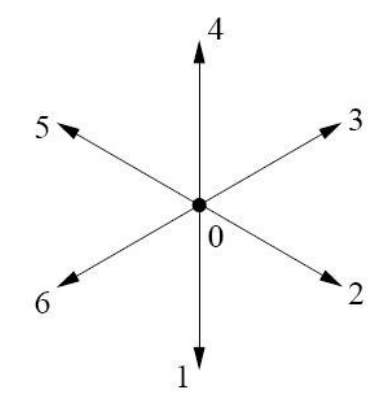

$L_{-v_{x}}^{2}$

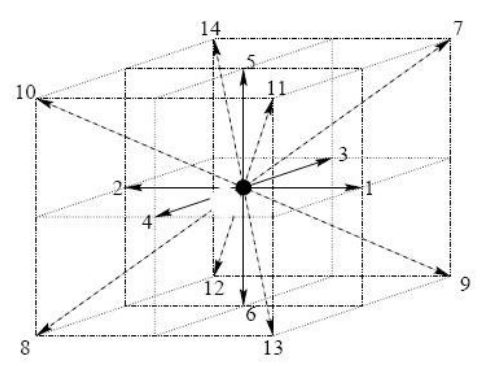

D3Q15
D2Q9
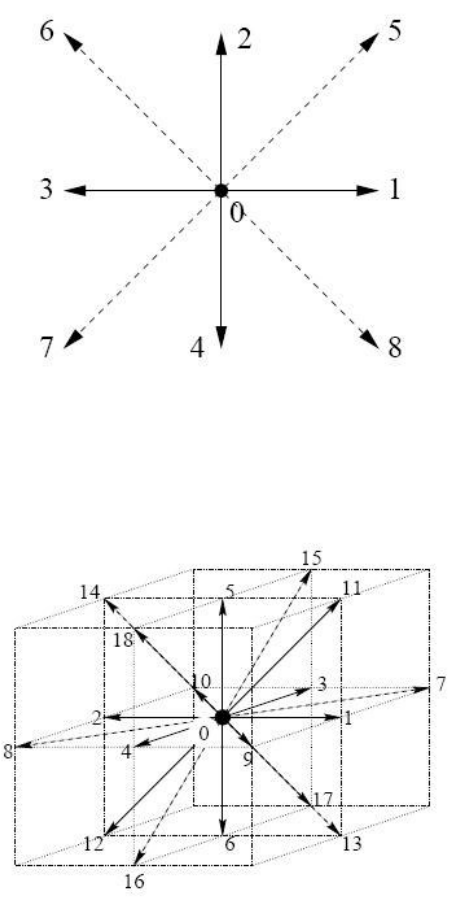

D3Q19

Figure 3.1 Sketches of the most common lattices.

(Source: Dupuis, 2002, p.22)

dimension and $m$ is the number of velocities. For 2D application, the 9 velocity model on a 2D square lattice, called D2Q9 model, has been widely used. For 3D application, there are several cubic lattice models, such as the D3Q15 and the D3Q19 model. Figure 3.1 presents the most commonly used lattices. In our simulations, the D2Q9 model is used throughout. The complete formulation of the $\mathrm{D} 2 \mathrm{Q} 9$ model is as follows:

The lattice Boltzmann equation:

$$
f_{i}\left(\mathbf{x}+\mathbf{e}_{i} \delta t, t+\delta t\right)=f_{i}(\mathbf{x}, t)-\frac{1}{\tau}\left[f_{i}(\mathbf{x}, t)-f_{i}^{e q}(\mathbf{x}, t)\right] \quad(i=0,1, \ldots, 8)
$$

The equilibrium distribution function: 


$$
f_{i}^{e q}=w_{i} \rho\left[1+\frac{3}{c^{2}} \mathbf{e}_{i} \cdot \mathbf{u}+\frac{9}{2 c^{4}}\left(\mathbf{e}_{i} \cdot \mathbf{u}\right)^{2}-\frac{3}{2 c^{2}} \mathbf{u}^{2}\right]
$$

where $w_{i}$ is the weighting factor, $c=\delta x / \delta t$ is the lattice speed, $\delta x$ and $\delta t$ are the lattice spacing and time step, respectively, $\rho$ is the density and $\mathbf{u}$ is the macroscopic velocity.

The discrete velocity set:

$$
\mathbf{e}_{i}= \begin{cases}(0,0), & i=0 \\ ( \pm 1,0) c,(0, \pm 1) c, & i=1,2,3,4 \\ ( \pm 1, \pm 1) c, & i=5,6,7,8\end{cases}
$$

The weighting factor is to ensure the mass and momentum conservation:

$$
w_{i}= \begin{cases}4 / 9, & i=0 \\ 1 / 9, & i=1,2,3,4 \\ 1 / 36, & i=5,6,7,8\end{cases}
$$

The density and macroscopic velocity are determined in terms of the particle distributions from the laws of mass and momentum conservation:

$$
\begin{aligned}
& \rho=\sum_{i=0}^{8} f_{i} \\
& \rho \mathbf{u}=\sum_{i=0}^{8} f_{i} \mathbf{e}_{i}
\end{aligned}
$$

The pressure and the kinematic viscosity are given by:

$$
\begin{aligned}
& p=c_{s}^{2} \rho \\
& \nu=c_{s}^{2} \delta t\left(\tau-\frac{1}{2}\right) \\
& c_{s}^{2}=c^{2} / 3
\end{aligned}
$$


where $c_{s}$ is the lattice speed of sound. To make sure the viscosity is positive, it is obvious that $\tau>1 / 2$.

\subsection{BOUNDARY CONDITIONS}

Boundary condition treatment is very important in any numerical simulation. For the LBM, to some extent, developing accurate and efficient boundary conditions is as important as developing an accurate computation scheme itself, since they will influence the accuracy and stability of the computation (Maier et al., 1996; Ziegler, 1993; Ginzbourg and d'Humières, 1995; Yuan, 2005). In this section, the most commonly used boundary conditions treatment in LBM will be discussed and formulated in detail.

\subsubsection{Bounce-back boundary condition}

Bounce-back boundary condition is the most common and simplest non-slip wall boundary conditions used in the LBM simulation. The fullway bounce-back boundary condition directly comes from LGA. In the fullway bounce-back scheme, when a fluid particle collides with a wall node, it will scatter back to the fluid nodes along its incoming direction, thus, the mean effect yields $u=0$ at the wall (Fig. 3.2(a)). The advantages of the fullway bounce-back boundary condition are its simplicity and conservation of mass and momentum. However, it only gives first order accuracy at the boundaries. In order to achieve second order accuracy, several other wall boundary conditions have also been widely used, especially the extrapolation scheme (Chen et al., 1996) and bounce-back of the non-equilibrium distribution (Zou and He, 1997). The second-order accuracy in space can also be obtained by using the halfway bounce-back scheme. In this scheme, the wall is placed at halfway between a fluid node and a bounceback node (Fig. 3.2(b)). Compared with other second order boundary treatments, the 


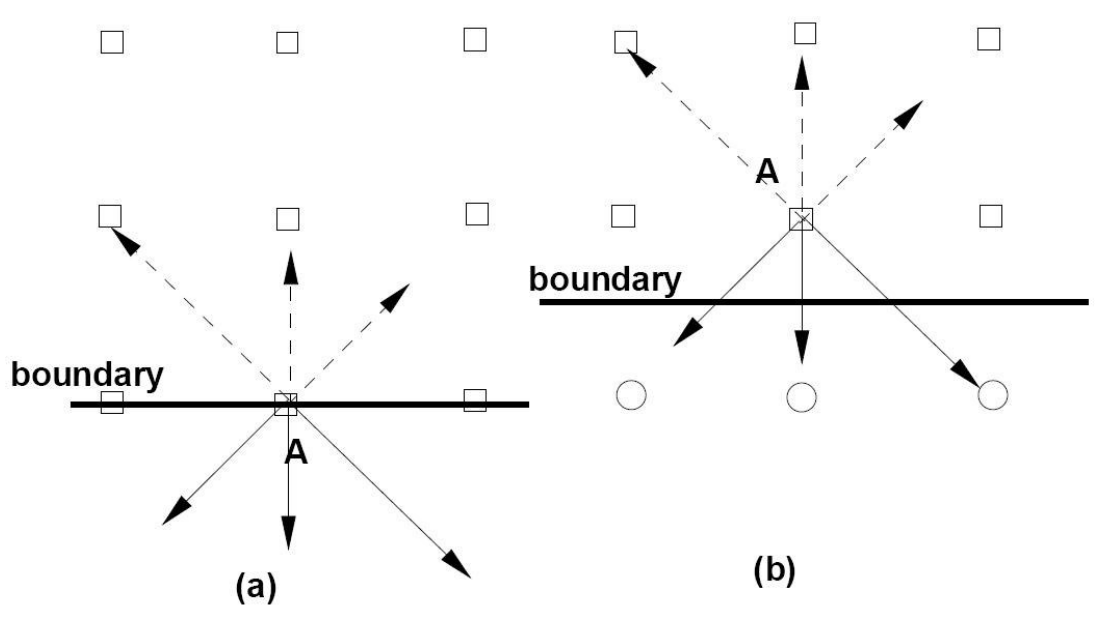

Figure 3.2 Illustration of fullway and halfway bounce-back boundary conditions:

(a) fullway bounce-back;

(b) halfway bounce-back.

(Source: Huang, 2007, p.44)

halfway bounce-back scheme doesn't need any extrapolation and is easy to implement, but it can not deal with a curved wall boundary.

\subsubsection{Periodic boundary condition}

The periodic boundary conditions are the simplest and easiest boundary conditions. The periodic boundary conditions are applied directly to the particle populations, and not to macroscopic flow variables. It can be used as inflow/outflow boundary conditions in the streamwise direction. The uniform body force, such as gravity and pressure gradient, can be easily introduced under this kind of boundary conditions.

\subsubsection{Velocity boundary condition}

Zou and He (1997) proposed a boundary treatment method based on the idea of bounce-back of the non-equilibrium distribution, which can be used to specify both the velocity and pressure boundary conditions in the streamwise direction. 

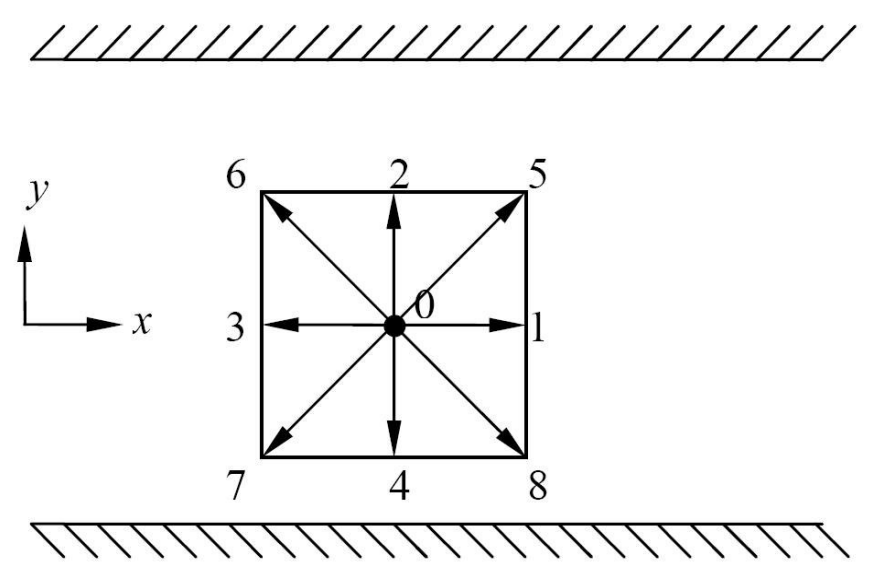

Figure 3.3 Schematic plot of distribution function of D2Q9 model in a channel flow.

To demonstrate how to implement the velocity boundary conditions, we take an inlet node of D2Q9 model (as in Fig. 3.3) as an example:

For the inlet node, after streaming, $f_{0}, f_{2}, f_{3}, f_{4}, f_{6}, f_{7}$ are known and $f_{1}, f_{5}, f_{8}$ are unknown. Suppose $u_{x}, u_{y}$ are specified in the inlet, we can use Eq. (3.11) and Eq. (3.12) to determine $f_{1}, f_{5}, f_{8}$ and $\rho$, which can be put into the form:

$$
\begin{aligned}
& f_{1}+f_{5}+f_{8}=\rho-\left(f_{0}+f_{2}+f_{3}+f_{4}+f_{6}+f_{7}\right) \\
& f_{1}+f_{5}+f_{8}=\rho u_{x}+\left(f_{3}+f_{6}+f_{7}\right) \\
& f_{5}-f_{8}=\rho u_{y}-\left(f_{2}-f_{4}+f_{6}-f_{7}\right)
\end{aligned}
$$

From Eq. (3.16) and Eq. (3.17),

$$
\rho=\frac{1}{1-u_{x}}\left[f_{0}+f_{2}+f_{4}+2\left(f_{3}+f_{6}+f_{7}\right)\right]
$$

By assuming the bounce-back rule is still valid for the non-equilibrium part of the particle distribution function normal to the inlet (in this case, $\left.f_{1}-f_{1}^{e q}=f_{3}-f_{3}^{e q}\right), f_{1}, f_{5}, f_{8}$ can be determined as: 


$$
\begin{aligned}
& f_{1}=f_{3}+\frac{2}{3} \rho u_{x} \\
& f_{5}=f_{7}-\frac{1}{2}\left(f_{2}-f_{4}\right)+\frac{1}{6} \rho u_{x}+\frac{1}{2} \rho u_{y} \\
& f_{8}=f_{6}+\frac{1}{2}\left(f_{2}-f_{4}\right)+\frac{1}{6} \rho u_{x}-\frac{1}{2} \rho u_{y}
\end{aligned}
$$

By the above approach, we can introduce the velocity boundary conditions to the LBM simulation. The velocity boundary conditions for other directions can also be derived in a similar way.

\subsubsection{Pressure boundary condition}

To use Zou and He's (1997) approach to the pressure boundary conditions, we take an exit node of D2Q9 model (as in Fig. 3.3) as an example. Suppose pressure (density) is to be specified at the exit flow boundary, and $u_{y}$ is also specified as $u_{y}=0$. After

streaming, $f_{0}, f_{1}, f_{2}, f_{4}, f_{5}, f_{8}$ are known, in addition to $\rho=\rho_{\text {out }}$ and $u_{y}=0$. We need to determine $u_{x}$ and $f_{3}, f_{6}, f_{7}$ from Eq. (3.11) and Eq. (3.12) as follows:

$$
\begin{aligned}
& f_{3}+f_{6}+f_{7}=\rho_{\text {out }}-\left(f_{0}+f_{1}+f_{2}+f_{4}+f_{5}+f_{8}\right) \\
& f_{3}+f_{6}+f_{7}=-\rho_{\text {out }} u_{x}+\left(f_{1}+f_{5}+f_{8}\right) \\
& f_{6}-f_{7}=\rho_{\text {out }} u_{y}-\left(f_{2}-f_{4}+f_{5}-f_{8}\right)
\end{aligned}
$$

From Eq. (3.23) and Eq. (3.24),

$$
u_{x}=-1+\frac{1}{\rho_{\text {out }}}\left[f_{0}+f_{2}+f_{4}+2\left(f_{1}+f_{5}+f_{8}\right)\right]
$$


By using the bounce-back rule for the non-equilibrium part of the particle distribution function normal to the exit, find $f_{1}-f_{1}^{e q}=f_{3}-f_{3}^{e q}$. With $f_{3}$ known, $f_{6}, f_{7}$ are obtained as:

$$
\begin{aligned}
& f_{3}=f_{1}-\frac{2}{3} \rho_{\text {out }} u_{x} \\
& f_{6}=f_{8}-\frac{1}{2}\left(f_{2}-f_{4}\right)-\frac{1}{6} \rho_{\text {out }} u_{x} \\
& f_{7}=f_{5}+\frac{1}{2}\left(f_{2}-f_{4}\right)-\frac{1}{6} \rho_{\text {out }} u_{x}
\end{aligned}
$$

By the above approach, we can introduce the pressure (density) boundary conditions to the LBM simulation. The pressure boundary conditions for other directions can also be derived in a similar way.

\subsection{THERMAL LATTICE BOLTZMANN MODEL}

\subsubsection{Introduction}

Originally, only mass and momentum conservation were considered in the LBM simulation. However, in many applications it is important and sometimes crucial to consider the thermal effects in fluid flows (Shan, 1997). Although the LBM has met with a significant amount of success in the case of isothermal flows, in general, the simulation of thermal fluid systems by LBM has not achieved the same success as that of isothermal flows. The LBM models for thermal fluid flows have been developed by several groups. In general, these thermal lattice Boltzmann models (TLBM) fall into two categories: the multispeed approach and the passive-scalar approach. The multispeed approach is a straightforward extension of the LB thermal models in which only the density distribution function is used. It implements energy conservation by adding additional discrete 
velocities and by including the higher order velocity terms in the equilibrium distribution. Although theoretically possible, the multispeed approach suffers severe numerical instability and the temperature variation is limited to a narrow range. Some improvement to this approach has been done by Chen and Teixeira (2000) to reduce the stability condition, but unfortunately, they also introduced artificial thermal diffusion.

The passive-scalar approach utilized the fact that the macroscopic temperature satisfies the same evolution equation as a passive scalar if the viscous heat dissipation and compression work done by the pressure are negligible. In a passive-scalar-based TLBM model, the temperature is simulated by using a separate distribution function which is independent of the density distribution function (so it is also called the multidistribution function approach). Thus, the overall complexity of the scheme does not significantly increase. Additionally, unlike the multispeed approach, the thermal diffusivity is independent of the viscosity in the passive-scalar approach, which results in a changeable Prandtl number in simulations. Most importantly, the passive-scalar approach does not explicitly implement energy conservation, and therefore has the same stability as the isothermal LBM models. We will use the passive-scalar approach in our simulations.

\subsubsection{Formulation of the passive-scalar-based TLBM}

In a thermal fluid system, if the viscous and compressive heating effects are negligible, the temperature field satisfies a much simpler passive-scalar equation:

$$
\frac{\partial T}{\partial t}+\mathbf{u} \cdot \nabla T=\nabla \cdot(\alpha \nabla T)+\Psi
$$


where $\mathbf{u}$ is the fluid velocity, $\alpha$ is the thermal diffusivity and $\Psi$ is the source term. In the LBM algorithm, after solving the fluid dynamics part by using Eq. (3.7) and Eq. (3.8), the temperature field can be solved by again using those equations, except that $\tau$ will be replaced by $\tau_{T}$ (the relaxation time for temperature) as follows:

$$
\begin{aligned}
& g_{i}\left(\mathbf{x}+\mathbf{e}_{i} \delta t, t+\delta t\right)=g_{i}(\mathbf{x}, t)-\frac{1}{\tau_{T}}\left[g_{i}(\mathbf{x}, t)-g_{i}^{e q}(\mathbf{x}, t)\right] \quad(i=0,1, \ldots, 8) \\
& g_{i}^{e q}=w_{i} T\left[1+\frac{3}{c^{2}} \mathbf{e}_{i} \cdot \mathbf{u}+\frac{9}{2 c^{4}}\left(\mathbf{e}_{i} \cdot \mathbf{u}\right)^{2}-\frac{3}{2 c^{2}} \mathbf{u}^{2}\right]
\end{aligned}
$$

The temperature is given by:

$$
T=\sum_{i=0}^{8} g_{i}
$$

The thermal diffusivity and Prandtl number are then:

$$
\begin{gathered}
\alpha=c_{s}^{2} \delta t\left(\tau_{T}-\frac{1}{2}\right) \\
\operatorname{Pr}=\frac{v}{\alpha}=\frac{2 \tau-1}{2 \tau_{T}-1}
\end{gathered}
$$

\subsubsection{Boundary conditions}

Three kind of thermal boundary conditions including constant heat flux, constant temperature and adiabatic boundary conditions are used in our simulations. Here we will take the constant heat flux boundary condition as an example to illustrate how to implement them.

For a channel flow like Fig. 3.3, after streaming, the temperature of the inner domain can be obtained. A second order finite difference scheme is used to get the temperature on the wall (Shu et al., 2002). For the bottom wall: 


$$
q=\left.\frac{\partial T}{\partial y}\right|_{i, 1}=\frac{-3 T_{i, 1}+4 T_{i, 2}-T_{i, 3}}{2 \Delta y}
$$

which is then solved for the wall temperature $T_{i, 1}$. For the bottom wall, after streaming, $g_{0}, g_{1}, g_{3}, g_{4}, g_{7}, g_{8}$ are known and $g_{2}, g_{5}, g_{6}$ are unknown. Assume these unknowns can be calculated by their corresponding equilibrium distribution functions given by Eq. (3.32), by summing them together, we have:

$$
g_{2}+g_{5}+g_{6}=\frac{T^{\prime}}{6}\left(1+3 u_{y}+3 u_{y}^{2}\right)
$$

where $u_{y}$ is the velocity normal to the wall. If we know $T^{\prime}$, we will be able to solve for $g_{2}, g_{5}, g_{6}$. Meanwhile, for the bottom wall, from Eq. (3.33), we have:

$$
T_{i, 1}=\sum_{i=0}^{8} g_{i}=g_{0}+g_{1}+g_{2}+g_{3}+g_{4}+g_{5}+g_{6}+g_{7}+g_{8}
$$

By substituting Eq. (3.37) into Eq. (3.38), $T^{\prime}$ can then be solved as follows:

$$
T^{\prime}=\frac{6}{1+3 u_{y}+3 u_{y}^{2}}\left(T_{i, 1}-g_{0}-g_{1}-g_{3}-g_{4}-g_{7}-g_{8}\right)
$$

Finally, $g_{2}, g_{5}, g_{6}$ can be obtained by inserting $T$ into their corresponding form of Eq. (3.32).

The same approach can be easily applied to other thermal boundary conditions. For the constant temperature boundary condition, the wall temperature $T_{i, 1}$ is given; for the adiabatic boundary conditions, just make $q=0$ in Eq. (3.36) to get $T_{i, 1}$; the other part of the derivation is the same for all three conditions. 


\subsection{MULTICOMPONENT LATTICE BOLTZMANN MODEL}

\subsubsection{Introduction}

There are many difficulties in numerically simulating multiphase or multicomponent fluids with conventional methods because not only different phases may merge, separate or break, they may also change from one phase to another. The LBM has shown great potential for the modeling of multiphase and multicomponent fluid flows. Due to its kinetic nature, the LBM is capable of incorporating the interparticle interactions, which are difficult to implement in traditional methods. Therefore, the key step in developing the LBM multiphase and multicomponent models is to correctly incorporate the particle interactions into the evolution of the particle distribution functions, so that the macroscopically correct multiphase and multicomponent flow behavior can be obtained.

There have been a number of LBM multiphase and multicomponent flow models in the literature. The first immiscible multiphase LBM model proposed by Gunstensen et al. (1991) uses red- and blue- colored particles to represent two kinds of fluids. The phase separation is then produced by the repulsive interaction based on the color gradient and color momentum. The model proposed by Shan and Chen $(1993,1994)$ (SC) imposes a non-local interaction between fluid particles at neighboring lattice sites. The interaction potentials control the form of the equation of state of the fluid. Phase separation occurs automatically when the interaction potentials are properly chosen. There is also the socalled free-energy-based approach by Swift et al. (1996). In this model, the description of non-equilibrium dynamics, such as Cahn-Hilliard's approach, is incorporated into the LBM model by using the concepts of the free energy function. However, this model does 
not satisfy Galilean invariance and some unphysical effects will be produced in the simulation. In the multicomponent model proposed by He et al. (1999), two sets of particle distribution functions are employed. The first set is used to simulate pressure and velocity fields and another set is used to capture the interface only. Their approach is more flexible in implementing the thermodynamics of the flow, but with a severe problem of numerical instability.

Among all the LBM multicomponent models, the SC model is widely used due to its simplicity and remarkable versatility: it can handle fluid phases with different densities, viscosities and wettability. Originally, the SC model was proposed for the flow systems with multiple phases and components. Later on, the model was also used in single component multiphase flow systems. In this study, the multicomponent version of the SC model will be employed.

\subsubsection{Formulation of the SC multicomponent LBM model}

This section will give a detailed formulation of the SC model for a two immiscible component system by using D2Q9 LBM model (Shan and Doolen, 1995 and 1996). The lattice Boltzmann equation and its corresponding equilibrium distribution function for a two immiscible component system is as follows:

$$
\begin{aligned}
& f_{i}^{\sigma}\left(\mathbf{x}+\mathbf{e}_{i} \delta t, t+\delta t\right)=f_{i}^{\sigma}(\mathbf{x}, t)-\frac{1}{\tau_{\sigma}}\left[f_{i}^{\sigma}(\mathbf{x}, t)-f_{i}^{\sigma, e q}(\mathbf{x}, t)\right] \quad(i=0,1, \ldots, 8 ; \sigma=1,2) \\
& f_{i}^{\sigma, e q}=w_{i} \rho_{\sigma}\left[1+\frac{3}{c^{2}} \mathbf{e}_{i} \cdot \mathbf{u}_{\sigma}^{e q}+\frac{9}{2 c^{4}}\left(\mathbf{e}_{i} \cdot \mathbf{u}_{\sigma}^{e q}\right)^{2}-\frac{3}{2 c^{2}}\left(\mathbf{u}_{\sigma}^{e q}\right)^{2}\right]
\end{aligned}
$$

where $\sigma=1,2$ denotes the two components, $\tau_{\sigma}$ is the relaxation time for the $\sigma$ th component, $\rho_{\sigma}$ is the mass density of the $\sigma$ th component, the other parameters have the 
same meaning as before. The parameters $\rho_{\sigma}$ and $\mathbf{u}_{\sigma}^{e q}$ in the above equilibrium distribution function are chosen to be:

$$
\begin{aligned}
& \rho_{\sigma}=m_{\sigma} \sum_{i=0}^{8} f_{i} \\
& \rho_{\sigma} \mathbf{u}_{\sigma}^{e q}=\rho_{\sigma} \mathbf{u}^{\prime}+\tau_{\sigma} \mathbf{F}_{\text {total }, \sigma}
\end{aligned}
$$

where $m_{\sigma}$ is the molecular mass of the $\sigma$ th component, $\mathbf{F}_{\text {total }, \sigma}$ is the total force acting on particles of the $\sigma$ th component. $\mathbf{F}_{\text {total }, \sigma}$, includes both external forces and interparticle forces. With $\mathbf{u}_{\sigma}^{e q}$ so chosen, at every site and for each collision step, each component gains an additional momentum $\mathbf{F}_{\text {total }, \sigma}$ due to external and interparticle forces. In the absence of any additional forces, all the components are assumed to have a common averaged velocity $\mathbf{u}^{\prime}$. From the requirement that the total momentum must be conserved at each collision when $\mathbf{F}_{\text {total }, \sigma}=0$, u' can be expressed as

$$
\mathbf{u}^{\prime}=\frac{\sum_{\sigma=1}^{2}\left(\frac{m_{\sigma}}{\tau_{\sigma}} \sum_{i=0}^{8} f_{i}^{\sigma} \cdot \mathbf{e}_{i}\right)}{\sum_{\sigma=1}^{2} \frac{\rho_{\sigma}}{\tau_{\sigma}}}
$$

In general, this averaged velocity is different, and should be carefully distinguished, from the fluid velocity that represents the overall mass transfer. The long range interaction force between particles of component $\sigma$ at site $\mathbf{x}$ and particles of component $\bar{\sigma}$ at site $\mathbf{x}^{\prime}$ is introduced by:

$$
\mathbf{F}_{f, \sigma}(\mathbf{x})=-\psi_{\sigma}(\mathbf{x}) \sum_{\mathbf{x}^{\prime}} \sum_{\bar{\sigma}} G_{\sigma \bar{\sigma}}\left(\mathbf{x}, \mathbf{x}^{\prime}\right) \psi_{\sigma}\left(\mathbf{x}^{\prime}\right)\left(\mathbf{x}^{\prime}-\mathbf{x}\right)
$$


where $G_{\sigma \bar{\sigma}}\left(\mathbf{x}, \mathbf{x}^{\prime}\right)$ is Green's function and satisfies $G_{\sigma \bar{\sigma}}\left(\mathbf{x}, \mathbf{x}^{\prime}\right)=G_{\bar{\sigma} \sigma}\left(\mathbf{x}^{\prime}, \mathbf{x}\right)$. It reflects the intensity of the interparticle interaction, with $G_{\sigma \bar{\sigma}}\left(\mathbf{x}, \mathbf{x}^{\prime}\right)<0$ representing attractive forces between particles. $\psi_{\sigma}(\mathbf{x})$ is called the "effective mass" and is defined as a function of $\mathbf{x}$ through its dependency on the local density, $\psi_{\sigma}(\mathbf{x})=\psi_{\sigma}(\rho(\mathbf{x}))$. In the SC model, the function of $\psi_{\sigma}(\mathbf{x})$ can be varied and different choices will give different equations of state. In the actual simulation, SC introduced the concept of the nearest neighbor interparticle force, which means that only the interactions between the nearest neighbors are considered:

$$
G_{\sigma \bar{\sigma}}\left(\mathbf{x}, \mathbf{x}^{\prime}\right)= \begin{cases}0, & \left|\mathbf{x}-\mathbf{x}^{\prime}\right|>c \\ g_{\sigma \bar{\sigma}}, & \left|\mathbf{x}-\mathbf{x}^{\prime}\right| \leq c\end{cases}
$$

where $c$ is the lattice spacing, and $g_{\sigma \bar{\sigma}}$ represents the strength of interparticle interactions. The constant body forces such as gravity can be expressed as

$$
\mathbf{F}_{b, \sigma}(\mathbf{x})=\rho_{\sigma}(\mathbf{x}) \mathbf{a}_{\sigma}
$$

where $\mathbf{a}_{\sigma}$ is the acceleration due to the body force for the $\sigma$ th component. So the total force acting on particles of component $\sigma$ at site $\mathbf{x}$ is

$$
\mathbf{F}_{\text {total }, \sigma}(\mathbf{x})=\mathbf{F}_{f, \sigma}(\mathbf{x})+\mathbf{F}_{b, \sigma}(\mathbf{x})
$$

The viscosity of the two components can be calculated in the same way as Eq. (3.14):

$$
v_{\sigma}=c_{s}^{2} \delta t\left(\tau_{\sigma}-\frac{1}{2}\right)
$$




\subsection{BENCHMARK TESTS}

By using the model discussed in this chapter, some benchmark tests results are presented below.

\subsubsection{Poiseuille flow test}

The Poiseuille flow in a slit driven by gravity may be the simplest flow system that can be simulated with LBM. It requires only the bounce-back boundaries along the walls and periodic boundaries in the flow direction. We now use the Poiseuille flow to test the basic LBM model given in section 3.5.

Theoretically, the velocity profile in a slit of width $2 a$ is parabolic and given by:

$$
u(x)=\frac{G^{*}}{2 \mu}\left(a^{2}-x^{2}\right)
$$

where $G^{*}$ can be a pressure gradient $\left(G^{*}=\Delta P / L\right)$ or a gravitational gradient $\left(G^{*}=\rho g\right)$.

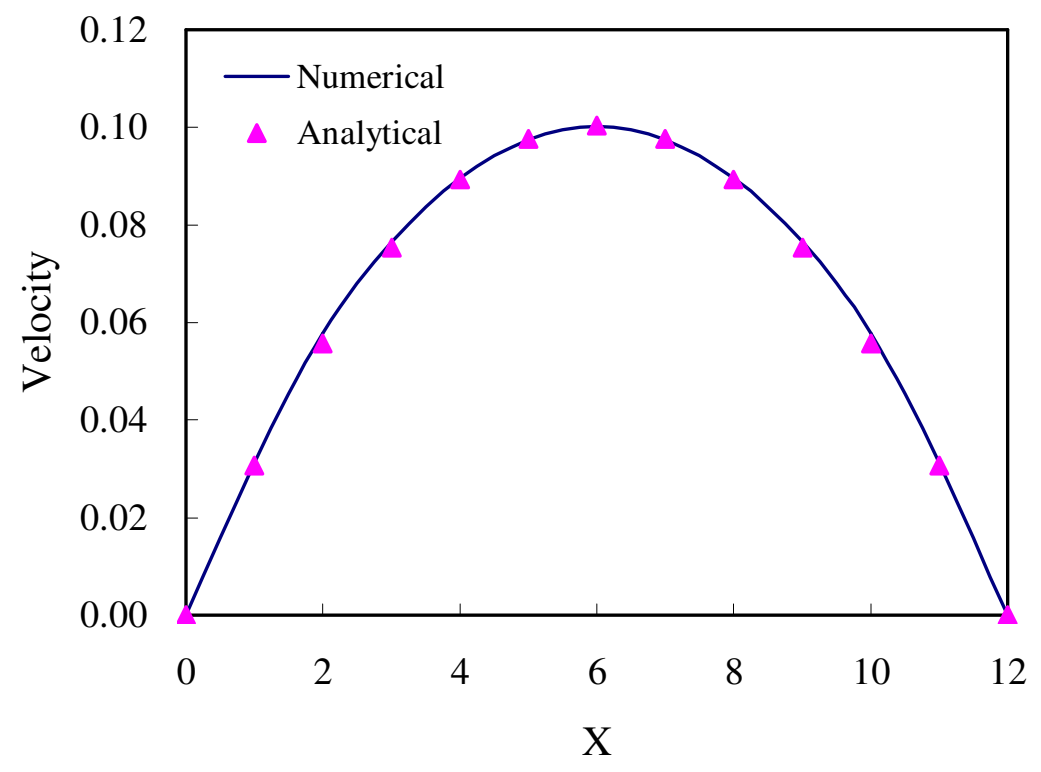

Figure 3.4 Velocity profile for Poiseuille flow from LBM simulation and analytical solution. 
In the simulation, we use the gravity as the driving force for the Poiseuille flow. A lattice size of $12 \times 100$ is used. Fig. 3.4 shows the cross-sectional velocity profile at $\operatorname{Re}=4.4$, and it agrees very well with the analytical solution from Eq. (3.50).

\subsubsection{Rayleigh-Bénard convection test}

To test the TLBM model given in section 3.6, the Rayleigh-Bénard convection is selected as a benchmark test. The Rayleigh-Bénard convection phenomena will happen when a horizontal layer of viscous fluid is heated from the bottom and the top boundary is maintained at a lower temperature. When the temperature difference between the bottom and top boundary exceeds some threshold, the static conduction becomes unstable. Any small perturbation will make the system become convective (Shan, 1997).

In the simulation, the temperature at the bottom wall $(y=0)$ and top wall $(y=1)$ are kept at $T_{B}=1$ and $T_{T}=0$, respectively. So the temperature difference between the walls is $\Delta T=T_{B}-T_{T}=1$. A lattice size of $100 \times 50$ is used in the simulation. The two non-dimensional terms used to describe the system are the Prandtl number and the Rayleigh number. The Prandtl number is defined in Eq. (3.35). The Rayleigh number is defined as

$$
R a=\frac{g \beta \Delta T(n y)^{3}}{v \alpha}
$$

where $g$ is the acceleration due to gravity, $\beta$ is the thermal expansion coefficient, $n y$ is the lattice size in the $y$ direction. The Boussinesq approximation is used, which assumes that the material properties are independent of temperature except in the body force term. Then the external force for this case can be calculated as 


$$
\rho \mathbf{G}=\rho \beta g\left(T-T_{0}\right) \mathbf{j}
$$

where $T_{0}=T_{B}-y \Delta T$. Figure 3.5 plots the typical velocity vectors and isotherms at $R a=5000$ and $\operatorname{Pr}=1$. From Fig. 3.5, we can see that the current TLBM model successfully simulated the typical Rayleigh-Bénard convection phenomena and thus can be used for future research.

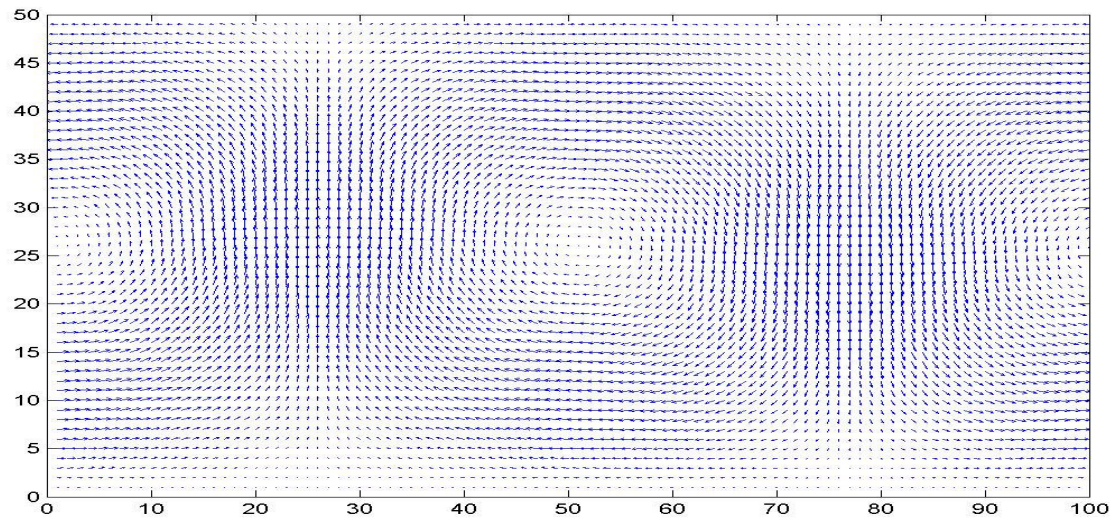

(a)

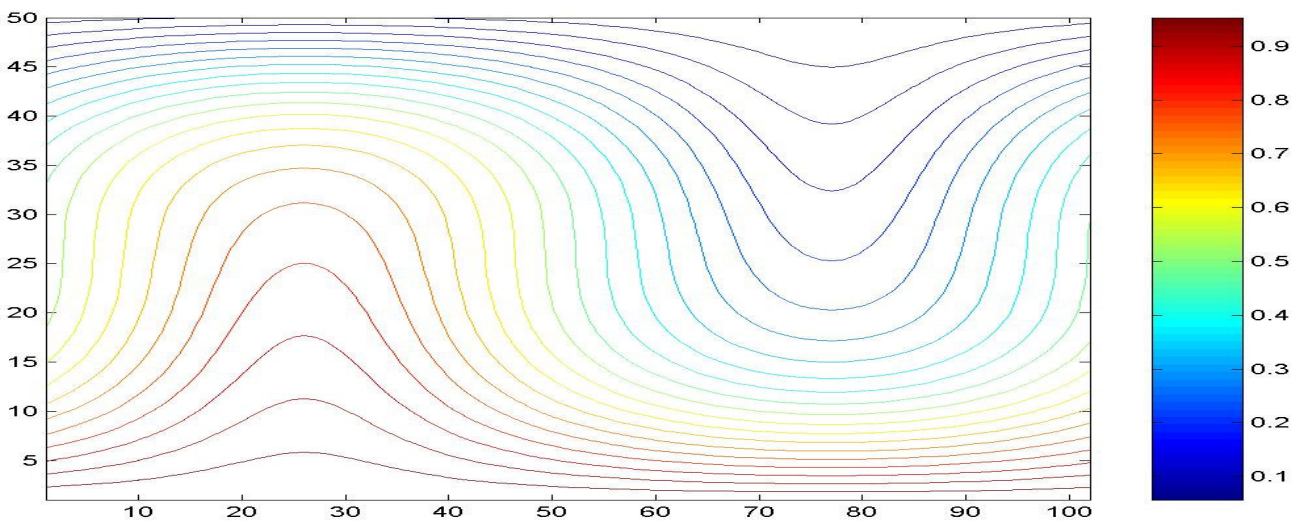

(b)

Figure 3.5 Rayleigh-Bénard convection at $R a=5000$ and $\operatorname{Pr}=1$ :

(a) Velocity vectors; (b) Isotherms. 


\subsubsection{Flow and heat transfer in a symmetric bifurcation channel test}

Bifurcation structures are very common in the human body. Recent research also shows that they have a promising application future in nanotechnology. So it is very important for us to fully understand the flow and heat transfer characteristics inside such a structure. Now we use this symmetric bifurcation simulation as an example to demonstrate the application of the previous LBM and TLBM models.

Consider a model of a two-dimensional symmetric bifurcation that consists of one main tube of diameter $D$ and the length $L$ and two branches at the end of the main tube, each of which has an inner length $L$ and diameter $D / 2$. The bifurcation angle $\theta$ is defined as the angle between each branch and the centerline of the main tube. The geometry of the symmetric bifurcation is illustrated in Fig. 3.6.

The geometry of the symmetric bifurcation makes the region just before the divider an expanding region. As a result, both the pressure and the velocity drop near to the

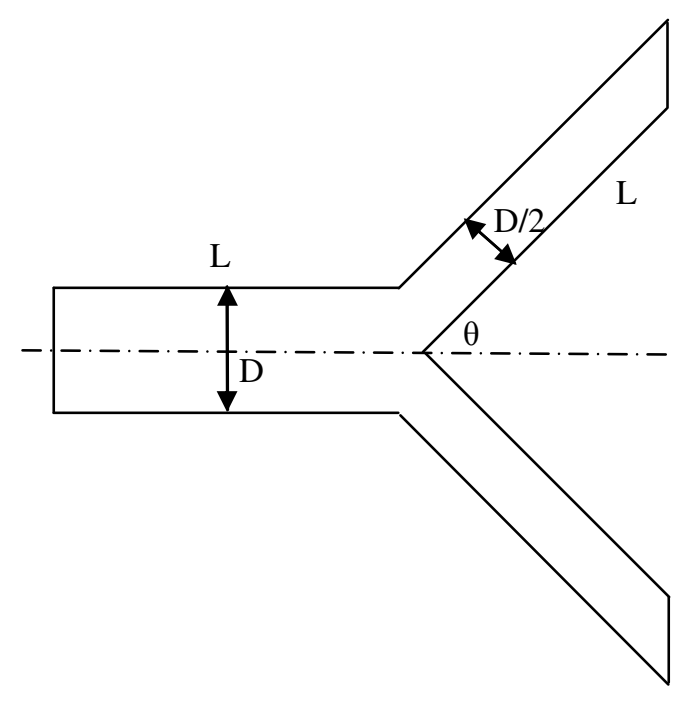

Figure 3.6 The geometry of the simulated symmetric bifurcation. 
divider before they enter the branches, where the velocity accelerates towards the fully developed flow and the pressure drops faster than the pressure in the main branch.

For the flow boundary conditions: at the inlet of the main channel, a uniform velocity of $u_{i n}=0.1$ is set. Therefore, the Reynolds number can be calculated by $R e=D u_{i n} / \nu$. For the outlet, we use a constant pressure boundary condition of $p_{\text {out }}=1 / 3$. For the walls, use simple bounce back rule. The implementation details of these boundary conditions can be seen in section 3.5. For the thermal boundary condition, the inlet is flat temperature boundary condition of $T_{i n}=1.0$, the outlet is set to be adiabatic and all the walls are set to constant heat flux $(q)$ boundary conditions.

In the simulation, the flow and heat transfer characteristics of this symmetric bifurcation structure are thoroughly investigated under different bifurcation angles, Reynolds numbers and wall heat fluxes. Figure 3.7 shows the velocity magnitude, temperature distribution and pressure distribution in the tube at different Reynolds numbers of $\operatorname{Re}=6,12,24$, and under the condition of $\theta=45^{0}$ and $q=1$. From Fig. 3.7(a), we can observe that the flow near the divider becomes complex. As the region before the divider is an expansion region, the velocity flow pattern drops before entering the branches. The velocity skews towards the inner walls inside the bifurcations and each of the two streams are bent because of the influence of the secondary motion, with higher velocities near the outer walls of the bend. All these features are well known and also observed by other researchers (Artoli et al., 2004). Figure 3.7(b) shows that with the increase of Re, the exit temperature will drop, which is correct because with the increase of Re, the fluid will stay a shorter time in the tube and therefore absorb less heat. Figure 
3.7(c) shows the corresponding pressure field for this case. It can be clearly seen that the pressure is recovered around the divider. Figure 3.8 shows the temperature field at different heat flux under the condition of $\theta=45^{\circ}$ and $\operatorname{Re}=6$. When heat flux changes from 1 to 2 to 4 (doubled), the corresponding exit temperature also nearly doubled. Figure 3.9 shows the velocity magnitude, temperature distribution and pressure distribution in the tube at different bifurcation angles of $\theta=30^{\circ}, 45^{\circ}, 60^{\circ}$. The calculation is under the condition of $\operatorname{Re}=6$ and $q=1$. From Fig. 3.9(a), the velocity magnitude fields are almost unchanged with different angles. Figure 3.9(b) shows that exit temperature of $\theta=30^{\circ}$ and $60^{\circ}$ is higher than the $45^{\circ}$ case. That is because the definition of the boundaries for $30^{\circ}$ and $60^{\circ}$ is different from the $45^{\circ}$ case, probably more heat is introduced under these two conditions. Figure 3.9(c) shows the dimensionless inlet average pressure increases as $\theta$ increases. That implies a higher pressure difference is needed to drive the flow for high bifurcation angles.

To further validate our model, a verification case is run by using commercial CFD software FLUENT under the same condition of $\theta=45^{\circ}, \operatorname{Re}=12$ and $q=1$. The comparisons are presented in terms of dimensionless quantities in Fig. 3.10, in which the dimensional heat flux is determined for a desired $42 \mathrm{~K}$ temperature increase in flow. It can be seen that the velocity, temperature, and pressure profiles obtained from LBM are in good agreement with the CFD results (The deviation in the exit mean temperature is within $0.3 \%$ ).

The above simulation demonstrated the applicability of the LBM and TLBM in simulating a single-phase flow under complicated flow and heat boundary conditions. 


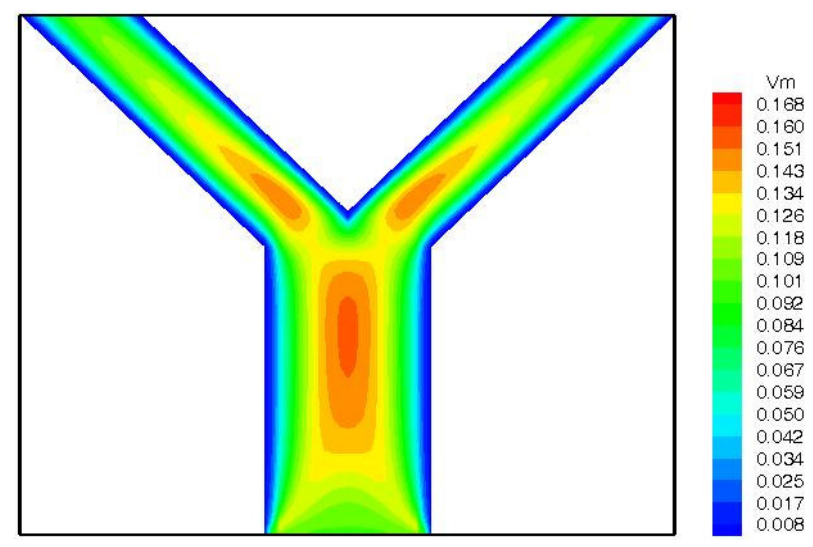

$\mathrm{Re}=6$

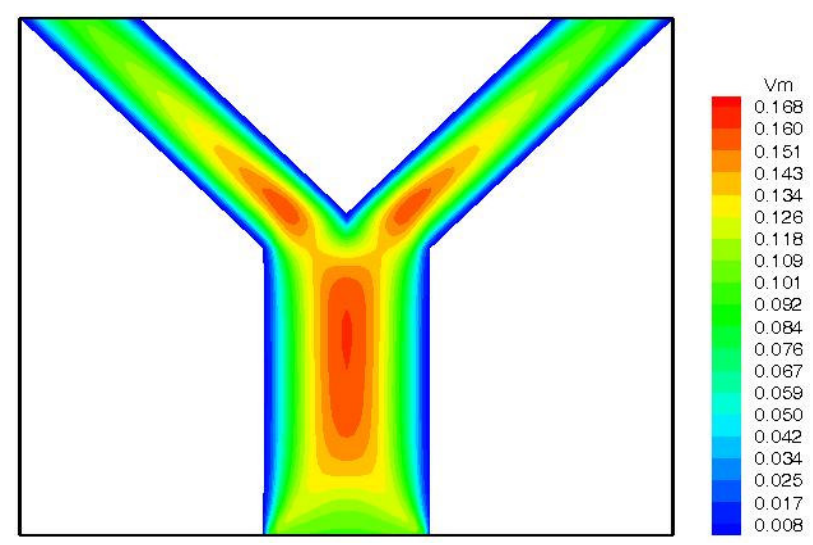

$\mathrm{Re}=12$

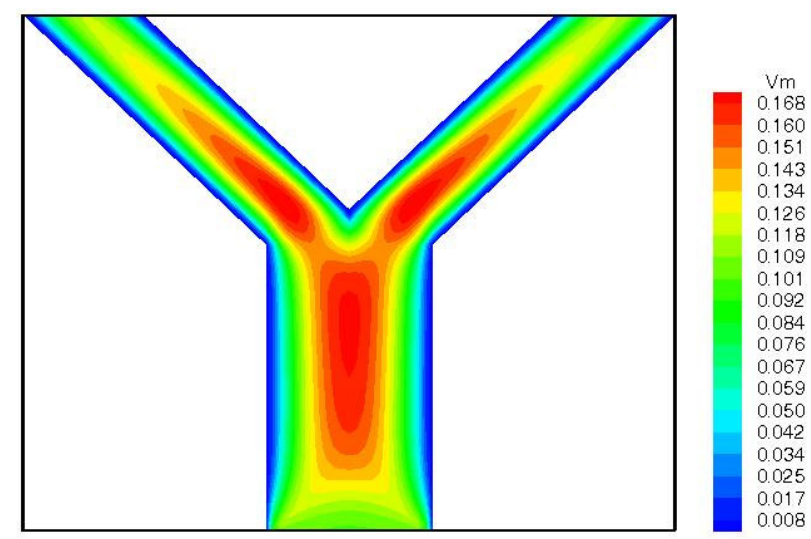

$\operatorname{Re}=24$

Figure 3.7 Simulation results for $\theta=45^{\circ}$ and $q=1$ at different Reynolds numbers: (a) Velocity magnitude. 


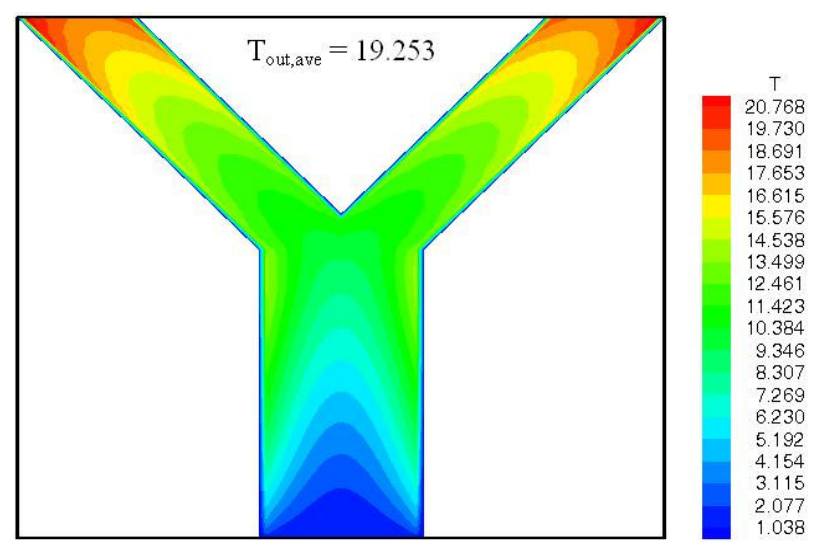

$\operatorname{Re}=6$

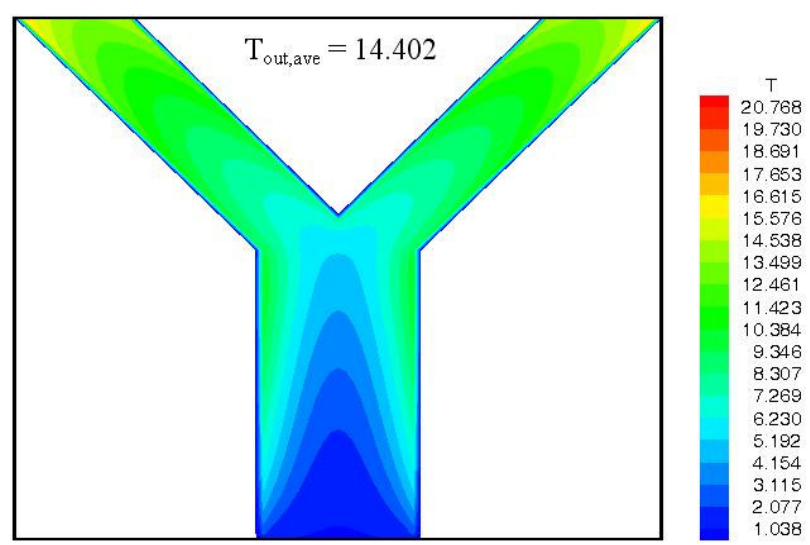

$\operatorname{Re}=12$

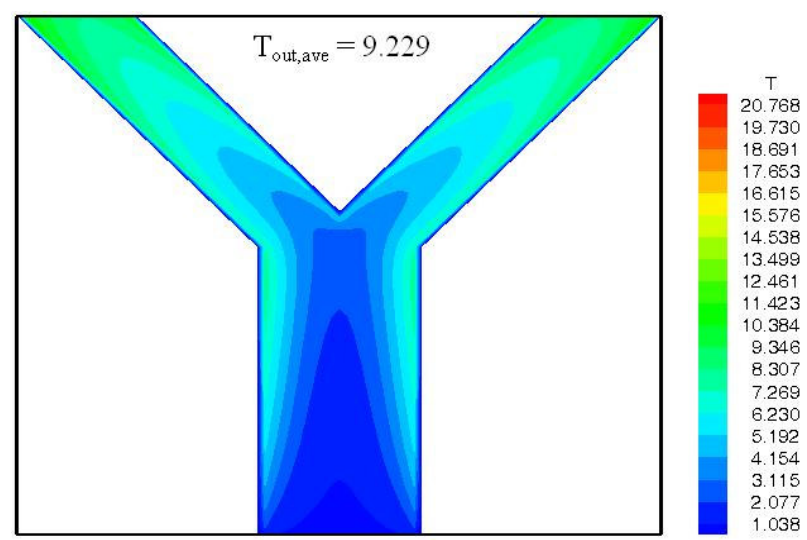

$\operatorname{Re}=24$

Figure 3.7 (Continued) Simulation results for $\theta=45^{\circ}$ and $q=1$ at different Reynolds numbers:

(b) Temperature field. 


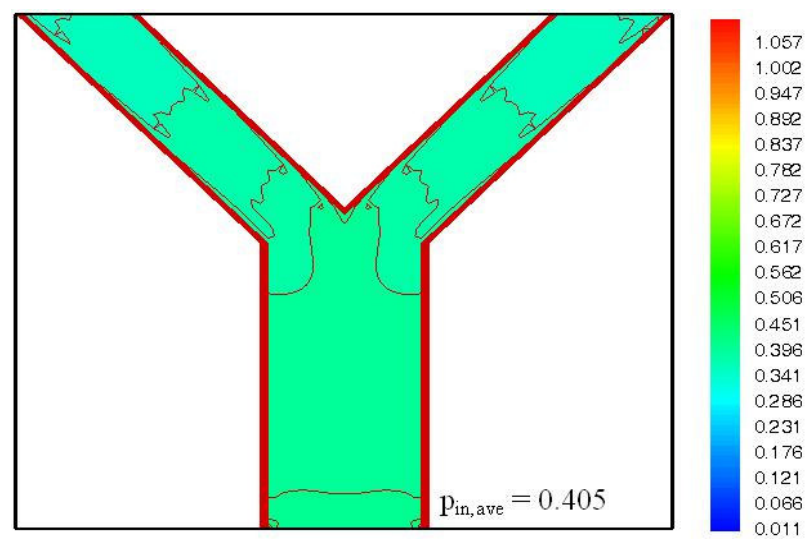

$\operatorname{Re}=6$

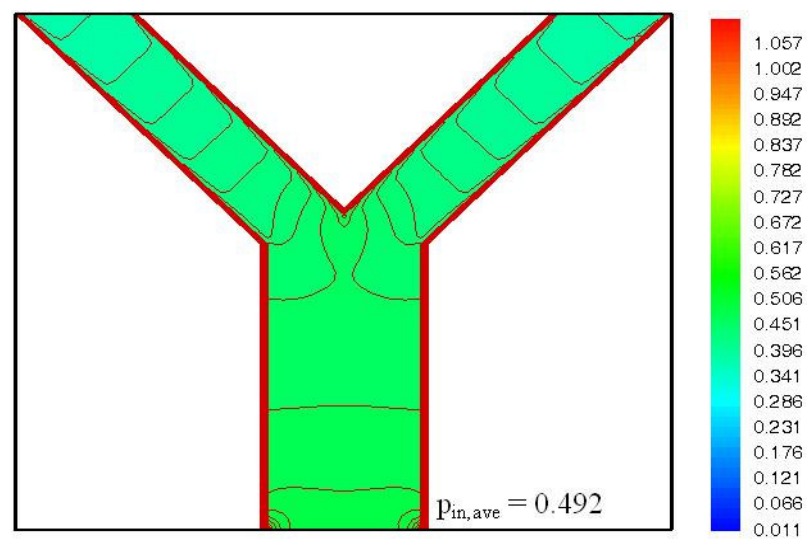

$\mathrm{Re}=12$

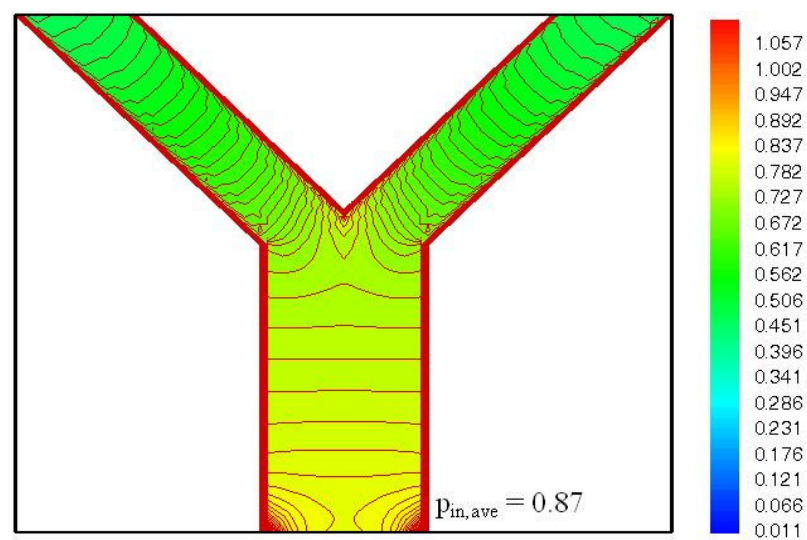

$\operatorname{Re}=24$

Figure 3.7 (Continued) Simulation results for $\theta=45^{\circ}$ and $q=1$ at different Reynolds numbers:

(c) Pressure field. 


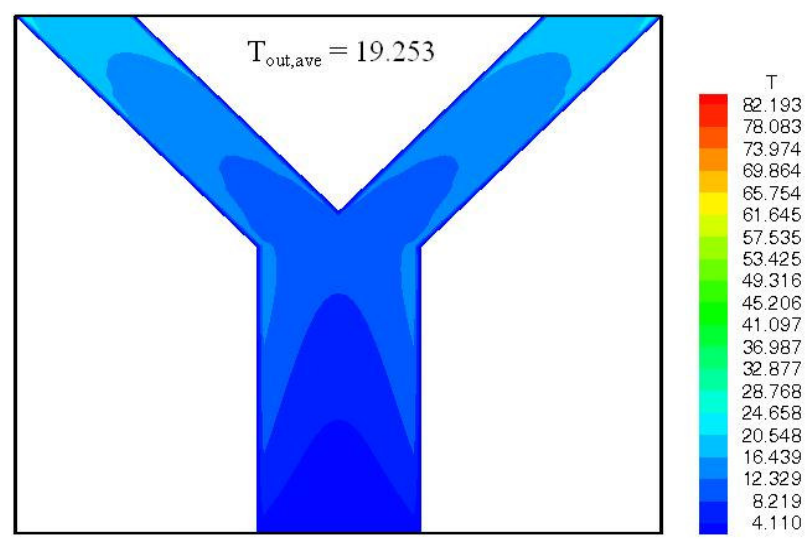

$q=1$

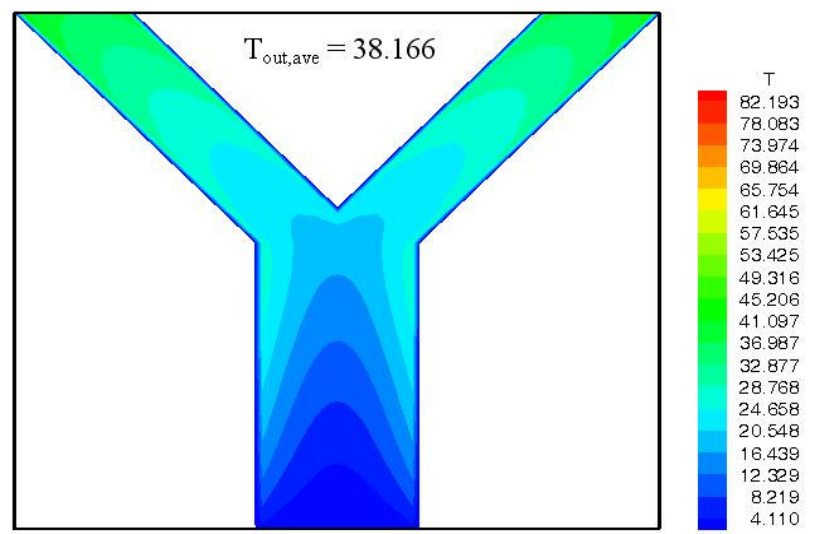

$$
q=2
$$

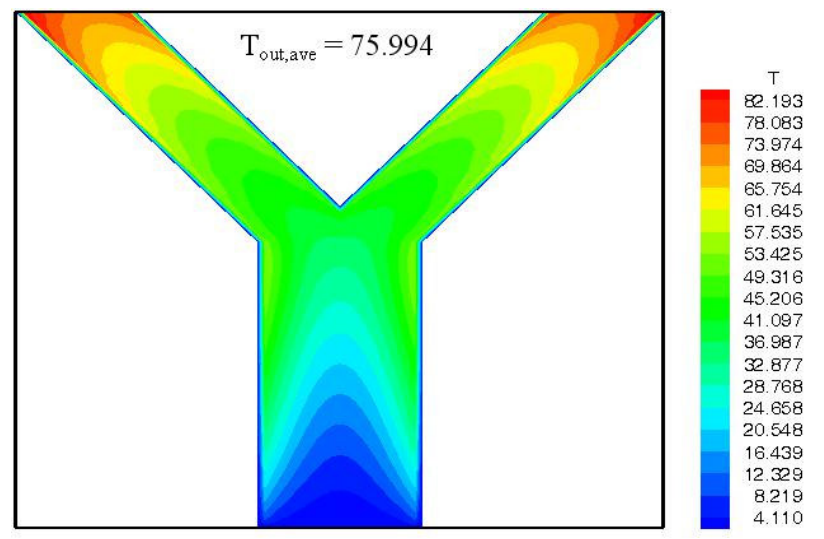

$$
q=4
$$

Figure 3.8 Temperature fields for different heat fluxes at $\theta=45^{\circ}$ and $\operatorname{Re}=6$. 

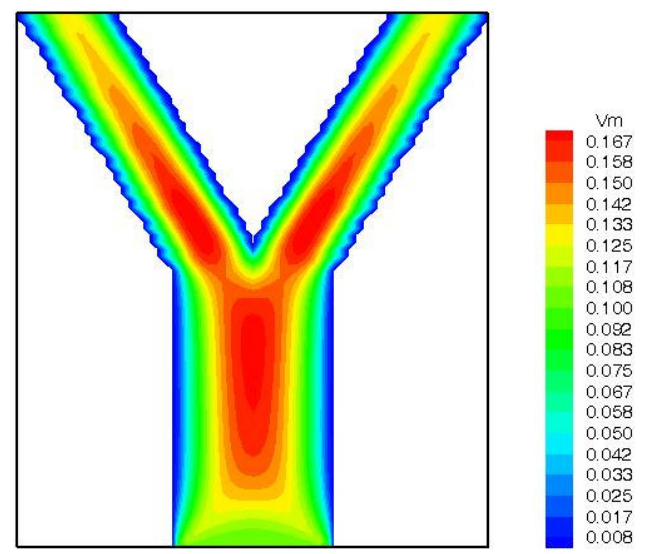

$$
\theta=30^{0}
$$

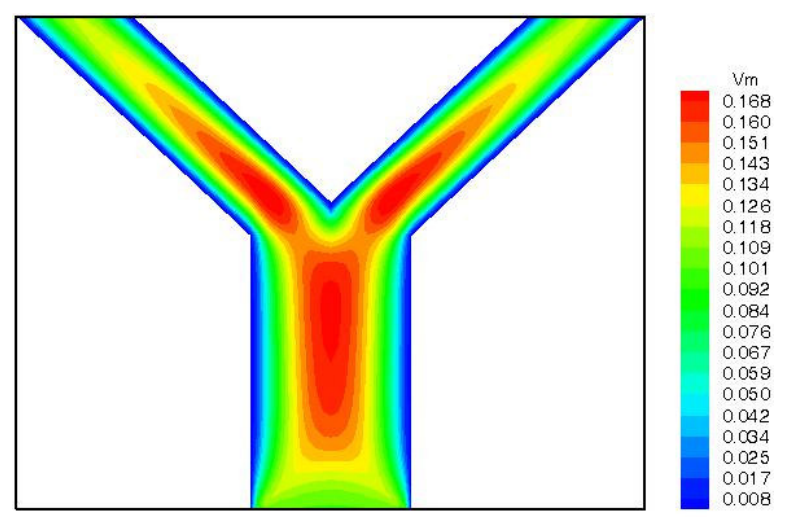

$$
\theta=45^{0}
$$

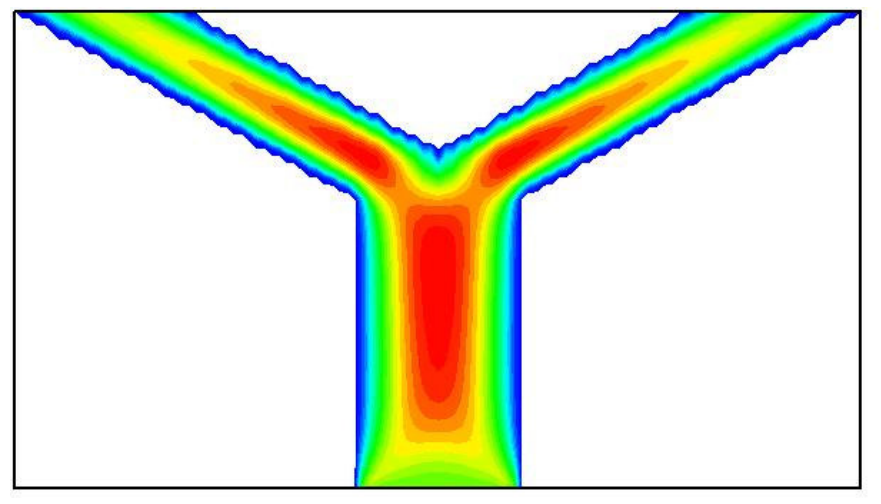

$$
\theta=60^{\circ}
$$

Figure 3.9 Simulation results for $\operatorname{Re}=6$ and $q=1$ at different bifurcation angles:

(a) Velocity magnitude. 


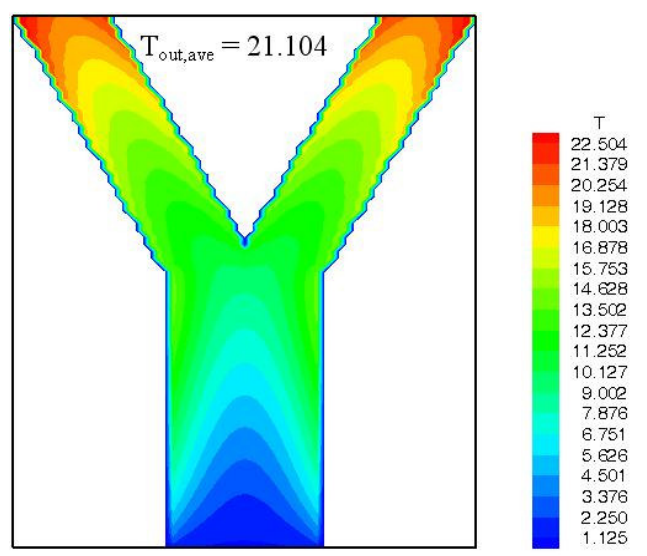

$$
\theta=30^{0}
$$

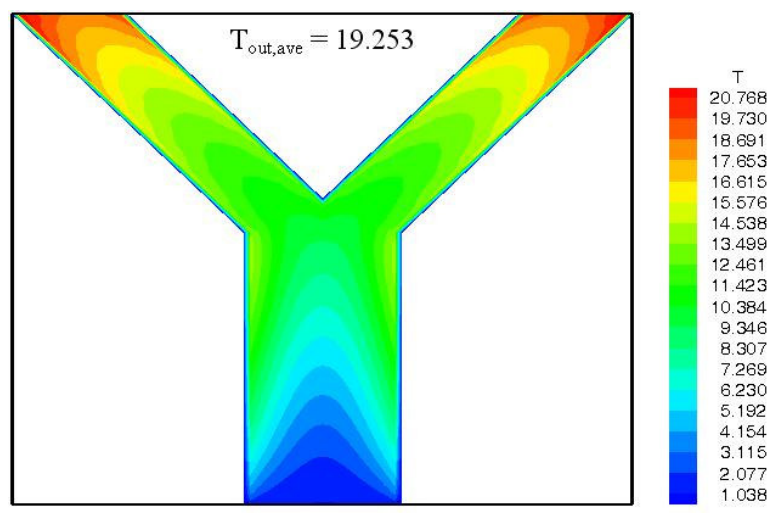

$$
\theta=45^{0}
$$

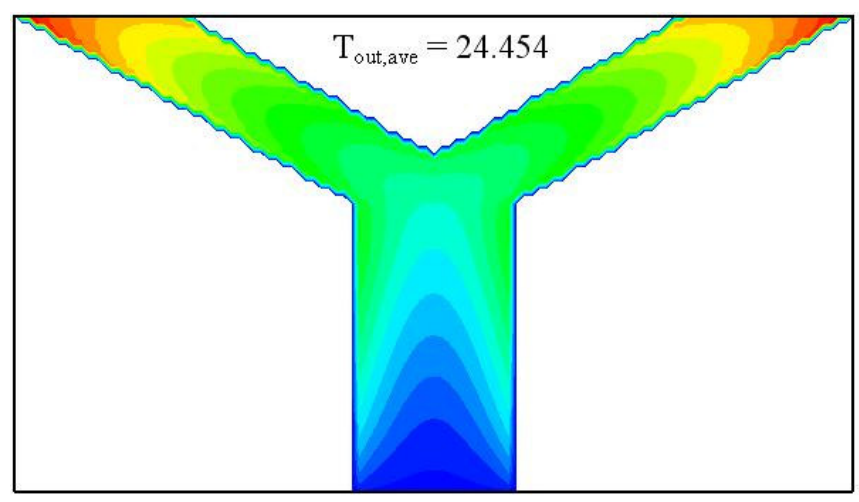

$$
\theta=60^{0}
$$

Figure 3.9 (Continued) Simulation results for $\operatorname{Re}=6$ and $q=1$ at different bifurcation angles:

(b) Temperature field. 

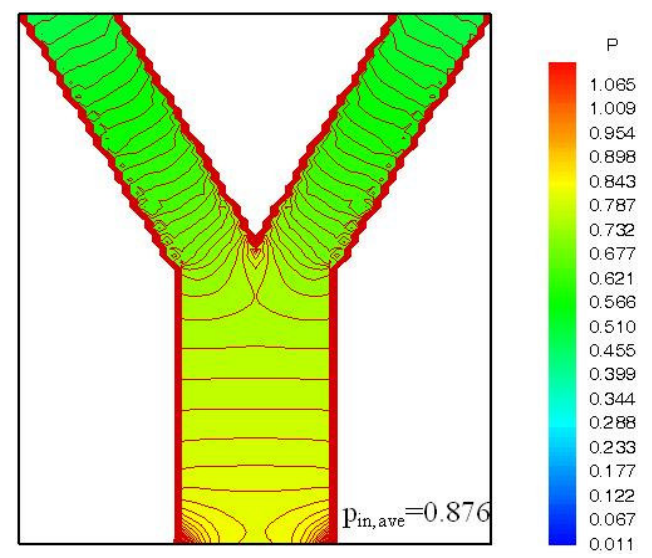

$$
\theta=30^{0}
$$

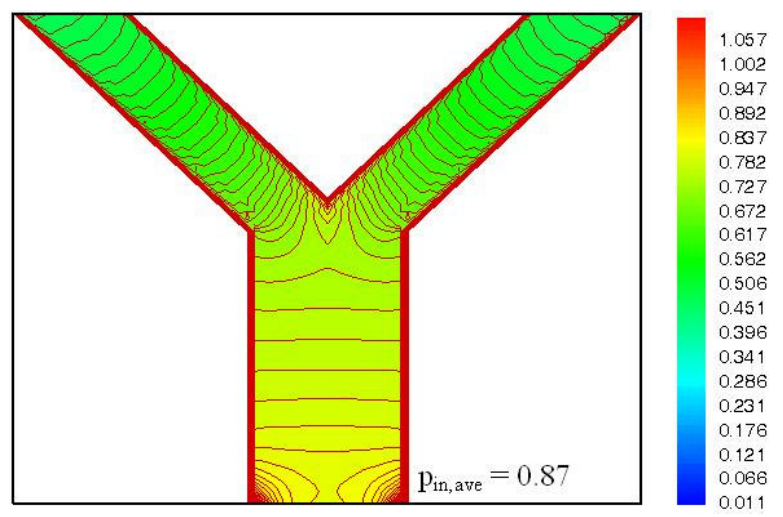

$$
\theta=45^{0}
$$

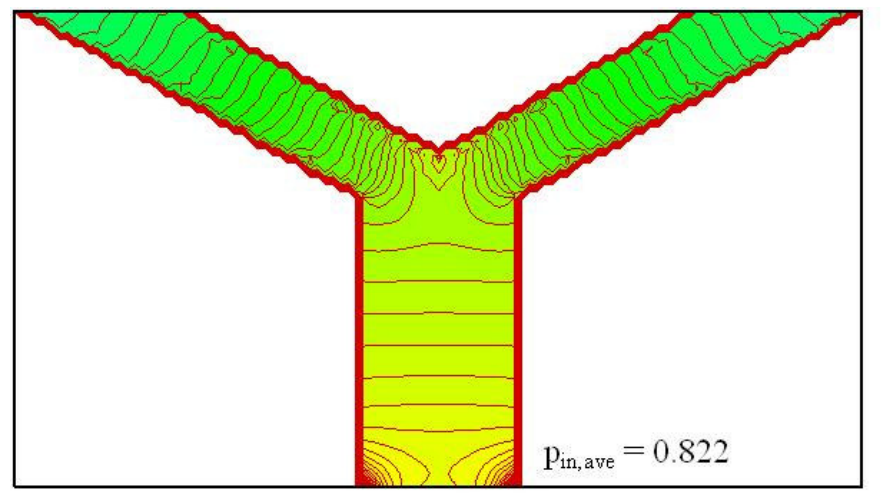

$$
\theta=60^{0}
$$

Figure 3.9 (Continued) Simulation results for $\operatorname{Re}=6$ and $q=1$ at different bifurcation angles:

(c) Pressure field. 

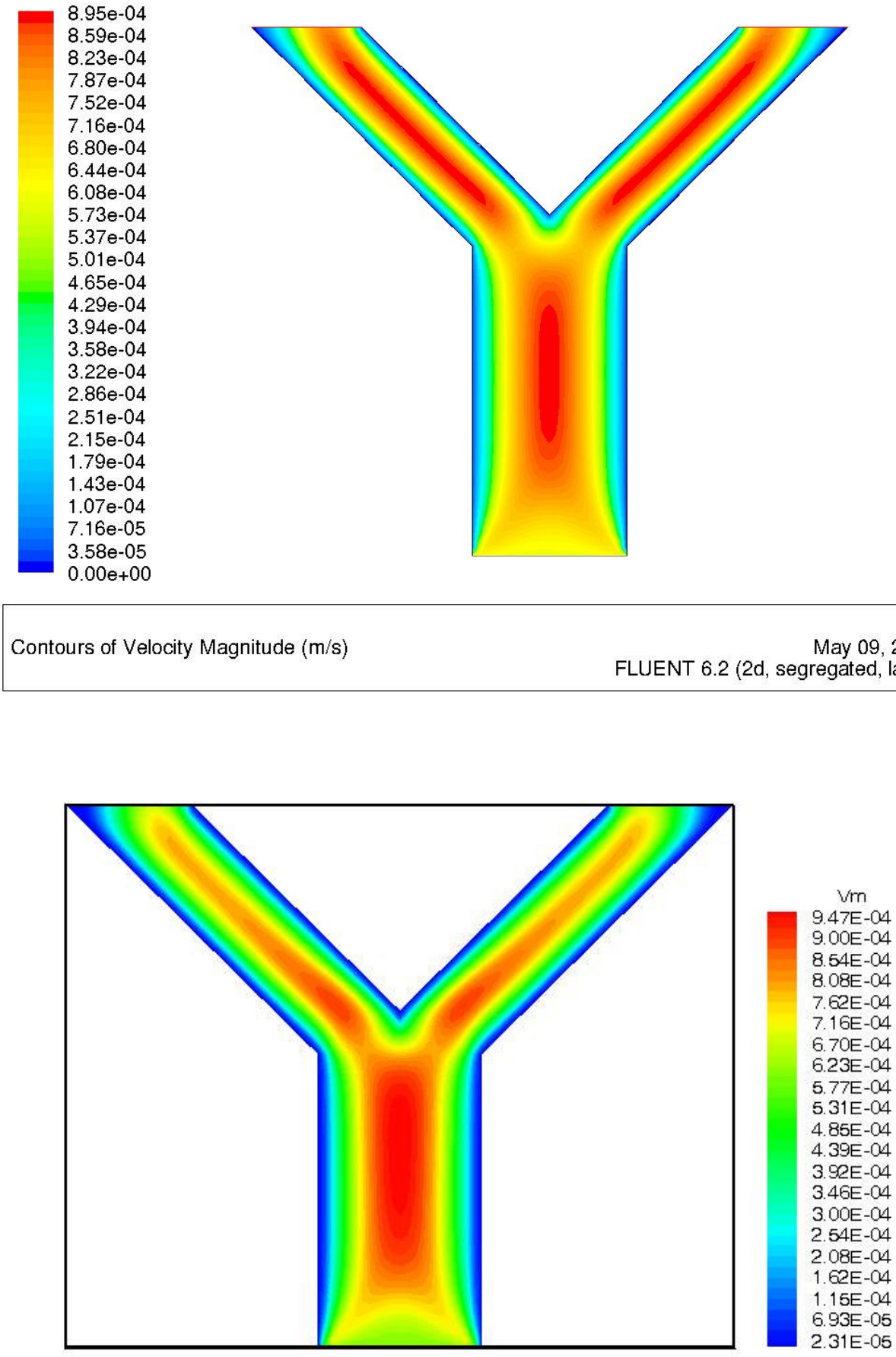

Figure 3.10 Comparison between FLUENT and our simulation results at $\theta=45^{\circ}, \operatorname{Re}=12$ and $q=1$ :

(a) Velocity magnitude. 

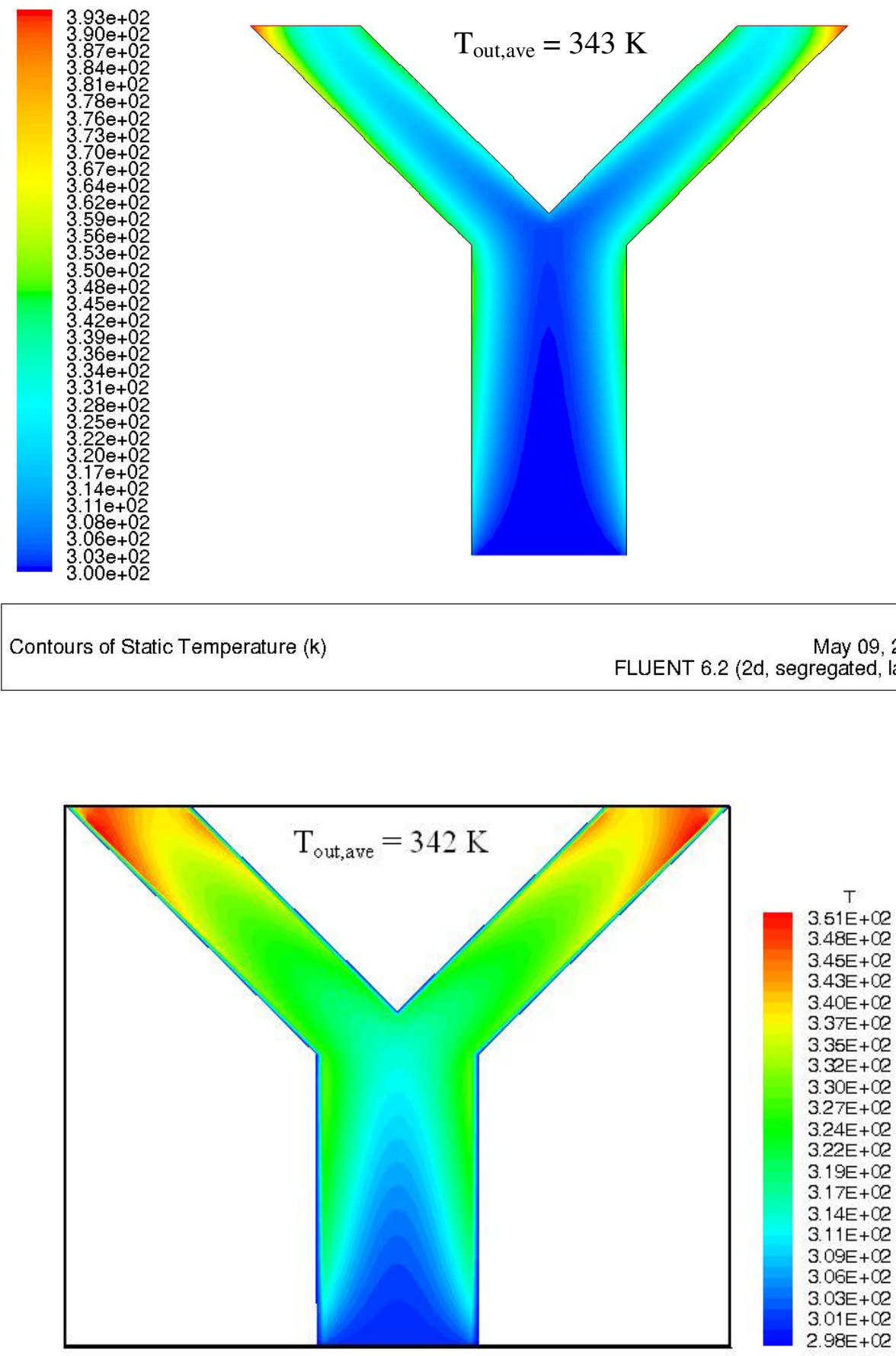

Figure 3.10 (Continued) Comparison between FLUENT and our simulation results at $\theta=45^{\circ}, \operatorname{Re}=12$ and $q=1$ :

(b) Temperature field. 

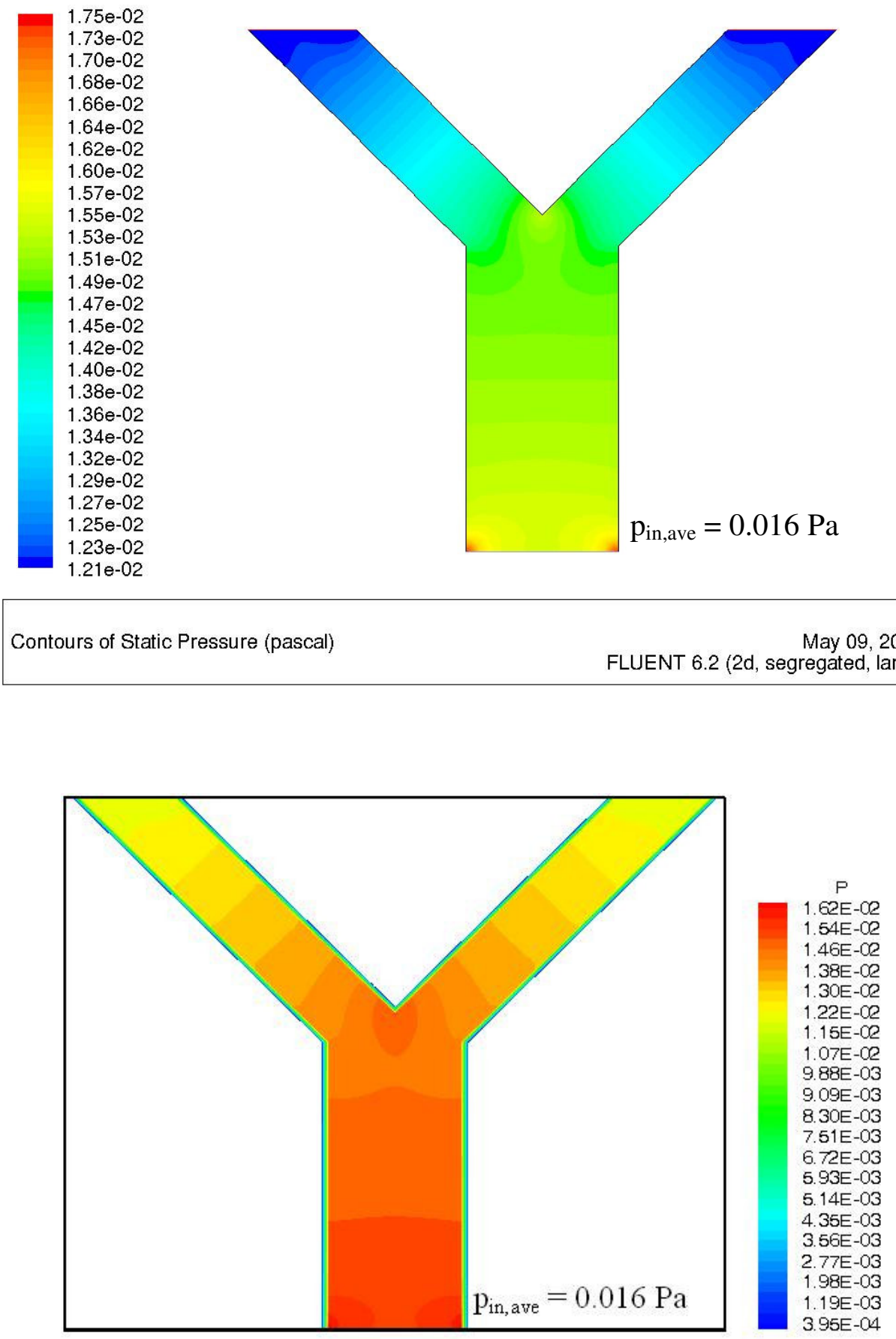

Figure 3.10 (Continued) Comparison between FLUENT and our simulation results at $\theta=45^{\circ}, \operatorname{Re}=12$ and $q=1$ :

(c) Pressure field. 


\subsection{CONCLUSIONS}

In this chapter, different LBM models and some popular boundary conditions were reviewed and formulated. By using few benchmark tests, the applicability of LBM for simulating various flow and heat transfer problems were demonstrated. The LBM can not only simulate simple flow and heat transfer problems, but also some complex systems, such as multiphase and multicomponent flows, which are difficult to handle in the conventional numerical method. The formulation of the LBM for multicomponent flow is quite simple and easy to implement compared with traditional CFD approach. This extraordinary feature makes LBM a "MUST" for particle suspension flow simulation (Succi, 2001). 


\section{CHAPTER 4}

\section{LBM FOR NANOFLUIDS}

\subsection{INTRODUCTION}

In recent years, nanofluids, consisting of nanometer-sized particles suspended in base fluids, have been proven to be effective in enhancing the performance of future energy transport systems (Eastman et al., 2004). These novel heat transfer fluids not only exhibit anomalously enhanced thermal properties, but also overcome the vulnerability of quick settling down that hinders the practical applications of conventional fluid-particle suspensions. All these characteristics make nanofluids very promising for nanotechnology-based heat transfer applications (Choi, 1995). Three fascinating features of nanofluids including anomalously high thermal conductivity at very low nanoparticle concentration, strongly temperature dependent thermal conductivity and significant increase in critical heat flux have been demonstrated by experiments (Choi, 1995; Wang et al., 1999; Vassallo et al., 2004). Such enhancement of energy transport is dependent on volume fractions and physical properties of the suspended nanoparticles and base liquids, as well as the structure of suspended nanoparticle distribution (Wang et al., 2003).

Several theoretical models also have been published to explain the possible mechanisms of heat transfer enhancement in nanofluids. Xuan and Roetzel (2000) proposed a theoretical model for the flow of nanofluids inside a tube by considering it as a single-phase fluid. Khanafer et al. (2003) studied buoyancy-driven heat transfer enhancement of nanofluids in a two-dimensional enclosure by using the finite-volume approach. Although many applications on heat transfer enhancement of nanofluids have been reported, most of them concentrate on the macroscopic phenomena occurring inside 
the nanofluids, and the methods used have been based on traditional heat transfer theory. Only few mesoscopic or microscopic methods have been applied to get insight into flow and energy transport mechanisms in nanofluids.

In reality, the nanoparticles suspended in a base fluid are surrounded by liquid molecular particles and are always under bombardment from these ambient liquid particles. During preparation of the nanofluid, some types of surface dispersants are used to get stable and even distributions of the nanoparticles, which further complicate the interactions between the solid and liquid particles. In general, the flow and energy transport enhancement of a nanofluid is controlled by several factors such as the gravitational force, Brownian force, the interfacial effect between the nanoparticles and the base liquid, and other possible external forces. Under the influence of the internal and external forces, the suspended nanoparticles are in irregular motion and in ballistic displacement even when the suspension is macroscopically stationary as a whole.

From the microscopic point of view, the existing traditional computational methods for conventional two-phase fluids can hardly reveal the inherent nature of the flow and energy transport process inside the nanofluids, which can only be studied by lattice Boltzmann method, molecular dynamics method and Monte-Carlo method. For this purpose, Xuan and Yao (2005) proposed a lattice Boltzmann scheme for nanofluids, in the light of the multicomponent LBM model proposed by Shan and Doolen (1995). The intricate interactions among the nanoparticles and the fluid particles and some external forces are included into the model. Further simulation results show that this model can successfully simulate nanofluid flow and heat transfer phenomena. 
In this chapter, the nanofluid LBM model proposed by Xuan and Yao (2005) will be used to investigate the flow and heat transfer mechanisms of nanofluids. The nanofluid in our simulation is composed of water and copper nanoparticles. The nanoparticle melting effect is included in the model by changing the heat transfer coefficient between the nanoparticle and the fluid.

\subsection{LBM FOR NANOFLUIDS}

In Xuan and Yao's (2005) LBM model for nanofluids, the base fluid and the suspended nanoparticles are considered to be two immiscible components, thus, the multicomponent LBM model proposed by Shan and Doolen (1995) was borrowed, with

the interaction forces between that base fluid and the nanoparticles calculated in a different and more complicated way. Basically, the formulation of Xuan and Yao's (2005) model is the same as the SC multicomponent model given in section.3.7. The basic equations are given as follows:

$$
\begin{aligned}
& f_{i}^{\sigma}\left(\mathbf{x}+\mathbf{e}_{i} \delta t, t+\delta t\right)=f_{i}^{\sigma}(\mathbf{x}, t)-\frac{1}{\tau_{\sigma}}\left[f_{i}^{\sigma}(\mathbf{x}, t)-f_{i}^{\sigma, e q}(\mathbf{x}, t)\right] \quad(i=0,1, \ldots, 8 ; \quad \sigma=1,2) \\
& f_{i}^{\sigma, e q}=w_{i} \rho_{\sigma}\left[1+\frac{3}{c^{2}} \mathbf{e}_{i} \cdot \mathbf{u}_{\sigma}^{e q}+\frac{9}{2 c^{4}}\left(\mathbf{e}_{i} \cdot \mathbf{u}_{\sigma}^{e q}\right)^{2}-\frac{3}{2 c^{2}}\left(\mathbf{u}_{\sigma}^{e q}\right)^{2}\right] \\
& \rho_{\sigma}=m_{\sigma} \sum_{i=0}^{8} f_{i} \\
& \rho_{\sigma} \mathbf{u}_{\sigma}^{e q}=\rho_{\sigma} \mathbf{u}^{\prime}+\tau_{\sigma} \mathbf{F}_{\text {total }, \sigma} \\
& \mathbf{u}^{\prime}=\frac{\sum_{\sigma=1}^{2}\left(\frac{m_{\sigma}}{\tau_{\sigma}} \sum_{i=0}^{8} f_{i}^{\sigma} \cdot \mathbf{e}_{i}\right)}{\sum_{\sigma=1}^{2} \frac{\rho_{\sigma}}{\tau_{\sigma}}}
\end{aligned}
$$




$$
v_{\sigma}=c_{s}^{2} \delta t\left(\tau_{\sigma}-\frac{1}{2}\right)
$$

\subsection{TLBM FOR NANOFLUIDS}

The same passive-scalar based TLBM model given in section 3.6 is also used for nanofluid simulation as follows:

$$
\begin{aligned}
& g_{i}^{\sigma}\left(\mathbf{x}+\mathbf{e}_{i} \delta t, t+\delta t\right)=g_{i}^{\sigma}(\mathbf{x}, t)-\frac{1}{\tau_{T}^{\sigma}}\left[g_{i}^{\sigma}(\mathbf{x}, t)-g_{i}^{\sigma, e q}(\mathbf{x}, t)\right] \\
& g_{i}^{\sigma, e q}=w_{i} T^{\sigma}\left[1+\frac{3}{c^{2}} \mathbf{e}_{i} \cdot \mathbf{u}_{\sigma}^{e q}+\frac{9}{2 c^{4}}\left(\mathbf{e}_{i} \cdot \mathbf{u}_{\sigma}^{e q}\right)^{2}-\frac{3}{2 c^{2}}\left(\mathbf{u}_{\sigma}^{e q}\right)^{2}\right] \\
& T^{\sigma}=\sum_{i=0}^{8} g_{i}^{\sigma} \\
& \alpha^{\sigma}=c_{s}^{2} \delta t\left(\tau_{T}^{\sigma}-\frac{1}{2}\right)
\end{aligned}
$$

As for the nanofluid system consisting of a base liquid and nanoparticles, there exists heat exchange between the fluid and the nanoparticles from the microscopic point of view. To handle such energy transport, one can use the following algorithm:

1) First, the particles of both phases assume the local equilibrium temperature (i.e., the mean macroscopic temperature) after each collision

$$
\begin{aligned}
& g_{i}^{\sigma, e q}=w_{i} T\left[1+\frac{3}{c^{2}} \mathbf{e}_{i} \cdot \mathbf{u}_{\sigma}^{e q}+\frac{9}{2 c^{4}}\left(\mathbf{e}_{i} \cdot \mathbf{u}_{\sigma}^{e q}\right)^{2}-\frac{3}{2 c^{2}}\left(\mathbf{u}_{\sigma}^{e q}\right)^{2}\right] \\
& T^{\sigma}=\sum_{i=0}^{8} g_{i}^{\sigma}
\end{aligned}
$$

2) Second, an augmentation expression, Eq. (4.12), is introduced to take into account energy exchange between the two components 


$$
T_{\text {new }}^{\sigma}=T^{\sigma}+\Delta t \tau_{T}^{\sigma} \Phi^{\sigma}
$$

where,

$$
\begin{aligned}
\Phi^{\sigma} & =\frac{h_{\sigma \bar{\sigma}}\left[T^{\bar{\sigma}}-T^{\sigma}\right] s^{\sigma}}{\rho^{\sigma} c_{p}^{\sigma}} \\
s^{\sigma} & =\frac{\text { surface area of component } \sigma \text { in a lattice }}{\text { lattice volume }}
\end{aligned}
$$

where $h_{\sigma \bar{\sigma}}$ is the heat transfer coefficient between the two components, $s^{\sigma}$ is the specific surface area of component $\sigma$ inside a lattice, and $\bar{\sigma}$ corresponds to another component that is different from $\sigma$.

3) Then, the local temperature value for each component is renewed by inserting Eq. (4.12) into Eq. (4.11)

$$
\begin{aligned}
& g_{i}^{\sigma, e q}=w_{i} T_{\text {new }}^{\sigma}\left[1+\frac{3}{c^{2}} \mathbf{e}_{i} \cdot \mathbf{u}_{\sigma}^{e q}+\frac{9}{2 c^{4}}\left(\mathbf{e}_{i} \cdot \mathbf{u}_{\sigma}^{e q}\right)^{2}-\frac{3}{2 c^{2}}\left(\mathbf{u}_{\sigma}^{e q}\right)^{2}\right] \\
& T^{\sigma}=\sum_{i=0}^{8} g_{i}^{\sigma}
\end{aligned}
$$

4) Finally, the mean temperature of the two components is obtained as

$$
T=\frac{\sum_{\sigma} \rho^{\sigma} c_{p}^{\sigma} T^{\sigma}}{\sum_{\sigma} \rho^{\sigma} c_{p}^{\sigma}}
$$

Eq. (4.16) is needed by Eq. (4.11) for the next time step.

\subsection{FORCE EVALUATION OF LBM FOR NANOFLUIDS}

In order to simulate the nanofluid by LBM, one must first analyze the dynamic factors affecting the flow. Because of the interparticle potentials and other forces on the nanoparticles, the nanofluid behaves differently from the pure liquid from the mesoscopic 
point of view and is of higher efficiency in energy transport with better stabilization compared with the common solid-liquid mixture. In the case of no other active external fields, the nanoparticles are under influence of the buoyancy and gravitational force, the Brownian force, the drag force and the dispersion force resulting from the repulsive potential. If the Brownian force is dominant, the nanoparticles may tend to aggregate and form clusters accompanying random motion of the nanoparticles. If the repulsive force among the nanoparticles is dominant, the formed clusters may be broken up and the particle aggregation process is suppressed. The effect of gravitational force is to sediment, and the clusters with larger sizes experience somewhat rapid sedimentation.

Because the nanofluid is a type of colloidal suspension, the theory of colloids (Russel et al., 1989) can be applied to describe the dynamics of the suspended nanoparticles. The forces acting on each nanoparticle can be expressed as the vector sum of the buoyancy and gravitational force, the Brownian force, the drag force and the interaction potential.

\subsubsection{The buoyancy and gravitational force}

For the buoyancy and gravity force:

$$
F_{G}=-\frac{4}{3} \pi r_{p}^{3} g\left(\rho_{f}-\rho_{p}\right)
$$

where $r_{p}$ is the radius of the suspended nanoparticle, $\rho_{f}$ and $\rho_{p}$ are the mass density of the base fluid and the nanoparticle, respectively.

\subsubsection{The drag force}

For the drag force:

$$
F_{D}=-6 \pi \mu_{f} r_{p}\left(u_{f}-u_{p}\right)
$$


where $\mu_{f}$ is the dynamic viscosity of the base fluid, $u_{f}$ and $u_{p}$ are the velocity of the base fluid and the particle, respectively.

\subsubsection{The Brownian force}

The Brownian force arises from the rapid thermal fluctuation and is a stochastic force. It represents the net effect of collisions or bombardments of the ambient liquid molecules. It obeys the Gaussian white noise distribution, i.e., it has the following correlation relations:

$$
\begin{aligned}
& \left\langle F_{B}(t)\right\rangle=0 \\
& \left\langle F_{B, i}(t) F_{B, j}\left(t^{\prime}\right)\right\rangle=C \delta_{i j} \delta\left(t-t^{\prime}\right)
\end{aligned}
$$

where $i$ and $j$ designate the Cartesian components, $\delta\left(t-t^{\prime}\right)$ is the Dirac $\delta$ function which represents that the Brownian force is instantaneous and uncorrelated for different times, and the sign \langle\rangle indicates the mean value over a period.

According to the energy balance principle, the coefficient $C$ in Eq. (4.20) is given as

$$
C=2 \gamma k_{B} T
$$

where $\gamma=6 \pi \mu_{f} r_{p}$ is the friction coefficient, $k_{B}$ is the Boltzmann constant, and $T$ is the mean temperature of the nanofluid.

In the numerical simulation, the stochastic Brownian force can be simulated by the Gaussian white noise as (Li and Ahmadi, 1992):

$$
\begin{aligned}
F_{B} & =G_{i} \sqrt{C / \Delta t} \\
& =G_{i} \sqrt{12 \pi \mu_{f} r_{p} k_{B} T / \Delta t}
\end{aligned}
$$


where $G_{i}$ is the zero mean, unit variance independent Gaussian random number.

Equation (4.22) only calculates the magnitude of the Brownian force in a given location, the direction of the Brownian force can be represented by another uniformly distributed random number.

\subsubsection{The interaction potential}

The interaction potential between the nearest-neighbor nanoparticles can be expressed as (Russel et al., 1989)

$$
V_{I}=-\frac{1}{6} A\left(\frac{2 r_{p}^{2}}{r^{2}-4 r_{p}^{2}}+\frac{2 r_{p}^{2}}{r^{2}}+\ln \frac{r^{2}-4 r_{p}^{2}}{r^{2}}\right)
$$

where $r$ is the center-to-center distance between the particles, and $A$ is the Hamaker constant which accounts for the material properties independent of the geometrical shape.

For all the nanoparticles within the adjacent lattices in the D2Q9 model, the force caused by the interaction potential is

$$
F_{I}=\sum_{i=1}^{8} N_{i} \frac{\partial V_{I}}{\partial r_{i}}
$$

where $N_{i}$ is the number of nanoparticles within the adjacent lattice.

All these forces control the displacement of the suspended nanoparticles and the morphology of the nanofluid. The vector sum of the total forces acting on the nanoparticles per unit lattice volume is

$$
F_{\text {total }, p}=\frac{N}{V}\left(F_{G}+F_{D}+F_{B}+F_{I}\right)
$$

where $N$ is the number of nanoparticles in a given lattice and $V$ is the lattice volume. 
On the other hand, the total forces acting on the fluid particles in a given lattice can be expressed as

$$
F_{\text {total }, f}=-\frac{N}{V}\left(F_{B}+F_{D}\right)
$$

\subsection{SIMULATION RESULTS}

In this section, the above numerical method is employed to investigate the flow and heat transfer enhancement effect of nanofluids.

\subsubsection{Problem description}

A microchannel of $L=0.5 \mathrm{~mm}$ in length and $D=0.1 \mathrm{~mm}$ in width is used in our simulation. The nanofluid here is composed of water as the base liquid and copper with nominal radius of $r_{p}=20 \mathrm{~nm}$ as the nanoparticles. By using D2Q9 square lattice, the domain is discretized into $100 \times 20$.

For the flow boundary conditions: the inlet is constant velocity and the exit has zero velocity gradient; the non-slip bounce-back boundary conditions are applied to the top and bottom walls. For the temperature boundary conditions: the inlet is constant temperature and the exit is adiabatic; constant heat flux is applied to the top and bottom walls. At the initial state, the nanoparticles are evenly suspended in water and the nanofluid temperature is set to $300 \mathrm{~K}$.

All the property and dimensional parameters are transferred to lattice units before calculation based on Table 4.1 by choosing the reference parameters as: $L^{*}=5.0 \times 10^{-6} \mathrm{~m}$, $U^{*}=0.5 \mathrm{~m} / \mathrm{s}, \rho^{*}=1000 \mathrm{~kg} / \mathrm{m}^{3}, \mathrm{k}^{*}=0.6 \mathrm{~W} /(\mathrm{m} \cdot \mathrm{K})$ and $\mathrm{q}^{*}=10^{5} \mathrm{~W} / \mathrm{m}^{2}$. Because of the irregular Brownian motion of the nanoparticles, the flow is not stable. The results at 50,000 time step are considered as the output results for analysis, which is significantly 
Table 4.1 Lattice unit conversion rules

\begin{tabular}{|c|c|}
\hline Tube length & $L_{l u}=\frac{L}{L^{*}}$ \\
\hline Tube diameter & $D_{l u}=\frac{D}{L^{*}}$ \\
\hline Velocity & $U_{l u}=\frac{U}{U^{*}}$ \\
\hline Density & $\rho_{l u}=\frac{\rho}{\rho^{*}}$ \\
\hline Temperature & $T_{l u}=\frac{T-T_{i n}}{q^{*} L^{*} / k^{*}}$ \\
\hline Thermal conductivity & $k_{l u}=\frac{k}{k^{*}}$ \\
\hline Heat flux & $q_{l u}=\frac{q}{q^{*}}$ \\
\hline Kinetic viscosity & $v_{l u}=\frac{v}{U^{*} L^{*}}$ \\
\hline Thermal diffusivity & $\alpha_{l u}=\frac{\alpha}{U^{*} L^{*}}$ \\
\hline
\end{tabular}

larger than the time step for pure water $(20,000)$ to reach stable state in this given domain.

In the simulation, because the nanoparticle size is very small, the particle number at each lattice site is very large. From Eq. (4.25), this will result in a relatively large force value, and the code will overflow because of it. To solve this problem, the concept of particle cluster is introduced here to make the code numerically possible to work. The simulation results presented in this section is based on the particle cluster size selection of $r_{p}=0.01$ and $r_{f}=0.1$. The reason of choosing this is discussed in section 4.6.

\subsubsection{Simulation results}

By changing the Reynolds number, wall heat flux and the nanoparticle volume fraction, the heat transfer enhancement effect of nanofluids is analyzed and compared with pure water flow. 
Figure 4.1 shows the temperature field of the nanofluids at different nanoparticle volume fraction with $\operatorname{Re}=5, q=1$. It can be seen that compared with pure water flow, the adding of nanoaprticles to the water can significantly reduced the wall temperature because of the high thermal conductivity of the nanoparticles. Due to the Brownian motion of the suspended nanoparticles under the action of various forces, the temperature distribution of the nanofluid seems to become irregular compared to that of pure water. Figure 4.3 shows the corresponding average heat transfer coefficient between the wall and the nanofluid along the channel. It can be seen that by increase the particle volume fraction, the average heat transfer coefficient can be significantly increased.

Figure 4.2 shows the temperature field of nanofluid with $\varepsilon_{p}=3 \%, q=1$ at different Reynolds numbers. It can be seen that the nanofluid temperature will decrease with the increase of Re. The Brownian motion will only play a role in very low Reynolds number, and the Brownian motion effect will be weakened by the increase of Re. Figure 4.4 shows the corresponding average heat transfer coefficient along the channel at different Re. It shows that the average heat transfer coefficient will decrease with the increase of $\mathrm{Re}$, which is different from the pure water case. The reason for this is that because the nanoparticle is very small, its effect will be greatly reduced at higher flow speed, which results in the nanofluid temperature field similar to the pure water case.

Figure 4.5 shows the nanofluid temperature at different wall heat fluxes with $\varepsilon_{p}=3 \%, \operatorname{Re}=5$. It shows the nanofluid temperature will increase with $q$, which is obviously correct. 
T: $\begin{array}{llllllllllllllllllllllllllllllll}0.1 & 0.4 & 0.7 & 1.0 & 1.3 & 1.6 & 1.9 & 2.2 & 2.5 & 2.8 & 3.1 & 3.4 & 3.7 & 4.0 & 4.3 & 4.6 & 4.9 & 5.2 & 5.5 & 5.8 & 6.1 & 6.4 & 6.7 & 7.0 & 7.3 & 7.6\end{array}$

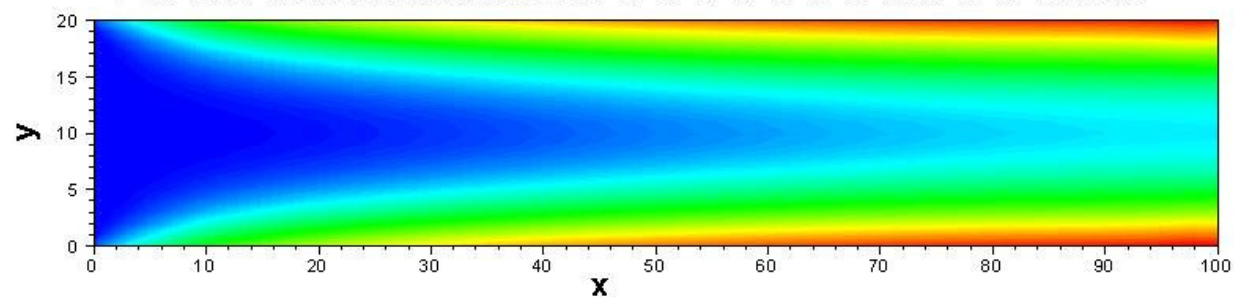

(a) pure water

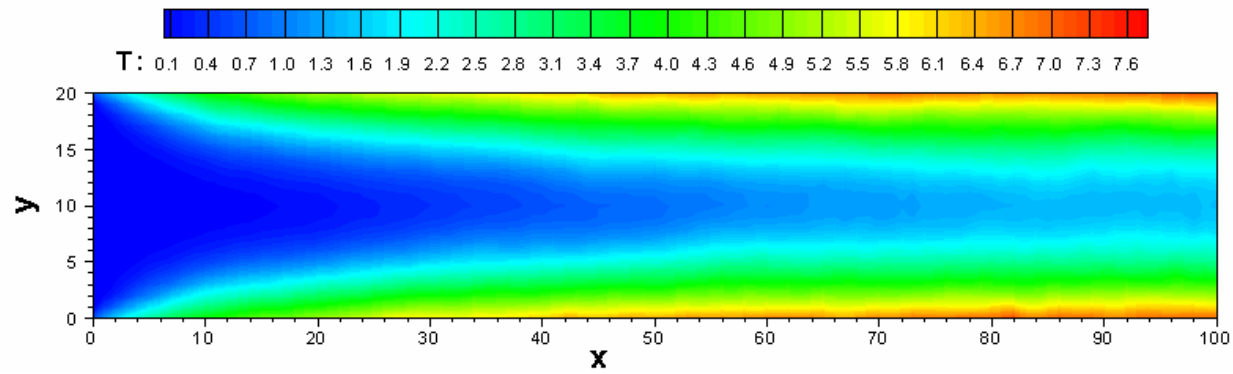

(b) $\varepsilon_{p}=1 \%$

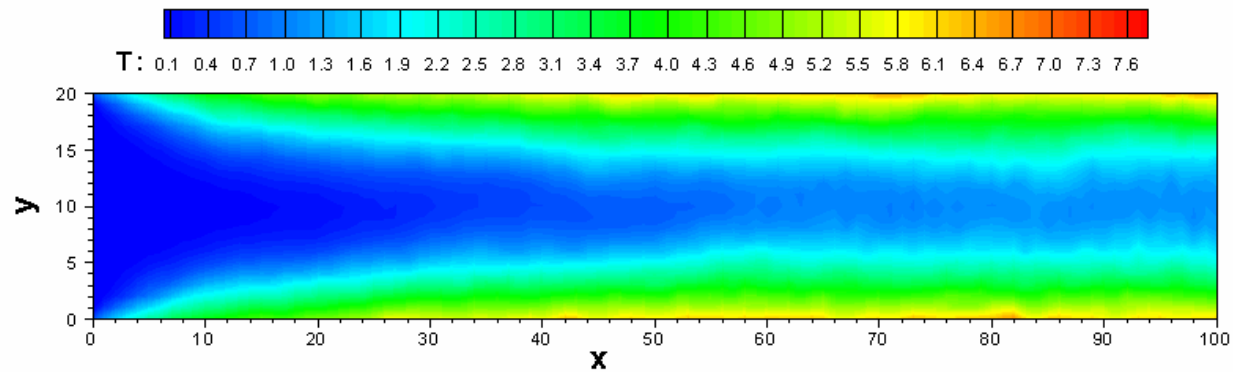

(c) $\varepsilon_{p}=2 \%$

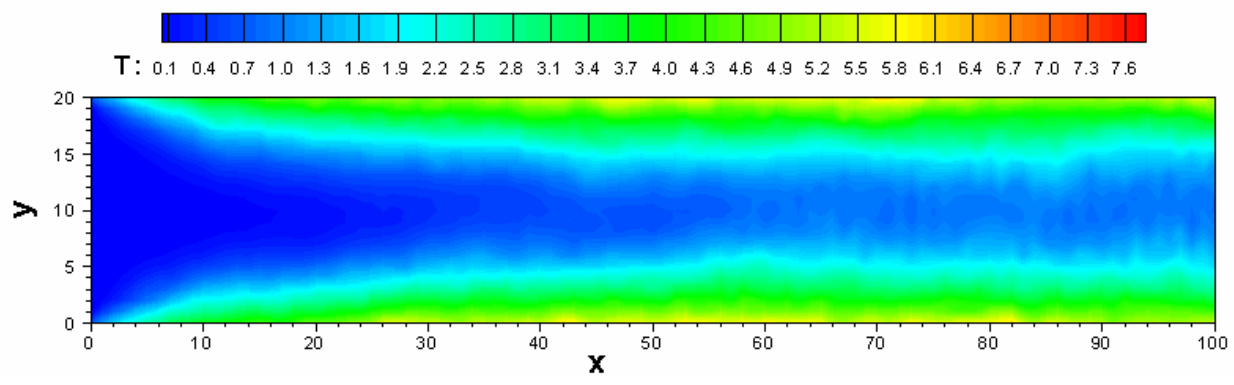

(d) $\varepsilon_{p}=3 \%$

Figure 4.1 Temperature filed of nanofluid at different $\varepsilon_{p}$ with $\operatorname{Re}=5, q=1$. 


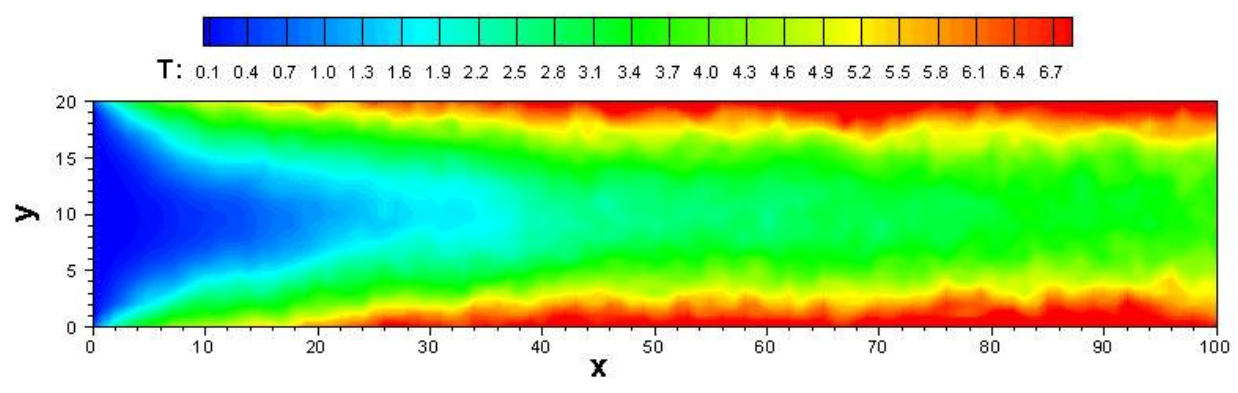

(a) $\mathrm{Re}=2$

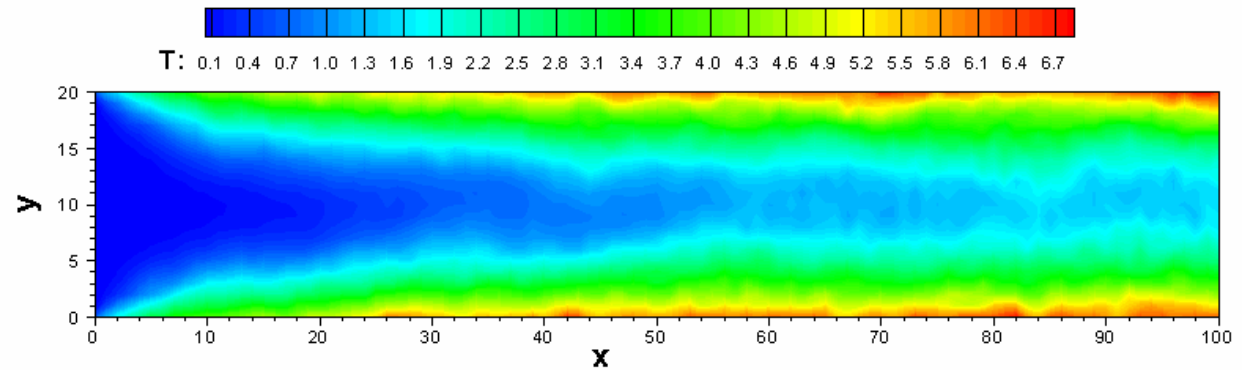

(b) $\operatorname{Re}=4$

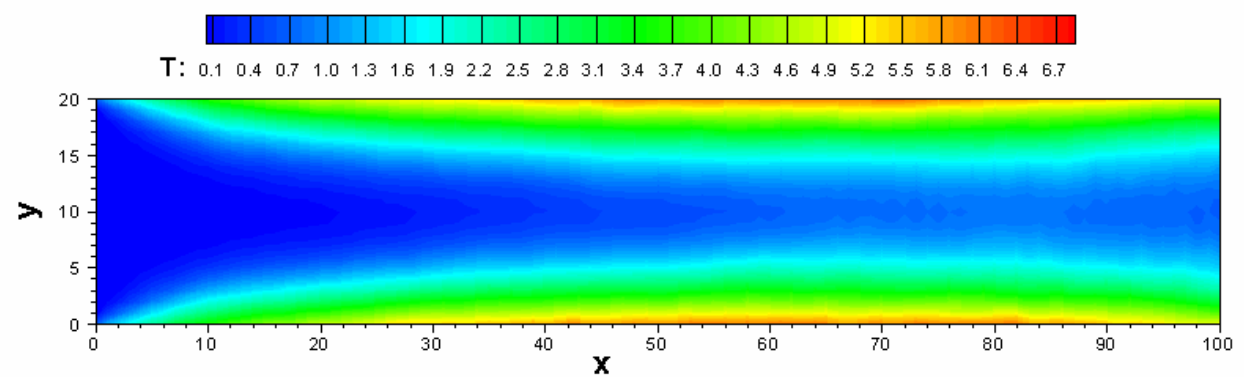

(c) $\operatorname{Re}=6$

Figure 4.2 Temperature field of nanofluid at different $\operatorname{Re}$ with $\varepsilon_{p}=3 \%, q=1$. 


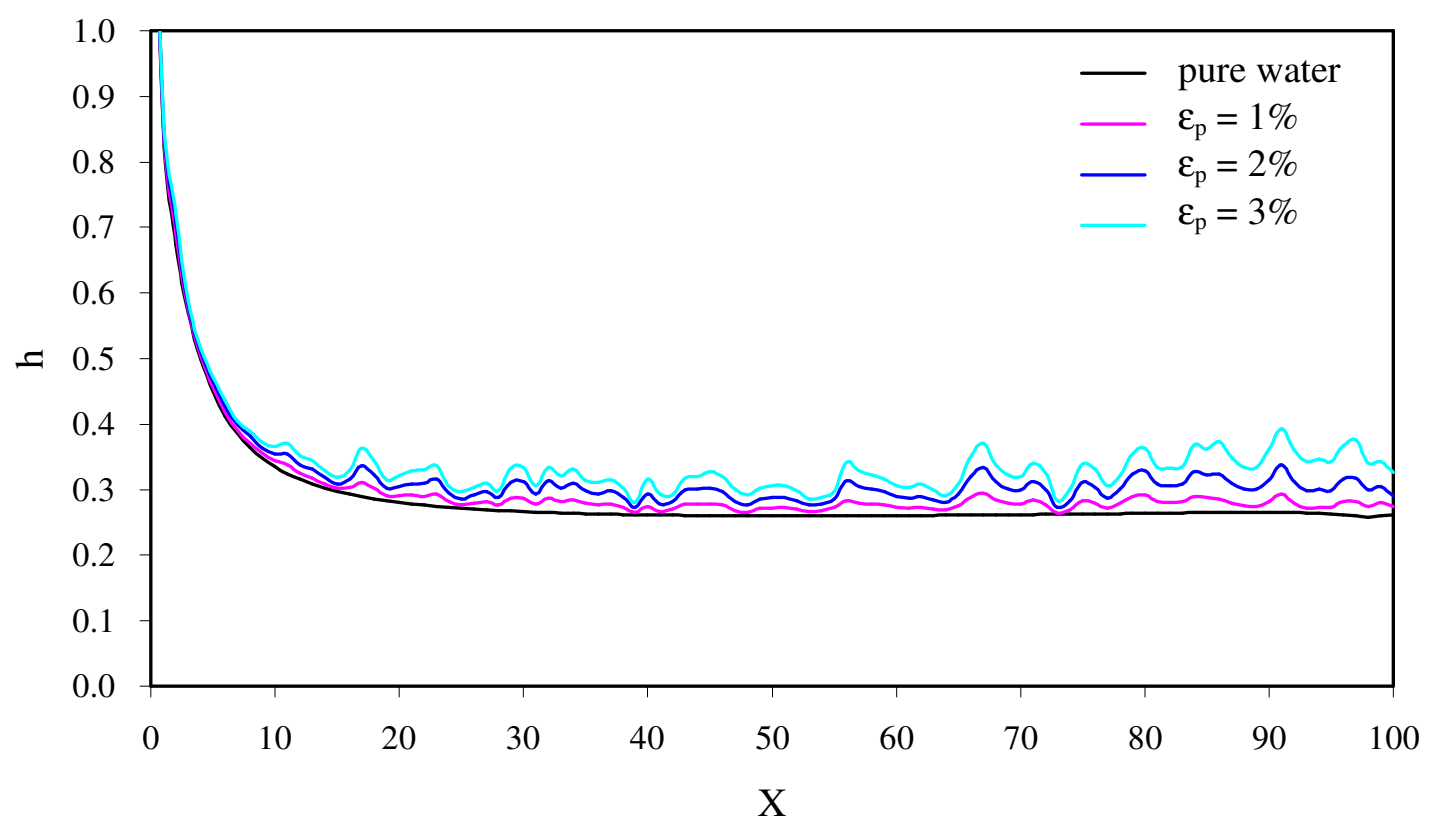

Figure 4.3 Average heat transfer coefficient of nanofluid along the channel at different $\varepsilon_{p}$ with $\operatorname{Re}=5, q=1$.

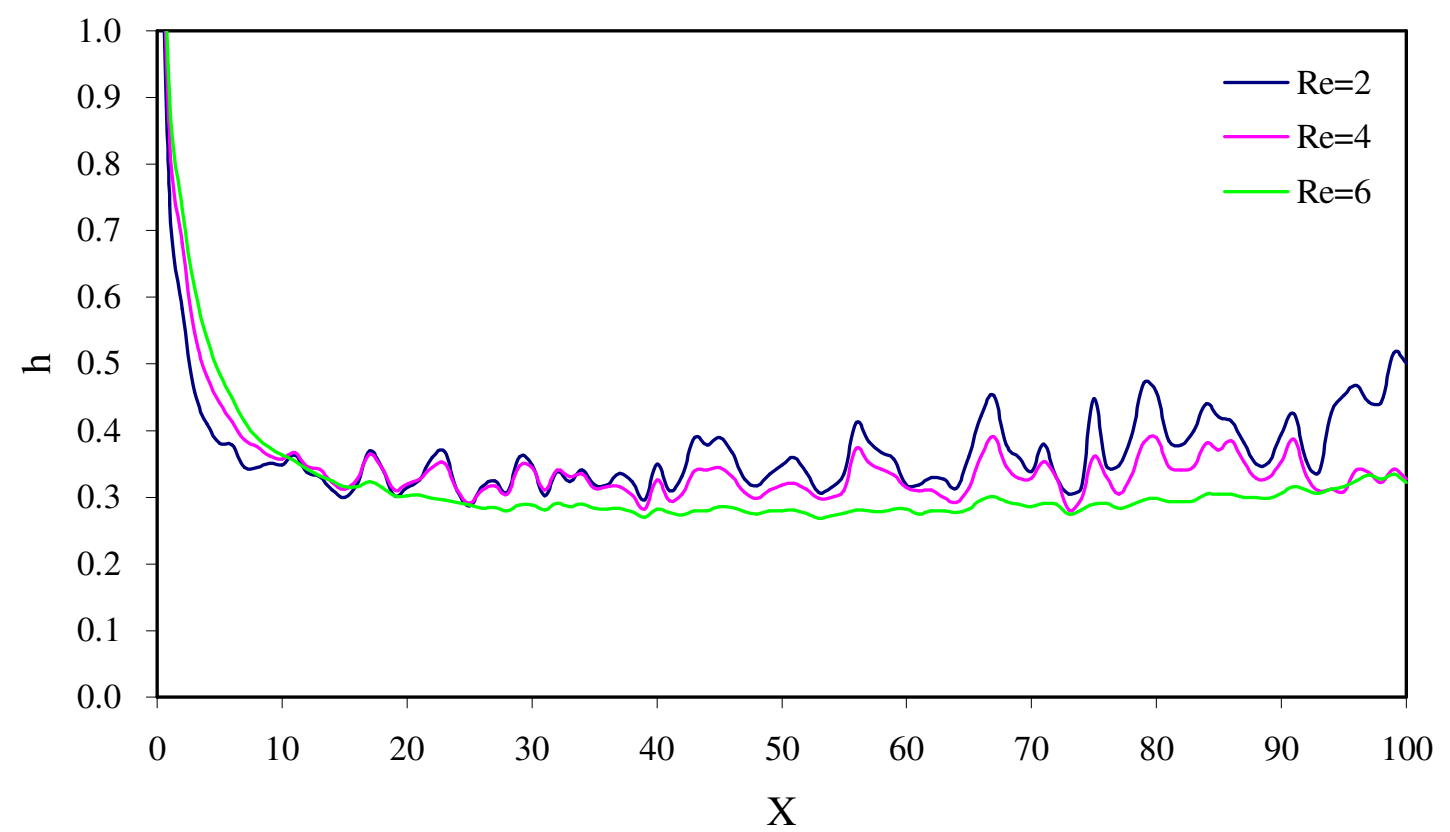

Figure 4.4 Average heat transfer coefficient of nanofluid along the channel at different $\operatorname{Re}$ with $\varepsilon_{p}=3 \%, q=1$. 


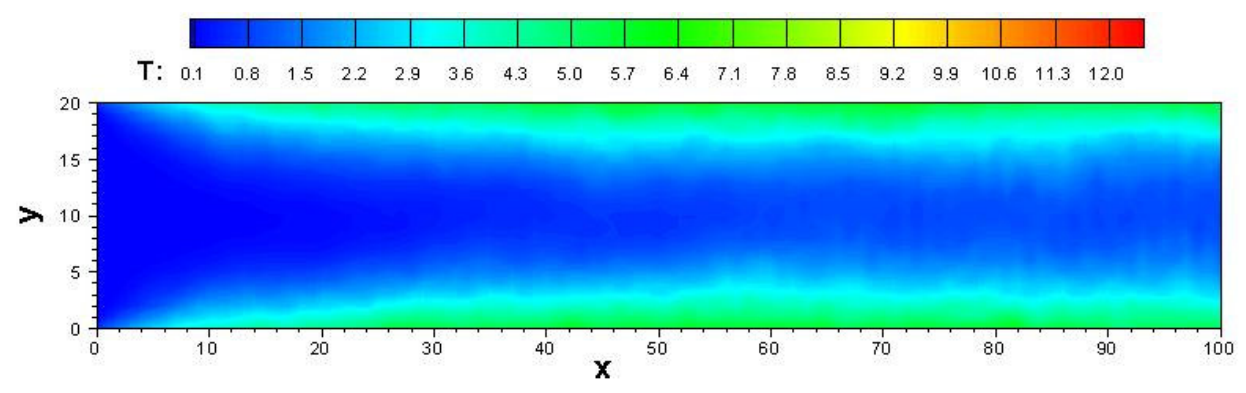

(a) $q=1$

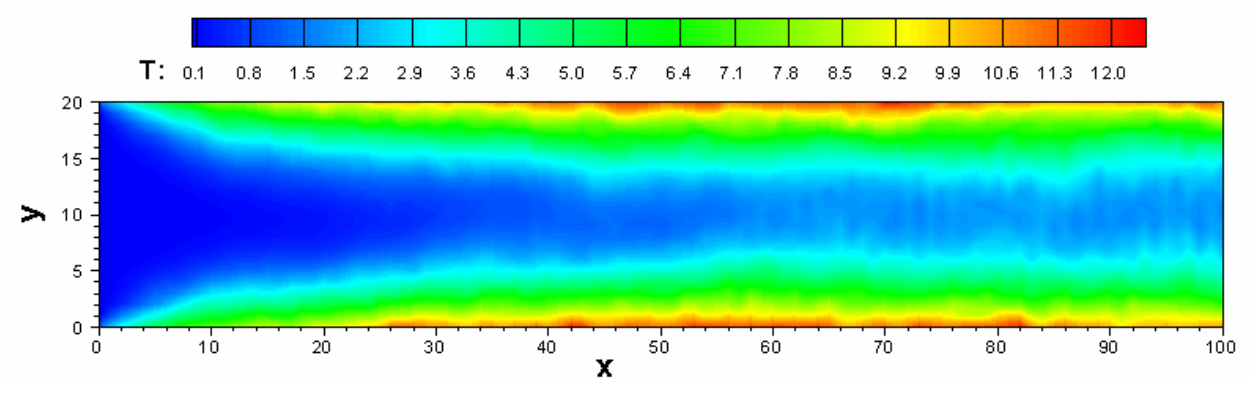

(b) $q=2$

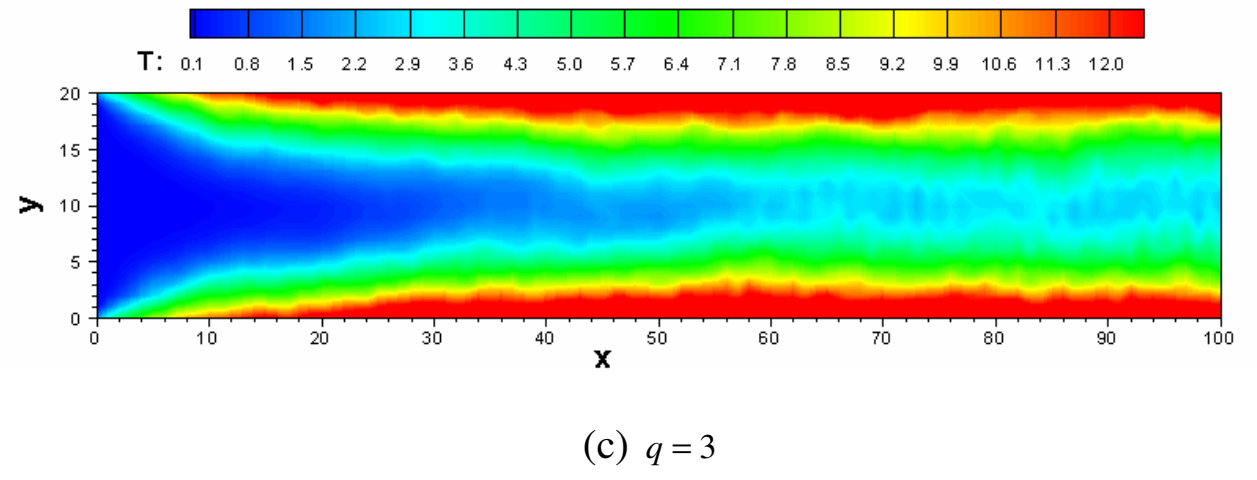

Figure 4.5 Temperature field of nanofluid along the channel at different $q$ with $\varepsilon_{p}=3 \%, \operatorname{Re}=5$. 


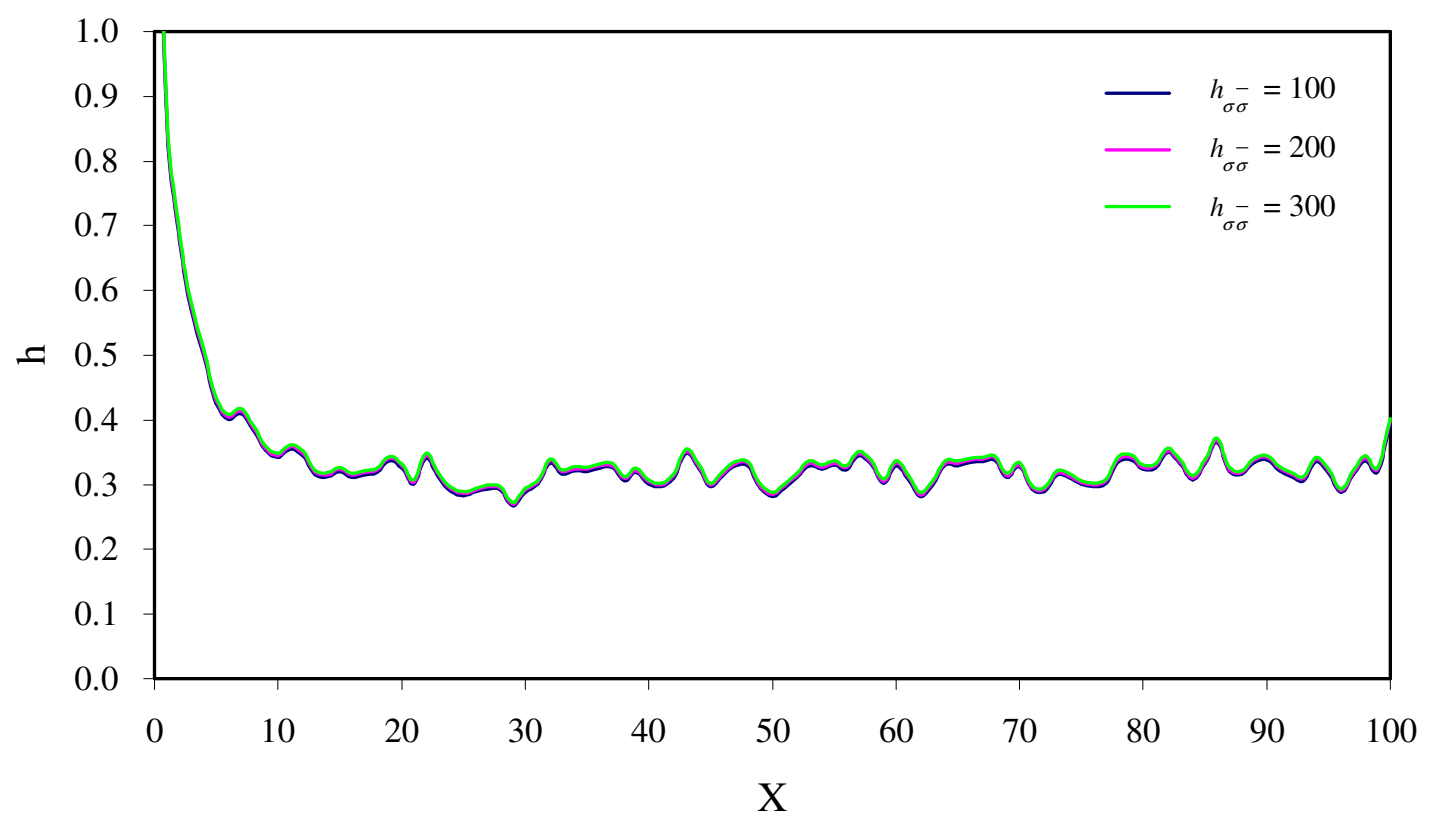

Figure 4.6 Average heat transfer coefficient of nanofluid along the channel at different $h_{\sigma \bar{\sigma}}$ with $\varepsilon_{p}=3 \%, \operatorname{Re}=5, q=1$.

In the temperature model described in section 4.3, Eq. (4.13) was introduced to take into account the energy exchange between the two components. A parameter named $h_{\sigma \bar{\sigma}}$ is used in Eq. (4.13), which is the heat transfer coefficient between the two components. To find the influence of $h_{\sigma \bar{\sigma}}$ to the nanofluid heat transfer effect, several arbitrarily selected values are used in the simulation. The result is presented in Fig. 4.6. It shows the effect of $h_{\sigma \bar{\sigma}}$ is not significant, with $h_{\sigma \bar{\sigma}}$ doubled, the average heat transfer coefficient only increased a little bit.

\subsection{PARAMETRIC STUDY OF THE PARTICLE CLUSTER SIZE EFFECT}

The concept of particle cluster size is introduced here to make the code numerically feasible. The mass of each cluster is calculated based on the cluster size and particle volume fraction as 


$$
\begin{aligned}
& m_{p}=\rho_{p} \varepsilon_{p}\left(\frac{4}{3} \pi r_{p}^{3}\right) \\
& m_{f}=\rho_{f}\left(\frac{4}{3} \pi r_{f}^{3}\right)
\end{aligned}
$$

Then the particle number density at the initial state and during the iteration is

$$
\begin{aligned}
& n_{p}=\frac{\rho_{p} \varepsilon_{p}}{m_{p}} \\
& n_{f}=\frac{\rho_{f}}{m_{f}}
\end{aligned}
$$

To see the influence of particle cluster size to the simulation results, three different combinations: $r_{p}=0.001$ and $r_{f}=0.1, r_{p}=0.01$ and $r_{f}=0.1$, and $r_{p}=0.1$ and $r_{f}=0.1$, are used in our simulation under the condition of $\varepsilon_{p}=1 \%, \operatorname{Re}=5, q=1$, to find out the best one for our purpose. The results are shown in Fig. 4.7. The mean exit temperatures for these three cases are: $\bar{T}_{\text {exit }}=3.40$ for $r_{p}=0.001$ and $r_{f}=0.1, \bar{T}_{\text {exit }}=3.70$ for $r_{p}=0.01$ and $r_{f}=0.1$, and $\bar{T}_{e x i t}=3.90$ for $r_{p}=0.1$ and $r_{f}=0.1$. The selection of the particle cluster size is based on energy balance analysis. For the case of $\varepsilon_{p}=1 \%, \operatorname{Re}=5, q=1$, only $r_{p}=0.01$ and $r_{f}=0.1$ satisfies the energy balance requirement, as presented in section 4.7.2. Therefore, we use it in our simulation. The details of the energy balance are provided in the following section. 


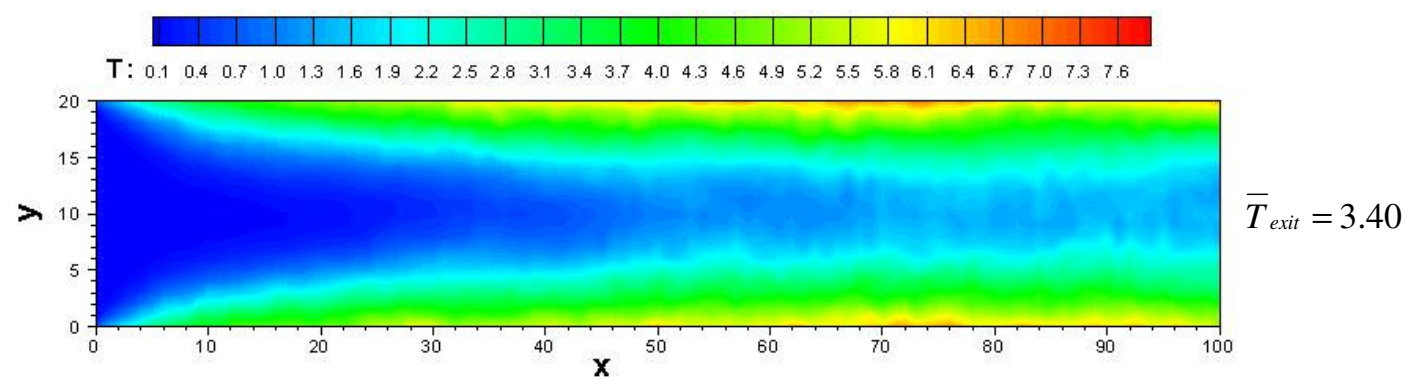

(a) $r_{p}=0.001 \& r_{f}=0.1$

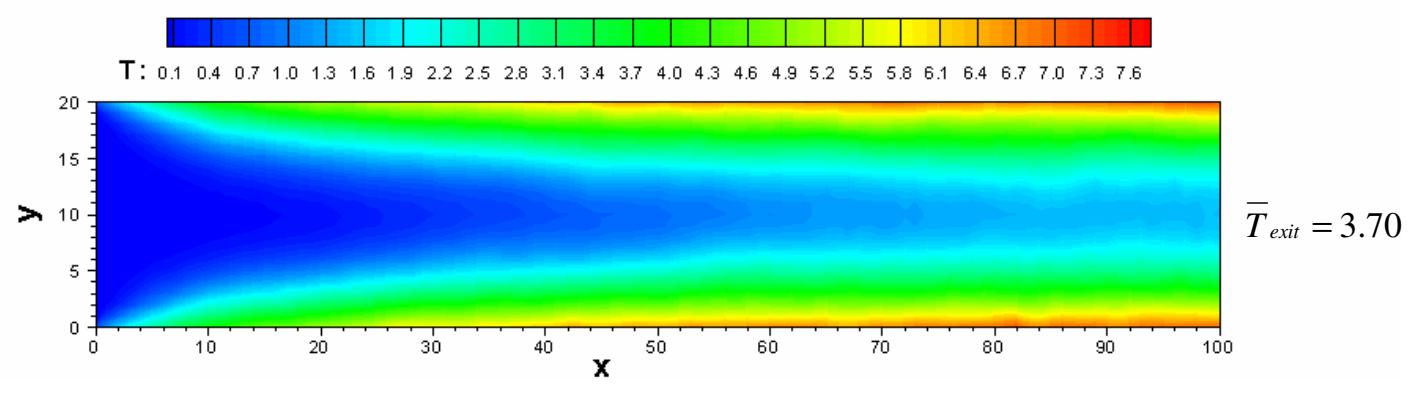

(b) $r_{p}=0.01 \& r_{f}=0.1$

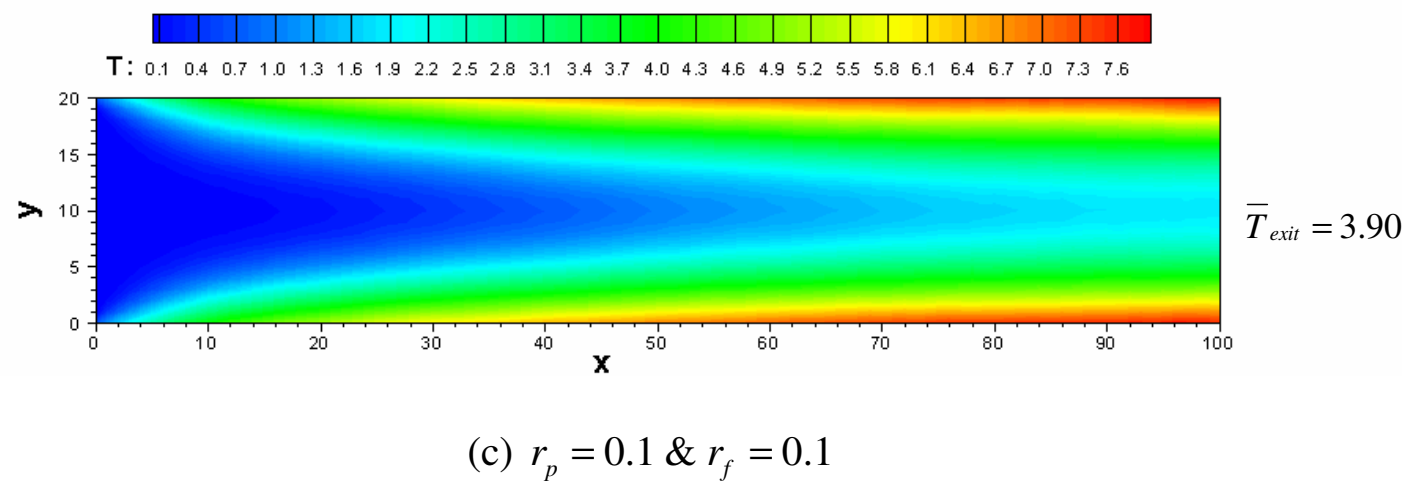

Figure 4.7 Particle cluster size effect at $\varepsilon_{p}=1 \%, \operatorname{Re}=5, q=1$. 


\subsection{NUMERICAL VALIDATION FOR NANOFLUID LBM}

Because of the lack of experimental data, experimental validation for our simulation results cannot be done at this moment. Instead, a numerical validation is provided in this section as a preliminary validation.

\subsubsection{Mass conservation}

Usually in the LBM simulation, the mass of the system is not conserved exactly. There will be mass loss/gain at each time step during the beginning stage of the simulation, which is the so-called "mass leakage" in the literature. We also observed mass leakage in our simulation. The mass leakage problem is mainly caused by the boundary condition treatment method. It can be overcome by using more complicated mass conserving boundary conditions, which is not implemented at our current research stage because of its complexity.

\subsubsection{Energy conservation}

From energy balance point of view, the total heat transferred through the wall should equal to the total heat absorbed by the nanofluid. The total heat transferred through the wall is

$$
Q 1=q_{w} \pi D L
$$

The total heat absorbed by the nanofluid is

$$
Q 2=\overline{m c_{p}} \Delta T=\bar{\rho} U \frac{\pi}{4} D^{2} \overline{c_{p}} \Delta T
$$

where, $\bar{\rho}$ and $\overline{c_{p}}$ are the mean density and heat capacity of the naofluid, which can be calculated as 


$$
\begin{aligned}
& \bar{\rho}=\varepsilon_{p} \rho_{p}+\left(1-\varepsilon_{p}\right) \rho_{f} \\
& \overline{c_{p}}=\varepsilon_{p} c_{p, p}+\left(1-\varepsilon_{p}\right) c_{p, f}
\end{aligned}
$$

Here, we take the previous case of $\varepsilon_{p}=1 \%, \operatorname{Re}=5$ and $q_{l u}=1$ as an example: the mean exit temperature from simulation is $\bar{T}_{\text {exit }, u}=3.70$, which after transfer back to physical unit, is $\bar{T}_{\text {exit }}=303.0833 \mathrm{~K}$; the other physical parameters for this case are: $L=0.5 \mathrm{~mm}, D=0.1 \mathrm{~mm}, U=0.05 \mathrm{~m} / \mathrm{s}, q_{w}=3.5 \times 10^{4} \mathrm{~W} / \mathrm{m}^{2}$, so from Eqs. (4.33) and (4.34), we can get

$$
\begin{aligned}
& \bar{\rho}=\varepsilon_{p} \rho_{p}+\left(1-\varepsilon_{p}\right) \rho_{f}=0.01 \times 8960+0.99 \times 1000=1079.6 \mathrm{~kg} / \mathrm{m}^{3} \\
& \overline{c_{p}}=\varepsilon_{p} c_{p, p}+\left(1-\varepsilon_{p}\right) c_{p, f}=0.01 \times 384.6+0.99 \times 4184=4146.0 \mathrm{~J} /(\mathrm{kg} \cdot \mathrm{K})
\end{aligned}
$$

Then from Eqs. (4.31) and (4.32), we get

$$
\begin{aligned}
Q 1 & =q_{w} \pi D L=3.5 \times 10^{4} \times \pi \times 10^{-4} \times 5 \times 10^{-4}=5.50 \times 10^{-3} \mathrm{~W} \\
Q 2 & =\bar{m} \overline{c_{p}} \Delta T=\bar{\rho} U \frac{\pi}{4} D^{2} \overline{c_{p}} \Delta T=1079.6 \times 0.05 \times \frac{\pi}{4} \times 10^{-8} \times 4146.0 \times(303.0833-300) \\
& =5.42 \times 10^{-3} \mathrm{~W}
\end{aligned}
$$

The relative difference between $Q 1$ and $Q 2$ is

$$
\frac{Q 1-Q 2}{Q 1}=\frac{5.5-5.42}{5.5}=1.45 \%
$$

The difference is relatively small, so it's acceptable. Therefore, it proves our simulation results satisfy the energy conservation.

\subsubsection{Grid independence}

To evaluate the grid independence of the present simulation, Two different meshes are used to simulate the case: $\varepsilon_{p}=1 \%, \operatorname{Re}=5$ and $q=1$. Figure 4.8 shows the 


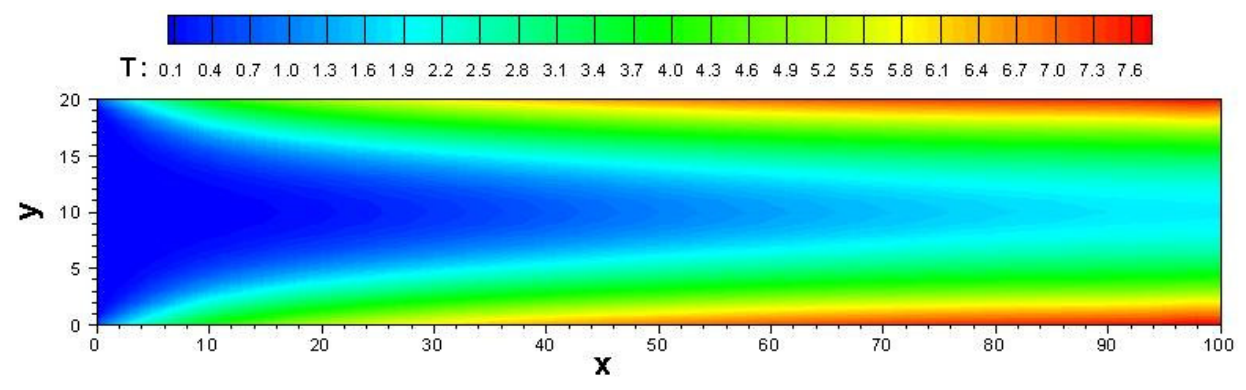

(a) $100 \times 20$

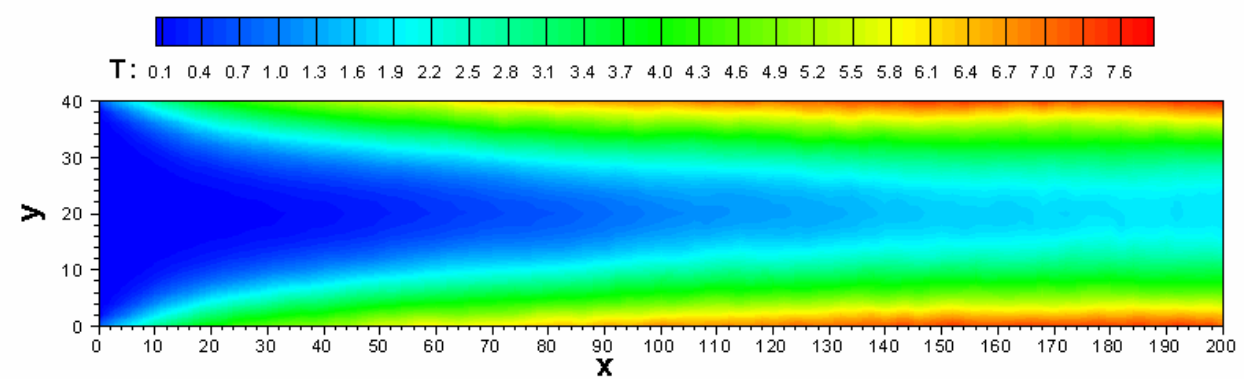

(b) $200 \times 40$

Figure 4.8 Temperature field at two different meshes.

temperature distribution at two different meshes, the results are the same. So our current model is grid independent.

\subsection{CONCLUSIONS}

In this chapter, the flow and heat transfer characteristics of nanofluid are investigated by using the two-component LBM model. By incorporating the different forces acting on the nanoparticles, the nanofluid shows significant heat transfer enhancement effect compared to single-phase flow. The concept of particle cluster size is introduced in our model, and its value is crucial to our simulation. The cluster size of 
$r_{p}=0.01$ and $r_{f}=0.1$ is chosen for the current simulation, based on the energy balance analysis. Preliminary validation is provided for our LBM model. It is found that the nanofluid will have better heat transfer enhancement effect at low Reynolds numbers and the Brownian motion effect of the nanoparticles will be weakened by the increase of flow speed. 


\section{CHAPTER 5}

\section{CONCLUSIONS AND FUTURE WORK}

\subsection{CONCLUSIONS}

In this dissertation, we have made several contributions to the study of the heat transfer enhancement mechanism of the PCM particle suspension flow by using the traditional finite volume approach, and the nanofluid flow by using the relatively new lattice Boltzmann approach. The following is a summary of our major accomplishments.

\subsubsection{Conclusions from the parametric study by using the traditional finite} volume approach

The parametric study shows that there exists an optimal relation between the channel design parameters $L$ and $D$, particle volume fraction $\varepsilon_{p}$, Reynolds number $\operatorname{Re}$, and the wall heat flux $q_{w}$.

For the normal conditions of $\operatorname{Re}=90-600$ and $\varepsilon_{p} \leq 0.25$, the following conclusions were obtained:

- For a given Reynolds number and particle volume fraction, there exists an optimal wall heat flux $q_{w, o p t}$ that will yield a maximum effectiveness factor $\varepsilon_{\text {eff, } \max }$. There also exists a maximum performance index, $P I_{\max }$, at the optimal wall heat flux $q_{w, o p t}$.

- As Re increases, $\varepsilon_{\text {eff ,max }}$ decreases. This indicates that to achieve the higher heat transfer rate by increasing $\mathrm{Re}$, the designer must be aware of the compromising of enhancement effects. 
- In general, the increase in the Reynolds number results in the decrease of heat transfer rate to pumping ratio, $Q / P$. Therefore, to best take advantage of heat transfer enhancement for micro-channel flow with PCM under laminar conditions, it is recommended that a low Reynolds number condition is maintained.

For the extreme conditions of very low Reynolds numbers $(\operatorname{Re}<50)$ and high PCM concentration $\left(\varepsilon_{p}=50 \%-70 \%\right)$ slurry flow, the following conclusions were obtained:

- The current numerical model works reasonably well for low Re and high $\varepsilon_{p}$ applications and is capable of predicting the optimal conditions for heat transfer enhancement.

- For a given Reynolds number and particle volume fraction, there exists an optimal wall heat flux that will yield a maximum effectiveness factor $\varepsilon_{\text {eff, max }}$; there also exists a maximum performance index $P I_{\max }$ at the optimal wall heat flux. This indicates that at the optimal condition, PCM flow not only can significantly enhance heat transfer, but also becomes more efficient. The physical condition for this to happen is to ensure the phase change happens within the significant (majority) portion of the heat transfer region.

- The $\varepsilon_{\text {eff, max }}-\operatorname{Re}$ relation has a peak value for $\varepsilon_{p}=0.5$ and 0.7 , which means that to achieve the best heat transfer enhancement effect with the PCM flow, one needs to find a unique match between Re and $\varepsilon_{p}$. Increasing the Reynolds number does not always enhance the heat transfer for PCM flow. 
- The correlation between $\varepsilon_{\text {eff } \text {,max }}, \varepsilon_{p}$, and Re can give a general guidance for the designer to achieve optimal design condition.

- A decrease in particle sizes tends to enhance heat transfer.

- The experimental results prove the existence of the optimal condition as predicted by the numerical simulation, and could be reasonably extrapolated from micro-channel to mini-channel applications where the continuum flow assumption holds.

Additionally, the slurry viscosity study shows that the value of particle viscosity $\mu_{p}$ has a considerable influence on the heat transfer enhancement effect of PCM suspension flow. The preliminary used value of $\mu_{p}=0.01 \mathrm{~Pa} \cdot \mathrm{s}$ is not a reasonable value under our experimental condition. Based on a through review to the available correlations for bulk viscosity of rigid spheres suspensions, a new correlation for $\mu_{p}$ is suggested through comparison with the experiment. It is also found that a particle viscosity value which is lower than water viscosity, will give a better approximation to the experimental data under the given conditions.

All the above conclusions will assist designers in making decisions that relate to the design or selection of a micro-pump suitable for micro/mini-scale heat transfer devices.

\subsubsection{Conclusions from the nanofluid simulation by LBM approach}

The flow and heat transfer characteristics of nanofluid are investigated by using the two-component LBM model. By incorporating the different forces acting on the nanoparticles, the nanofluid shows significant heat transfer enhancement effect compared to single-phase flow. The concept of particle cluster size is introduced in our model, and 
its value is chosen based on the energy balance analysis. It is found that the nanofluid will have better heat transfer enhancement effect at low Reynolds numbers and the Brownian motion effect of the nanoparticles will be weakened by the increase of flow speed.

\subsection{FUTURE WORK}

Possible future work includes:

- For the parametric study part, there is a need to investigate further the difference in the particle-level viscous friction characteristics between the solid PCM-carry fluid and liquid PCM-carry fluid interactions. A two-phase particle viscosity $\mu_{p}$ has to be defined differently in the model according to the stage of melting in the flow.

- The current nanofluid LBM model did not incorporate the nanoparticle phasechange effect yet. New solution method needs to be proposed to extend the current nanofluid LBM to incorporate the nanoparticle phase-change effect.

In this section, two possible solution methods will be proposed to incorporate the phase change effect into the current nanofluid LBM model, based on a review to the current LBM simulation of phase change phenomena.

\subsubsection{Review of the LBM simulation of solid-liquid phase-change phenomena}

Mathematical modeling of solid-liquid phase transition problems offers a challenging task to the research community, grossly attributable to the dynamic evolution of the interfaces, and the associated physical and computational complexities. To date, mathematical modeling of the phase-change problem has been based mostly on continuum approaches. As mentioned in previous chapters, LBM has been developed as a 
powerful tool for the solution of particle differential equations, and has been applied to a variety of research fields. However, there are only a few attempts to use LBM to simulate solid-liquid phase transition problems.

De Fabritiis et al. (1998) proposed a generalized mesoscopic LB model for describing flows with solid-liquid phase transitions. They used a thermal model with two types of quasi-particles for liquid and solid phases, respectively. The phase transition was represented by a chemical term, while the melting and solidification were analogous to exothermic and endothermic chemical reactions. Besides being restricted to the onedimensional case, this model contained a number of empirical parameters and assumptions which cast some doubts on its viability for more realistic studies. Miller et al. (2001) simplified and extended De Fabritiis's model by using only one type of quasiparticles, along with a phase field approach, which showed a more elegant computational capability in treating the solid-liquid phase transition. Jiaung et al. (2001) proposed an extended LB methodology for the heat conduction problem with phase-change, in conjunction with an enthalpy formulation for treatment of solid-liquid phase change aspects. In this model, the interfacial position of phase change can be determined through the liquid-phase fraction. However, apart from being two-dimensional, the model lacked from a general perspective of being extendible to simulate solid-liquid phase transition of multi-component systems. Based on Jiaung's work, Chatterjee and Chakraborty (2005, 2006) developed a generalized three-dimensional LB method for modeling conductiondominated solid-liquid phase transition problem, with the aid of a thermodynamicallyconsistent enthalpy updating scheme. Phase change phenomenon of a single-component system is computationally handled by the classical enthalpy method. A modified latent 
heat updating procedure is integrated with the lattice Boltzmann equation, for accurately predicting the liquid fraction during the continuous evolution of the solid-liquid interface. This model can also be extended to the solution of multi-component solidification problems, by judiciously modifying the latent heat updating function. Of all these models, Chatterjee and Chakraborty's model has the best potential to be used for simulating phase change particle suspension flow.

In Chapter 4, in the TLBM model we used for nanofluid simulation, we introduced Eq. (4.13) to take into account the energy exchange between the nanoparticle and the carrier fluid. A parameter, $h_{\sigma \bar{\sigma}}$, called the heat transfer coefficient between the nanoparticle and the carrier fluid, was used in Eq. (4.13). In the previous simulation, $h_{\sigma \bar{\sigma}}$ was set as constant. However, in the case of melting inside the nanoparticle, $h_{\sigma \bar{\sigma}}$ cannot be taken as a constant anymore. Therefore, if we can find a dynamic expression for $h_{\sigma \bar{\sigma}}$, it might be able to take into account the phase change effect of the nanoparticle on the heat transfer enhancement.

In this section, two solution methods for simulating PCM suspension flow with LBM will be proposed. The first method is to derive a dynamic expression for $h_{\sigma \bar{\sigma}}$ to incorporate the particle phase change effect into the current nanofluid LBM model, based on the classical Stefan melting problem. The second method is to use Chatterjee and Chakraborty's LBM model to simulate the particle phase change phenomenon and discuss its potential to be used in our future work. 


\subsubsection{Solution method 1}

The term "Stefan problem" is generally used for heat transfer problems with phasechange such as from the solid to the liquid. The typical feature of the Stefan problem is the existence of a free boundary or a moving boundary. For the melting process, if the initial temperature of the PCM, $T_{i}$, equals the melting temperature, $T_{m}$, only the temperature in the liquid phase needs to be determined. Thus, the problem is called a oneregion problem (Fig. 5.1(a)). On the other hand, if the initial temperature of the PCM, $T_{i}$, is below the melting point of the PCM, $T_{m}$, the temperature distribution of both the liquid and solid phases must be determined; this is called a two-region problem (Fig. 5.1(b)).

In this section, an equation for the heat transfer coefficient between the PCM particle and the carrier fluid will be derived, based on the two-region Stephan melting problem.

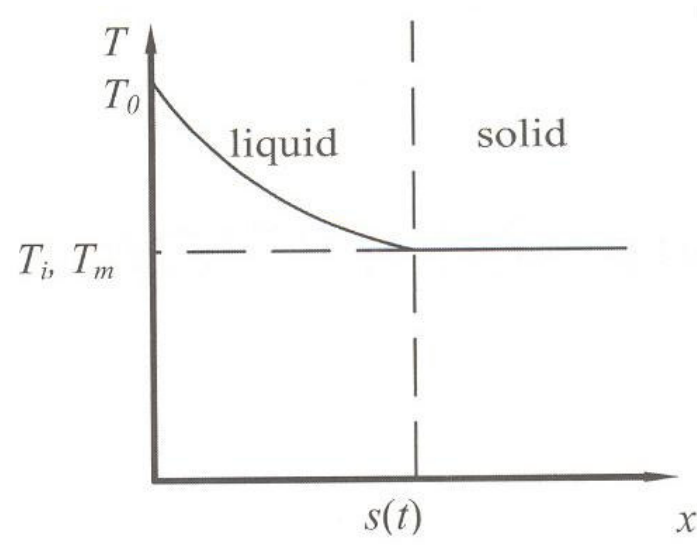

(a)

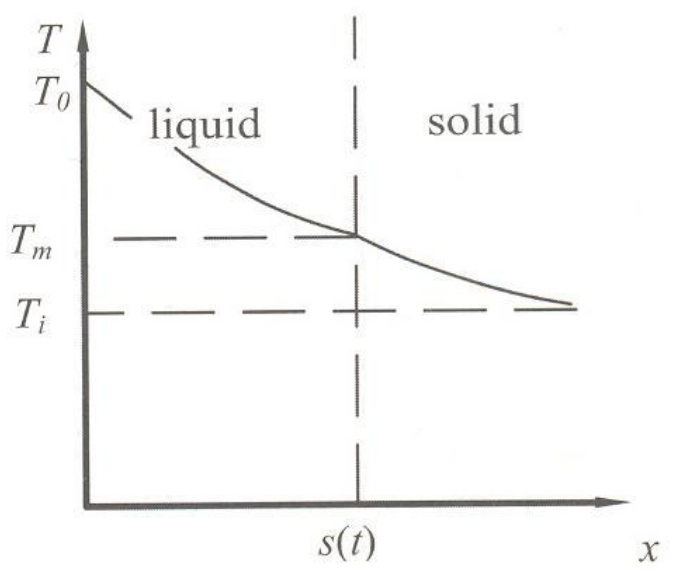

(b)

Figure 5.1 (a) One-region melting; (b) Two-region melting. (Source: Faghri and Zhang, 2006, p.422) 


\section{Governing equations of the melting problem}

The physical model of the melting problem to be investigated is shown in Fig. 5.1(b), where a solid PCM with a uniform initial temperature $T_{i}$, which is below the melting point $T_{m}$, is in a half-space $x>0$. At time $t=0$, the temperature at the boundary $x=0$ is suddenly increased to a temperature $T_{0}$, which is above the melting point of the PCM. Melting occurs from the time $t=0$. This is a two-region melting problem as the temperatures of both the liquid and solid phases are unknown and must be determined. It is assumed that the densities of the PCM for both phases are the same. Natural convection in the liquid phase is neglected, and therefore the heat transfer mechanism in both phases is pure conduction.

The temperature in the liquid phase must satisfy

$$
\begin{array}{cc}
\frac{\partial^{2} T_{1}}{\partial x^{2}}=\frac{1}{\alpha} \frac{\partial T_{1}}{\partial t} & 0<x<s(t), t>0 \\
T_{1}(x, t)=T_{0} & x=0, t>0
\end{array}
$$

where $\alpha_{1}$ is the thermal diffusivity of the liquid phase.

For the solid phases, the governing equations are

$$
\begin{array}{rl}
\frac{\partial^{2} T_{2}}{\partial x^{2}}=\frac{1}{\alpha_{2}} \frac{\partial T_{2}}{\partial t} & s(t)<x<\infty, t>0 \\
T_{2}(x, t) \rightarrow T_{i} & x \rightarrow \infty, t>0 \\
T_{2}(x, t)=T_{i} & x>0, t=0
\end{array}
$$

The boundary conditions at the interface are

$$
T_{1}(x, t)=T_{2}(x, t)=T_{m} \quad x=s(t), t>0
$$




$$
k_{1} \frac{\partial T_{1}}{\partial x}-k_{2} \frac{\partial T_{2}}{\partial x}=\rho L \frac{d s}{d t} \quad x=s(t), t>0
$$

where $k_{1}$ and $k_{2}$ are the thermal conductivity of the liquid and solid phases, respectively;

$L$ is the latent heat of the PCM.

\section{Dimensionless form of the governing equations}

The governing equations (5.1) - (5.7) can be nondimensionalized by introducing the following dimensionless variables:

$$
\left.\begin{array}{l}
\theta=\frac{T_{m}-T}{T_{m}-T_{0}} \quad \theta_{i}=\frac{T_{m}-T_{i}}{T_{m}-T_{0}} \quad X=\frac{x}{L_{0}} \quad S=\frac{s}{L_{0}} \quad \tau=\frac{\alpha_{1} t}{L_{0}{ }^{2}} \\
N_{\alpha}=\frac{\alpha_{2}}{\alpha_{1}} \quad N_{k}=\frac{k_{2}}{k_{1}} \quad \text { Ste }=\frac{c_{p, 1}\left(T_{m}-T_{0}\right)}{L}
\end{array}\right\}
$$

where $L_{0}$ is the characteristic length of the problem and can be determined by the nature of the problem or requirement of the solution procedure; Ste is the Stefan number, which represents the ratio of sensible heat to latent heat.

$$
\begin{array}{cl}
\frac{\partial^{2} \theta_{1}}{\partial X^{2}}=\frac{\partial \theta_{1}}{\partial \tau} & 0<X<S(\tau), \tau>0 \\
\theta_{1}(X, \tau)=1 & X=0, \tau>0 \\
\frac{\partial^{2} \theta_{2}}{\partial X^{2}}=\frac{1}{N_{\alpha}} \frac{\partial \theta_{2}}{\partial \tau} & S(\tau)<X<\infty, \tau>0 \\
\theta_{2}(X, \tau) \rightarrow \theta_{i} & X \rightarrow \infty, \tau>0 \\
\theta_{2}(X, \tau)=\theta_{i} & X>0, \tau=0 \\
\theta_{1}(X, \tau)=\theta_{2}(X, \tau)=0 & X(\tau), \tau>0 \\
-\frac{\partial \theta_{1}}{\partial X}+N_{k} \frac{\partial \theta_{2}}{\partial X}=\frac{1}{S t e} \frac{d S}{d \tau} & X=S(\tau), \tau>0
\end{array}
$$




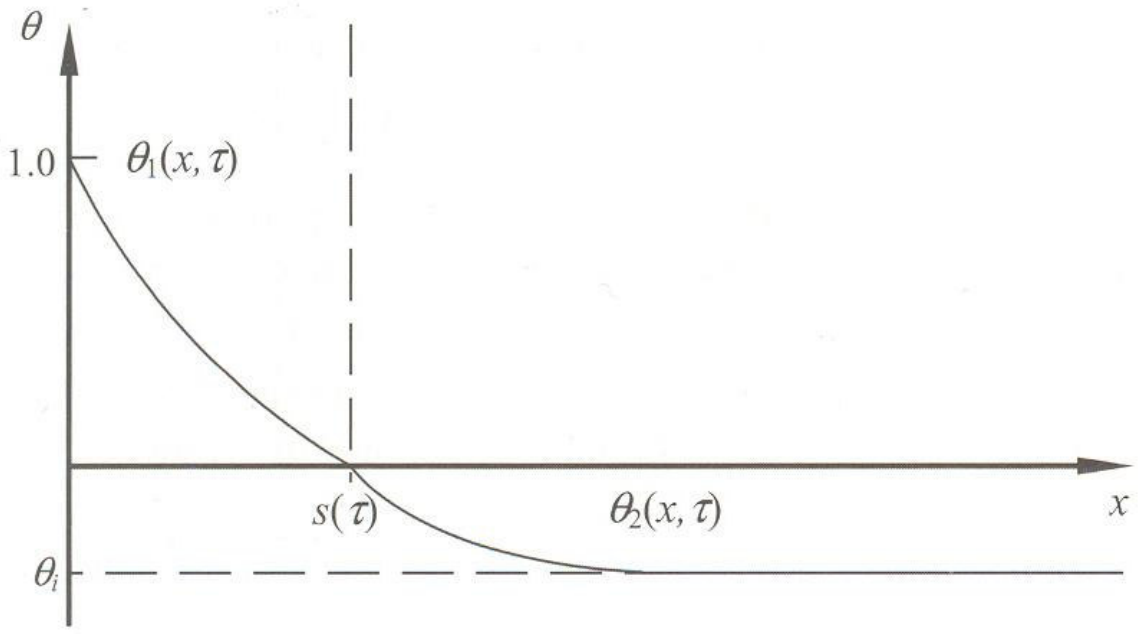

Figure 5.2 Dimensionless temperature distributions in the PCM. (Source: Faghri and Zhang, 2006, p.429)

Dimensionless temperature distribution in a PCM can be qualitatively illustrated by Fig. 5.2, with $\theta_{1}$ and $\theta_{2}$ represent the dimensionless temperature of the liquid and solid phases, respectively.

\section{Exact solution of the two-region melting problem}

The two-region problem is also called Neumann problem in the literature. Equations (5.9) - (5.15) provide the complete mathematical description of a Neumann problem.

Based on the heat conduction solution of a semi-infinite body, the temperature distribution in the PCM can be constructed as follows:

$$
\begin{aligned}
& \theta_{1}(X, \tau)=1+A \cdot \operatorname{erf}\left(\frac{X}{2 \tau^{1 / 2}}\right) \\
& \theta_{2}(X, \tau)=\theta_{i}+B \cdot \operatorname{erfc}\left(\frac{X}{2\left(N_{\alpha} \tau\right)^{1 / 2}}\right)
\end{aligned}
$$


where $A$ and $B$ are unspecified constants, and $\operatorname{erfc}$ is the complementary error function, defined as

$$
\operatorname{erfc}(z)=1-\operatorname{erf}(z)
$$

It should be noted that Eqs. (5.16) and (5.17) satisfy Eqs. (5.9) - (5.13). The constants $A$ and $B$ can be determined by using boundary condition (5.14), i.e.,

$$
\begin{aligned}
& 1+A \cdot \operatorname{erf}\left(\frac{S}{2 \tau^{1 / 2}}\right)=0 \\
& \theta_{i}+B \cdot \operatorname{erfc}\left(\frac{S}{2\left(N_{\alpha} \tau\right)^{1 / 2}}\right)=0
\end{aligned}
$$

Since $A$ and $B$ are constants, $\frac{S}{2 \tau^{1 / 2}}$ must also be a constant in order for Eqs. (5.19) and (5.20) to be satisfied. This constant can be represented by $\lambda$, so

$$
\lambda=\frac{S}{2 \tau^{1 / 2}}
$$

Thus, the constant $A$ and $B$ can be determined as

$$
\begin{aligned}
& A=-\frac{1}{\operatorname{erf}(\lambda)} \\
& B=-\frac{\theta_{i}}{\operatorname{erfc}\left(\lambda / N_{\alpha}^{1 / 2}\right)}
\end{aligned}
$$

Substituting Eqs. (5.22) and (5.23) into Eqs. (5.16) and (5.17), the temperature distributions in both phases are determined as follows:

$$
\theta_{1}(X, \tau)=1-\frac{\operatorname{erf}\left(\frac{X}{2 \tau^{1 / 2}}\right)}{\operatorname{erf}(\lambda)}
$$




$$
\theta_{2}(X, \tau)=\theta_{i}\left[1-\frac{\operatorname{erf}\left(\frac{X}{2\left(N_{\alpha} \tau\right)^{1 / 2}}\right)}{\operatorname{erf}\left(\frac{\lambda}{N_{\alpha}^{1 / 2}}\right)}\right]
$$

Substituting Eqs. (5.24), (5.25), and (5.21) into Eq. (5.15), the following equation is obtained for the constant $\lambda$ :

$$
\frac{e^{-\lambda^{2}}}{\operatorname{erf}(\lambda)}+\frac{N_{k} \theta_{i}}{N_{\alpha}^{1 / 2}} \frac{e^{-\lambda / N_{\alpha}}}{\operatorname{erfc}\left(\lambda / N_{\alpha}^{1 / 2}\right)}=\lambda \frac{\sqrt{\pi}}{\text { Ste }}
$$

Eq. (5.26) can be solved for $\lambda$ by using an iterative method. Once $\lambda$ is obtained, the temperature distributions $\theta_{1}(X, \tau)$ and $\theta_{2}(X, \tau)$, and the location of the solid-liquid interface $S(\tau)$ can be obtained from Eqs. (5.24), (5.25), and (5.21), respectively.

\section{Heat transfer coefficient between the melting PCM particle and the carrier fluid}

Our purpose is trying to find the heat transfer coefficient between the melting PCM particle and the carrier fluid. Let's assume the carrier fluid temperature is $T_{f}$ and the mean temperature of the melting PCM particle is $\overline{T_{p}}$, then the heat transfer coefficient between these two components, $h_{\sigma \bar{\sigma}}$, can be calculated as

$$
h_{\sigma \bar{\sigma}}=\frac{q}{T_{f}-\overline{T_{p}}}
$$

From the previous section, we have obtained the temperature of the liquid and solid phases of the PCM particle, $T_{1}(x, t)$ and $T_{2}(x, t)$, and the location of the solid-liquid interface $s(t)$, at time $t$. From the energy balance point of view, we have 


$$
q=-k_{2} \frac{\partial T_{2}}{\partial x}=h_{\sigma \bar{\sigma}}\left(T_{f}-\overline{T_{p}}\right)
$$

then the heat transfer coefficient between the PCM particle and the carrier fluid at time $t$ is

$$
h_{\sigma \bar{\sigma}}(t)=\frac{-k_{2} \frac{\partial T_{2}}{\partial x}}{T_{f}-\overline{T_{p}}}=\frac{k_{2} \frac{T_{m}-T_{i}}{s(t)}}{T_{f}-\overline{T_{p}}}=\frac{k_{2}}{s(t)} \frac{T_{m}-T_{i}}{T_{f}-\overline{T_{p}}}
$$

To solve Eq. (5.29), we need to find $\overline{T_{p}}$ first. At time $t$, the mean temperature of the melting PCM can be taken as the average of the liquid and solid phases temperature:

$$
\overline{T_{p}}=\frac{\int_{0}^{s(t)} T_{1}(x, t) \mathrm{d} x+\int_{s(t)}^{x} T_{2}(x, t) \mathrm{d} x}{x}
$$

then by plugging Eq. (5.30) back to Eq. (5.29), we can solve for $h_{\sigma \bar{\sigma}}$ at time $t$.

\section{Challenges in solution method 1}

There are two major challenges in this solution method: first, from our simulation results in the previous chapter, the temperature field of the nanofluid is not so sensitive to the value of $h_{\sigma \bar{\sigma}}$; we might need to propose a new source term in the TLBM model to better incorporate the phase change effect. Second, the derivation for this approach is based on $T_{i}$ and $T_{0}$ being constants, so that the phase interface $s(t)$ can be determined at different time $t$; while in our nanofluid simulation, the temperature of the nanopaticle and the carrier fluid are not constants, they will change at different time steps; more research is needed to figure this out. 


\subsubsection{Solution method 2}

In this section, the formulation of an enthalpy-based LBM model proposed by Chatterjee and Chakraborty for diffusion dominated solid-liquid phase transformation will be provided. The formulation is based on D2Q9 model.

\section{Continuum model}

The equivalent single-phase thermal energy diffusion equation in terms of total enthalpy for a phase change problem can be given in a two-dimensional Cartesian coordinate system as

$$
\frac{\partial}{\partial t}(\rho H)=k\left[\frac{\partial^{2} T}{\partial x^{2}}+\frac{\partial^{2} T}{\partial y^{2}}\right]
$$

The total enthalpy $H$ has two parts, sensible enthalpy and latent enthalpy:

$$
H=c_{p} T+\Delta H
$$

In order to establish a mushy phase change, the latent heat contribution is specified as function of temperature $T$, and the resulting expression is

$$
\Delta H=f(T)= \begin{cases}L & \text { for } T>T_{l} \\ f_{l} L & \text { for } T_{s} \leq T \leq T_{l} \\ 0 & \text { for } T<T_{s}\end{cases}
$$

where $T_{s}$ and $T_{l}$ represent the temperatures at the beginning and end of a phase change, respectively; $\Delta H$ is the latent enthalpy content of a control volume; $L$ is the latent heat of fusion; $f_{l}$ is the liquid phase fraction and is defined as

$$
f_{l}=\frac{\Delta H}{L}
$$

Substitution of the total enthalpy in energy equation yields 


$$
\frac{\partial}{\partial t}\left(\rho c_{p} T\right)=k\left[\frac{\partial^{2} T}{\partial x^{2}}+\frac{\partial^{2} T}{\partial y^{2}}\right]-\frac{\partial}{\partial t}(\rho \Delta H)
$$

where the latent heat appears as a heat source term in the governing equation. If the density $\rho$ and the specific heat $c_{p}$ are explicitly independent of time, and the thermal conductivity $k$ is independent of position, Eq. (5.35) can be written as

$$
\frac{\partial T}{\partial t}=\alpha\left[\frac{\partial^{2} T}{\partial x^{2}}+\frac{\partial^{2} T}{\partial y^{2}}\right]+\Phi
$$

where $\alpha$ is the thermal diffusivity, and $\Phi$ can be regarded as a latent heat source term:

$$
\Phi=-\frac{L}{c_{p}} \frac{\partial f_{l}}{\partial t}
$$

\section{LBM model}

In order to develop a phase change model in a lattice Boltzmann framework, the latent heat source term of Eq. (5.36) needs to be retained in the discretized Boltzmann equation. Thus, the discretized form of the phase change LBM model takes the form:

$$
g_{i}\left(\mathbf{x}+\mathbf{e}_{i} \delta t, t+\delta t\right)=g_{i}(\mathbf{x}, t)-\frac{1}{\tau_{T}}\left[g_{i}(\mathbf{x}, t)-g_{i}^{e q}(\mathbf{x}, t)\right]-\Delta t \Phi_{i} \quad(i=0,1, \ldots, 8)
$$

where $\Phi_{i}$ represents the source which affects the distribution function $g_{i}$ and comes from the direction $i$ in a lattice. $\Phi_{i}$ is taken as

$$
\Phi_{i}=w_{i} \Phi
$$

The other parameters are defined as

$$
\begin{aligned}
& T=\sum_{i=0}^{8} g_{i} \\
& g_{i}^{e q}=w_{i} T
\end{aligned}
$$




$$
\alpha=c_{s}^{2} \delta t\left(\tau_{T}-\frac{1}{2}\right)
$$

As proved by Jiaung et al. (2001) and Chatterjee and Chakraborty (2005), the macroscopic energy diffusion equation Eq. (5.36) can be recovered from Eq. (5.38) by a multi-scale Chapman-Enskog expansion. Therefore, Eq. (5.38) can be used to simulate the solid-liquid phase-change phenomena.

\section{Numerical solution procedure}

The above model can be used to simulate solid-liquid phase change in a pure substance which has discrete interfaces between the respective phases. However, for a multi-component system, there is no sharp interface between solid and liquid phases, in a macroscopic sense. In these situations, a more convenient approach can be a fixed-grid enthalpy-based methodology, in which transport equations for individual phases are volume-averaged to come up with equivalent single-phase conservation equations that are valid over the entire domain, irrespective of the constituent phases locally present. A separate equation for evolution of liquid fraction is solved in conjunction with the above set of conservation equations, which implicitly specify and update the interfacial locations with respect to space and time. To achieve this purpose, dynamic evolution/absorption of latent heat is accounted for by a continuous update of nodal latent enthalpy values of each computational cell, in consistency with the prevailing temperature field. This update is reflected in the energy conservation equation, as either a heat source term or a heat sink.

In a typical simulation, during each marching time step, the iterative procedures for the nonlinear term were executed as follows: 
1). The $n$th iteration value at the new time level $t+\Delta t$ for the particle distribution function is evaluated according to Eq. (5.38) as

$$
\begin{aligned}
g_{i}^{n}\left(\mathbf{x}+\mathbf{e}_{i} \delta t, t+\delta t\right) & =g_{i}(\mathbf{x}, t)-\frac{1}{\tau_{T}}\left[g_{i}(\mathbf{x}, t)-g_{i}^{e q}(\mathbf{x}, t)\right] \\
& -\Delta t w_{i} \frac{L}{c_{p}}\left[\frac{f_{l, i}^{n-1}(t+\Delta t)-f_{l, i}(t)}{\Delta t}\right]
\end{aligned}
$$

2). Temperatures at the $n$th iteration level are then calculated according to Eq. (5.40).

3). The total enthalpies at the $n$th iteration level are then calculated as

$$
\Delta H_{p}^{n}=\Delta H_{p}^{n-1}+\lambda\left[h_{p}^{n-1}-\left(c_{p} T_{m}\right)^{n-1}\right]
$$

where $h_{p}$ is the enthalpy of the concerned cell, $\lambda$ is a suitable relaxation factor to smoothen convergence, and $T_{m}$ is the phase change temperature.

4). The liquid-phase fraction at the current iteration level are then updated according to Eq. (5.34).

5). The obtained value $f_{l}^{n}$ is the used for the next iteration level $n+1$, steps $1-4$ are repeated until the following convergence criterion is satisfied:

$$
\min \left(\left|\frac{T^{n+1}-T^{n}}{T^{n}}\right|,\left|\frac{f_{l}^{n+1}-f_{l}^{n}}{f_{l}^{n}}\right|\right) \leq 10^{-8}
$$

\section{Challenges in solution method 2}

This solution method has been used to simulate phase-change of a pure substance with/without the presence of fluid flow (Chatterjee and Chakraborty, 2005 and 2006). In the PCM particle suspension flow, the PCM particle and the carrier fluid are different 
components, thus, we can not use this approach directly in our research. More research is needed to find how to apply this approach to multicomponent phase-change problems. 


\section{LIST OF REFERENCES}

Ahuja, A.S., 1975', "Augmentation of Heat Transfer in Laminar Flow of Polystyrene Suspensions. I. Experiments and Results", Journal of Applied Physics, Vol. 46, No. 8, pp. 3408-3416.

Ahuja, A.S., 1975², "Augmentation of Heat Transfer in Laminar Flow of Polystyrene Suspensions. II. Analysis of the data", Journal of Applied Physics, Vol. 46, No. 8, pp. 3417-3425.

Arrhenius, S., 1887, Zeitschrift Physikalische Chemie, Vol. 1, p. 287.

Artoli, A.M., Kandhai, D., Hoefsloot, H.C.J., Hoekstra, A.G., and Sloot, P.M.A., 2004, "Lattice BGK simulation of flow in a symmetric bifurcation", Future Generation Computer Systems, Vol. 20, pp. 909-916.

Bahrami, P.A., 1982, "Fusible pellet transport and storage of heat", The $3^{\text {rd }}$ Joint Thermophysics, Fluids, Plasma and Heat Transfer Conference of American Institute of Aeronautics and Astronautics and American Society of Mechanical Engineers, June 7-11, St. Louis, MO.

Bauer, R., and Schlunder, E.U., 1978, "Effective Radial Thermal Conductivity of Packing in Gas Flow," International Chemical Engineering, Vol. 18, pp. 189-204.

Bicerano, J., Douglas, J.F., and Brune, D.A., 1999, "Model for the viscosity of particle dispersions", Journal of macromolecular science, Reviews in macromolecular chemistry and physics, Vol. 39, No. 4, pp. 561-642.

Bouillard, J.X., Lyczkowski, R.W., and Gidaspow, D., 1989, "Porosity distributions in a fluidized bed with an immersed obstacle”, AIChE Journal, Vol. 35, No. 6, pp. 908-922.

Charunyakorn, P., Sengupta, S., and Roy, S.K., 1991, "Forced convection heat transfer in microencapsulated phase change material slurries: flow in circular ducts", International Journal of Heat and Mass Transfer, Vol. 34, No. 3, pp. 819-833.

Chatterjee, D., and Chakraborty, S., 2005, "An enthalpy-based lattice Boltzmann model for diffusion dominated solid-liquid phase transformation", Physics Letters A, Vol. 341, pp. 320-330.

Chatterjee, D., and Chakraborty, S., 2006, "A hybrid lattice Boltzmann model for solidliquid phase transition in presence of fluid flow", Physics Letters A, Vol. 351, pp. 359367. 
Chen, H., Chen, S., and Matthaeus, W.H., 1992, "Recovery of the Navier-Stokes equations using a lattice-gas Boltzmann method", Physical Review A, Vol. 45, No. 8, pp. R5339-R5342.

Chen, H., and Teixeira, C., 2000, H-theorem and origins of instability in thermal lattice Boltzmann models, Computer Physics Communications, Vol. 129, No. 1-3, pp. 21-31.

Chen, K., and Chen, M.M., 1987, "An analytical and experimental investigation of the convective heat transfer of phase change suspension flows", Proceeding of the International Symposium on Multiphase Flow (II), pp. 496-501, Zhejiang University Press, Zhejiang, China.

Chen, S.Y., and Doolen, G.D., 1998, "Lattice Boltzmann method for fluid flows", Annual Review of Fluid Mechanics, Vol. 30, pp. 329-364.

Chen, S., Martínez, D., and Mei, R., 1996, "On boundary conditions in lattice Boltzmann method", Physics of Fluids, Vol. 8, No. 9, pp. 2527-2536.

Choi, E., Cho, Y.I., and Lorsch, H.G., 1994, "Forced convection heat transfer with phasechange-material slurries: turbulent flow in a circular tube", International Journal of Heat and Mass Transfer, Vol. 37, No. 2, pp. 207-215.

Choi, S.U.S., 1995, "Enhancing thermal conductivity of fluids with nanoparticles", Developments and Applications of Non-Newtonian Flows FED-vol.231/MD-vol.66, pp. 99-105.

Chong, J.S., Christiansen, E.B., and Baer, A.D., 1971, "Rheology of concentrated dispersions", Journal of Applied Polymer Science, Vol. 15, pp. 2007-2021.

Cleary, C., Day, S., Lindsay, R., Murry, C., Gupta, R., Larkin, B., Thompson, H., Wiggin, M., and Young, J.C.O'C, 1990 "Hydraulic characteristics of ice slurry and chilled water flows", IEA District Heating: Advanced Energy Transmission Fluid - Final Report of Research, Novem BV, Sittard, Netherlands.

Colvin, D.P., Bryant, Y.B., Mulligan, J.C., and Duncan, J.D., 1989, "Microencapsulated phase change heat transfer systems", WRDC-TR-89-3072, U.S. Air Force Wright R\&D Center, $\mathrm{OH}$.

Colvin, D.P., and Mulligan, J.C., 1990, "Method for using a PCM suspension to enhance heat transfer in liquids", U.S. Patent 4911232.

De Fabritiis, G., Mancini, A., Mansutti, D., and Succi, S., 1998, "Mesoscopic models of liquid/solid phase transitions", International Journal of Modern Physics C, Vol. 9, No. 8, pp. 1405-1415. 
d'Humières, D., Lallemand, P., and Frisch, U., 1986, "Lattice gas model for 3D hydrodynamics", Europhysics Letters, Vol. 2, pp. 291-297.

Drexler, K.E., 1992, "Nanosystems, Molecular Machinery, Manufacturing and Computation", John Wiley \& Sons, New York.

Dupuis, A., 2002, "From a lattice Boltzmann model to a parallel and reusable implementation of a virtual river", Ph.D. dissertation, Université de Genève.

Eastman, J.A., Phillpot, S.R., Choi, S.U.S., and Keblinski, P., 2004, “Thermal transport in nanofluids", Annual Review of Materials Research, Vol. 34, pp. 219-246.

Eilers, H., 1941, Kolloid Zeitschrif, Vol. 97, p. 313.

Einstein, A., 1906, "Eine neue Bestimmung der Moleküldimensionen", Annalen der Physik, Vol. 19, pp. 289-306.

Einstein, A., 1911, "Berichtigung zu meiner Arbeit: Eine neue Bestimmung der Moleküldimensionen”, Annalen der Physik, Vol. 34, pp. 591-592.

Faghri, A., and Zhang, Y.W., 2006, "Transport phenomena in multiphase systems", Elsevier Inc., USA.

Frank, M.P., and Knight, F.T., Jr., 1998, "Ultimate theoretical models of nanocomputers", Nanotechnology, Vol. 9, No. 3, pp. 162-176.

Frankel, N.A., and Acrivos, A., 1967, "On the viscosity of a concentrated suspension of solid spheres”, Chemical Engineering Science, Vol. 22, No. 6, pp. 847-853.

Frisch, U., d'Humières, D., Hasslacher, B., Lallemand, P., Pomeau, Y., and Rivet, J. P., 1987, "Lattice gas hydrodynamics in two and three dimensions", Complex Systems, Vol. 1, pp. 649-707.

Frisch, U., Hasslacher, B., and Pomeau, Y., 1986, "Lattice-gas automata for the NavierStokes equation”, Physical Review Letters, Vol. 56, pp. 1505-1508.

Ginzbourg, I., and d'Humières, D., 1995, "Local second-order boundary methods for lattice Boltzmann models”, Journal of Statistical Physics, Vol. 84, No. 5-6, pp. 927-971.

Gidaspow, D., 1986, "Hydrodynamics of Fluidization and Heat Transfer: Supercomputer Modeling," Applied Mechanics Reviews, Vol. 39, No. 1, pp. 1-23.

Goel, M., Roy, S.K., and Sengupta, S., 1994, "Laminar Forced Convection Heat Transfer in Microcapsulated Phase Change Material Suspensions", International Journal of Heat and Mass Transfer, Vol. 37, No. 4, pp. 593-604. 
Graham, A.L., 1981, "On the viscosity of suspensions of solid spheres", Applied Scientific Research, Vol. 37, pp. 275-286.

Gromoll, B., 1994, “Advanced Micro Air-Cooling Systems for High Density Packaging”, The $10^{\text {th }}$ IEEE Semiconductor Thermal Measurement and Management Symposium, February 1-3, pp. 53-58.

Gunstensen, A.K., Rothman, D.H., Zaleski, S., and Zanetti, G., 1991, "Lattice Boltzmann model of immiscible fluids", Physical Review A, Vol. 43, No. 8, pp. 4320-4327.

Guth, E., and Simha, R., 1936, Kolloid Zeitschrift, Vol. 74, p. 266.

Hao, Y.L., and Tao, Y.-X., 2000, "Three-dimensional numerical simulation of liquidsolid flow with phase change using SIMPLE method", The 2000 ASME Fluid Engineering Division Summer Meeting, June 11-15, Boston, MA.

Hao, Y.L., and Tao, Y.-X., 2004, "A Numerical Model For Phase Change Suspension Flow In Microchannels", Numerical Heat Transfer: Part A., Vol. 46, No. 1, pp. 55-77.

Hardy, J., de Pazzis, O., and Pomeau, Y., 1976, "Molecular dynamics of a classical lattice gas: transport properties and time correlation functions", Physical Review A, Vol. 13, No. 5, pp. 1949-1961.

Hart, R., and Thornton, F., 1982, "Microencapsulation of phase change materials", Final Report Contract No. 82-80, Ohio Department of Energy, Ohio.

He, D., and Ekere, N.N., 2001, "Viscosity of concentrated noncolloidal bidisperse suspensions", Rheologica Acta, Vol. 40, pp. 591-598.

He, X.Y., Chen, S.Y., and Doolen, G.D., 1998, "A novel thermal model for the lattice Boltzmann method in incompressible limit", Journal of Computational Physics, Vol. 146, pp. 282-300.

He, X., Chen, S., and Zhang, R., 1999, “A lattice Boltzmann scheme for incompressible multiphase flow and its application in simulation of Rayleigh-Taylor instability", Journal of Computational Physics, Vol. 152, No. 2, pp. 642-663.

He, X., and Luo, L.S., 1997, "Theory of the lattice Boltzmann method: From the Boltzmann equation to the lattice Boltzmann equation", Physical Review E, Vol. 56, No. 6, pp. 6811-6817.

Higginbotham, G.H., Oliver, D.R., and Ward, S.G., 1958, "Studies of the viscosity and sedimentation of suspensions. Part 4. Capillary-tube viscometry applied to stable suspensions of spherical particles", British Journal of Applied Physics, Vol. 9, pp. 372377. 
Higuera, F.J., and Jiménez, J., 1989, "Boltzmann approach to lattice gas simulations", Europhysics Letters, Vol. 9, pp. 663-668.

Higuera, F.J., Succi, S., and Benzi, R., 1989, "Lattice gas dynamics with enhanced collisions", Europhysics Letters, Vol. 9, pp. 345-349.

Hu, X.X., and Zhang, Y.P., 2002, "Novel insight and numerical analysis of convective heat transfer enhancement with microencapsulated phase change material slurries: laminar flow in a circular tube with constant heat flux", International Journal of Heat and Mass Transfer, Vol. 45, pp. 3163-3172.

Huang, H.B., 2007, "Axisymmetric and three-dimensional lattice Boltzmann models and their applications in fluid flows", Ph.D. dissertation, National University of Singapore.

Ilic, V., and Phan-Thien, N., 1994, "Viscosity of concentrated suspensions of spheres", Rheologica Acta, Vol. 33, pp. 283-291.

Inaba, H., Kim, M.J., and Horibe, A., 2004, "Melting heat transfer characteristics of microencapsulated phase change material slurries with plural microencapsules having different diameters", Journal of Heat Transfer, Vol. 126, pp. 558-565.

Inamuro, T., Ogata, S., Tajima, S., and Konishi, N., 2004, "A lattice Boltzmann method for incompressible two-phase flows with large density differences", Journal of Computational Physics, Vol. 198, pp. 628-644.

Jackson, R., 1971, "Fluid mechanical theory," In: J. F. Davidson, and J. Harrison (eds.), Fluidization. Academic, London, pp. 63-119

Jiaung, W.S., Ho, J.R., and Kuo, C.P., 2001, "Lattice Boltzmann method for the heat conduction problems with phase change", Numerical Heat Transfer, Part B, Vol. 39, pp. 167-187.

Kasza, K.E., and Chen, M.M., 1982, "Development of enhanced heat transfer/transport/storage slurries for thermal-system improvement", $A N L-82-50$, Argonne National Lab., Illinois.

Khanafer, K., Vafai, K., and Lightstone, M., 2003, "Buoyancy-driven heat transfer enhancement in a two-dimensional enclosure utilizing nanofluids", International Journal of Heat and Mass Transfer, Vol. 46, No. 19, pp. 3639-3653.

Kitano, T., Kataoka, T., and Shirota, T., 1981, "An empirical equation of the relative viscosity of polymer melts filled with various inorganic fillers", Rheologica Acta, Vol. 20, No. 2, pp. 207-209. 
Kovar, J., and Fortelny, I., 1984, "Effect of polydispersity on the viscosity of a suspension of hard spheres", Rheologica Acta, Vol. 23, pp. 454-456.

Krieger, I.M., and Dougherty, T.J., 1959, “A mechanism for non-Newtonian flow in suspensions of spherical particles", Transactions of the Society of Rheology, Vol. 3, No. 1, pp. 137-152.

Krieger, I.M., 1972, "Rheology of monodisperse latices", Advances in Colloid and Interface Science, Vol. 2, No. 3, pp. 111-136.

Leland, J., and Recktenwald, G., 2003, "Optimization of a phase change heat sink for extreme environments", Proceedings of the $19^{\text {th }}$ Annual IEEE Semiconductor Thermal Measurement and Management Symposium, March 11-13, San Jose, CA.

Li, A., and Ahmadi, G., 1992, "Dispersion and deposition of spherical particles from point sources in a turbulent channel flow", Aerosol Science and Technology, Vol. 16, pp. 209-226.

Maier, R.S., Bernard, R.S., and Grunau, D.W., 1996, "Boundary conditions for the lattice Boltzmann method", Physics of Fluids, Vol. 8, No. 7, pp. 1788-1801.

Maloji, P., and Y.-X. Tao, 2006, "Optimal ratio of heat removal rate to pumping power for PCM emulsion fluids: Low Reynolds number limits”, 2006 ASME International Mechanical Engineering Congress and Exposition, November 5-10, Chicago, Illinois, USA.

McMahon, W.A., Jr., Harlowe, W.W., Jr., and Mangold, D.I., 1982, "Feasibility study of utilizing phase change coolant for protective garment", Final Report Contract No. DAAK60-81-C-0098, U.S. Army Natick Research and Development Command, MA.

McNamara, G.R., and Zanetti, G., 1988, "Use of the Boltzmann equation to simulate lattice-gas automata”, Physical Review Letters, Vol. 61, No. 14, pp.2332-2335.

Mechalick, E.M., and Tweedie, A.T., 1975, “Two component thermal energy storage material", Report NSF/RANN/SE/AER-74-09186, National Science Foundation, Washington, D.C.

Metzner, A.B., 1985, "Rheology of suspensions in polymeric liquids", Journal of Rheology, Vol. 29, No. 6, pp. 739-775.

Miller, W., Succi, S., and Mansutti, D., 2001, "Lattice Boltzmann model for anisotopic liquid-solid phase transition”, Physical Review Letters, Vol. 86, No. 16, pp. 3578-3581.

Mooney, M., 1951, "The viscosity of a concentrated suspension of spherical particles", Journal of Colloid Science, Vol. 6, No. 2, pp. 162-170. 
O'Conner, J.P., and Weber, R.M., 1997, “Thermal management of electronic packages using solid-to-liquid phase change techniques", International Journal of Microcircuits and Electronic Packaging, Vol. 20, pp. 593-601.

Ortega, A., 2002, "What Are the Heat Flux Limits of Air Cooling?", 2002 International Mechanical Congress and Exposition, Panel: Challenges in Cooling High Heat Flux Electronics Systems, New Orleans, LA, November 17-22.

Pal, D., and Joshi, Y.K., 1998, "Thermal management of an avionics module using solidliquid phase-change materials", Journal of Thermophysics and Heat Transfer, Vol. 12, No. 2, pp. 256-262.

Qian, Y.H., d'Humières, D., and Lallemand, P., 1992, "Lattice BGK models for NavierStokes equation", Europhysics Letters, Vol. 17, pp. 479-484.

Robinson, J., 1949, "The viscosity of suspensions of spheres", Journal of Physical Chemistry, Vol. 53, No. 7, pp. 1042-1056.

Roscoe, R., 1952, "The viscosity of suspensions of rigid spheres", British Journal of Applied Physics, Vol. 3, pp. 267-269.

Rowe, P.N., 1961, "Drag forces in a hydraulic model of a fluidized bed", Transactions of the Institution of Chemical Engineers, Vol. 39, pp. 175-180.

Russel, W.B., Saville, D.A., and Schowalter W.R., 1989, "Colloidal dispersions", Cambridge University Press, Cambridge, England.

Rutgers, I.R., 1962, "Relative Viscosity of Suspensions of Rigid Spheres in Newtonian Liquids", Rheologica Acta, Vol. 2, No. 3, pp. 202-210.

Sengun, M.Z., and Probstein, R.F., 1989, "Bimodal model of slurry viscosity with application to coal-slurries. Part 2. High shear limit behavior", Rheologica Acta, Vol. 28, No. 5, pp. 394-401.

Shan, X.W., 1997, "Simulation of Rayleigh-Bénard convection using a lattice Boltzmann method", Physical Review E, Vol. 55, No. 3, pp. 2780-2788.

Shan, X., and Chen, H., 1993, "Lattice Boltzmann model for simulating flows with multiple phases and components", Physical Review E, Vol. 47, pp. 1815-1819.

Shan, X., and Chen, H., 1994, "Simulation of nonideal gases and liquid-gas phase transitions by the lattice Boltzmann equation", Physical Review E, Vol. 49, No. 4, pp. 2941-2948. 
Shan, X., and Doolen, G., 1995, "Multicomponent lattice-Boltzmann model with interparticle interaction”, Journal of Statistical Physics, Vol. 81, pp. 379-393.

Shan, X., and Doolen, G., 1996, "Diffusion in a multicomponent lattice Boltzmann equation model”, Physical Review E, Vol. 54, pp. 3614-3620.

Shu, C., Peng, Y., and Chew, Y.T., 2002, "Simulations of natural convection in a square cavity by Taylor series expansion- and least squares- based lattice Boltzmann method", International Journal of Modern Physics, Vol. 13, No. 10, pp. 1399-1414.

Storms, R.F., Ramarao, B.V., and Weiland, R.H., 1990, "Low shear rate viscosity of bimodally dispersed suspensions", Powder Technology, Vol. 63, No. 3, pp. 247-259.

Strerling, J.D., and Chen, S., 1996, "Stability analysis of lattice Boltzmann methods", Journal of Computational Physics, Vol. 123, No. 1, pp. 196-206.

Succi, S., 2001, "The lattice Boltzmann equation: from fluid dynamics and beyond", Oxford University Press, New York.

Swift, M.R., Orlandini, E., Osborn, W.R., and Yeomans J.M., 1996, "Lattice Boltzmann simulations of liquid-gas and binary fluid systems", Physical Review E, Vol. 54, No. 5, pp. 5041-5052.

Tao, Y.-X., Moreno, R., and Hao, Y.L., 2003, "Design analysis of a 3-D, ultra-high performance, scalable, micro convective heat sink with NPCM", First International Conference on Microchannels and Minichannels, April 24-25, Rochester, New York.

Tao, Y.X., Xing, K.Q., Maloji, P., and Hao, Y.L., 2007, “Low Reynolds number limit of heat transfer enhancement with phase change material slurry flow in micro/mini channels", Journal of Enhanced Heat Transfer, Vol. 14, No. 2, in press.

Thomas, D.G., 1965, "Transport characteristics of suspension: VIII. A note on the viscosity of Newtonian suspensions of uniform spherical particles", Journal of colloid and interface science, Vol. 20, No. 3, pp. 267-277.

Tuckerman, D.B., and Pease, R.F.W., 1981, "High-performance heat sinking for VLSI", IEEE Electron Device Letters, Vol. 2, No. 5, pp. 126-129.

Vand, V., 1945, “Theory of Viscosity of Concentrated Suspensions", Nature, Vol. 155, pp. 364-365.

Vand, V., 19481, "Viscosity of solutions and suspensions. I. Theory", Journal of Physical Chemistry, Vol. 52, No. 2, pp. 277-299. 
Vand, V., 1948², "Viscosity of solutions and suspensions. II. Experimental determination of the viscosity-concentration function of spherical suspensions", Journal of Physical Chemistry, Vol. 52, No. 2, pp. 300-314.

Vassallo, P., Kumar, R., and d'Amico, S., 2004, "Pool boiling heat transfer experiments in silica-water nanofluids", International Journal of Heat and Mass Transfer, Vol. 47, No. 2, pp. 407-411.

Wakao, N., and Kagei, S., 1982, "Heat and Mass Transfer in Packed Beds", Gordon and Breach Science Publishers, New York.

Wang, X.W., Xu, X.F., and Choi, S.U.S., 1999, "Thermal conductivity of nanoparticlefluid mixture", Journal of Thermophysics and Heat Transfer, Vol. 13, No. 4, pp. 474-480.

Wang, B.X., Zhou, L.P., and Peng, X.F., 2003, "A fractal model for predicting the effective thermal conductivity of liquid with suspension of nanoparticles", International Journal of Heat and Mass Transfer, Vol. 46, pp. 2665-2672.

Xuan, Y., and Roetzel, W., 2000, "Conception for heat transfer correlation of nanofluids", International Journal of Heat and Mass Transfer, Vol. 43, No. 9, pp. 3701-3707.

Xuan, Y., and Yao, Z., 2005, "Lattice Boltzmann model for nanofluids", Heat and Mass Transfer, Vol. 41, No. 3, pp. 199-205.

Yamagishi, Y., Takeuchi, H., Pyatenko, A.T., and Kayukawa, N., 1999, "Characteristics of microencapsulated PCM slurry as a heat transfer fluid", AIChE Journal, Vol. 45, No. 4, pp. 696-707.

Yuan, P., 2005, “Thermal lattice Boltzmann two-phase flow model for fluid dynamics", Ph.D. dissertation, University of Pittsburgh.

Zhang, Y.W., and Faghri, A., 1995, "Analysis of forced convection heat transfer in microencapsulated phase change material suspensions", Journal of Thermophysics and Heat Transfer, Vol. 9, No. 4, pp. 727-732.

Zheng, N., and Wirtz, R.A., 2000, "Methodology for designing a hybrid thermal energy storage heat sink", Proceedings of the 2000 ASME International Mechanical Engineering Congress and Exposition, November 5-10, Orlando, Florida.

Ziegler, D.P., 1993, "Boundary conditions for lattice Boltzmann simulations", Journal of Statistical Physics, Vol. 71, No. 5-6, pp. 1171-1177.

Zou, Q., and He, X., 1997, "On pressure and velocity boundary conditions for the lattice Boltzmann BGK model”, Physics of Fluids, Vol. 9, No. 6, pp. 1591-1598. 
VITA

\section{KEQIANG XING}

EDUCATION

1998

2000

2005
B.S. Thermal Engineering

Chongqing University

Chongqing, China

M.S. Engineering Thermophysics

Chongqing University

Chongqing, China

Doctoral Candidate in Mechanical Engineering Florida International University

Miami, Florida, USA

\section{ACTIVITIES/HONORS AND AWARDS}

American Society of Mechanical Engineers (ASME) (Member since 2004)

American Society of Heating Refrigeration and Air Conditioning Engineers (ASHRAE) (Member since 2007)

Department of Energy's 2005 Solar Decathlon FIU Team Member

Certificate of Appreciation from ASME 2007 Early Career Technical Conference for Assistance as Invited Reviewer of the Technical Journal

\section{LIST OF JOURNAL PUBLICATIONS}

K.Q. Xing, Y.-X. Tao, and Y.L. Hao, 2005, "Performance evaluation of liquid flow with PCM particles in microchannels", Journal of Heat Transfer, Vol. 127, No. 8, pp. 931-940.

Y.-X. Tao, K.Q. Xing, P. Maloji, and Y.L. Hao, 2007, "Low Reynolds number limit of heat transfer enhancement with phase change material slurry flow in micro/mini channels", Journal of Enhanced Heat Transfer, Vol. 14, No. 2, pp. $105-122$. 


\section{LIST OF CONFERENCE PAPERS}

K.Q. Xing, Y.-X. Tao, and Y.L. Hao, 2004, "Performance evaluation of liquid flow with NPCM particles in microchannels", 2004 ASME Heat Transfer/Fluids Engineering Summer Conference, July 11-15, Charlotte, North Carlonia, USA.

K.Q. Xing and Y.-X. Tao, 2005, "Lattice Boltzmann simulation of flow and heat transfer characteristics in a symmetric bifurcation”, 2005 ASME Summer Heat Transfer Conference, July 17-22, San Francisco, California, USA.

Y.-X. Tao, K.Q. Xing, P. Maloji, and Y.L. Hao, 2006, "Low Reynolds number limit of heat transfer enhancement for phase change materials slurry flow in micro/mini channels", $13^{\text {th }}$ International Heat Transfer Conference, August 13-18, Sydney, Australia.

K.Q. Xing, Y.-X. Tao, and Y.L. Hao, 2006, "Slurry viscosity study and its influence on heat transfer enhancement effect of PCM slurry flow in micro/mini channels", 2006 ASME International Mechanical Engineering Congress and Exposition, November 5-10, Chicago, Illinois, USA. 